\title{
Critical Behavior and Crossover Effects in the Properties of Binary and Ternary Mixtures and Verification of the Dynamic Scaling Conception
}

\author{
Dissertation \\ zur Erlangung des Doktorgrades \\ der Mathematisch-Naturwissenschaftlichen Fakultäten \\ der Georg-August-Universität zu Göttingen
}

vorgelegt von

Dipl. Phys. Ireneusz Iwanowski

aus Sorau (Żary)

Göttingen 2007 
D7

Referent: Prof. Dr. Werner Lauterborn

Korreferent: Prof. Dr. Christoph Schmidt

Tag der mündlichen Prüfung: 29.11.07 
...one of the strongest motives that lead men to art and science is escape from everyday life with its painful crudity and hopeless dreariness, from the fetters of one's own ever-shifting desires. A finely tempered nature longs to escape from the personal life into the world of objective perception and thought.

ALBERT EINSTEIN

(1879-1955)

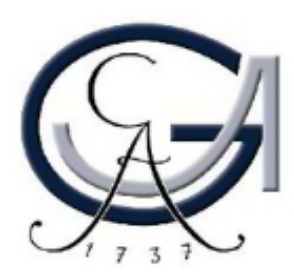

Dla rodziców Anny i Kazimierza Iwanowskich 



\section{Contents}

1 Introduction 1

2 Thermodynamics of Critical Phenomena 5

2.1 Phase transitions . . . . . . . . . . . . . . . . . . 5

2.2 Critical fluctuations . . . . . . . . . . . . . . . . 7

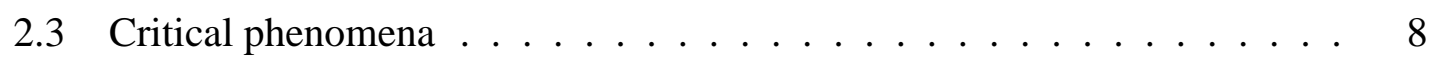

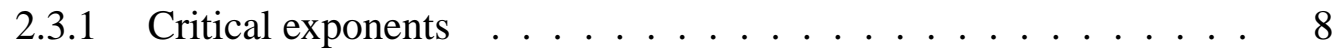

2.3.2 Static scaling hypothesis . . . . . . . . . . . . . 9

2.3.3 Dynamic scaling hypothesis and critical slowing down . . . . . 10

2.3.4 Renormalization of critical exponents . . . . . . . . . . . 12

2.3.5 Critical opalescence and equal volume criterions _ . . . . . . 12

2.4 Phase diagrams . . . . . . . . . . . . . . . . . . . . 12

2.4.1 Phase diagrams for binary liquids at constant pressure . . . . . 12

2.4.2 Phase diagrams of ternary mixtures at constant pressure $\ldots \ldots 13$

3 Experimental Methods $\quad 19$

3.1 General aspects . . . . . . . . . . . . . . . . . . . . . . 19

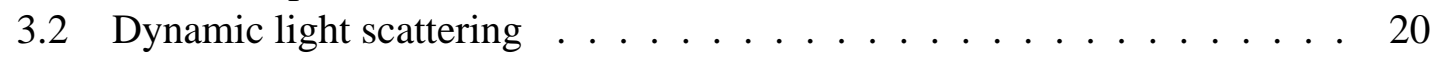

3.2 .1 Electromagnetic scattering theory $\ldots \ldots \ldots \ldots \ldots$

3.2.2 Spectrum of scattered field - hydrodynamic considerations . . . . 22

3.2.3 Data evaluation of dynamic light scattering . . . . . . . . 25

3.2 .4 Self-beating spectroscopy $\ldots \ldots \ldots \ldots \ldots$

3.2 .5 Technical equipment . . . . . . . . . . . . . . . . 27

3.3 Ultrasonic techniques . . . . . . . . . . . . . . . . . 28

3.3.1 Classical absorption and background contribution . . . . . . . 29

3.3.1.1 Noncritical ultrasonic excess absorption . . . . . . . 30

3.3.1.2 Critical systems and total attenuation spectrum . . . . 33 
3.3.2 Ultrasonic instruments . . . . . . . . . . . . . . . . . 33

3.3.3 Resonator cells $80 \mathrm{kHz}-20 \mathrm{MHz}$.............. 34

3.3.4 Pulse-modulated traveling wave methods . . . . . . . . . . . . 41

3.4 Complementary measurement techniques . . . . . . . . . . . . 46

4 Critical Contribution, Dynamic Scaling and Crossover Theory 51

4.1 Bhattacharjee-Ferrell scaling hypothesis - binary systems . . . . . . . . . 51

4.2 Critical sound attenuation . . . . . . . . . . . . . . 51

4.3 The scaling function $F_{x}(\Omega) \ldots \ldots \ldots \ldots \ldots$

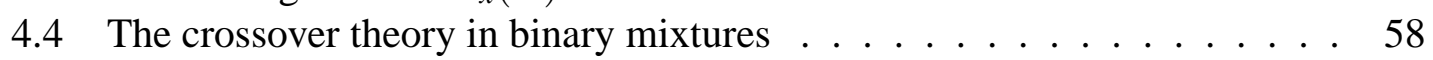

4.5 The crossover theory in ternary mixtures . . . . . . . . . . . 61

5 Experimental Verification of Dynamic Scaling Theories and Discussions 63

5.1 Strategies of verifying the scaling function . . . . . . . . . . 63

5.2 Preparation of critical mixtures . . . . . . . . . . . . . . 66

5.3 Binary systems without and with one additional noncritical relaxation term 67

5.3.1 Phase diagram and critical temperature. . . . . . . . . . . 68

5.3.2 Dynamic light scattering, shear viscosity $\eta_{s}$ and characteristic re-

laxation rate $\Gamma_{0} \ldots \ldots \ldots \ldots$. . . . . . . . . . . . . 69

5.4 Fluctuation correlation length . . . . . . . . . . . . . . . . . . 74

5.5 Ultrasonic spectrometry . . . . . . . . . . . . . . . . 76

5.5.1 Scaling functions . . . . . . . . . . . . . . . . 79

5.5.2 Amplitude $S_{B F}$ and coupling constant $g \ldots \ldots$. . . . . 86

5.6 Systems with complex background contributions _ . . . . . . . . . 88

5.6.1 Isobutoxyethanol-water . . . . . . . . . . . . . . . . . 89

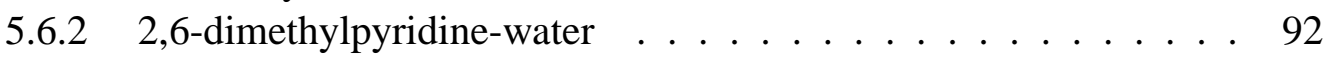

5.6 .3 Triethylamine-water . . . . . . . . . . . . . . . 96

5.7 Ternary system nitroethane-3-methylpentane-cyclohexane . . . . . . . 104

5.7.1 Crossover studies of viscosity and light scattering . . . . . . . . . 107

5.7.2 Dynamic scaling function of a ternary mixture . . . . . . . . 111

5.8 Summarized parameters and surface tension in critical mixtures . . . . 118

6 Conclusions and Outlook 125

Literature I 


\section{Introduction}

Nature comprises a multitude of critical phenomena. Spontaneous symmetry breaking at the origin of the universe and gravitation collapse [1], [2] are spectacular examples. Critical phenomena occur at phase transitions. Theories of phase transitions use methods of catastrophe theory and also of theory of percolation which currently attract considerable attention.

In order to understand critical phenomena, investigations of liquid-liquid phase transitions in binary and ternary mixtures are very instructive. Especially the understanding of the phase behavior and the critical phenomena in ternary mixtures, biophysics and membrane physics have attracted attention during the last years [3], [4], [5]. The essential and most amazing feature of critical phenomena was the discovery of critical point universality indicating that the microscopic structure of fluids becomes unimportant in the vicinity of the critical point. The understanding of such phenomena is also of great importance for chemistry and chemical engineering in procedures like liquid and solid extraction, drying, absorption, distillation and many other chemical reaction processes, as well as for biology in operations like fermentation, biological filtration and syntheses. Moreover, theories of critical phenomena are substantial for many innovative applications such as supercritical extraction, enhanced oil recovery and supercritical pollution oxidation. The importance of the understanding and application of critical phenomena is demonstrated by the recently (08.28.07) provided studies ${ }^{1}$ performed in the International Space Station (ISS). The focus of those investigations were the critical phenomena, in particular the critical slowing down of the phase separation near the critical point of binary colloid-polymer mixtures in a micro gravity environment. The aim of such investigations was to develop fundamental physical concepts previously which had so far been masked by gravity effects.

A first qualitative description of the critical behavior of some special systems was already given at the beginning of last century. Examples are liquid/gas transition and the ferro/paramagnetism transition [6]. An essential step towards a deeper understanding of critical behavior was made by Landau's general theory of phase transitions [7]. Later the theory has been further developed by Onsager, which found the exact solution [8] for

\footnotetext{
${ }^{1}$ National Aeronautics and Space Administration (NASA) Expeditions Assigned. Previous studies on critical systems in micro gravity environment have been performed on in 1997 and 1998 on MIR, Source: http://www.nasa.gov.
} 
the thermodynamic properties of a two-dimensional Ising model, that had been frequently discussed. It was a great surprise to find the theory of Landau to fail completely in predicting the behavior close to the critical point. Fisher, played a leading role by his analysis of experimental data, combining theoretical analysis and numerical calculations, [9], [10], [11]. Important theoretical contributions have been made by Widom [12], Patashinskii and Pokrovski [13], and most important by Kadanoff, [14]. Kadanoff put forward a very important new and original idea which seemed to have a strong influence on the later development of the field. However, his theory did not allow to calculate the critical behavior. The problem was solved in a comprehensive and profound way by Wilson [15]. Wilson was the first to realize that critical phenomena are different from most other phenomena in physics in that close to the critical point one has to deal with fluctuations over widely different scales of length. Nevertheless, investigations which have been performed on critical system have indicated the description of their behavior is not sufficient for a wide temperature range. The range between the background and mean-field behavior could not be described satisfactorily by classic theories of critical phenomena. Consequently, a new formalism has been developed by Albright [68], Burstyn, Sengers, Bhattacharjee and Ferrell, [16], [17], to describe the range between the consolute point and background behavior. This formalism is named crossover theory.

The ideas of universality appear to be also applicable to phase transitions in complex fluids like polymers and polymer solutions, micro-emulsions and liquid crystals, fluids in porous media as well as phospholipid bilayer vesicle solutions. Besides dynamic light scattering an important measurement method to study the cooperative effects in complex fluids is the broad band ultrasonic spectroscopy. Being a non-destructive technique it allows for the measurement of chemical relaxations as well as critical fluctuations. During the past decades various theories have been developed to treat the critical slowing down of the critical ultrasonic attenuation in binary liquids within the framework of the dynamic scaling theory. The most prominent examples of the dynamic scaling theory have been presented by Bhattacharjee and Ferrell [18], [19], [20], [21], [22]. Folk and Moser [23], [24] developed a renormalization group theory of the mode-coupling model and Onuki [25], [26] derived a model from an intuitive description of the bulk viscosity near the critical point. The verification, with the aid of dynamic light scattering and shear viscosity measurements as well as with broad band ultrasonic measurements, of the validity of these dynamic scaling functions of various critical mixtures was one of the aims of the present thesis. Essential quantities are the characteristic relaxation time $\Gamma$, the fluctuations correlation length $\xi$, the mutual diffusion coefficient $D$, as well as the reduced half-attenuation frequency $\Omega_{1 / 2}$, the adiabatic coupling constant $g$, and the critical amplitude $S$ of the ultrasonic attenuation spectra. Also considered was the coupling between the critical contributions and the noncritical relaxations due to chemical processes, as well as the predictions by Procaccia et al. [27], on the critical slowing of those chemical re- 
actions which might occur when a system is approaching its critical demixing point. An additional emphasis of studies of binary mixtures was the verification of the before mentioned crossover theory, with cut-off wave numbers $q_{c}$ and $q_{D}$ as substantial parameters. Investigations on ternary mixtures with different compositions along the plait line were performed to determine the validity of the dynamic scaling hypothesis, especially the dependence of critical quantities on the concentration of the additional third component. With the ternary mixtures special attention has been paid to the crossover behavior, which had been first considered for binary systems. 



\section{Thermodynamics of Critical Phenomena}

In this Chapter the theoretical background of the thermodynamics of critical phenomena in binary and ternary liquid systems, phase transitions as well as the static and dynamic scaling hypotheses are presented. Furthermore, critical exponents and their important role for the description of second order phase transitions are shown. Moreover, typical binary and ternary phase diagrams are presented.

\subsection{Phase transitions}

The first attempt to classify phase transitions was done by Ehrenfest (1933). The Ehrenfest classification scheme was based on grouping of phase transitions into their degree of non-analyticity. Phase transitions were labeled by the lowest derivative of the Gibbs free energy $G(T, p)$ that is discontinuous at the phase transition. Taking a closer look at the increasing degree of derivation, the differences between the phases on consideration get smaller. In fact, it is necessary to raise the question about the sense of differing between phases in this way. It is more practicable to consider derivatives of lower degree only. This leads to the description of so-called first-order phase transitions or discontinuous phase transitions. Characteristic of first-order phase transitions is the discontinuity of the first derivation of the Gibbs free energy $G(T, p)$. Within the scope of critical phenomena in liquids, another kind of phase transitions plays an important role, the so-called second-order phase transi-

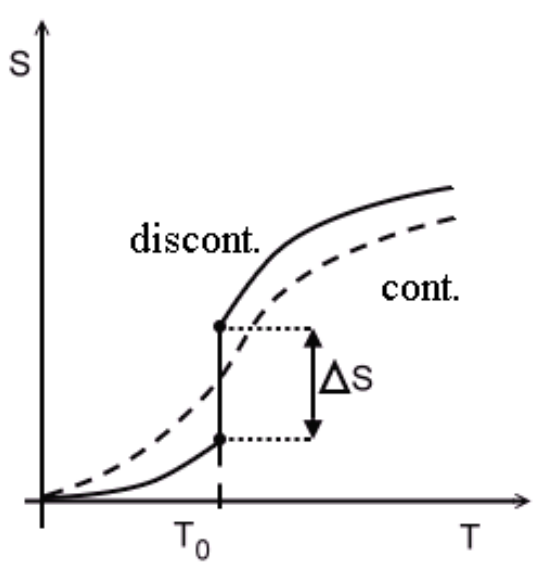

Figure 2.1:

Discontinuous and continuous shape of the entropy of phase transitions: discont. $\Delta S \neq 0 \Leftrightarrow \Delta Q=T_{0} \Delta S$ cont. $S$ is a continuous function of $T \Rightarrow \Delta Q=0$ tions or the continuous phase transitions (see Fig.(2.1)). In this case, the first derivative of Gibbs free energy is continuous and especially in liquid systems the conception of latent heat does no longer exist. Another characteristic parameter of continuous phase 
transitions is the so-called order parameter. An order parameter is a macroscopic quantity which describes the degree of order, or vice versa, the degree of disorder of the thermodynamic system. In a many-particle system two opposite tendencies concur against each other. This behavior can be found in the free energy $F=U-T S$. Here $U$ denotes the inner energy, and $S$ the entropy. In thermodynamic equilibrium, the free energy $F$ has to reach a minimum. This can be realized with smaller inner energy $U$ (high order of a system) or higher entropy $S$ (smaller order of a system). Consequently, the temperature $T$ is the decisive parameter. Liquid-liquid phase transitions, transitions from homogenous state into a heterogonous state of a liquid, lead to a symmetry breakage of the system. In binary as well as ternary liquid systems the difference in the mole fraction of a constituent in the different phases $\Delta x=x^{\prime}-x^{\prime \prime}$ represents an order parameter. An important role in the treatment and classification of phase transitions plays the criterion of stability. According to the second law of thermodynamics, the free energy $F$ is a stability parameter of a mixture. One can make a distinction between three different kinds of stability [28], the thermal stability $\left(\partial^{2} F / \partial T^{2}\right)_{V}<0$, the mechanic stability $\left(\partial^{2} F / \partial V^{2}\right)_{T}<0$ and the dynamic stability $\left(\partial^{2} F / \partial x^{2}\right)_{V, T}>0$. In Fig.(2.2) the criterion of dynamic stability is illustrated, with the aid of free energy of mixing for ideal solution $F^{m}(x)=N_{A} k_{B} T[(1-x) \ln (1-x)+x \ln (x)]$, where $N_{A}$ is the Avogadro constant and $k_{B}$ Boltzmann's constant. The region between points $B$ and $C$ in Fig.(2.2) represents the unstable range of the isobar-isotherm. Outside this area, the criterion of stability is fulfilled. The points $A$ and $D$, which are resulting from the construction of the double-tangent line, represents the equilibrium of the coexisting phases at the mole fractions of $x^{\prime}$ and $x^{\prime \prime}$, respectively. This area corresponds with the so-called binodal of the phase diagram of a binary system. The areas between $A$ and $B$ as well as $C$ and $D$ are representing the meta-stable range, corresponding with the area between the so-called spinodal and the binodal. At the spinodal an interesting phenomenon occurs at phase transitions. Other than at a binodal, in the meta-stable range the solution separates spontaneously into two phases, starting with small fluctuations and proceeding with a decrease in the Gibbs free energy $G, G=H-T S$ (here is $H$ the enthalpy), without a nucleation barrier. Considering now the Gibbs free energy at constant temperature and constant pressure, the following distinctions can be done:

$$
\begin{aligned}
& \left(\partial^{2} G / \partial x^{2}\right)_{T, p}>0 \text { stable and meta-stable } \\
& \left(\partial^{2} G / \partial x^{2}\right)_{T, p}<0 \text { unstable } \\
& \left(\partial^{2} G / \partial x^{2}\right)_{T, p}=0 \text { spinodal. }
\end{aligned}
$$

Changing of composition of the binary system or changing the temperature $T$, leads to the merge of points $B$ and $C$ as well $A$ and $D$. At this so-called critical point the criterion of stability as well the criterion of equilibrium is fulfilled. 


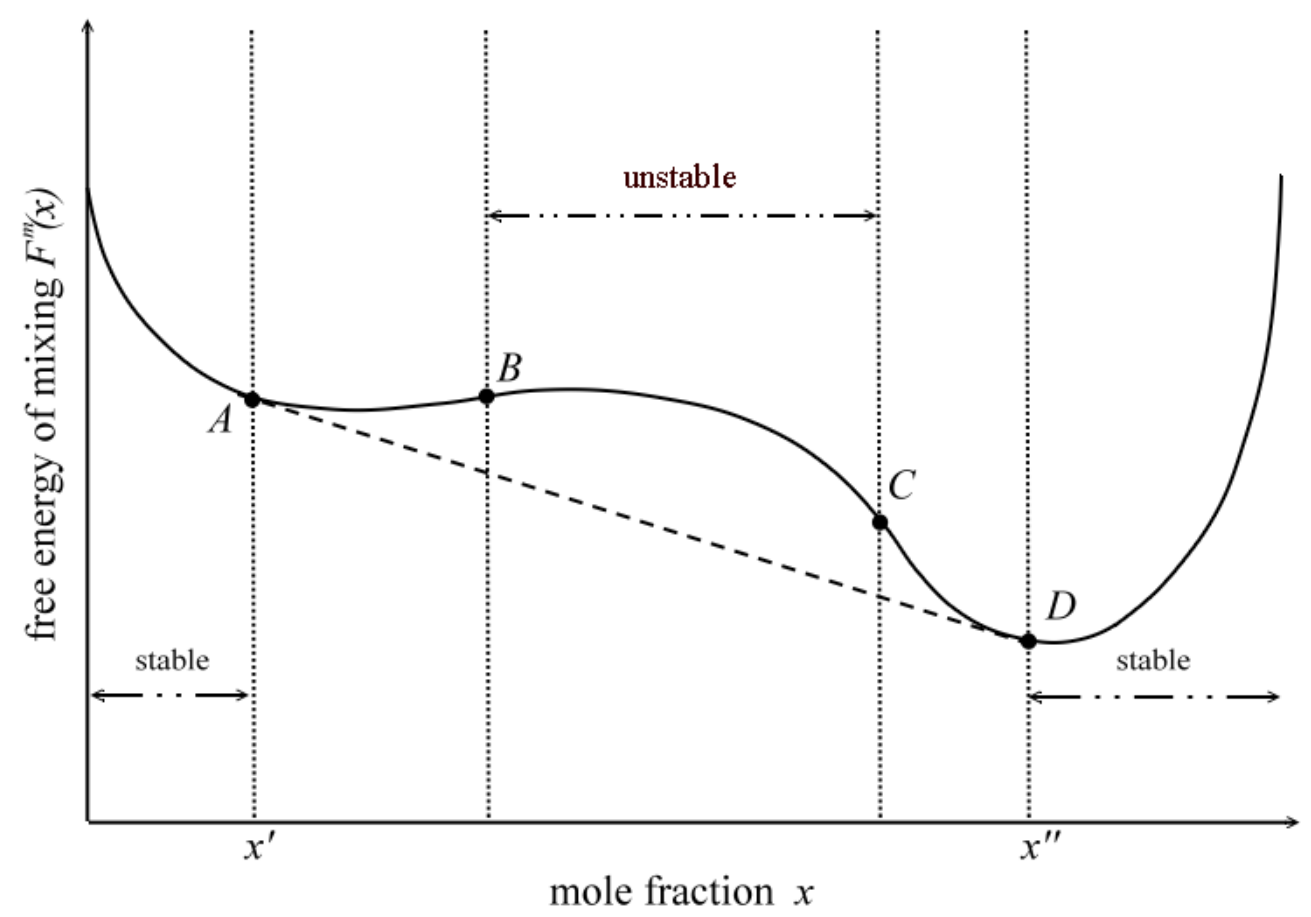

Figure 2.2: Isobar-isotherm path through the space of states of free energy of mixing $F^{m}(p, T, x)$.

\subsection{Critical fluctuations}

The correlation function of a physical parameter $X$ is represented by:

$$
g\left(\vec{r}, \vec{r}^{\prime}\right)=\left\langle x(\vec{r}), x\left(\vec{r}^{\prime}\right)\right\rangle-\langle x(\vec{r})\rangle\left\langle x\left(\vec{r}^{\prime}\right)\right\rangle
$$

here $x(\vec{r})$ denotes the density of the quantity $X$. Hence, $X$ can be expressed as:

$$
X=\int \mathrm{d}^{3} r x(\vec{r})
$$

The function $g\left(\vec{r}, \vec{r}^{\prime}\right)$ represents the degree of correlation between the values of $X$ at $\vec{r}$ and at $\vec{r}^{\prime}$. The variables in Eq.(2.4) can be replaced by the particles density $x(\vec{r}) \rightarrow n(\vec{r})$ and the number of particles $X \rightarrow N$. In other words, with increasing distance between the densities of particles $n(\vec{r})$ and the densities of $n\left(\overrightarrow{r^{\prime}}\right)$ the correlation decreases. This behavior can be written as: 


$$
\left\langle n(\vec{r}) n\left(\overrightarrow{r^{\prime}}\right)\right\rangle \stackrel{\left|\vec{r}-\overrightarrow{r^{\prime}}\right| \rightarrow \infty}{\longrightarrow}\left(\frac{N}{V}\right)^{2} .
$$

An expression, which describes this kind of behavior and its temperature dependence, has been presented by Ornstein and Zernike (1914) [29]:

$$
g\left(\vec{r}, \vec{r}^{\prime}\right)=\text { const } \cdot \frac{1}{\left|\vec{r}-\vec{r}^{\prime}\right|} \cdot \exp \left(-\frac{\left|\vec{r}-\overrightarrow{r^{\prime}}\right|}{\xi(T)}\right)
$$

Equation (2.7) represents the so-called Ornstein-Zernike-behavior. It includes the correlation length $\xi(T)$, which measures the strength of loss in correlation. This characteristic length scale is an important parameter, within the framework of critical phenomena of second order phase transitions. $\xi(T)$ divergences near the critical point that is at $T \rightarrow T_{c}$ follows $\xi(T) \rightarrow \infty$. Here $T_{c}$ denotes critical temperature. This behavior is described by the term critical fluctuations. These fluctuations tend to mask the individual characteristics of particle interactions. Moreover, in the range of critical fluctuations striking similarity of systems emerges which are otherwise quite different. This behavior is characteristic for critical phenomena.

\subsection{Critical phenomena}

The similarity of different systems mentioned in Section (2.2) can be described by universal power laws which determine the thermodynamic and transport properties close to a critical point.

\subsubsection{Critical exponents}

In order to study the critical behavior in different systems it is convenient to use the socalled reduced temperature:

$$
\varepsilon \equiv \frac{\left|T-T_{c}\right|}{T_{c}}
$$

When the temperature $T$ of a system is close to its critical temperature $T_{c}$, some relevant parameters $\mathfrak{F}$ follow a power law:

$$
\mathfrak{F}(\varepsilon)=a \varepsilon^{\varphi}\left(1+b e^{x}+\ldots\right),
$$

with $x>0$. At $\varepsilon \rightarrow 0$, that is $T \rightarrow T_{c}$, all terms except the 1 in the brackets disappear. 
Therefore, $\mathfrak{F}$ satisfies the power law:

$$
\mathfrak{F}(\varepsilon) \sim \varepsilon^{\varphi},
$$

with $\varphi$, denoting the critical exponent for the particular variable $\mathfrak{F}$.

\subsubsection{Static scaling hypothesis}

In the course of the last fifty years theoretical and experimental investigations have been done to develop and to proof the hypothesis of universality. In 1965 Widom [12] postulated the so-called scaling hypothesis, which is based on the assumption, that the singularities of different thermodynamic quantities near the critical point are represented by their generalized homogenous functions. When $F$ denotes the free energy and $\varepsilon$ the reduced temperature, it follows:

$$
F(\lambda \varepsilon)=g(\lambda) F(\varepsilon)
$$

It means, when the parameter $\lambda$ scales the reduced temperature $\varepsilon$, than $\lambda$ likewise scales the function of $\varepsilon$. Furthermore, if $\mu$ is an additional scaled variable of the function $g$, than from Eq.(2.11) follows the relation:

$$
F(\lambda(\mu \varepsilon))=g(\lambda) g(\mu) F(\varepsilon)=g(\lambda \mu) F(\varepsilon) .
$$

Comparison of the factors of the function of $F(\varepsilon)$ implies that the relation $g(\lambda) g(\mu)=$ $g(\lambda \mu)$ is only valid when $g$ follows a power law. Hence, Eq.(2.11) can be written as:

$$
F(\lambda \varepsilon)=\lambda^{a} F(\varepsilon), \text { here is } a \text { the degree of homogeneity. }
$$

From such mathematical considerations follows that differentials as well as integrals of homogenous functions are again homogenous functions. Consequently, it can be assumed that all thermodynamical quantities, which are derived from the free energy $F$, can be represented by power laws. Furthermore, the static scaling hypothesis provides also relations between the critical exponents of different parameters (see Table (2.1), for the meaning of the symbols):

$$
\begin{aligned}
& 2-\tilde{\alpha_{0}}=\beta(\delta+1) \\
& 2-\tilde{\alpha_{0}}=\tilde{v} \\
& 2-\tilde{\alpha_{0}}=\gamma+2 \beta
\end{aligned}
$$




\begin{tabular}{cll}
\hline \hline exponent & order-parameter & relation \\
\hline$\tilde{\alpha_{0}}$ & $C_{V}$ specific heat capacity & $C_{V} \sim \varepsilon^{-\tilde{\alpha}_{0}}$ \\
$\beta$ & $\rho_{2}-\rho_{1}$ density & $\rho_{2}-\rho_{1} \sim \varepsilon^{\beta}$ \\
$\gamma$ & $\kappa_{T}$ compressibility & $\kappa_{T} \sim \varepsilon^{-\gamma}$ \\
$\delta$ & $\left(p-p_{c}\right)$ pressure & $\left(p-p_{c}\right) \sim\left|\rho_{2}-\rho_{1}\right|^{\delta}$ \\
$\tilde{v}$ & $\xi$ correlation length & $\xi \sim \varepsilon^{-\tilde{v}}$ \\
$\sigma$ & $g(\vec{r}, t)$ correlation function & $g(\vec{r}, \varepsilon) \sim|\vec{r}|^{-(d-2+\sigma)}$ \\
\hline \hline
\end{tabular}

Table 2.1: Various critical relations and their order-parameters.

In 1971 the renormalization group method has been developed by Wilson [15] to calculate the critical exponents. This theory showed that the critical exponents depend only on the spacial dimensionality $d$ and the number $n$ of components of a system. Moreover, the essential message of those considerations was that phase transitions with the same dimensionality of the order parameter belong to the same universality class. Various critical exponents of systems and their order-parameter are represented in Table (2.1).

\subsubsection{Dynamic scaling hypothesis and critical slowing down}

In different investigations it has been found that, close to the critical point, various properties of relevant systems follow power laws, so that their thermodynamic properties diverge or vanish at the critical point. According to the above considerations, the static scaling hypothesis takes into account the growing of characteristic length of a system near the critical point. However, another important observation is that all transport phenomena undergo a slowing down, caused by the increase of the correlation length $\xi$. Consequently, it can be assumed that, in addition to the characteristic length, there exists a characteristic time scale. The dynamic scaling hypothesis, which describes the phenomenon of so-called critical slowing down, was first introduced by Ferrell in 1967 [32] and was subsequently generalized for magnetic systems by Halperin and Hohenberg (1969) [33], [34]. The hypothesis implies that, when the temperature $T$ of a system approaches the critical temperature $T_{c}$, the relaxation time $\tau_{\xi}$ is governed by $\xi^{\tilde{v}}$. Here $\tilde{v}$ is the exponent of the fluctuation correlation length, Table (2.2). With the life time of fluctuations, given by $1 / \tau_{\xi}=\Gamma$, and using generalized homogenous functions it is possible to to express $\Gamma$ as a generalized homogenous function of the wave vector $q$ and the reciprocal correlation length of the critical fluctuations $\xi^{-1}$ : 


\begin{tabular}{cll}
\hline \hline exponent & value & variable \\
\hline$\tilde{\alpha_{0}}$ & 0.11 & heat capacity $C_{V}$ \\
$\beta$ & 0.33 & order-parameter $\sigma$ \\
$\gamma$ & 1.24 & osmotic susceptibility $\chi_{T}$ \\
$\delta$ & 0.057 & combination of $\tilde{\alpha_{0}} / Z_{0} \times \tilde{v}$ \\
$\tilde{v}$ & 0.63 & correlation length $\xi$ \\
$Z_{\eta}$ & 0.065 & viscosity $\eta$ \\
$Z_{0}$ & 3.05 & dynamic critical exponent $\Gamma$ \\
\hline \hline
\end{tabular}

Table 2.2: Various static and dynamic exponents used in this work.

$$
\Gamma=f\left(q, \xi^{-1}\right) \text { with } f\left(\lambda q, \lambda \xi^{-1}\right)=\lambda^{z} f\left(q, \xi^{-1}\right)
$$

here is $z$ the degree of homogeneity. With the assumption $\lambda=q^{-1}$ and the implementation of the relation $\Omega(q \xi)=f\left(1,(q \xi)^{-1}\right)$ it is possible to scale the relaxation rate $\Gamma$ :

$$
\Gamma=q^{z} \Omega(q \xi)
$$

The function $\Omega(q \xi)$ is the so-called dynamic scaling function of the variables $q$ and $\xi$. This function plays an important role in the treatment of critical dynamic phenomena. Within the scope of renormalization group theory of critical phenomena it is possible to calculate specific values of the critical exponents. The results of these calculations, which have been done by Gillou [35] for static critical exponents and Burstyn and Sengers [36] for dynamic critical exponents, are shown in Table (2.2).

The development of dynamic scaling theories is a continuous process and undergoes permanent corrections and improvements ${ }^{1,2}$. In this section the essential features of dynamic scaling hypothesis have been presented which refer to binary fluids. In the case of the ternary fluids, dependent on the relevant type of phase diagram, the critical exponents have to be renormalized.

${ }^{1}$ corrections of the critical viscosity has been published in [39]. The most recent value for $Z_{\eta}$ is $0.0679 \pm$ 0.0007

${ }^{2}$ the exponent $\delta$ is often used within the framework of Bhattacharjee-Ferrell theory for the critical amplitude $S_{B F}$ of sound attenuation; see Table (2.2) 


\subsubsection{Renormalization of critical exponents}

Taking a closer look at literature of last decades which deals with the theoretical and experimental investigations of critical behavior in three-component fluids it turns out that the binary fluids conception has to be adjusted to apply to ternary fluids. Adjustment can be made by renormalization of critical exponents. Bak and Goldburg 1969 [37], [38], have performed light scattering measurements. They found larger critical exponents for the osmotic susceptibility in the critical ternary system bromobenzene-ethanol-water. Based on these results Fisher proposed a renormalization of critical exponents from calculations of the free electron Ising model [9], [10]. His idea was to keep the formalism developed for binary fluids and to consider the third component as an "impurity" of the system. The free energy of a binary liquid system is given by $F=F_{0}(T, h)$, where $h$ is the field that corresponds with the other thermodynamic parameters. In the case of ternary fluids the free energy is determined by $F\left(T, h, h_{3}\right)$, where $h_{3}$ is the field which is coupled to the "impurity" with concentration $x_{3}$. A more detailed description can be found in the papers by Müller [40], [41].

\subsubsection{Critical opalescence and equal volume criterions}

Critical opalescence is a phenomenon in liquids close to their critical point. A normally transparent liquid appears milky due to density fluctuations at all possible wavelengths. In 1908 Smoluchowski [42] was the first one who connected density fluctuations with the opalescence. In 1910 Einstein [43] showed the relationship between critical opalescence and Rayleigh scattering. Since then, critical opalescence is one of the most important indications for the existence of a critical point. However, another substantial criterion for the existence of a critical point is the so-called equal volume criterion. Only when the volumes of considered components are equal when approaching the consolute point, that point can be assumed to be a critical point. According to both criterions it is possible to determine the critical point visually.

\subsection{Phase diagrams}

The correct knowledge of the phase diagrams of the critical systems under consideration is essential for measurements at and close to the critical point.

\subsubsection{Phase diagrams for binary liquids at constant pressure}

It is common practice to present the coexistence curve of a binary mixture in a $T-x$ diagram as is shown in Fig.(2.3), where $T$ denotes the temperature and $x$ denotes the mole fraction of one constituent. Fig.(2.3) demonstrates the common types of phase diagrams. 


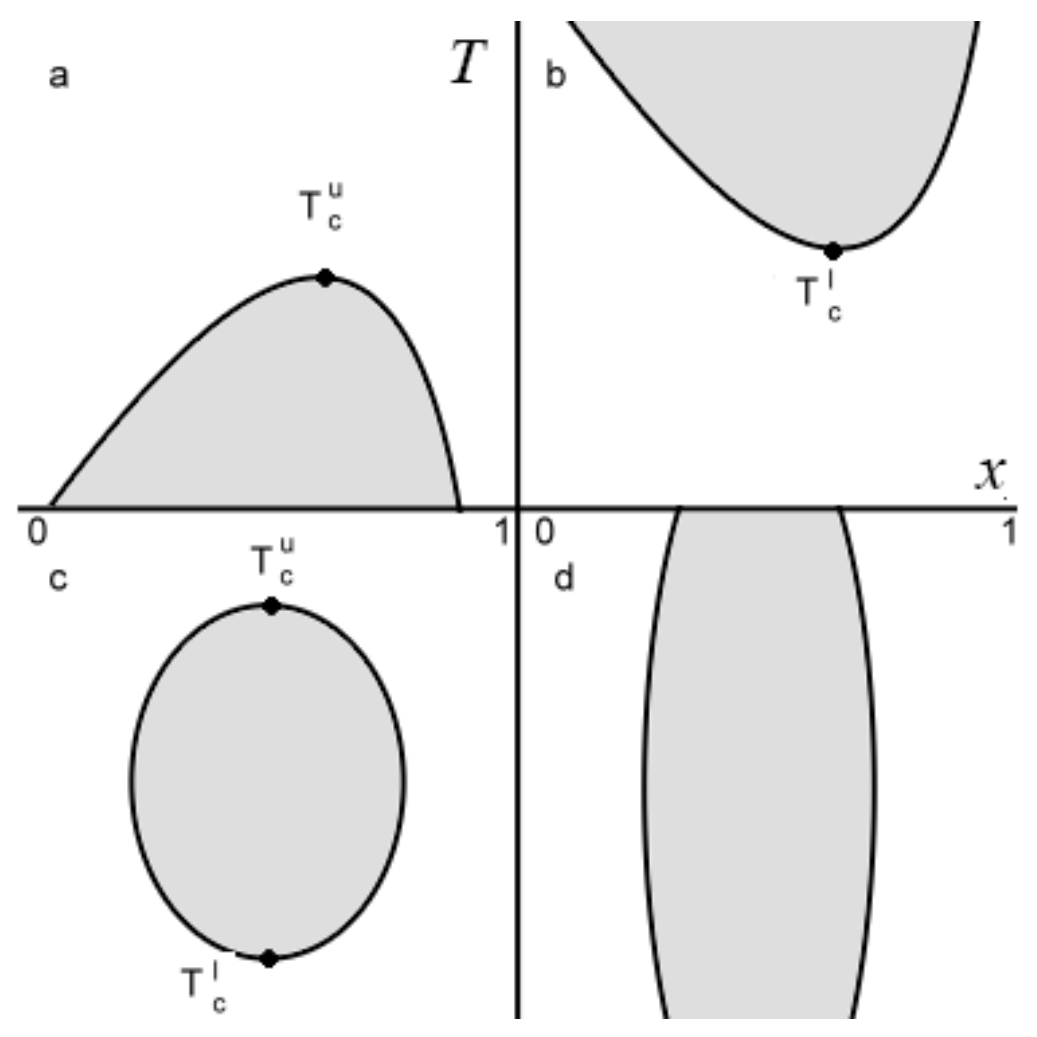

Figure 2.3: $T-x$-Types of binary phase diagram at constant pressure.

One can distinguish between open miscibility gaps (a,b,d) and closed miscibility gaps (c). In the present work only phase diagrams of type (a) and (b) play a role. Phase diagrams of type (a) with upper critical point $T_{c}^{u}$ can be found when alcohols, $n$-alkanes, as well as nitro-benzene and nitro-alkanes are involved. Phase diagrams of type (b) with lower critical point $T_{c}^{l}$ are characteristic of aqueous mixtures.

\subsubsection{Phase diagrams of ternary mixtures at constant pressure}

Ternary systems are made of three constituents. Let us denote the three constituents by $A$, $B$, and $C$. The mole fractions of the constituents are related to one another:

$$
\sum x_{i}=1 \Longrightarrow x_{A}+x_{B}+x_{C}=1
$$

where $x_{i}$ denotes the mole fraction of the constituents $A, B$ or $C$. The diagrams, as presented in Fig.(2.4) are three-dimensional but for ease of drawing and interpretation it is convenient to handle them by considering the isotherm in two dimensions. Along the 


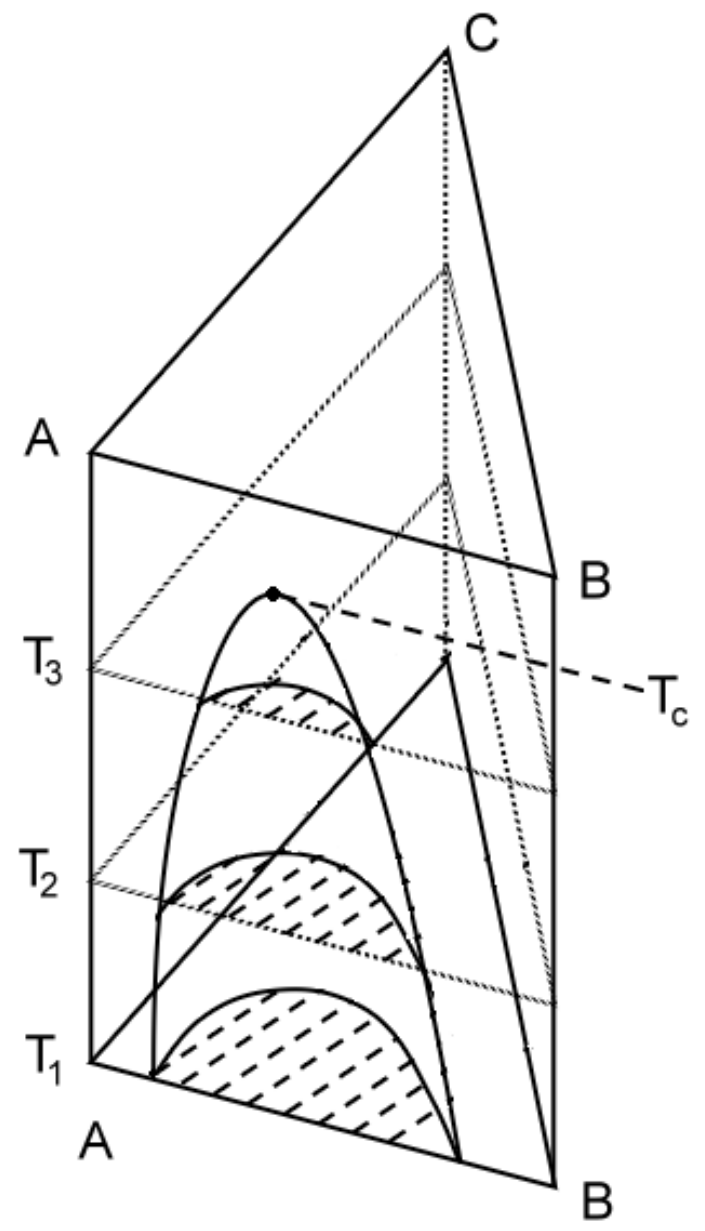

Figure 2.4: Ternary phase diagram at constant pressure.

line connecting two constituents the mole fraction of the third one must be zero. At any vortex, the mole fraction of one constituent is 1.0 while that of both others zero. An example of such isothermal diagram is shown in Fig(2.5). Obviously, the thermodynamics of ternary mixtures is more complicated as that of binary mixtures. The stability criterions discussed in Section (2.1) have to be extended to include the third component. Details of calculations and the underlying theory are given in the paper by Sadus [44]. Here only a brief outline about the conditions and criterions of the existence of a critical point in a ternary liquid system is presented. The third constituent makes it necessary to extend the before mentioned criterion of stability by an additional quantity, the diffusion coefficient $D_{0}$ : 


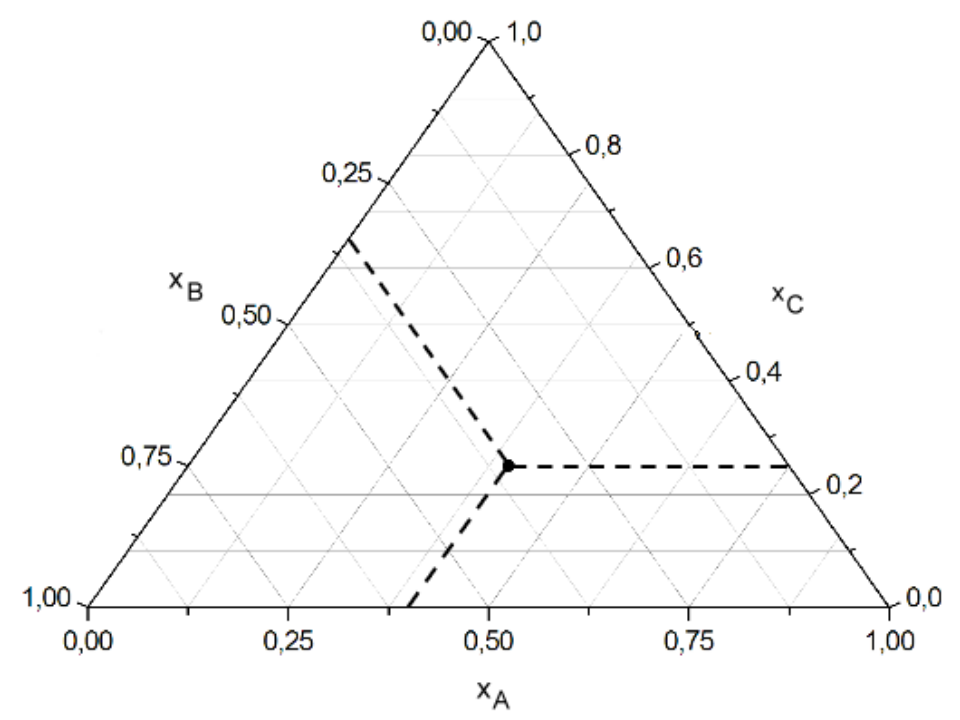

Figure 2.5: Example of an isotherm for a phase diagram of a ternary mixture: The mixing point, represented by the dot, is composed of the components $A$ with $x_{A}=0.4, B$ with $x_{B}=0.35$ and $C$ with $x_{C}=0.25 ., \sum x_{i}=1$.

$$
D_{0}=\operatorname{det} F=\left|\begin{array}{ll}
\left(\frac{\partial^{2} F}{\partial x_{A}^{2}}\right)_{x_{B}} & \left(\frac{\partial^{2} F}{\partial x_{A} \partial x_{B}}\right) \\
\left(\frac{\partial^{2} F}{\partial x_{B} \partial x_{A}}\right) & \left(\frac{\partial^{2} F}{\partial x_{B}^{2}}\right)_{x_{A}}
\end{array}\right|=\left(\frac{\partial^{2} F}{\partial x_{A}^{2}}\right)_{x_{B}}\left(\frac{\partial^{2} F}{\partial x_{B}^{2}}\right)_{x_{A}}-\left(\frac{\partial^{2} F}{\partial x_{A} \partial x_{B}}\right)^{2}=0,
$$

while $F=U-T S$ and the condition for the third constituent follows from Eq.(2.19) and is $x_{C}=1-x_{A}-x_{B}$. However, due to the reduction of the stability area and the equilibrium area to only one line, the following relation has to be fulfilled at the critical point:

$$
P_{c_{0}}=\left|\begin{array}{ll}
\left(\frac{\partial D_{0}}{\partial x_{A}}\right)_{x_{B}} & \left(\frac{\partial D_{0}}{\partial x_{B}}\right) \\
\left(\frac{\partial^{2} F}{\partial x_{B} \partial x_{A}}\right)^{2} & \left(\frac{\partial^{2} F}{\partial x_{B}^{2}}\right)_{x_{A}}
\end{array}\right|=\left(\frac{\partial D_{0}}{\partial x_{A}}\right)_{x_{B}}\left(\frac{\partial^{2} F}{\partial x_{B}^{2}}\right)_{x_{A}}-\left(\frac{\partial^{2} F}{\partial x_{A} \partial x_{B}}\right)\left(\frac{\partial D_{0}}{\partial x_{B}}\right)_{x_{A}}=0 .
$$

With the aid of Eq.(2.21) and taking the free energy $F\left(x_{A}, x_{B}, T\right)$ as well as the so-called Porter-attempt [40] into account, it is possible to assess the shape and the position of the critical line. A considerable diversity of critical equilibria can potentially be observed in a ternary mixture. Fig.(2.6) shows the existing types of phase diagrams, based on phenomenological interpretation of models of critical systems. In this thesis type $2 \mathrm{a}$ diagrams are important and are thus considered in more detail. Diagrams of type 2a result from mixing of two binary upper-critical-point mixtures with a common component. 

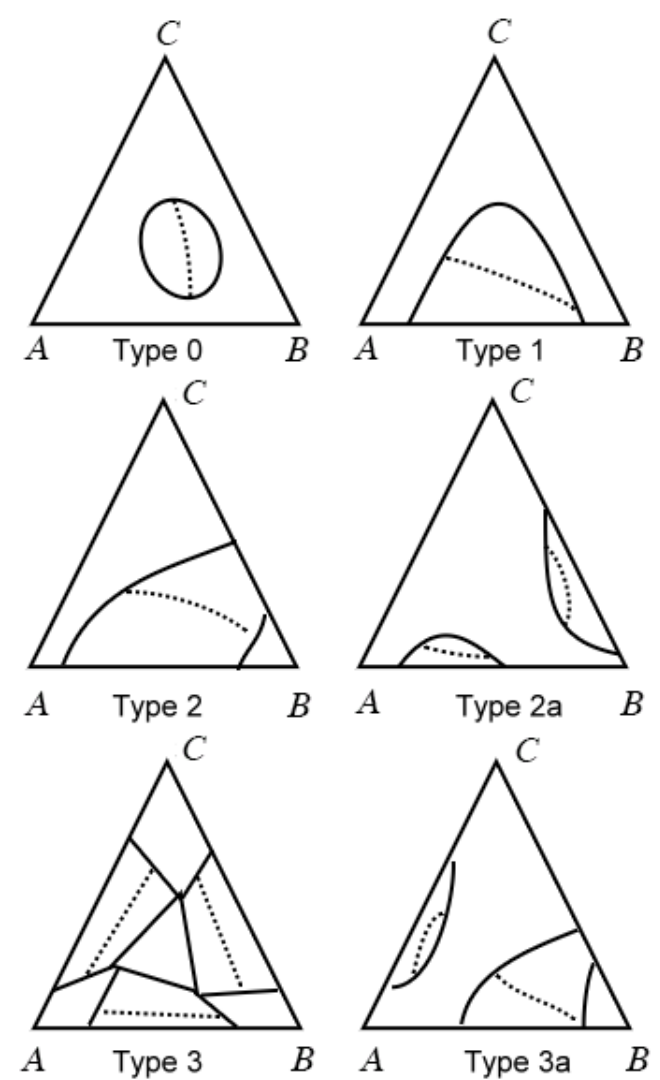

Figure 2.6: Phase diagrams for ternary liquids at constant pressure. The dashed lines show equilibrium tie lines and the full lines binodales.
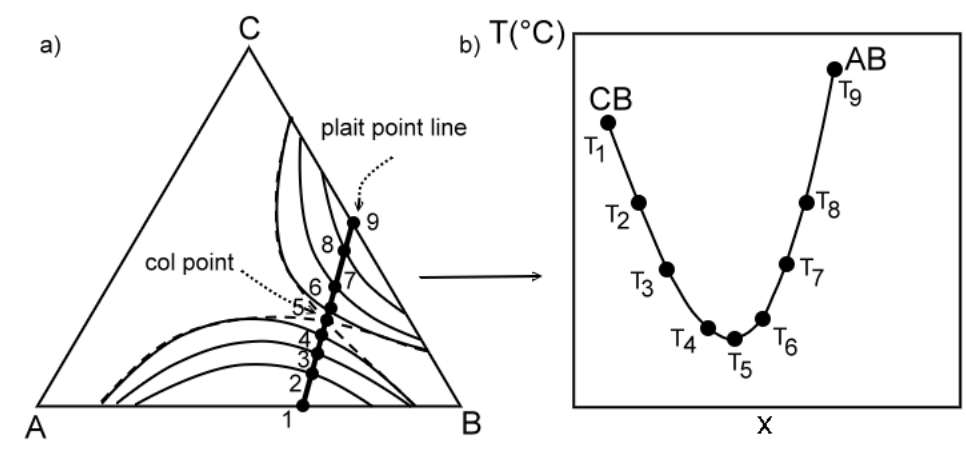

Figure 2.7: An hypothetical phase diagram of Type 2a of the ternary system $A B C: a)$ Points $(\bullet)$ refer to the line of plait points. Different isothermal binodal curves are represented at temperatures $T_{1}, \ldots, T_{2}$. b) The plait point line as a function mole fraction of $A$, from the binary system $C B$ to $A B$. 
The mixing behavior of ternary systems of type $2 \mathrm{a}$ is illustrated in Fig.(2.7). This kind of particular class of ternary liquid systems has been first found by Francis [45] in 1953. However, at constant pressure one can follow the line of so-called plait points between the demixing points of two limiting binary liquid systems. In the the case of Fig.(2.7(a)) it is the binary system $C B$ and the binary system $A B$. Each of these plait points represents the critical consolute point of a critical composition of the ternary system $A, B$ and $C$. At these points criterions for critical behavior like the equal-volume criterion or critical opalescence are fulfilled. In certain temperature ranges, the hypothetical systems under consideration, have two separate binodal lines. On lowering the temperature the two lines coincide at both plait points. Consequently, a new significant point appears, the so-called col point or saddle point. This behavior emerges when two conditions are satisfied. First, in the range of temperatures considered two components are miscible and the third one is partially miscible with both others up to the respective binary critical solution temperatures, as shown in Fig.(2.7(b)). Second, the critical consolute point must be quite close the critical point of the other binary system. In conclusion, in the triangular temperature/composition prism of the ternary system, the binodal surface is concave upwards and shows the existence of a saddle point (col point) as an extremum (in this case minimum). This happens at temperatures lower then the critical temperatures of considered binary systems, as has been indicated in Fig.(2.7(b)). Saddle points, also named col points as plait points in general fulfil critical point criteria. 



\section{Experimental Methods}

The aim of this chapter is to describe the general principles of experimental methods, which have been used in this work. The chapter is divided into four major sections. The first section (3.1) describes the general aspects of experimental set-ups, to optimize the accuracy of measurements. The second Section (3.2) deals with the dynamic light scattering (DLS) theory and experimental set-up while the third Section (3.3), focused on the broadband ultrasonic (US) theory and experimental setup. A fourth Section (3.4) presents the complementary measurement methods, like shear viscosity, density and calorimetry, which have been used to determine useful thermodynamic parameters.

\subsection{General aspects}

All technical equipment has been operated in temperature controlled $\pm 1 \mathrm{~K}$ laboratories. The specimen cells were provided with channels for circulating thermostat fluid and additionally placed in thermostatic boxes. This kind of thermostatic shielding allows to control the temperature of the measurement cells to within $0.02 \mathrm{~K}$. The temperature was measured with an error less then $0.01 \mathrm{~K}$ using Pt 100 thermometers. In order to avoid mechanical stress during the measurements, the DLS and US cells have been placed on massive granite tables. Both species of cells, the light scattering cells (sample volume $2 \mathrm{ml}$ ) as well as ultrasonic cells (samples volume between $2 \mathrm{ml}$ and $200 \mathrm{ml}$ ) have been subjected to extremely accurate cleaning procedure before use. The light scattering cells have been treated in an ultrasonic bath cleaner, filled with isopropanol, for several hours before starting a series of measurements. Finally, the cells have been dried in a vacuum oven. In the case of ultrasonic cells the cleaning and preparation procedure is somewhat different. The cells have been first flooded continuously with distilled water for several hours. Afterwards remaining water in the cells has been dissolved by methanol and the cell has been dried with the aid of nitrogen gas for about 30 minutes. The filling procedure of the ultrasonic cells has to be likewise done with care. To avoid air bubbles in the cell the substances have to be filled continuously and slowly from the bottom. The aim is to get the best contact between transducer surface and the investigated substance as well as to avoid air bubbles. Consequently, the speed of cell filling is crucial for the proper operation of the transducers. All measurements have been performed at standard pressure. 


\subsection{Dynamic light scattering}

In the last decades, light scattering techniques have been used with increasing effort for investigations of the physical properties of pure fluids and multicomponent fluids. The dynamic light scattering is a very powerful technique to determine the size of particles or to study critical fluctuations in multi-component fluids. According to the semi-classical theory, when light interacts with matter, the electric field of the light induces an oscillating electronic polarization in the molecules or atoms. With the aid of electromagnetic theory, statistical mechanics and hydrodynamics it is possible to gain information about the structural and dynamic properties of a sample. In the present work the interest is focused on the critical fluctuations.

\subsubsection{Electromagnetic scattering theory}

In the following, the underlying theory of dynamic light scattering on fluids is briefly summarized. For a more detailed description the reader is referred to specialized literature [46], [47], [48].

A typical scattering geometry for light scattering experiments is shown in Fig.(3.1). In principle, it is possible to vary the polarization of the incident light. However, with the help of scattering vector $\vec{q}$ follows from geometrical considerations, Fig.(3.1), the relationship between the wave vector $\vec{k}_{i}$ that points in the direction of the incident plane wave, and $\vec{k}_{f}$ which points in the direction of the outgoing waves ${ }^{1}$.The scattering vector is defined as $\vec{q}=\vec{k}_{i}-\vec{k}_{f}$. The amount of $\vec{q}$ is given by:

$$
q \simeq 2 k_{i} \sin (\Theta / 2)=\frac{4 \pi n_{i d}}{\lambda_{0}} \sin (\Theta / 2),
$$

with the refractive index $n_{i d}$ of the fluid, the laser wavelength $\lambda_{0}$ in vacuo, and the scattering angle $\Theta$. For a general description of interactions between a light beam and molecules, it is appropriate to study the induced dipole moment of one molecule in an electrical field. The relation between the dipole moment $\vec{p}$ of a molecule and the field $\vec{E}$ at the position $\vec{r}$ at time $t$ is given by:

$$
\vec{p}(\vec{r}, t)=\underline{\underline{\alpha}}_{L} \cdot \vec{E}_{0}(\vec{r}, t)
$$

where $\underline{\underline{\alpha}}_{L}$ denotes the polarizability tensor. In light scattering experiments the incident electromagnetic wave may be written as:

$$
\vec{E}_{0}(\vec{r}, t)=\vec{n}_{i} E_{0} \cdot e^{i\left(\vec{k}_{i} \cdot \vec{r}-\omega_{0} t\right)}
$$

\footnotetext{
${ }^{1}$ index $i$ stands for incident plane wave and index $f$ stands for the outgoing waves (towards the detector)
} 


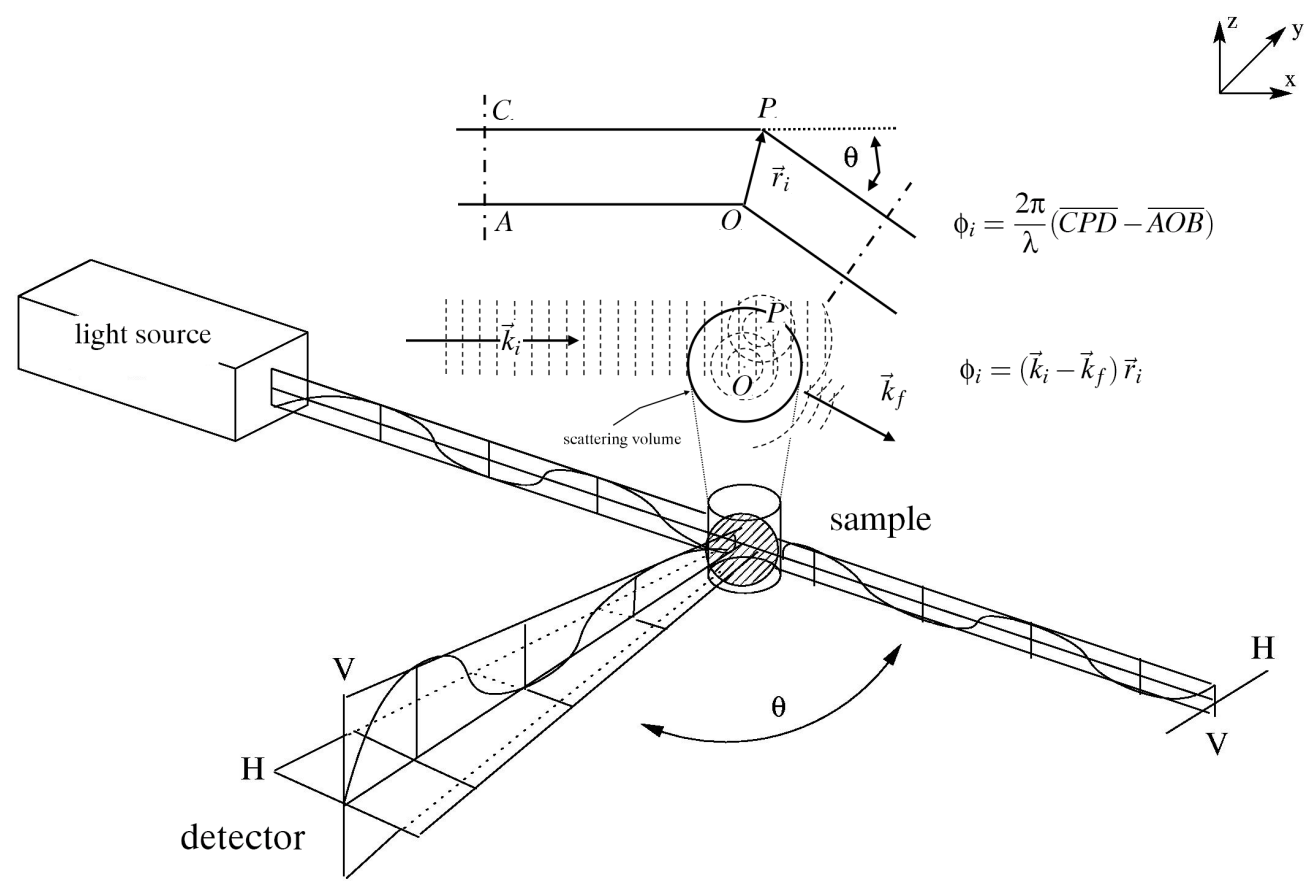

Figure 3.1: Typical scattering geometry.

with the circular frequency $\omega_{0}$ and the normal vector $\vec{n}_{i}$. The field of the electromagnetic wave induces an oscillating electronic polarization. Hence, the molecules behave as Hertzian dipoles and provide a secondary light source. From the Maxwell-equations follows the light wave propagation in a detector direction $\vec{k}_{f}$ :

$$
\vec{E}_{\text {dipole }}(\vec{R}, t)=\frac{E_{0}}{4 \pi \varepsilon_{0}} \cdot \frac{1}{|\vec{R}|} \cdot e^{i\left(\vec{k}_{f} \cdot \vec{R}-\omega_{0} t\right)}\left[\vec{k}_{f} \times\left[\alpha \cdot \hat{\vec{n}}_{i} \times \vec{k}_{f}\right]\right]
$$

with the electric field constant $\varepsilon_{0}$, and with $\vec{R}$ being the position of the detector. The Eq.(3.4) describes the electric field propagating from an elementary dipole originating from a molecule. The electrical field from all molecules in the scattering volume follows as:

$$
E(\vec{R}, t)=\sum_{j=1}^{N} E_{j}(\vec{R}, t)=C e^{\left.i k_{i} R-\omega_{i} t\right)} \cdot \int \mathrm{d} \vec{r} n\left(\vec{r}, t-\frac{|\vec{R}-\vec{r}|}{v}\right) \cdot e^{i\left(\vec{k}_{i}-k_{i} \hat{\vec{R}}\right) \vec{r}}
$$

with the constant $C=\left(\pi \alpha E_{0} / \varepsilon_{0} \lambda^{2}\right) \cdot \frac{1}{R}$ and $\hat{\vec{R}}=\frac{\vec{R}}{|\vec{R}|}$. In principle, relation (3.5) describes the scattered light completely. In practice, however, the light intensity is obtained from 
the detectors. The intensity is defined as the ensemble mean:

$$
I(\vec{R}, \omega)=\lim _{T \rightarrow \infty} \frac{1}{T}\left\langle\left|\int_{-T / 2}^{T / 2} \mathrm{~d} t E(\vec{R}, t) e^{i \omega t}\right|^{2}\right\rangle .
$$

Insertion of the Eq.(3.5) yields [49]:

$$
\begin{aligned}
I^{\prime}(\vec{q}, \omega) & =|C|^{2} \int_{-\infty}^{\infty} \mathrm{d} t \int \mathrm{d} \vec{r} e^{-i\left(\vec{q} r-\left(\omega-\omega_{0}\right) t\right)}\langle\delta n(\vec{r}, t) \delta n(\overrightarrow{0}, 0)\rangle \\
& =|C|^{2} S^{\prime}\left(\vec{q}, \omega-\omega_{0}\right) .
\end{aligned}
$$

In Equation (3.7) $\delta n$ denotes the deviation of the local particle density from the average value $(\delta n:=n(\vec{r}, t)-\langle n\rangle)$. $I^{\prime}$ and $S^{\prime}$ refer to the scattering volume where $S^{\prime}\left(\vec{q}, \omega-\omega_{0}\right)$ is the so-called dynamic structure factor. In other words, $S^{\prime}$ is the space and time Fourier transformed autocorrelation function of $\delta n$. With regard to the next section it is useful to relate Eq.(3.7) to the static scattering intensity of $I(\vec{q})$ and the static structure factor $S(\vec{q})$ :

$$
I(\vec{q})=\int_{-\infty}^{\infty} \frac{\mathrm{d} \omega}{2 \pi} I^{\prime}(\vec{q}, \omega)=|C|^{2} \int_{-\infty}^{\infty} \frac{\mathrm{d} \omega}{2 \pi} S^{\prime}\left(\vec{q}, \omega-\omega_{0}\right)=|C|^{2} S(\vec{q}) .
$$

In conclusion, an important expression for the hydrodynamic considerations results:

$$
\frac{I^{\prime}(\vec{q}, \omega)}{I(\vec{q})}=\frac{S^{\prime}\left(\vec{q}, \omega-\omega_{0}\right)}{S(\vec{q})}
$$

\subsubsection{Spectrum of scattered field - hydrodynamic considerations}

For the interpretation of the scattered spectrum of a fluid it is useful to consider the scattered field in terms of hydrodynamic approaches. In order to get access to hydrodynamics, the conservation and continuity equations have to be applied to a fluid volume element:

$$
\begin{aligned}
\frac{\partial}{\partial t} n(\vec{r}, t)+m^{-1} \nabla \vec{g}(\vec{r}, t) & =0 \\
\frac{\partial}{\partial t} \vec{g}(\vec{r}, t)+\nabla \vec{T}(\vec{r}, t) & =0 \\
\frac{\partial}{\partial t} e(\vec{r}, t)+\nabla \vec{j}(\vec{r}, t) & =0
\end{aligned}
$$


with the particle number density $n(\vec{r}, t)$, the vector of momentum density $\vec{g}(\vec{r}, t)$, the $m$ mass, as well as the energy density $e(\vec{r}, t) . \vec{T}(\vec{r}, t)$ is the stress tensor and $\overrightarrow{j_{e}}(\vec{r}, t)$ the energy flux density. In the case of fluids with negligibly small viscosity $\eta_{s}=0$, follows $T_{i j}^{0}=\delta_{i j} p(\vec{r}, t)$ and $\vec{j}_{e}^{0}(\vec{r}, t)=(e+p)(\vec{r}, t)$; where $p(\vec{r}, t)$ denotes the pressure and $\vec{v}(\vec{r}, t)$ is the average velocity of a particle. In general one gets an additive term, following from the heat conductivity $\Lambda$ of a liquid, for the energy flux $-\Lambda \nabla \vec{T}(\vec{r}, t)$. Finally, the expression of energy flux density is given by ${ }^{2}$ :

$$
\vec{j}_{e}^{0}(\vec{r}, t)=(e+p)(\vec{r}, t) \underline{-\Lambda \nabla \vec{T}(\vec{r}, t)} .
$$

However, in the general case when the viscosity of fluid is not neglected, the expression of momentum density has to be completed to account for the volume viscosity $\eta_{V}$ and the shear viscosity $\eta_{s}$. Consequently:

$$
T_{i j}(\vec{r}, t)=\delta_{i j} p(\vec{r}, t)-\eta_{s}\left\{\frac{\partial v_{i}(\vec{r}, t)}{\partial r_{j}}+\frac{\partial v_{j}(\vec{r}, t)}{\partial r_{i}}\right\}-\delta_{i j} \nabla \vec{v}(\vec{r}, t)\left(\eta_{V}-\frac{2}{3} \eta_{s}\right)
$$

follows. In conclusion, with the aid of the equations of conservation and continuity (3.11), (3.12) and (3.13), a complete mathematical mean-field description of the hydrodynamics of a liquid results. The evaluation of the relations necessitates a linearization of the coupled differential equations (3.11). Furthermore, it is usefully to express the energy density $e(\vec{r}, t)$ by a time dependent heat density:

$$
q(\vec{r}, t)=e(\vec{r}, t)-\left(\frac{\langle e\rangle-\langle p\rangle}{\langle n\rangle}\right) n(\vec{r}, t) .
$$

More details can be found in [46]. The above relations include the complete information about the scattering spectrum of a simple liquid, which can be written as:

$$
\begin{aligned}
\frac{S^{\prime}(\vec{q}, \omega)}{S(\vec{q})} & =\left(1-\frac{C_{V}}{C_{p}}\right) \cdot \frac{2 D_{T} q^{2}}{\omega^{2}+\left(D_{T} q^{2}\right)^{2}}+ \\
& +\frac{C_{V}}{C_{p}} \cdot \frac{\frac{1}{2} D_{S} q^{2}}{\left(\omega-c_{s} q\right)^{2}+\left(\frac{1}{2} D_{S} q^{2}\right)^{2}}+ \\
& +\frac{C_{V}}{C_{p}} \cdot \frac{\frac{1}{2} D_{S} q^{2}}{\left(\omega+c_{s} q\right)^{2}+\left(\frac{1}{2} D_{S} q^{2}\right)^{2}}
\end{aligned}
$$

with the heat capacities $C_{V}, C_{p}$ and thermal diffusivity coefficients $D_{T}$ and $D_{S}=D_{T}\left(\frac{C_{p}}{C_{V}-1}-\right.$

\footnotetext{
${ }^{2}$ underlined quantities denote additional terms
} 
1) $+D_{l}$, where $D_{l}$ is the sound attenuation constant, $|\vec{q}|=q$ and the sound velocity $c_{s}=\frac{1}{m}(\partial p / \partial n)_{S}$ ( $m$ denotes mass). Eq.(3.15) is a heuristic formula for the spectrum of light scattered by simple fluid and can be considered as a relation between Eq.(3.10), with the static and dynamic structure factor and the thermodynamic parameters of a fluid. The shape of the spectrum is shown in Fig.(3.16). The frequency dependence in Eq.(3.15) is that of a Lorentz function:

$$
f(\omega)=\frac{2 \Gamma_{R}}{\left(\omega-\omega^{\prime}\right)^{2}+\Gamma_{R}^{2}}
$$

Another important expression for the evaluation of DLS data is given by the Einstein-

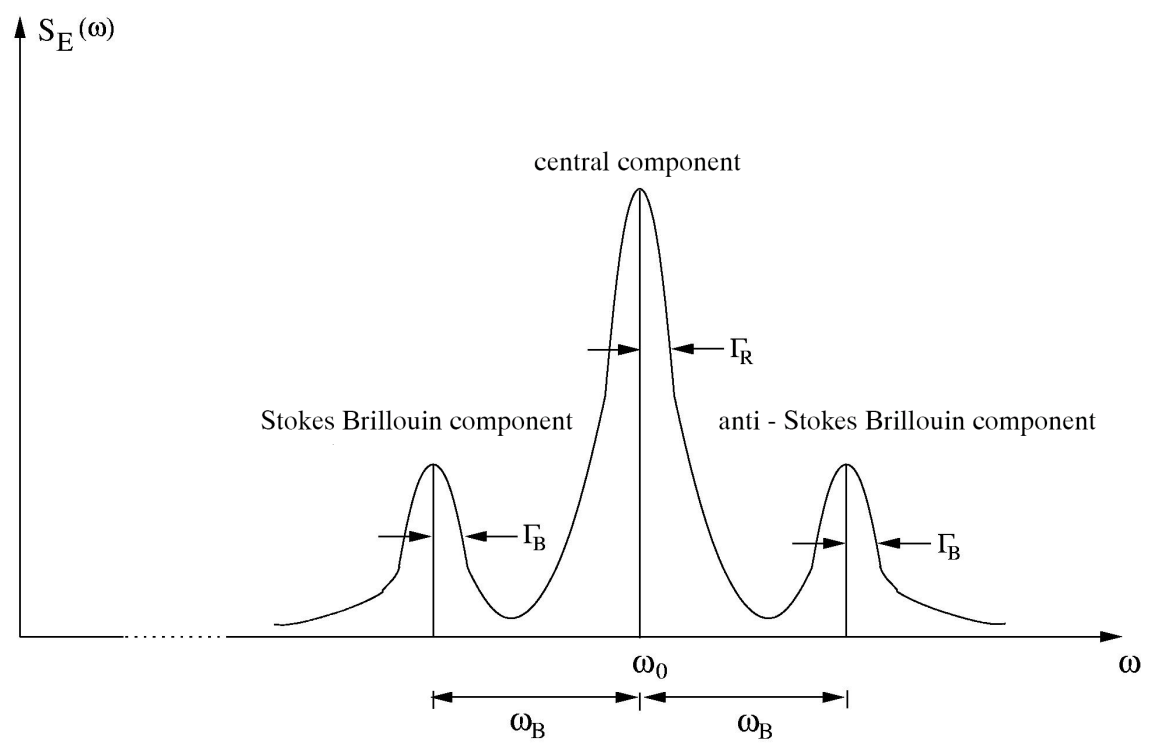

Figure 3.2: Spectrum of Scattered Light: Spectrum for the light scattered by thermal fluctuations in liquids according to Eq.(3.15).

Stokes relation [50], [51], which relates the diffusion coefficient $D$, the shear viscosity $\eta_{s}$ and to the radius $r$ of a particle:

$$
D=\frac{k_{B} T}{6 \pi \eta_{s} r}
$$

Eq.(3.17) holds for simple fluids. In the case of multi-component critical systems the diffusion has to be considered within the framework of the mode-coupling theory. Modecoupling theory yields a similar expression for the critical part of mutual diffusion coefficient: 


$$
D \simeq \frac{k_{B} T}{6 \pi \eta_{s} \xi}
$$

with the correlation length $\xi$. More detailed descriptions of the mutual diffusion coefficient and its corrections within the scope of dynamic scaling and crossover theory will be presented in Chapter (4).

\subsubsection{Data evaluation of dynamic light scattering}

The central quantity of the evaluation of correlation spectroscopy is the decay time also named the correlation-time $\tau_{c}$. Unfortunately, it is not possible to detect this frequency with classical optical methods. As a consequence, it is substantial to use optical mixing techniques, like homogenous mixing, heterogenous mixing (homodyne and heterodyne techniques) and the self-beating method. The last one, has been used in present thesis and will be described below.

\subsubsection{Self-beating spectroscopy}

The principle of self-beating spectroscopy is based on mixing of scattered light with the original light on analyzing the resulting signal with low-frequency intermediate signal. This is realized in three steps.

- Consider a signal of field strength $\vec{E}(\vec{R}, \omega)$ with spectrum $A_{i}$, that has to be shifted into the lower frequencies. This happens with the aid of a local-oscillator signal. After this mixing procedure, it is possible to describe the scattered light with the help of convolution integral of the incoming spectrum $\left(A_{p}\right.$, spectrum of the photon flux):

$$
A_{p}(\omega) \propto \int_{-\infty}^{\infty} A_{i}\left(\omega^{\prime}\right) A_{i}\left(\omega-\omega^{\prime}\right) d \omega^{\prime}
$$

On the one side, the photon flux $i(t)$ is proportional to the light intensity, $|I(\omega)|=$ $|\vec{E}(\omega)|^{2}$. On the other side, according to Eq.(3.16), the considered Rayleigh line is of the shape of a Lorentz curve, with the the half-bandwidth $\Gamma_{R}$ and the center frequency $\omega_{0}$ :

$$
A_{i} \propto \frac{\Gamma_{R}}{\left(\omega-\omega_{0}\right)^{2}+\left(\Gamma_{R}\right)^{2}} .
$$

Hence, resulting from the convolution integral Eq.(3.19) the spectrum $A_{p}$ of the 
photon flux $i(t)$, can be expressed by:

$$
A_{p} \propto \frac{2 \Gamma_{R}}{\omega^{2}+\left(2 \Gamma_{R}\right)^{2}} .
$$

For simple fluids the half-bandwidth $\Gamma_{R}$ (see Fig.(3.16)) of the Lorenz-curve can be represented by:

$$
\Gamma_{R}=\frac{\Lambda}{\rho C_{p}} \cdot q=D_{T} \cdot q
$$

here denotes $D_{T}$ the thermal diffusivity, $\Lambda$ the thermal conductivity, $\rho$ the density, $C_{p}$, the heat capacity at constant pressure $p$ as well as $q$, the amount of the scattering vector. The next step is to find a tool to measure the spectrum. This can be realized with the aid of Correlation Spectroscopy.

- Correlation Spectroscopy: The Wiener-Khinchin theorem $\left|A_{f}(\omega)\right|^{2}=\mathcal{F}^{-1} \Phi[f(t)]$ relates the signal $f(t)$ with the amount of the spectrum $A_{f}(\omega)$. Here is $\mathcal{F}^{-1}$ the inverse Fourier transform and $\Phi[f(t)]$ is the autocorrelation function of $f(t)$. As mentioned before, the signal $i(t)$ of the photon flux is proportional to the light intensity $I(t)$. Practically, in an experiment the autocorrelation function $\Phi[i]$, which is proportional to the autocorrelation function of $\Phi[I]$, is determined. Consequently, the following expression results:

$$
\Phi[i] \propto \Phi[I] \propto \frac{\int_{-\infty}^{\infty} I(t) I\left(t+\tau_{c}\right) d t}{\int_{-\infty}^{\infty} I(t)^{2} d t} \equiv g^{(2)}\left(\tau_{c}\right) .
$$

In an experiment the interest is not focused on the intensity spectrum of the but in the spectrum of electric filed $E(t)$. Due to the well known relation $I(t)=|E(t)|^{2}$, the expression (3.23) for the electric field is given by:

$$
g^{(1)} \equiv \Phi(\vec{E})=\frac{\int_{-\infty}^{\infty} \vec{E}^{*}\left(\tau_{c}-t\right) \vec{E}(t) d t}{\int_{-\infty}^{\infty}|\vec{E}(t)|^{2} d t} .
$$

Finally, the autocorrelation function $g^{(2)}$ of the intensity $I(t)$ and the autocorrelation function $g^{(1)}$ of the field $E(t)$ are related by the so-called Siegert relation:

$$
g^{(2)}\left(\tau_{c}\right)=1+\left|g^{(1)}\left(\tau_{c}\right)\right|^{2} .
$$

In the DLS experiment one has to do with the Lorentz profile, as is according to Eq.(3.21). The autocorrelation function of this profile is given by:

$$
\Phi[i] \propto e^{-2 \Gamma_{R}|t|}=e^{-|t| / \tau_{c}} .
$$


Equation (3.26) allows to determine the half-with $\Gamma_{R}$ of Rayleigh-line from the correlation-time $1 / 2 \Gamma_{R}$ of the photon autocorrelation function.

- Photon statistics: All considerations have been done with the assumption that $i(t) \propto$ $I(t)$. In principle, this can be done for sufficiently high scattering count rates. For experiments with lower rates it is substantial to use adequate statistics. This is described in detail in [46].

\subsubsection{Technical equipment}

A typical dynamic light scattering set-up is presented in Fig.(3.3). A frequency-doubled Nd:YAG laser (1) is used as light source. The laser light passes several diaphragms (2), a polarizer (4) and a collimator lens (5) with focal length $f=100 \mathrm{~mm}$ and is then fed to the sample cell (6). The scattered light passes a microscope objective (7), a polarization analyzer (9) and the slit (10) with $(d=200 \mu \mathrm{m})$. Finally, the signal is detected by a photomultiplier (Hamamatsu Electronic, Model R647P) (12) that transforms a variation of intensity into a variation of voltage. The spectrometer is provided with a goniometer system which allow superior of scattering angle $\Theta$. The received signal is analyzed with help of a correlation card ALV-5000/E with logarithmic timescale and with 288 channels. In principle it is possible to analyze the autocorrelation function in terms of a superposition of up to four exponentials and thus four correlation times $\tau_{c}$. Hence the Rayleigh line may be considered a sum of up to four Lorentz functions. Combining the correlation time $\tau_{c}$ with the Eqs.(3.1), (3.22) and (3.17) or (3.18), it is possible to determine the radius $r$ of a particle and thus the correlation length $\xi$. 


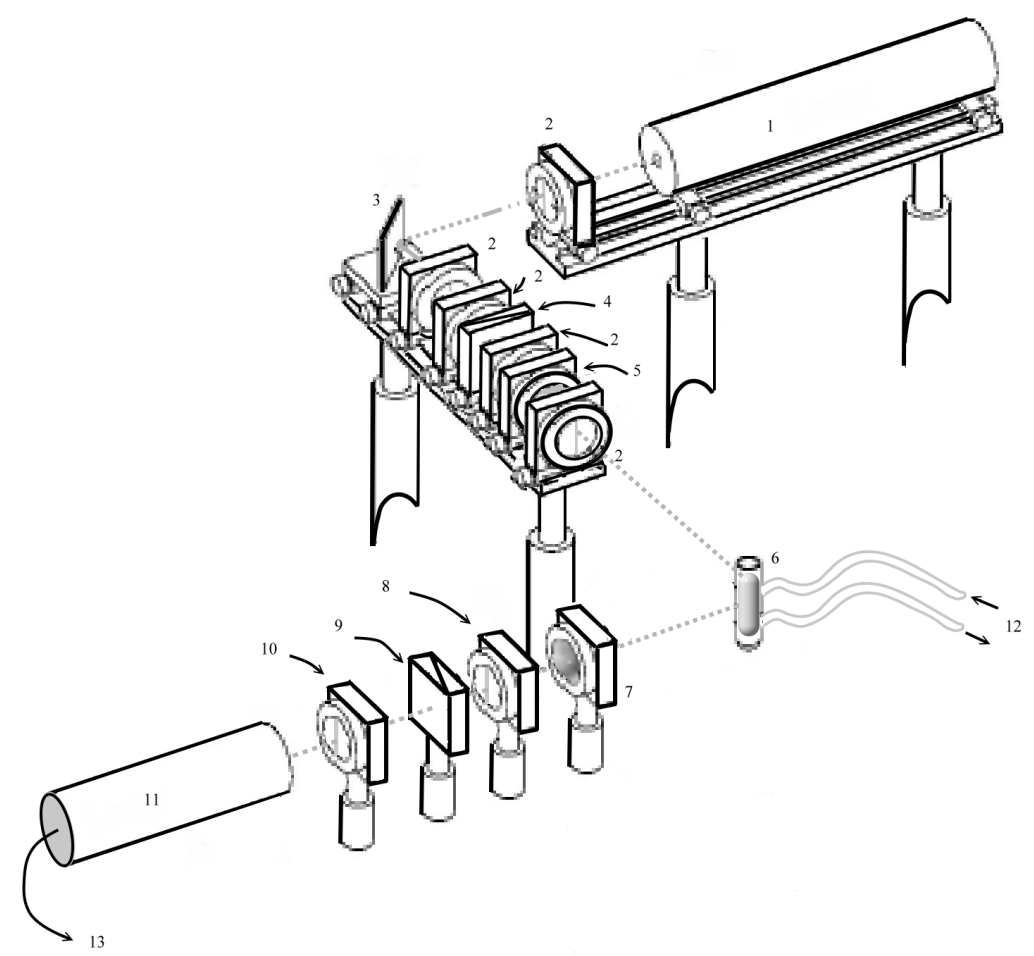

Figure 3.3: Construction of the DLS set-up: (1) frequency-doubled Nd:YAG laser ; (2) diaphragms; (3) mirror; (4) polarizer; (5) collimator lens; (6) sample cell; (7) microscope objective; (8) diaphragms; (9) analyzer; (10) slit; (11) photomultiplier (Hamamatsu Electronic, Model R647P) ; (12) thermostat channels ; (13) electronic equipment (correlation card ALV5000/E and personal computer).

\subsection{Ultrasonic techniques}

This section describes some basic principles of ultrasonic spectroscopy. Ultrasonic spectroscopy is used to study fast elementary molecular processes in liquids. It is possible to study phenomena like stoichiometrically well defined chemical equilibria, including protolysis and hydrolysis reactions, conformational changes, association mechanisms and critical fluctuations. Oscillating compressions and decompressions in an ultrasonic wave cause oscillations of molecular arrangements in the liquid. An advantage of this technique is that the amplitudes of deformations in the ultrasonic waves are extremely small. It is a non-destructive technique. The available frequency range from $80 \mathrm{kHz}$ to $5 \mathrm{GHz}$ necessitates the use of different techniques: resonator methods and variable path length methods. However, this need provides another advantage of this methods. Because of different instrumental set-ups, systematic errors are unlikely to remain unnoticed. 


\subsubsection{Classical absorption and background contribution}

Sound fields constitute temporal and spatial oscillations of the local pressure, which propagate through the liquid medium adiabatically, with its amplitude decreasing exponentially along the direction of propagation $z$ by:

$$
p(z, f, t)=p_{0} \cdot e^{-\alpha z} \cdot e^{-i \cdot 2 \pi(f t-z / \lambda)},
$$

where $\alpha$ is the attenuation coefficient, $c_{s}$ is the sound velocity in the liquid for compressional waves of frequency $f, c_{s}=\lambda \cdot f, p$ is the sinusoidally oscillating sound pressure, $p_{0}=p(z=0)$ and $i^{2}=-1$. The attenuation coefficient $\alpha$ is usually considered in ultrasonic spectroscopy. For our understanding of acoustical spectra, it is necessary to identify the physical mechanisms leading to sound attenuation in liquid systems. Oscillations of the liquid are coupled to the shear viscosity and other transport properties, which thus play an important role in compressional wave interactions. If the particle velocity is smaller than the sound velocity, the field in a viscous liquid is determined by the Navier-Stokes equations resulting in an acoustic absorption coefficient. An expression, which describes viscosity losses, is [52]:

$$
\alpha_{v i s}(f)=\frac{2 \pi^{2}}{3 c_{s}^{3} \rho}\left(4 \eta_{s}+3 \eta_{V}\right) \cdot f^{2}
$$

with:

$$
\begin{array}{rll}
\eta_{s} & : & \text { shear viscosity } \\
\eta_{V} & : \text { volume viscosity } \\
f & : \text { frequency with } f=\omega / 2 \pi \\
\rho & : \text { density } \\
c_{s} & : \text { sound velocity }
\end{array}
$$

The relation (3.28) is strongly dependent upon the properties of the medium and on the frequency. Here the properties of a medium are mainly defined by the shear and the volume viscosity. Shear viscosity has the origin in Stokes friction. Additional losses result from the thermal conductivity, which has been fist shown in 1868 by Kirchhoff [53]:

$$
\alpha_{\text {thermal }}(f)=\frac{2 \pi^{2}}{c_{s}^{3} \rho}\left(\frac{C_{p}}{C_{V}}-1\right) \frac{X}{C_{p}} f^{2}
$$

with: 
$C_{p}:$ specific heat capacity, $p=$ const.

$C_{V}:$ specific heat capacity, $V=$ const .

$X$ : coefficient of heat conductivity.

From history, it is a common practice to call the sum $\alpha_{v i s c}+\alpha_{\text {thermal }}$ as the "classic" part of the acoustic attenuation coefficient $\alpha_{\text {class }}$. In aqueous solutions the $\alpha_{\text {thermal }}$ contribution part to $\alpha_{\text {class }}$ is usually small $\left(\alpha_{\text {thermal }} \ll \alpha_{v i s}\right)$ and can be neglected. In principle the the shear viscosity $\eta_{s}$ and the volume viscosity $\eta_{V}$ in Eq.(3.28) are frequency dependent quantities. Therefore:

$$
\alpha_{v i s}(f)=\frac{2 \pi^{2}}{3 c_{s}^{3} \rho}\left(4 \eta_{s}(f)+3 \eta_{V}(f)\right) \cdot f^{2}
$$

However, it is convenient for the discussion of measured acoustical spectra to assume the frequency-independent asymptotic "background contribution" $B^{\prime}$, characterizing the total absorption at frequencies far above the experimental range. This includes the relaxation processes, occurring at frequencies well above the measuring range. Subtracting the asymptotic value from the measured absorption gives the so-called excess contribution $\left(\alpha / f^{2}\right)_{\text {exc }}$ and the excess absorption per wavelength $(\alpha \lambda)_{\text {exc }}$ :

$$
(\alpha \lambda)_{e x c}=(\alpha \lambda)-\underbrace{B}_{B^{\prime} c_{s}} f
$$

\subsubsection{Noncritical ultrasonic excess absorption}

Fast elementary molecular reactions, that are usually exhibited by ultrasonic excess absorption spectra, with the inverse relaxation time in the frequency range of measurement, are conformational changes, protolysis and hydrolysis, as well as dimerization and complexation mechanisms. Chemical equilibria are associated with Debye-type relaxation terms, exhibiting discrete relaxation times $\tau$. According to [55], the excess absorption for one relaxation process can thus be described by:

$$
R_{D}(f)=(\alpha \lambda)_{e x c}^{D}=A \cdot \frac{\omega \tau}{1+(\omega \tau)^{2}}
$$

with $\omega=2 \pi f$, and $A$ being the relaxation amplitude. Let $X$ and $Y$ represent different conformers of the same species in a dynamical equilibrium. The unimolecular reaction 
scheme is then simply given by:

$$
X \underset{k_{r}}{\stackrel{k_{f}}{\rightleftarrows}} Y .
$$

Here $k_{f}$ denotes the forward rate constant and $k_{r}$ represents the reverse rate constant. Both constant are related to the equilibrium constant $K=k_{f} / k_{r}$ and to the relaxation time of Eq.(3.32) by:

$$
\tau^{-1}=k_{f}+k_{r}
$$

In order to discuss some general characteristics of ultrasonic relaxation, an energy scheme is sketched in Fig.(3.4), where a (hypothetical) potential is given as a function of the molecular volume of the species undergoing a boat/chair conformation equilibrium like that of cyclohexane.

In Fig.(3.4) it has been assumed that the species $X$ and the species $Y$ differ from one another by the molar reaction volume

$$
\Delta V=V_{Y}-V_{X}
$$

and by the reaction enthalpy $\Delta H$. In principle, the idea of getting information about $\alpha_{\text {exc }}$ is simple. In a sound field, the energy profile oscillates around the equilibrium curve, as indicated by the dashed and dotted curves in Fig.(3.4). The autocorrelation function for thermal fluctuations of the population numbers $N_{Y(t)}$ and $N_{X(t)}$ of species $Y$ and $X$, re-

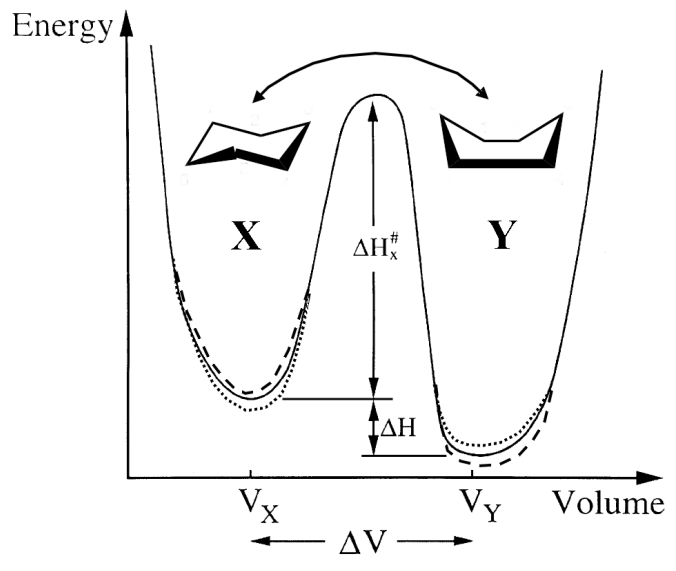

Figure 3.4: Qualitative energy profile of a chemical relaxation process it the case of cyclohexane (based on a Figure in [54]) spectively, in a given volume element and thus the autocorrelation function of the thermal fluctuations in the density $\rho$ of the sample is characterized by an exponential decay:

$$
\Phi_{\rho}(t)=\langle\rho(t) \cdot \rho(0)\rangle=\Phi_{\rho} e^{(-t / \tau)}
$$

with the autocorrelation time $\tau$. The liquid system tends to follow the oscillations in the potential curve. The transition from one conformation to another is controlled by the activation enthalpy barrier $\Delta H>R T$, establishing a finite probability for that conformational change. In accordance with the Le Chatelier principle it follows the reaction, in Eq.(3.33). 
A time lag between pressure and density in the sound field leads to a dissipation of acoustic energy with an attenuation coefficient $\alpha$ in Eq.(3.27). At $f \ll(2 \pi \tau)^{-1}$ the system has sufficient time to reach the equilibrium without significant decay. At high frequencies $f \gg(2 \pi \tau)^{-1}$, the system can not follow the rapid pressure variations. In the frequency range $f \simeq(2 \pi \tau)^{-1}$, sound energy dissipation per cycle has a maximum, leading to the characteristic profile of Debye term. The unique Debye term fits spectra pretty good in case of well defined molecular processes. Sometimes it is not possible to describe an excess absorbtion spectrum by one Debye term of the form Eq.(3.32). Especially, when different molecular processes exist in the frequency range of measurement it is mostly necessary and possible to apply a sum of Debye terms:

$$
R_{D_{i}}(f)=(\alpha \lambda)_{\operatorname{exc}}^{D_{i}}=\sum_{i=1}^{N} A_{i} \cdot \frac{\omega \tau_{i}}{1+\left(\omega \tau_{i}\right)^{2}} .
$$

Unfortunately, sometimes molecular processes lead to very complicated spectra. Con-

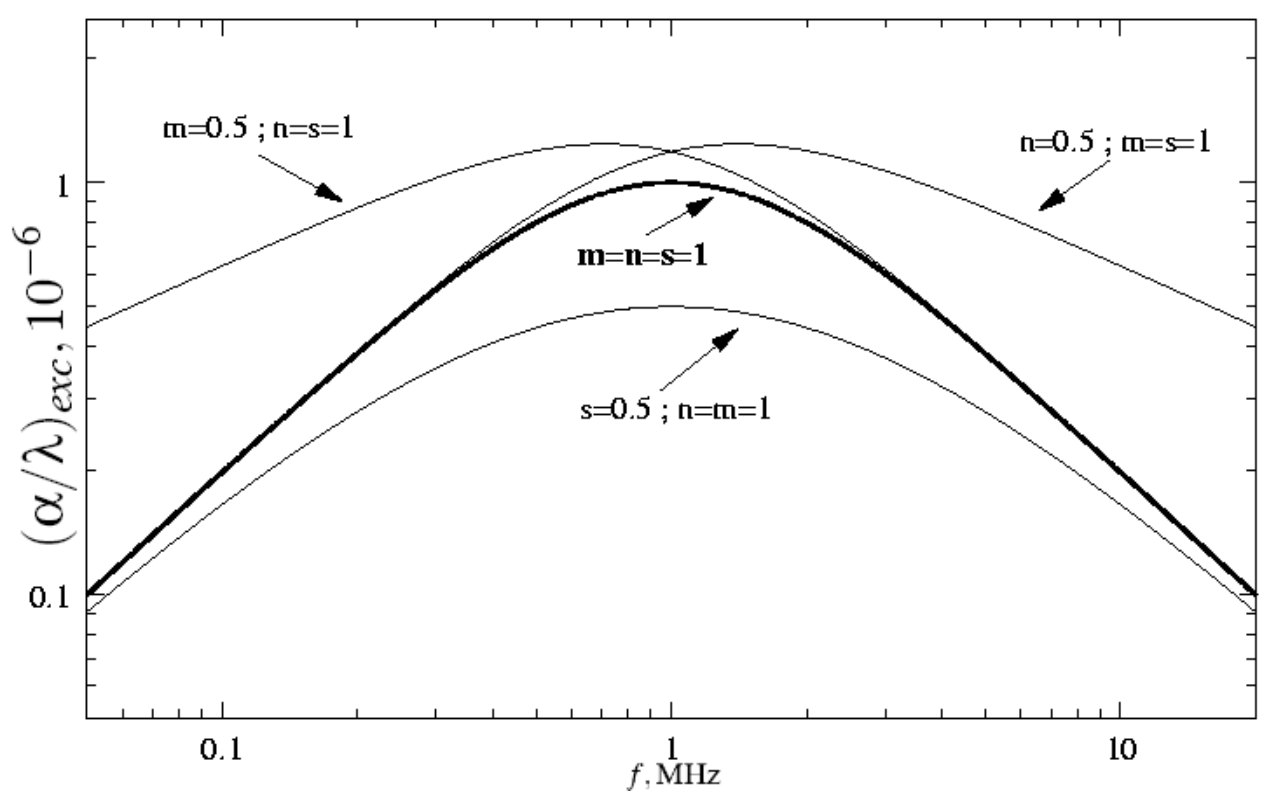

Figure 3.5: An example of the Hill function with different values of $m, n$ and $s$, according to Eq.(3.38).

sequently, they can only be regarded as a sum of Debye-terms with a particular distribution of amplitudes. Menzel at al. [58] found the relaxation spectral function, originally introduced by Hill [56], [57] discussing non-exponential decay in the polarization of dielectrics, to be favorably utilized in physical acoustics. The Hill function is given by (see 
Fig.(3.5)):

$$
R_{H}(f)=(\alpha \lambda)_{e x c}^{H}=A \frac{(\omega \tau)^{m}}{\left(1+(\omega \tau)^{2 s}\right)^{\frac{m+n}{2 s}}} \quad, \quad \text { with } m, n, s \in[0,1]
$$

where $A$ denotes an amplitude. Eq.(3.38) reflects an underlying continuous relaxation time distribution with a characteristic relaxation time $\tau$ and with parameter $m, n$ and $s$ , $(0 \leq m, n, s \leq 1)$, that determine the width and the shape of distribution function. If $m=n=s=1$ then the Hill spectral function corresponds with a Debye term, Eq.(3.32), bold plot in Fig.(3.5). With a reduced number of adjustable parameters the restricted Hill function:

$$
R_{H}^{\#}(f)=(\alpha \lambda)_{e x c}^{H^{\#}}=A \frac{(\omega \tau)^{m}}{\left(1+(\omega \tau)^{2 s}\right)^{\frac{3}{4 s}}}
$$

is then appropriate. A characteristic example for a restricted Hill function will be shown later in Chapter (5) when the critical system isobutoxyethanol-water [77] is discussed.

\subsubsection{Critical systems and total attenuation spectrum}

In the case of critical liquid systems, the ultrasonic spectra get an additional contribution, resulting from the critical fluctuations. This critical attenuation term has been treated within the framework of the Bhattacharjee-Ferrell theory. Assuming additivity of the critical contributions and the other ones, the total ultrasonic absorption spectrum, can be written as:

$$
(\alpha \lambda)=\underbrace{(\alpha \lambda)_{c}+(\alpha \lambda)_{e x c}}_{(\alpha \lambda)_{e x c}^{*}}+(\alpha \lambda)_{b g}
$$

However, because of its essential role in these investigations, the first contribution to Eq.(3.40) will be treated separately in the Chapter (4).

\subsubsection{Ultrasonic instruments}

Next sections deal with the realization of different ultrasonic methods. The ultrasonic cell consists of two piezoelectric transducers arranged parallel to each other. One of these disc-shaped transducers acts as the transmitter, the other one as receiver. The surfaces of the used transducers are coaxially plated with electrodes (chrom/gold). The sound wave is induced by the inverse piezoelectric effect. In principle two kinds of ultrasonics 


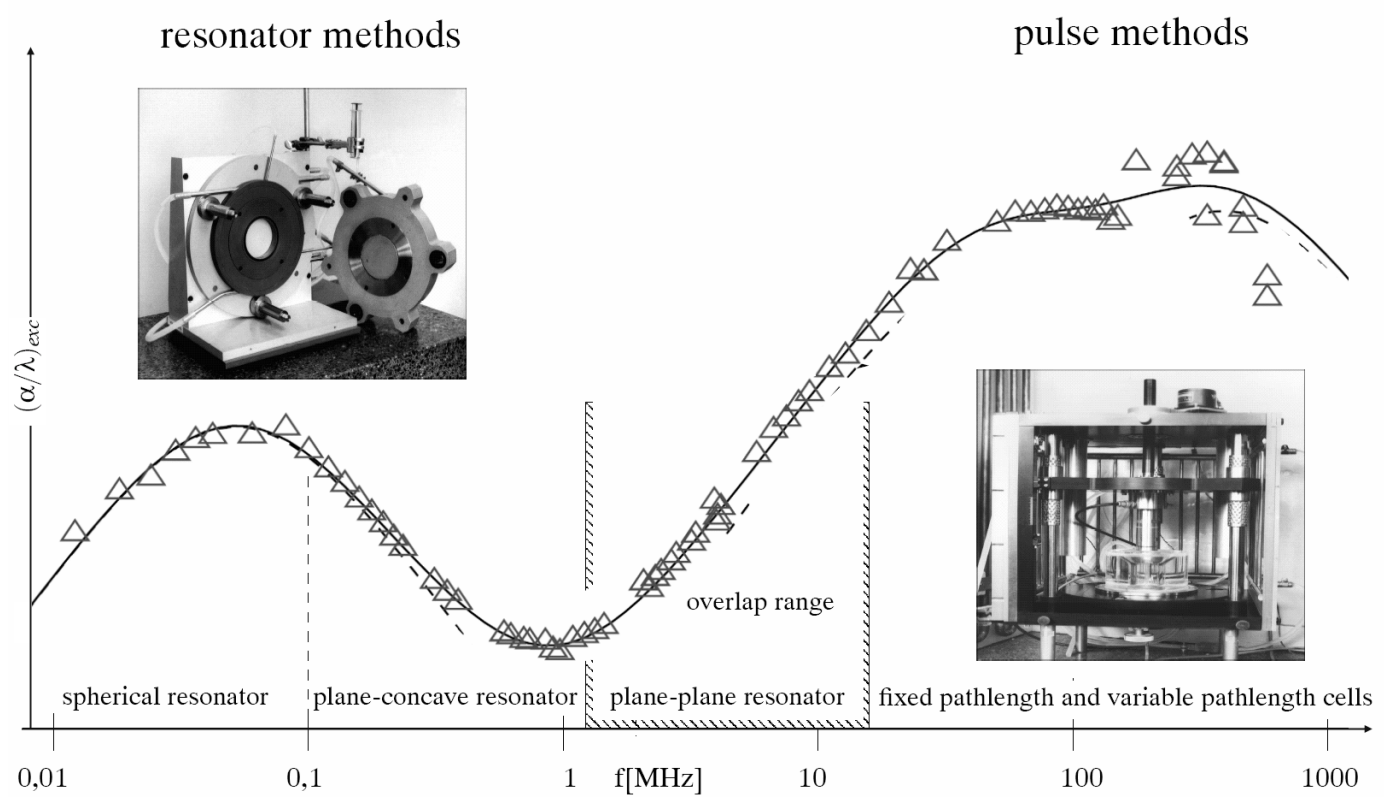

Figure 3.6: Review of broadband ultrasonic methods.

techniques were used in present work; the resonator method Section (3.3.3) and the pulse transmission method Section (3.3.4). The principle of the resonator method is based on folding the acoustic path via multiple reflections in order to obtain a resolvable amplitude decay at lower frequencies. With the help of pulse methods it is possible to make measurements of the exponential amplitude decay versus distance of propagating waves, which propagate through the liquid. Standing and the traveling wave methods overlap in a wide range and can be used to check the consistency of measured ultrasonic spectra.

\subsubsection{Resonator cells $80 \mathrm{kHz}-20 \mathrm{MHz}$}

\section{The ideal resonator}

In order to understand the functioning of a real resonator, it is appropriate to take a look at the ideal resonator. In this work cylindrically shaped ultrasonic resonator cavities are operated in compressional modes. For the consideration of an ideal resonator some assumption have to be made:

First, the ideal resonator consists of two piezoelectric planar transducers, separated by the distance $l$ and with radius $R$. The wavelength of the sound waves is $\lambda \ll R$. As a consequence, plane waves are considered. Second, energy dissipation occurs only in the fluid. Because of these assumptions it is possible to express the sound pressure at the 


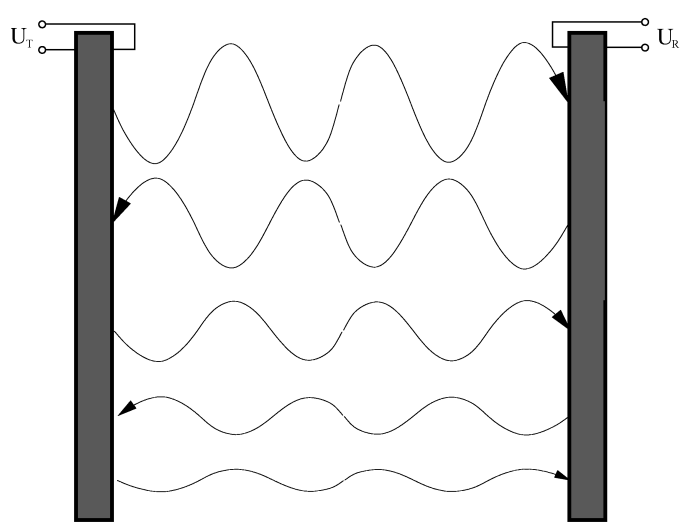

Figure 3.7: Basic principle of an ultrasonic cell.

receiver ${ }^{3}$ as a mathematical series:

$$
p_{R}=p_{0}\left(1+r_{R}\right) e^{-\gamma l} \cdot \sum_{n=0}^{\infty}\left(r_{R} r_{T} e^{-2 \gamma l}\right)^{n} .
$$

This series convergences for $r_{R} \cdot r_{T}<1$. With $p_{T}(0, t)=p_{0} \cdot e^{i \omega t}$ this yields:

$$
p_{R}=p_{0}\left(\frac{\left(1+r_{R}\right)}{e^{\gamma \cdot l}-r_{R} r_{T} e^{-\gamma \cdot l}}\right) e^{i \omega t} .
$$

Finally, the transfer function $T(f)$ results as proportionality:

$$
T(f)=\frac{U_{R}}{U_{T}} \sim \frac{p_{R}}{p_{T}}=\frac{1+r_{R}}{e^{\gamma \cdot l}-r_{R} r_{T} e^{-\gamma \cdot l}} .
$$

Its absolute value is:

$$
|T(f)| \sim \frac{\left(1+r_{R}\right)}{\sqrt{\left(e^{\alpha l}-r_{R} r_{T} e^{-\alpha l}\right)^{2}+4 r_{R} r_{T} \sin ^{2}(k l)}},
$$

with:

\footnotetext{
${ }^{3}$ index $R$ denotes the receiver and index $T$ the transducer
} 


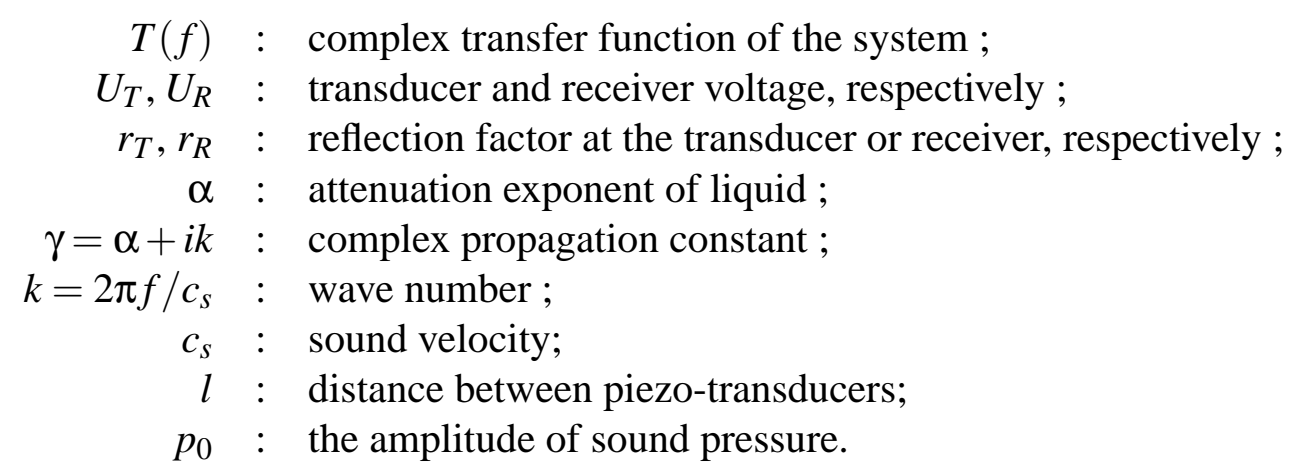

The transfer function for an ideal resonator with reflection coefficients at the liquid transducer interfaces $r_{R}=r_{T}=1$ :

$$
T(f) \propto \frac{1}{e^{\gamma l}-e^{-\gamma l}}=\frac{1}{2 \sinh (\gamma l)} .
$$

With the relation $\sinh ^{2}(\gamma l)=\sinh ^{2}(\alpha l)+\sin ^{2}(k l)$, the amount of $T(f)$ is given by:

$$
|T(f)| \propto \frac{1}{\sqrt{\sinh ^{2}(\alpha l)+\sin ^{2}(k l)}} .
$$

Consequently, for the ideal resonator equidistancy of resonance frequencies follows:

$$
f_{n}=n \cdot \frac{c_{s}}{2 l} \text {. }
$$

With the aid of Eq.(3.47) the sound velocity of $c_{s}$ can be determined. For smaller losses, $(\alpha \ll 1)$ Eq.(3.46) can be calculated with the use of Taylor series, at the resonances $f_{n}$. For $f=f_{n}+\delta f$, the following approximations can be made: $\sin (\alpha l) \approx \alpha l$ and $\sin \left(k_{f_{n}+\delta f} l\right)=\sin \left(\frac{2 \pi\left(f_{n} \delta f\right)}{c_{s}} l\right) \approx \frac{2 \pi \delta f}{c_{s}} l$. Hence, the decrease in the power to onehalf of the original value leads to the relation:

$$
\frac{\left|T\left(f_{n}+\delta f_{h}\right)\right|}{T\left(f_{n}\right)}=\frac{1}{\sqrt{2}}=\frac{\sqrt{(\alpha l)^{2}}}{\sqrt{(\alpha l)^{2}+\left(\frac{2 \pi \delta f_{h}}{c_{s}} l\right)}}
$$

for the sound pressure. Consequently, the relation between the attenuation coefficient $\alpha$ and the half-power bandwidth $\Delta f=2 \delta f_{h}$ follows as:

$$
\alpha=\frac{\pi}{c_{s}} \Leftrightarrow \alpha \lambda=\pi \frac{\Delta f}{f_{n}} .
$$




\section{The real resonator}

\section{- Quality factor the real resonator}

In a real resonator, however, acoustic energy is not only dissipated by the liquid sample but also by imperfections of the cell. Among the various mechanisms is energy dissipation caused by diffraction of the sound wave due to the finite cell diameter. Furthermore, the radiative energy losses at the back face of the quartz, have also to be taken into account. The quality factor $Q$ of a resonator is defined, by the ratio of reversibly stored energy $E_{r}$ and the dissipative energy $E_{d}: Q \equiv 2 \pi E_{r} / E_{d}$. In summary, there are two contributions to the energy dissipation of a sound beam: attenuation caused by the liquid and that from the instruments. The total measured reciprocal quality factor can be written as:

$$
Q_{\text {tot }}^{-1}=Q_{\text {instrum. }}^{-1}+Q_{\text {liquid }}^{-1}
$$

Because the quality factor $Q$ is connected with the half-power bandwidth $\Delta f$ via: $Q=f_{n} / \Delta f$ and because of Eq.(3.50) the total attenuation per wavelength, can be expressed by:

$$
(\alpha \lambda)_{\text {tot }}=(\alpha \lambda)_{\text {instrum. }}+(\alpha \lambda)_{\text {liquid }}
$$

Labhardt et al. [61], [62] have found a relation for losses of plane transducer resonators:

$$
\pi \frac{\Delta f}{\delta}=(\alpha \lambda)_{t o t}=(\alpha \lambda)_{\text {liquid }}+\frac{0.147}{\beta_{b}}\left(\frac{c_{s}}{R}\right)^{3} \cdot \frac{1}{f^{3}}+V_{r}
$$

where $\beta_{b}=Z_{L} / Z_{T}$, with the specific impedances of liquid $Z_{L}$ and transducer $Z_{T}$. The second term on the right-hand side of Eq.(3.52) describes losses caused by diffraction of the sound beam, while $V_{r}$ additional losses. However, it is not possible to analytically separate all instrumental loss contributions during measurements. Owing to this, it is necessary to perform a reference measurement, with a carefully chosen reference liquid with matched sound velocity and density, and to use Eq.(3.50) to calculate $Q_{\text {liquid. }}$.

\section{- Transducer properties:}

The resonances of the transducer can be expressed by the sound velocity of the 
transducer $c_{Q}$ and its thickness $d$ :

$$
f_{Q}=\frac{c_{Q}}{2 d}
$$

There is a finite liquid-to-transducer acoustical impedance ratio so that the sound can penetrate into the transducer. Caused by this effect, the cell-length "seems" to be larger than the geometrical length. This effect becomes more important near the fundamental transducer frequency and its overtones. The cavity resonances are no longer equidistant. This behavior has been calculated by Labhardt, too:

$$
\begin{aligned}
& f_{n}-f_{n-1}=\frac{c_{s}}{2 \pi l} \arccos \left(\frac{\left(g_{n}^{2}-1\right)\left(1-g_{n-1}^{2}\right)-4 g_{n} g_{n-1}}{\left(g_{n}^{2}+1\right)\left(g_{n-1}^{2}+1\right)}\right), n \in \mathbb{N}(3.54) \\
& \text { with } g=\frac{\rho_{\text {liquid }} c_{s}}{\rho_{Q} c_{Q} \tan \left(\pi \frac{f_{n}}{f_{Q}}\right)}
\end{aligned}
$$

with:

$$
\begin{aligned}
f_{n} & : \text { fundamental frequency of the cell; } \\
l & : \text { cell length; } \\
c_{s} & : \text { sound velocity of liquid; } \\
\rho_{\text {liquid }}: & \text { density of liquid; } \\
f_{Q}: & \text { fundamental frequency of the piezo-transducer; } \\
c_{Q}: & \text { sound velocity of the piezo-transducer; } \\
\rho_{Q} & : \text { density of the piezo-transducer. }
\end{aligned}
$$

In order to consider the influence of transducer resonance on the resonator transfer function, a piezo-transducer transfer function $E(f)$ has to be taken into account. Eggers and al. [60] have proposed the function:

$$
E(f)=E_{0}\left[1-i \mathcal{L} \cot \left(\frac{\pi f}{2 f_{T_{m}}}\right)\right]^{-2} .
$$

Here $\mathcal{L}$ is a factor, depending on the acoustic load and $f_{T_{m}}=f_{Q} \cdot m$ with $m=$ $1,2, \ldots, n$.

\section{- Higher order modes:}

At increasing frequency $f$ and increasing attenuation coefficient $\alpha$ of the sample 
liquid, the resonances belonging to a principal mode of vibration of the cavity, will be more and more distorted and disturbed by undesired satellite peaks. The following equation expresses this behavior for the so-called biplanar-resonator:

$$
f_{n}^{m}-f_{n}=\left(\frac{c_{s}}{4 R}\right)^{2}\left(\frac{m(2 m-1)-1}{f_{n}}\right)
$$

where $f_{n}$ denotes the frequency of the $n$-th principal mode, $R$ is the cell radius and $m$ is the number of the higher order satellite modes belonging to $n$ mode ( $m=1$ : principle mode). The distance between the resonance peak of a principle mode and a satellite peak depends on the geometry of the transducer. For the plano-concave resonator, with focussing effect of concavely shaped face of the circular cylindrical cavity resonator, follows:

$$
f_{n}^{m}-f_{n}=m \frac{c}{\pi l} \arccos (\sqrt{1-l / k})
$$

with $k=$ radius of curvature of the concave face. Plano-concave resonator have been also used in this work $(k=2 m)$. A favorable feature of such devices is the reduction of disturbances from mechanical stress during temperature variation, due to the focussing effect.

Other errors typical for measurements are caused by temperature fluctuations (change of $c_{s}$ ), by changes in the geometrical dimensions of cell (due to cleaning, emptying or refilling procedures), errors of electronic equipment, especially of the impedance analyzer used for the transducer function measurements, and systematic errors due to insufficient parallel adjustment of the transducer crystals. In Table (3.1) one can find the instrumental data for relevant resonators of the present work and also some experimental errors.

\section{- Cell design of a resonator:}

The cross section of an ultrasonic resonator cell is shown in Fig.(3.13) on the last but one side of this Chapter. The description can be found in the figure caption.

\section{- Electronic equipment:}

Fig.(3.8) shows a block circuit for broad-band resonator measurements. With the aid of a commercial network analyzer it is possible to measure the transfer function with high accuracy. In addition, a RF pre-amplifier, matching the high-impedance transducer output to the NWA input has been used. Furthermore, applying a computer for control of the network analyzer (NWA) and the amplifier and for the data 


\begin{tabular}{lccccccccc}
\hline \hline geometry & $f_{t}$ & $R_{t}$ & $R$ & $l$ & $f_{c}$ & $V$ & $R_{c}$ & range & error \\
& {$[\mathrm{MHz}]$} & {$[\mathrm{mm}]$} & {$[\mathrm{mm}]$} & {$[\mathrm{mm}]$} & {$[\mathrm{kHz}]$} & {$\left[\mathrm{cm}^{3}\right]$} & {$[\mathrm{m}]$} & {$[\mathrm{MHz}]$} & $\Delta \alpha / \alpha$ \\
\hline $\begin{array}{l}\text { plane-concave } \\
\text { bi-concave }\end{array}$ & 1 & 40 & 35 & 19 & 40 & 75 & 2.0 & $0.1-2.7$ & $5-10 \%$ \\
\hline \hline
\end{tabular}

Table 3.1: Resonator dates: $f_{t}$ : fundamental frequency of the piezo-transducer $; R_{t}:$ transducer radius; $R$ : cell radius; $l:$ cell length; $f_{c}$ : fundamental frequency of the cell; $V$ : liquid volume and $R_{c}$ : radius of curvature of transducer.

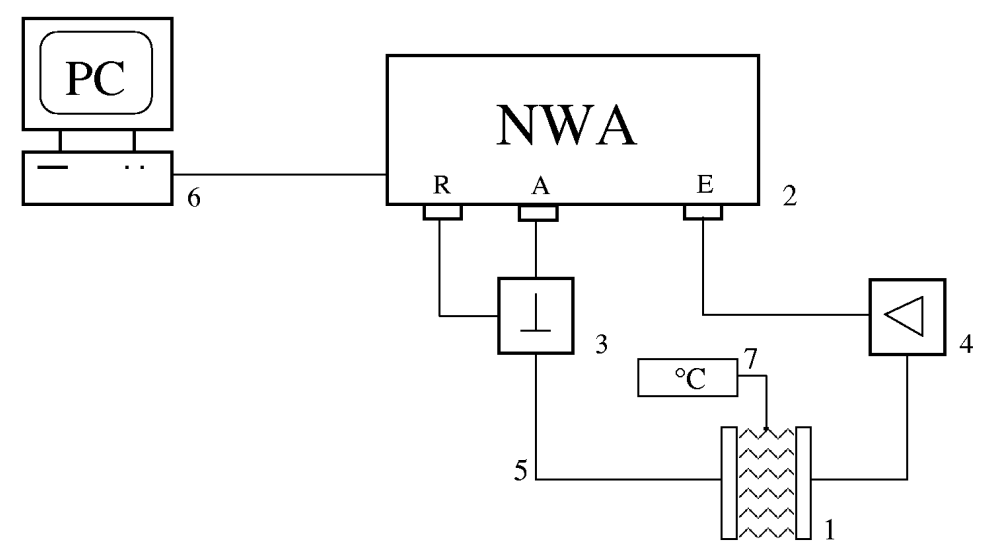

Figure 3.8: Block diagram for measurement of ultrasonic resonator transfer function: (1) sonic cell; (2) network analyzer (NWA) (Hewlett Packard 4195A); (3) signal splitter; (4) pre-amplifier 40dB; (5) coaxial line; (6) computer for process control and data evaluation; (7) Pt-100 thermometer.

evaluation, enabled automatic measurement routines. As presented in Fig.(3.9), the resonator transfer function can not be described by one Lorentz function. Therefore a fitting procedure of the transfer function is performed with the aid of a function, which allows to fit, beside the principal mode, the satellite mode as well as the electrical cross talk:

$$
F_{T}(f)=\left(\sum_{j=1}^{n} \frac{A_{j}}{\sinh \left(\gamma_{j} l\right)}+U e^{i \phi U}\right) e^{i \phi_{0}}
$$

with: 


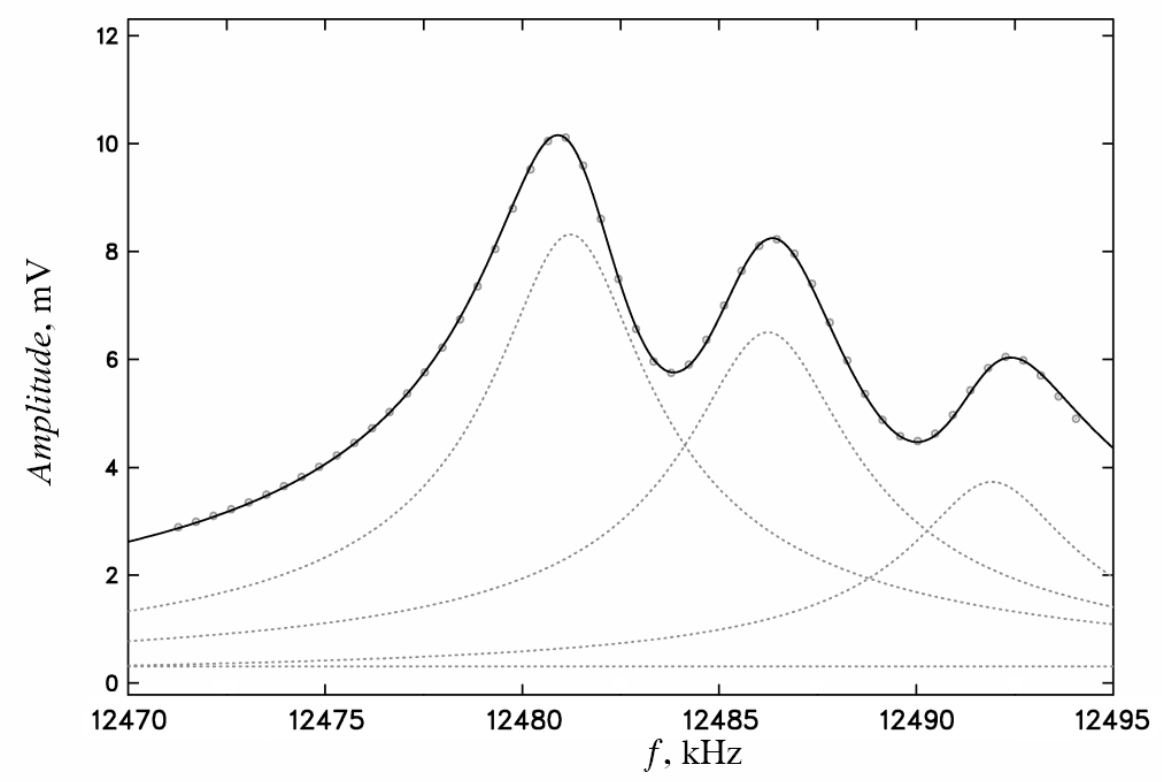

Figure 3.9: Water measurement at resonance frequency of $12 \mathrm{MHz}$, and its satellite modes.

$$
\begin{aligned}
j & : \text { mode number; } \\
\gamma_{j} & =\alpha_{j}+i 2 \pi f / c_{s} \text { (propagation constant); } \\
f_{j} & : \text { resonance frequency } \\
\phi_{0} & : \text { global phase; } \\
l & : \text { cell length; } \\
U & : \text { amplitude of electrical cross talk; } \\
\phi_{U} & : \text { phase of electrical cross talk. }
\end{aligned}
$$

\subsubsection{Pulse-modulated traveling wave methods}

At frequencies above $3 \mathrm{MHz}$ the distortion of resonator curves by higher order modes may be so strong that the separation of the satellite peaks from the main resonance curve becomes impossible. However, the simple proportionality, given by the expression:

$$
p_{R}(x) \sim e^{-\alpha x}
$$

allows to measure quasi-directly the sound attenuation coefficient $\alpha$. The principle of 
pulse-modulated method is shown in Fig.(3.10). Pulse-modulated measurement are performed at the transducer fundamental frequency and its odd overtones which follow the $(2 n+1) \cdot f_{T}$-law. The shiftable receiver, as shown in Fig.(3.10), at the position $X_{1}$ detects the acoustical signal and transfers this into an alternating voltage $U_{R}\left(X_{1}\right)$. At the position $X_{2}$ the alternating voltage $U_{R}\left(X_{2}\right)$ is received. Hence, the attenuation coefficient $\alpha$ follows as:

$$
\alpha=-\frac{\ln \left|U_{R}\left(X_{1}\right)\right|-\ln \left|U_{R}\left(X_{2}\right)\right|}{X_{1}-X_{2}} .
$$

Pulse modulation of the sound wave avoids overlaps between the original signal and

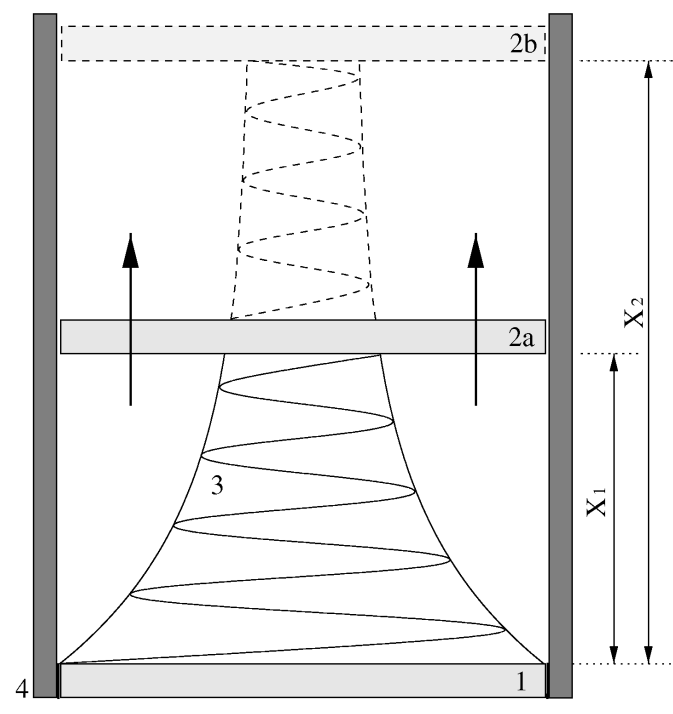

Figure 3.10: Principle of pulse-modulated method: (1) transducer ; (2a) receiver (start position) ; (2b) receiver (end position) ; (3) exponentially decreasing sound wave ; (4) cell walls.

multiply reflected signals as well as the electrical crosstalk. At small transducer spacing, so-called acoustic delay lines (fused quartz) are used for pulse separation. The pressure amplitude $p_{R}$ of the sound wave (taking into account multiple reflections) can be expressed as:

$$
p_{R}(X, t)=p_{T}\left|\frac{(1+r) e^{-\gamma X} e^{i \omega t}}{1-r^{2} e^{-2 \gamma X}}\right|=\frac{p_{T} \cdot(1+r) e^{-\alpha X}}{\sqrt{1+r^{4} e^{-4 \alpha X}+2 r^{2} e^{-2 \alpha X} \cos (2 \beta X)}}
$$

with: 
$p_{T} \quad: \quad$ amplitude of the sound pressure at the transducer;

$p_{R}:$ amplitude of the sound pressure at the receiver;

$X$ : transducer spacing;

$r$ : reflection coefficient;

$\beta=2 \pi / \lambda$ : wave number;

$\gamma=\alpha+i \beta \quad$ : complex propagation constance.

The cosine function of the transfer function Eq.(3.62) describes the so-called $\lambda / 2$-ripple, caused by the multiple reflections of the signal at the transducers. For $\alpha \lambda>3$ the denominator of Eq.(3.62) equals approximately 1, so that an exponential decay of the sound wave $p_{R} \sim e^{-\alpha X}$ follows. In Fig.(3.11) a plot of Eq.(3.62) is given as an example. It illustrates that at decreasing sample length $X$ the standing wave contributions of the ultrasonic field within the cell become more and more important.

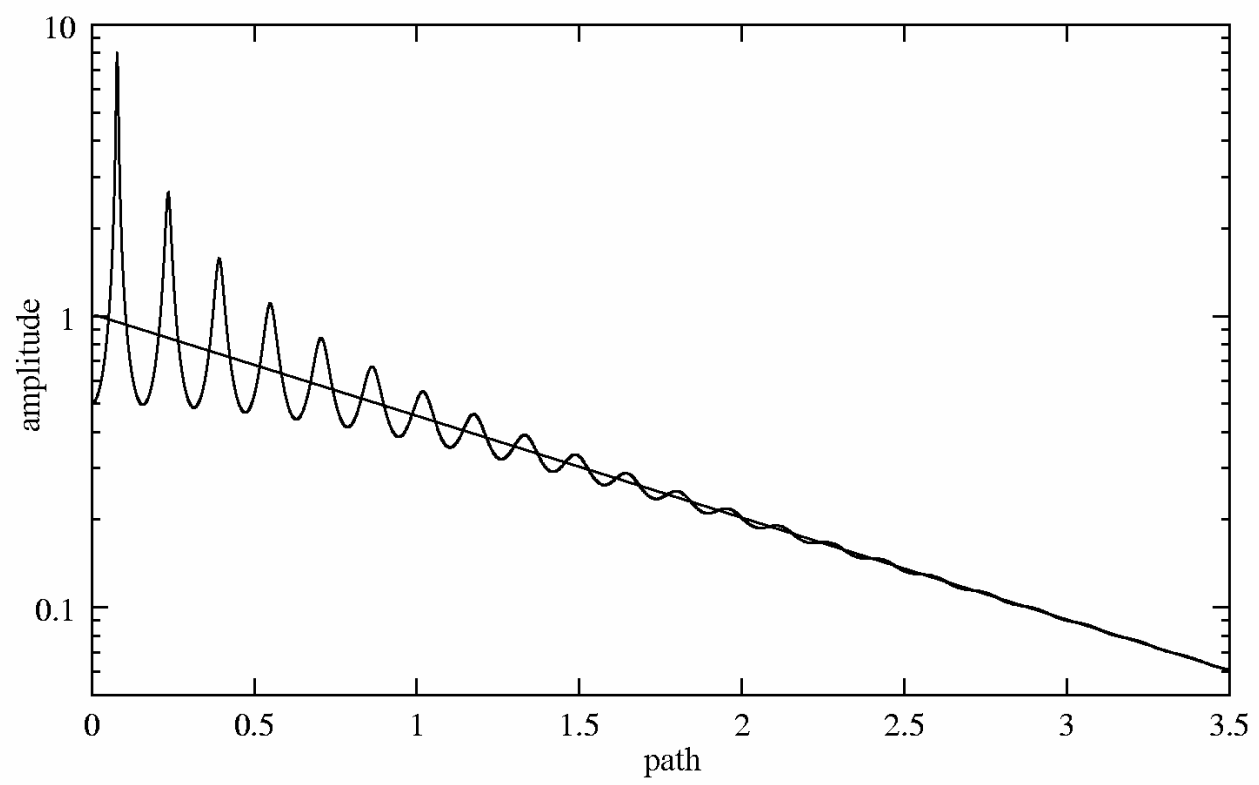

Figure 3.11: Effects of the cosine term in Eq.(3.62) on the transfer function.

Fitting at a given frequency $f$ Eq.(3.62) to experimental data yields the absorption coefficient $\alpha$ and the sound velocity $c_{s}=\lambda f$ of the sample liquid. To raise the accuracy of $\alpha$ measurements it is usual to reduce the influence of the cosine function. This can be realized with the help of the pulse modulation as mentioned before. The transducer signal is pulsed with the aid of square-wave pulse, with the pulse length following the relation: 


$$
\tau<\frac{2 X_{\text {start }}}{c_{s}}
$$

$\tau=2-10 \mu \mathrm{s}$. From this relation it is evident that the knowledge of the sound velocity $c_{s}$ of the liquid sample is essential for correct pulse adjustments.

\section{- Sound velocity measurements:}

The transducer and the receiver have to be brought to a distance fulfilling $X<\tau c / 2$ or $X<3 / \alpha$. At the range of $20 \cdot \lambda$, the transfer function is measured and the experimental data at constant frequency are fitted to Eq.(3.62). Finally, with the help of $c_{s}=\lambda \cdot f$, the sound velocity $c_{s}$ can be calculated.

\section{- Characteristic curve of electronic setup:}

The accuracy of pulse methods depends on the accuracy of determining the characteristic curve of the electronic equipment. In the measurement mode the signal passes some electronic devices. At first, the voltage $U_{R}$ has to be demodulated and amplified. Unfortunately, non-linear effects in the electronic circuit, especially in the amplifier exist. Hence, the voltage $U_{R}$ is subject to the characteristic curve of the receiver $\mathcal{C}\left(U_{R}\right)$ and the sound pressure amplitude at the receiver quartz:

$$
\mathcal{C}\left(U_{R}(X)\right)=\text { const. } \cdot \frac{\left|p_{R}\right|}{\left|p_{T}\right|}
$$

Finally, it is possible, with the aid of linear regression to calculate the attenuation coefficient as given:

$$
\alpha=\left[\ln \left(C\left(U_{R}(X+\Delta X)\right)\right)-\ln \left(C\left(U_{R}(X)\right)\right)\right] / \Delta X .
$$

Here $\Delta X$ is the measurement distance. A central role in the determination of the characteristic curve of the electronic equipment plays the cut-off piston attenuator. Details about that device can be found in [63]. Calibration of the apparatus is performed after each run by switching from the measuring branch to the reference branch and utilizing the cut-off piston attenuator to vary the receiver voltage. The voltage characteristic obtained by this calibration procedure allows the correction of the originally measured $U_{R}$ values.

\section{- Electronic equipment and measuring procedure:}

A block diagram of the electronic apparatus is shown in Fig.(3.12). The full lines 


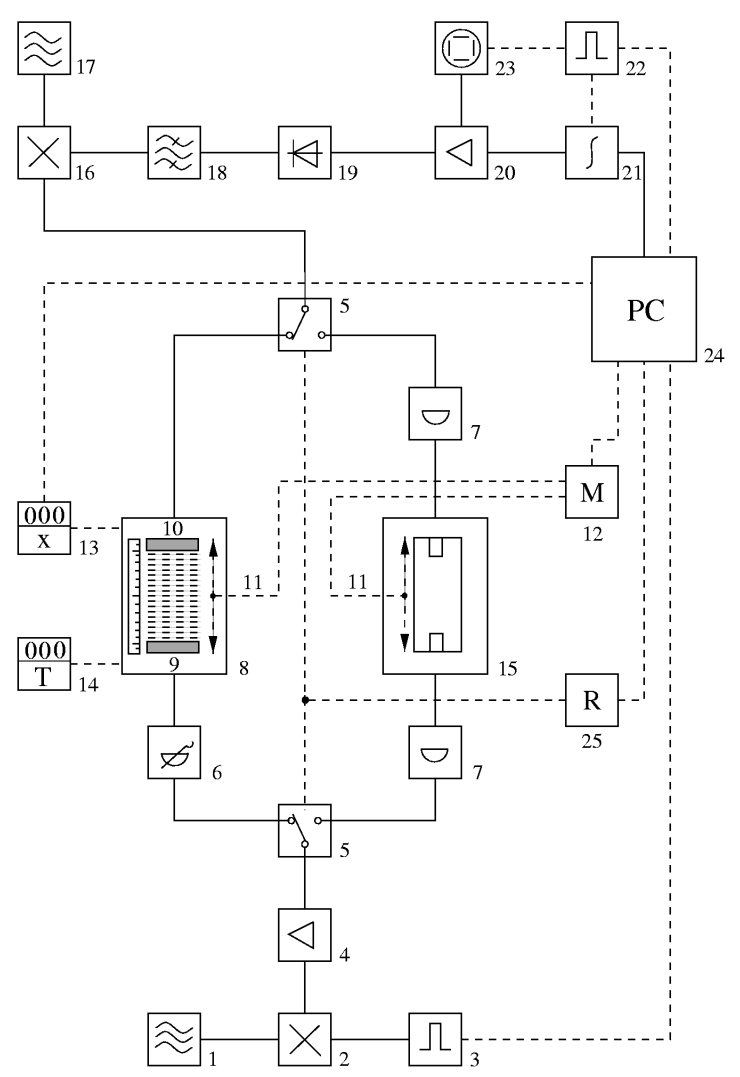

Figure 3.12: Block diagram of the electronic apparatus: (1) frequency synthesizer; (2) mixer; (3) pulse generator; (4) amplifier; (5) HF-change over switch; (6) matching stub transformer; (7) fixed coaxial attenuator; (8) sample cell; (9) transmitter; (10) receiver; (11) stepping-motor; (12) step-motor control; (13) control unit of distance meter (Heidenhain); (14) PT-100 thermometer; (15) cut-off piston attenuator; (16) mixer, (17) oscillator , (18) filter, (19) demodulator (20) amplifier; (21) boxcar integrator and A/D-converter; (22) pulse generator; (23) oscilloscope; (24) personal computer; (25) relays driver card. (the full line indicate the signal path and the dashed lines show the electronic control circuit).

indicate the signal path and the dashed lines show the electronic control circuit. The frequency synthesizer (1) along with the pulse generator (3) and the mixer (2) generates a pulse-modulated HF signal with frequency of measurement $f$. The signal, via two coaxial HF-switches, is passed either through the measuring branch or the reference branch of the comparator circuit. After passing the sample cell or the below-cut-off piston attenuator, both signals are fed via HF change over switch (5) to a superheterodyne receiver (16-20). A boxcar integrator (21) adds up the signal over a sequence of 400 pulses. Finally, the result is transferred to a 
personal computer and evaluated using the data of length $l$, measured by the digital distance meter (11) with control unit (13). The temperature is measured with a Pt-100 thermometer (14).

- Pulse cell parameters: In the present thesis, two kinds of pulse cells have been used to investigate the broadband spectrum of liquids, the 1-MHz- pulse-cell and the 10-MHz-pulse-cell. Some relevant data of the cells are tabulated in Table (3.2). In Fig.(3.14) a cell construction of a 1-MHz-pulse-cell is shown on the last page

\begin{tabular}{ccc}
\hline \hline cell & $\begin{array}{c}1-\mathrm{MHz}- \\
\text { pulse cell }\end{array}$ & $\begin{array}{c}10-\mathrm{MHz}- \\
\text { pulse cell }\end{array}$ \\
\hline transducer & Quarz & LiNbO $_{3}$ \\
$r_{q}[\mathrm{~mm}]$ & 20 & 6 \\
$f_{q}[\mathrm{MHz}]$ & 1.05 & 10.8 \\
$f_{n}$ & $(2 n+1) f_{q}$ & $2 n+1) f_{q}$ \\
$f_{\text {max }}[\mathrm{MHz}]$ & 63 & 530 \\
$\tau[\mu \mathrm{s}]$ & $5-10$ & $2-4$ \\
distance meter & optical, & optical, \\
& MT60 & MT25 \\
position of dist. meter & axial & axial \\
$x_{\text {min }}[\mathrm{nm}]$ & 125 & 125 \\
$x_{\text {max }}[\mathrm{mm}]$ & 40 & 25 \\
$V[\mathrm{ml}]$ & $\approx 130$ & $\approx 10$ \\
\hline \hline
\end{tabular}

Table 3.2: Pulse transmission cells $r_{q}$ : radius and $f_{q}$ : fundamental frequency of transducers; $f_{n}$ : possible measurement frequencies; $f_{\max }$ : maximum frequency; $\tau$ : pulse length; $x_{\text {min }}$ : minimum and $x_{\text {max }}$ : maximum distance between transmitter and receiver; $V$ : sample volume.

of this Chapter. A description can be found in the figure caption. In Table (3.3) experimental errors for measurements with the pulse cells are given.

\subsection{Complementary measurement techniques}

This section deals with additional instruments, which have been used to determine thermodynamic parameters of the investigated liquid systems.

- Sound velocity $c_{s}$ :

For some samples a high resolution ultrasonic velocimeter for measurement of the sound velocity $c_{s}$ has been used. This device consists of two resonator cells, one 


\begin{tabular}{cccc}
\hline \hline cells & frequency range & absorption & error \\
& $f$ & $\alpha / f^{2}$ & $\Delta \alpha / \alpha$ \\
& {$[\mathrm{MHz}]$} & {$\left[10^{-12} \mathrm{~s}^{2} / \mathrm{m}\right]$} & \\
\hline $1-\mathrm{MHz}-$ pulse cell & $13 \leq f \leq 60$ & $\left(\alpha / f^{2}\right) \leq 0.1$ & $3.0 \%$ \\
& $3 \leq f \leq 60$ & $0.1 \leq\left(\alpha / f^{2}\right) \leq 1000$ & $2.0 \%$ \\
\hline & $30 \leq f \leq 50$ & & $2.0 \%$ \\
$10-\mathrm{MHz}-$ pulse cell & $50 \leq f \leq 400$ & & $1.0 \%$ \\
& $400 \leq f \leq 480$ & & $1.5 \%$ \\
\hline \hline
\end{tabular}

Table 3.3: Errors of the pulse transmission cells.

containing the sample and the other one a reference liquid. The fundamental frequency of the transducers is $5 \mathrm{MHz}$. With the knowledge of the sound velocity $c_{1}$ of the reference substance and the cell-length difference $\Delta l$, which normally is negligibly small, between both cells the relation:

$$
c_{2}=2 f_{2} \cdot\left(\frac{c_{1}}{2 f_{1}}-\frac{\Delta l}{n}\right)
$$

is used to determine the sound velocity $c_{2}=c_{s}$ of the sample to within $\Delta c_{s} / c_{s} \approx$ $10^{-5}$. Here $n$ denotes the number $n$ of mode, $f_{1}$ is the resonance frequency in the sample cell and $f_{2}$ in the reference cell.

\section{- Density $\rho:$}

The density $\rho$ was determined with the aid of a high precision vibrating tube densitometer $\left(\Delta \rho / \rho=5 \cdot 10^{-6}\right.$, Physica DMA 5000, Anton Paar, Graz, Austria), with built-in reference oscillator. The relation:

$$
2 \pi f=\sqrt{\frac{k}{m}}=\sqrt{\frac{k}{M_{0}+\rho V}},
$$

where $T=1 / f$ is the period of vibrations with the vibration frequency $f, k$ is the spring constant, $m=M_{0}+\rho V$ the mass, $M_{0}$ the mass of the vibrating tube as well as $V$ the volume of the liquid, allows to determine the density of the liquid under investigation.

\section{- Heat capacity at constant pressure $C_{p}$ :}

Another important parameter is the heat capacity $C_{p}$ at constant pressure. The knowledge of this quantity, allows to calculate directly the critical amplitude $S_{B F}$ of the Bhattacharjee-Ferrell theory, which stands in focus of investigations in present 
work. The heat capacity $C_{p}$ was determined with the aid of a differential scanning calorimeter (MicroCal Inc., Northampton, MA, USA). The device consists of two cells, one containing the sample and the other one the reference liquid. The device measures the excess heating power $\Delta P$ when varying the temperature $T$ with the scan rate $\Delta T / \Delta t$. The excess heat $\Delta Q$ required for the temperature change is directly connected to $\Delta P \cdot \Delta t$ :

$$
\Delta Q=\int_{t}^{t+\Delta t} \Delta P\left(t^{\prime}\right) d t^{\prime} \cong \Delta P \cdot \Delta t
$$

With the aid of the thermodynamic relation $C_{p}=(\partial Q / \partial T)_{p}$ it is possible to determine the heat capacity at constant pressure.

\section{- Shear viscosity $\eta_{s}$ :}

Static shear viscosity measurements of the critical systems have been performed with a set of Ubbelohde-type capillary viscosimeters (Schott, Germany) or, in order to avoid the risk of change in the the mixture composition due to preferential evaporation, with a falling ball viscosimeter (Haake, Karlsruhe, Germany). With some samples $^{4}$, a shear wave impedance spectrometer, operated between $5 \mathrm{MHz}$ and 130 $\mathrm{MHz}$, has been additionally used. The principle of measurement consists in the determination of the shift in the series of resonance frequency $f_{n}$ and of a change in the quality factor resulting from loading a shear quartz with the liquid. The quartz as the central part of a shear resonator [64] is a carefully cut planar AT-quartz disc, beveled at its back. If the shearing stress oscillates so rapidly that its time period is shorter that the time required for the molecules in the sample to adopt their relative position, a dispersion in the shear viscosity results. The dispersion and the phase lag are taken into account by using a frequency-dependent complex shear viscosity:

$$
\eta_{s}(f)=\eta_{s}^{\prime}(f)-i \eta_{s}^{\prime \prime}(f)
$$

Here $\eta_{s}^{\prime}(f)$ represents the irreversible viscous molecular processes and the imaginary part $i \eta_{s}^{\prime \prime}(f)$ represents the reversible elastic processes.

\footnotetext{
${ }^{4}$ critical mixtures: 2,6-dimethylpyridine-water and triethylamine-water
} 


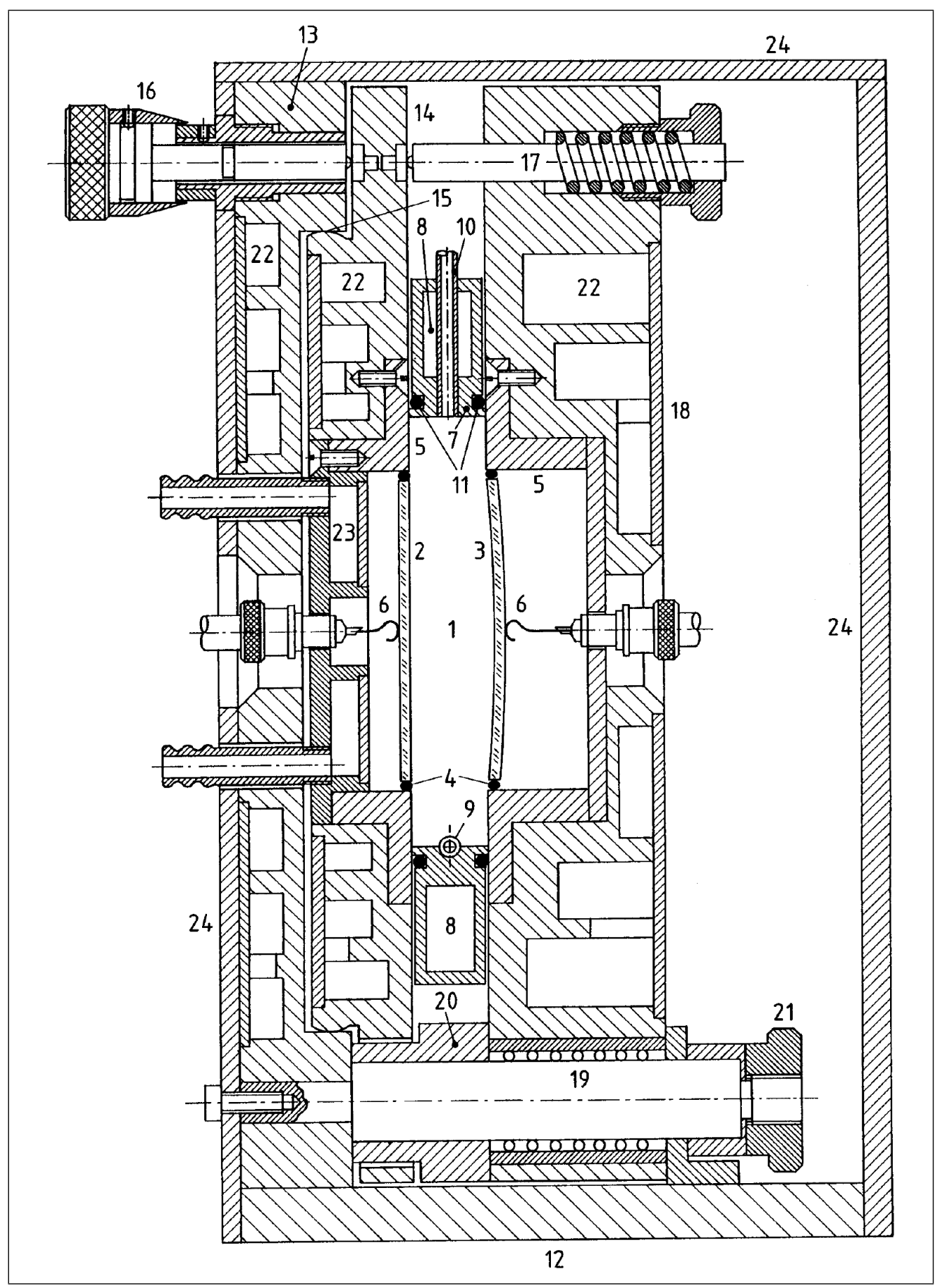

Figure 3.13: Cross section of a plano-concave resonator (1) sample cavity; (2) plane piezo-electric transducer; (3) concave piezo-electric transducer; (4) layer of silicone rubber with embedded electrical wires to ground of front side of transducer ; (5) transducer fixture (stainless-steel); (6) flexible electric contact wire; (7) cell jacket; (8), (22), (23) channels for circulating thermostat fluid ; (9), (10) inlet and outlet; (11) sealing O-ring; (12) main frame; (13) frame at adjustable transducer side; (14) adjustable plate ; (15) ball-and-socket joint; (16) screw for adjustment of (14); (17) spring ; (18) mounting plate; (19) ball bush guides; (20) precision-gauge block establishing the distance to; (21) locking device; (24) thermostatic coat. 


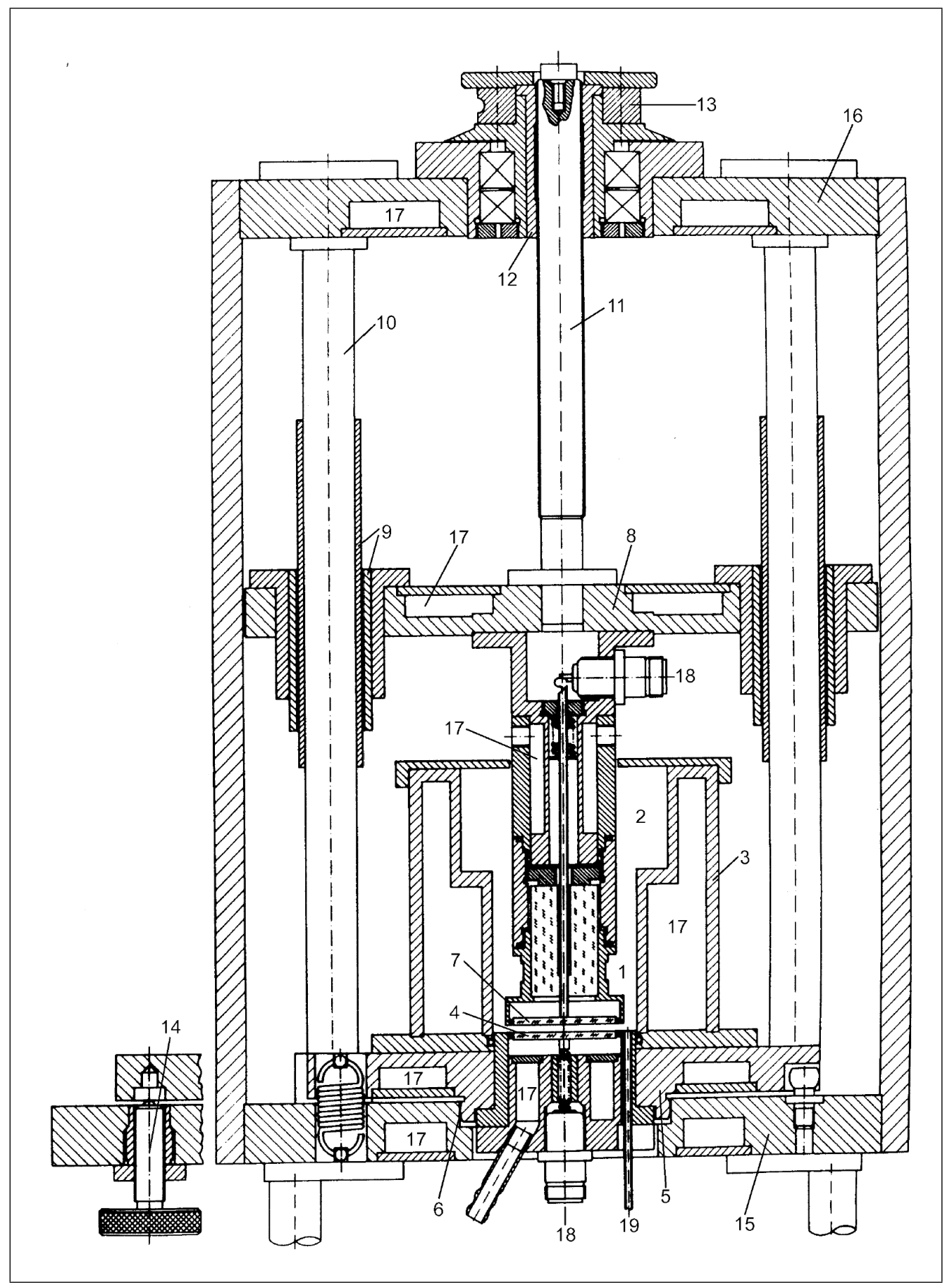

Figure 3.14: The cross section of a 1 MHz-pulse-cell (1) sample volume and (2) sample reservoir; (3) thermostatic walls ; (4) transducer; (5) tiltable mounting plate; (6) ball-andsocket joint; (7) receiver; (8) movable mounting plate; (9) ball bush guides; (10) lapped pin; (11) spindle; (12) nut; (13) ratched wheel ; (14) adjustment screws for transducer parallelism; (15) base plate; (16) mounting plate ; (17) channels for circulating thermostat fluid; (18) $\mathrm{N}$ connector; (19) outlet. 


\section{Critical Contribution, Dynamic Scaling and Crossover Theory}

The following Chapter deals with the dynamic scaling aspects within the framework of Bhattacharjee-Ferrell theory. Furthermore, relationships between the critical sound attenuation and the dynamic scaling function are presented. Moreover, crossover effects for binary and ternary fluids are presented.

\subsection{Bhattacharjee-Ferrell scaling hypothesis - binary systems}

Critical phenomena, as all continuous phase transitions, Show universal characteristics of their thermodynamic properties, if they belong to the same universality class and if their dimension is identical. In Section (2.3.3) the concepts and consequences of critical slowing down have been presented. In particular, the light scattering is well represented and described by dynamic scaling theories, resulting from the mode-coupling considerations. However, the treatment of critical ultrasonic attenuation necessitates the development of new theories in order to get an access to critical fluctuations in a sound field. Bhattacharjee and Ferrell have presented [18], [19] a general theory of the critical ultrasonic attenuation, based on an extension of the concept of the frequency-dependent specific heat. This conception was firstly introduced by Herzfeld and Rice [65] in 1928.

\subsection{Critical sound attenuation}

For understanding the nature of critical sound attenuation, it is important to study the propagation velocity $c_{s}$ of low-frequency sound in the vicinity of the critical point [20]. This kind of considerations allows to understand the coupling between the sound propagation and sound attenuation as well as the sound dispersion of sound velocity near the consolute point. The first thermodynamic studies of Bhattacharjee and Ferrell concentrated on the variation of volume $V$ with pressure $p$, due to sound propagation at constant entropy $S$. As consequence of this examinations they got the isentropic compressibility $\beta_{S}$ at constant $S$ : 


$$
\beta_{S}=\beta_{S, c}+\frac{S_{c}^{\prime}}{V_{c}}\left(\frac{\partial \Delta T}{\partial p}\right)_{S}
$$

where $\Delta T=T-T_{c}(p)$ and index $c$ denotes critical parameters. Moreover, the adiabatic temperature variation $(\partial \Delta T / \partial p)_{S}$ in Eq.(4.1), expressed by:

$$
\left(\frac{\partial \Delta T}{\partial p}\right)_{S}=-\frac{(\partial S / \partial p)_{\Delta T}}{\partial S / \partial \Delta T)_{p}}=-\simeq \frac{T_{c} S_{c}^{\prime}}{C_{p}}=-g \frac{V_{c}}{C_{p}}
$$

produces a dimensionless parameter $g$, which represents the system-specific coupling constant and describes the magnitude of coupling between critical density fluctuations and the propagating sound wave:

$$
g=\frac{T_{c} S_{c}^{\prime}}{V_{c}}
$$

Substitution of Eq.(4.2) and Eq.(4.3) into Eq.(4.1) yields a relationship which is accessible to experiments:

$$
\beta_{S}=\beta_{S, c}-\frac{V_{c}}{T_{c}} \frac{g^{2}}{C_{p}}
$$

The sound velocity can be expressed as:

$$
c_{s}^{-2}=c_{s, c}^{-2}-\frac{g^{2}}{T_{c} C_{p}},
$$

where $c_{s, c}$ denotes the sound velocity at the critical point. Approximately one can describe the sound velocity $c_{s}$ by the following relation:

$$
c_{s}=c_{s, c}+\frac{g^{2} c_{s, c}^{3}}{2 T_{c} C_{p}} .
$$

The next step in the procedure of Bhattacharjee and Ferrell considers the frequency $\omega$ for an applied pressure signal with its time dependence given by $\exp (-i \omega t)$. Furthermore, with the aid of the frequency-dependent specific heat $\tilde{C}_{p}(\omega)$ it is possible to express the sound velocity $c_{S}$ in Eq.(4.6) and the compressibility $\beta_{S}$ in Eq.(4.4) as frequencydependent quantities ${ }^{1}$ :

\footnotetext{
${ }^{1}$ tilde denotes complex quantities, except for critical exponents
} 


$$
\tilde{c_{s}}=c_{s, c}+\frac{g^{2} c_{s, c}^{3}}{2 T_{c} \tilde{C}_{p}(\omega)}
$$

and

$$
\tilde{\beta}_{S}=\beta_{S, c}-\frac{V_{c}}{T_{c}} \frac{g^{2}}{\tilde{C}_{p}} .
$$

It is assumed that the critical part of the specific heat determines the complex sound velocity in Eq.(4.7). Within the scope of dynamic scaling it is possible to write the dependence of $C_{p}$ on the reduced temperature $\varepsilon$ :

$$
C_{p}(\omega=0) \propto \varepsilon^{-\tilde{\alpha_{0}}},
$$

where $\tilde{\alpha_{0}}$ denotes the critical exponent. With the aid of the relationship of critical diffusion coefficient in [66], [22], that controls the relaxation of the concentration fluctuations and relates the diffusion coefficient $D$, to the viscosity $\eta_{s}$ and the correlation length $\xi$ of critical fluctuations:

$$
D=\frac{k_{B} T}{6 \pi \eta_{s} \xi}
$$

(with the Boltzmanns's constant $k_{B}$, and absolute temperature $T$ ), it is possible to give an expression for the characteristic relaxation rate of a fluid:

$$
\Gamma(\varepsilon)=\frac{2 D}{\xi^{2}}=\Gamma_{0} \varepsilon^{Z_{0} \tilde{v}}
$$

where $\tilde{v}$ denotes the critical exponent of the correlation length and $Z_{0}$ denotes the dynamic critical exponent. The amplitude $\Gamma_{0}$ is a characteristic system-dependent constant. The system dependent values $\Gamma_{0}$ of several binary systems are presented in Table (5.10, s.121). However, with regard to ultrasonic spectroscopy, it might be interesting to investigate the frequency dependence of the specific heat $C_{p}$. Using Eq.(4.11) one can express the temperature dependence in $C_{p}$ in terms of the characteristic relaxation rate $\Gamma$ :

$$
C_{p}(\omega=0) \sim \varepsilon^{-\tilde{\alpha_{0}}} \Rightarrow C_{p}(\Gamma, \omega=0) \sim \Gamma^{\frac{-\tilde{\alpha_{0}}}{\bar{Z}_{0} \tilde{v}}},
$$

where the value of the exponent is:

$$
\frac{\tilde{\alpha}_{0}}{Z_{0} \tilde{v}}=\frac{0.11}{3.05 \times 0.63}=0.057
$$


Consequently, one can get the frequency dependence of $C_{p}$ at $\Gamma=0$, by using the modecoupling formalism. It follows:

$$
\tilde{C_{p}}(\Gamma=0, \omega) \propto\left(\frac{-i \omega}{a}\right)^{-\tilde{\alpha_{0}} / Z_{0} \tilde{v}},
$$

with a constant $a$. The attenuation coefficient $\alpha$ introduced in Chapter (3), is proportional to:

$$
\begin{aligned}
\alpha \sim \omega \operatorname{Im} \tilde{C_{p}}(\omega)^{-1} & \simeq \frac{-\omega}{\left(\operatorname{Re} \tilde{C_{p}}\right)^{2}} \operatorname{Im} C(0, \omega) \\
& =\frac{-\omega^{2}}{a\left(\operatorname{Re} \tilde{C_{p}}\right)^{2}}\left(\frac{a}{\omega}\right)^{1+\tilde{\alpha_{0}} / Z_{0} \tilde{v}} \sin \left(\frac{-\tilde{\alpha_{0}} \pi}{2 Z_{0} v}\right) \\
& \simeq \frac{\tilde{\alpha_{0}} \pi}{2 Z_{0} \tilde{v} a} \frac{\omega^{2}}{\left(\operatorname{Re} \tilde{C_{p}}\right)^{2}}\left(\frac{a}{\omega}\right)^{1+\tilde{\alpha_{0} / Z_{0} \tilde{v}}} \\
\Rightarrow\left(\frac{\alpha}{f^{2}}\right)_{c} & \sim f^{-1-\tilde{\alpha_{0}} / Z_{0} \tilde{v}}=f^{-1.057} .
\end{aligned}
$$

The plot of $\alpha / f^{2}$ versus $f^{-1.057}$ represents a straight line as is shown by the example of the $n$-pentanol-nitromethane mixture of critical composition in Fig.(4.1).

Up to now, the temperature dependence of the amplitude has not be considered. Bhattacharjee and Ferrell give an expression for the amplitude of critical contribution:

$$
R_{c}(f)=(\alpha \lambda)_{c}=A(T) \cdot F_{B F}(\Omega) \text { with } A(T)=c_{s} \cdot S_{B F} \cdot \Gamma^{-\tilde{\alpha_{0}} / Z_{0} \tilde{v}}
$$

with:

$$
\begin{array}{rll}
S_{B F} & : & \text { the temperature dependent Bhattacharjee-Ferrell amplitude } \\
F_{B F} & : & \text { the Bhattacharjee-Ferrell scaling function } \\
\Gamma & : & \text { relaxation rate of concentration fluctuations } \\
\Omega=\omega / \Gamma & : & \text { reduced frequency } \\
c_{S} & : & \text { sound velocity. }
\end{array}
$$

According to Eq.(4.16), where $\tilde{\alpha_{0}} / Z_{0} \tilde{\mathrm{v}}=0.057$, the amplitude parameter $A$ is related to 


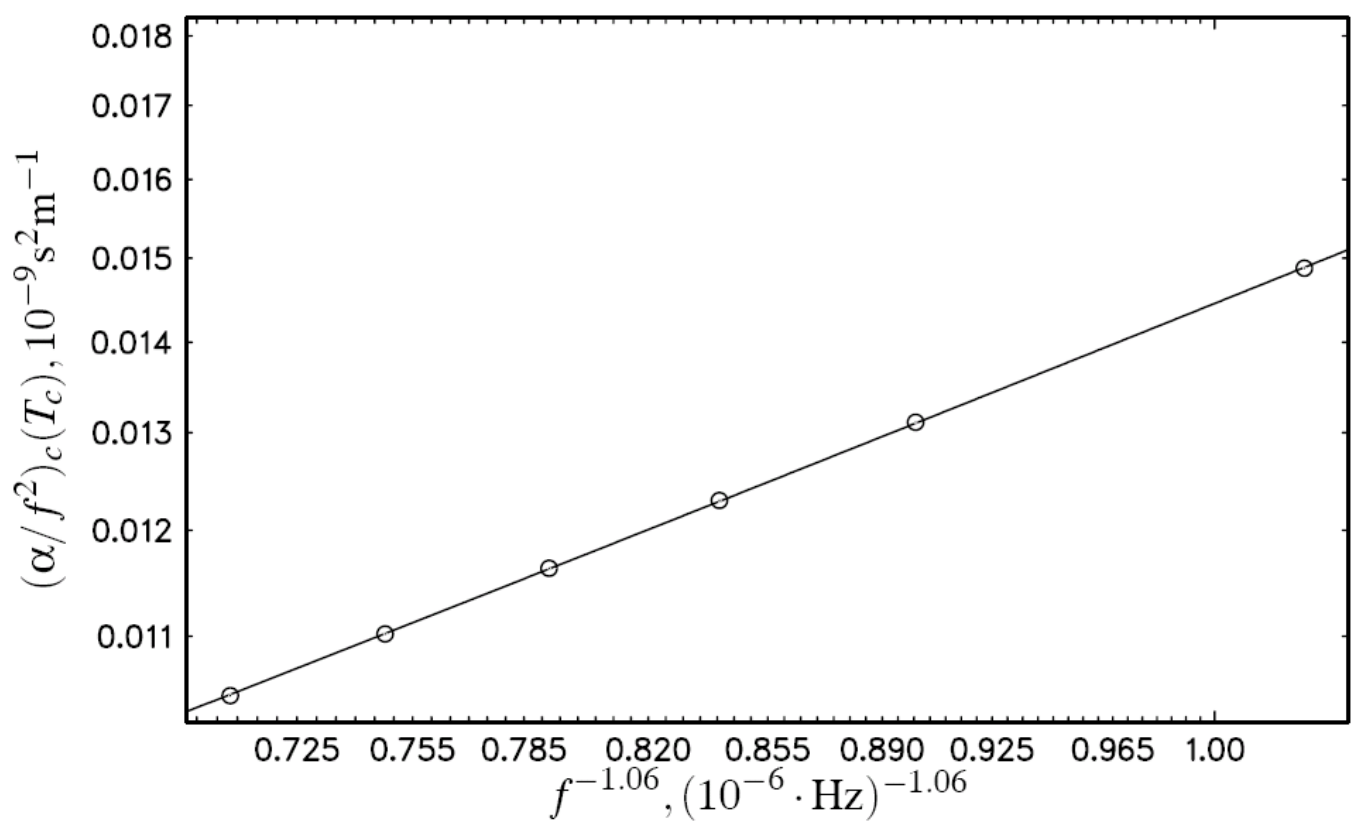

Figure 4.1: Critical part in the frequency-normalized attenuation coefficient for the npentanol-nitromethane mixture: at $T_{c}$ plotted as function of $f^{-1.06}[76]$.

the amplitude of the Bhattacharjee-Ferrell dynamic scaling model:

$$
S_{B F}=\frac{\pi^{2} \delta \Delta C_{p} c_{s}}{2 C_{p b}^{2} T_{c}}\left[\frac{\Omega_{1 / 2}^{B F} \Gamma_{0}}{2 \pi}\right]^{\delta} g^{2}
$$

of the dynamic scaling model. Parameter $g$ in Eq.(4.17) is the adiabatic coupling constant defined by Eq.(4.3). According to the thermodynamic relation:

$$
g=\rho C_{p}\left(\frac{\mathrm{d} T_{c}}{\mathrm{~d} p}-\frac{T_{c} \alpha_{p}}{\rho C_{p}}\right)
$$

it can be obtained from the slope $d T_{c} / d p$ in the pressure dependence of the critical temperature along the critical line and to the thermal expansion coefficient $\alpha_{p}$ at constant pressure. The latter can be expressed with the aid of density $\rho$ likewise at constant pressure:

$$
\alpha_{p}=\rho\left[\mathrm{d} \rho^{-1} / \mathrm{d} T\right]_{p}
$$

In Eq.(4.17) $\Delta C_{p}$ as well as $C_{p b}$ are the amplitudes of the critical part and the background part, respectively, of the heat capacity at constant pressure:

$$
C_{p}(\varepsilon)=\Delta C_{p} \varepsilon^{-\tilde{\alpha_{0}}}+C_{p b}
$$


The function $F_{B F}(\Omega)$ in Eq.(4.16), is the so-called scaling function, which plays a central role it the Bhattacharjee-Ferrell theory. The properties of this special function will be treated with more details in the next section.

\subsection{The scaling function $F_{x}(\Omega)$}

Last decades, various theoretical expressions for the universal scaling function of ultrasonic attenuation spectra have been presented for critically demixing binary fluids. In Fig.(4.3) three prominent examples are shown.

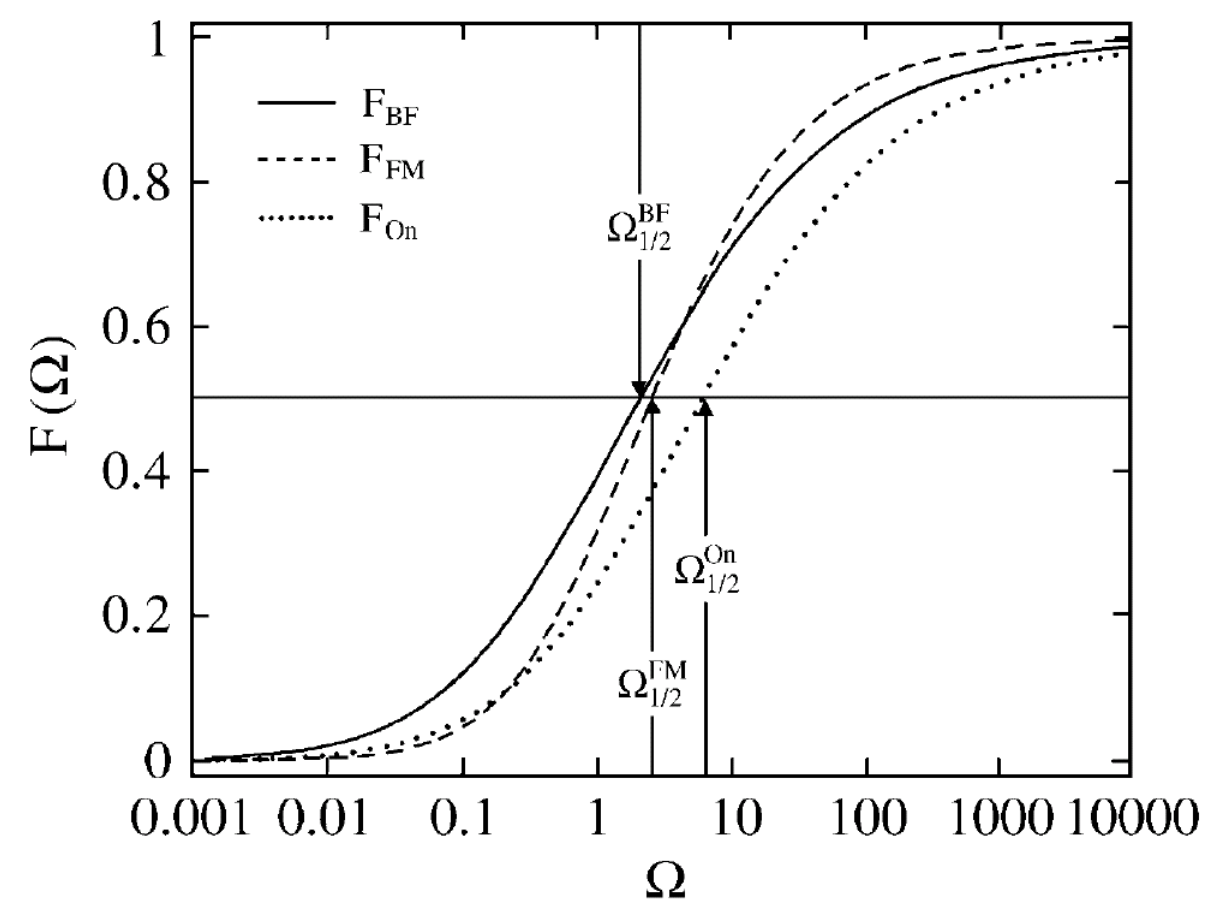

Figure 4.2: Three prominent examples of scaling functions: the full line represents the Bhattacharjee-Ferrell (BF), the dashed line the Folk-Moser (FM), and the dotted line the Onuki (On) function [67].

In 1981 Bhattacharjee and Ferrell have presented the scaling function for sonic attenuation in analytical form [18]:

$$
F(\Omega)=\frac{3}{\pi} \int_{0}^{\infty} \frac{x \cdot d x}{\left(1+x^{2}\right)^{2}} \cdot \frac{\Omega \cdot x \cdot(1+x)^{1 / 2}}{x^{2} \cdot(1+x)+\Omega^{2}} .
$$

Because of the difficulties to treat this integral, an empirical function has been developed 
later in [20]:

$$
F_{B F}(\Omega)=\frac{1}{\left[1+0,4142\left(\frac{\Omega_{1 / 2}}{\Omega}\right)^{1 / 2}\right]^{2}}
$$

with the half attenuation frequency $\Omega_{1 / 2}$. The Folk-Moser (FM) scaling function $F_{B F}(\Omega)$ as resulting from the renormalization group theory of the mode-coupling model [23], [24], and the function $F_{O n}(\Omega)$, which Onuki (On) derived from an intuitive description of the bulk viscosity near a consolute point, published in [25] and [26], are not available from theory in analytical form. Therefore, Behrends at al. developed a quasi-universal empirical form of scaling functions [67], in correspondence with the empirical function of Bhattacharjee and Ferrell. These forms are shown in Fig.(4.3), and described by following relation:

$$
F_{x}(\Omega)=\left[1+\left(\frac{\Omega_{x}}{\Omega}\right)^{n_{x}}\right]^{-2}=\left[1+0.4142\left(\frac{\Omega_{1 / 2}^{x}}{\Omega}\right)^{n_{x}}\right]^{-2}
$$

where $x$ denotes $B F, F M$ and $O n$, while $\Omega_{x}$ and $\Omega_{1 / 2}^{x}$ denote a characteristic frequency and the half-attenuation frequency of the scaling function, respectively. The crucial parameter in Eq.(4.23) is the exponent $n_{x}$, which is related to the logarithmic slope $S_{x}\left(\Omega=\Omega_{1 / 2}\right)=$ $\mathrm{d} F_{x}(\Omega) /\left.\mathrm{d} \ln (\Omega)\right|_{\Omega_{1 / 2}}$, that dominates the shape of the scaling functions. In [67] the following parameter values are given: BF, $\Omega_{1 / 2}=2.1, n_{x}=0.500, S_{x}=0.146 ; \mathrm{FM}, \Omega_{1 / 2}=$ $3.1(1), n_{x}=0.635(5), S_{x}=0.186$; On, $\Omega_{1 / 2}=6.2(1), n_{x}=0.500(2), S_{x}=0.146$;). Finally, using the relation

$$
\Omega_{1 / 2}^{x}=\frac{2 \pi f}{\Gamma(\varepsilon)}\left[\frac{1}{0.4142}\left(\left(\frac{(\alpha \lambda)_{c}\left(T_{c}\right)}{(\alpha \lambda)_{c}(T)}\right)^{1 / 2}-1\right)\right]^{1 / n_{x}}
$$

to evaluate experimental attenuation coefficient data along with relaxation rates $\Gamma(\varepsilon)$ from light scattering and shear viscosity, it is possible to decide about the quality and validity of the scaling functions.

The determination of the scaling function $F_{x}(\Omega)$ is usually based on the fact that $A$ and $c_{s}$ in Eq.(4.16) are only weakly dependent upon temperature. Therefore, the scaling function can be derived as the ratio:

$$
F_{x}(\Omega)=(\alpha \lambda)_{c}(f, T) /(\alpha \lambda)_{c}\left(f, T_{c}\right), F_{x}(\Omega)=1 \text { for } T \rightarrow T_{c}
$$

The measured total attenuation data contain contribution from critical fluctuation but also 
from noncritical processes. Assuming that all parts contribute to the ultrasonic attenuation spectrum additively, the ultrasonic spectra can be analytically represented as a sum (shown in Section (3.3.1.1) of $N$ Debye terms, the background contribution and the critical contribution:

$$
\left(\frac{\alpha}{f^{2}}\right)=\underbrace{S_{B F} f^{-(1+\delta)} \cdot F_{B F}}_{R_{c}^{+}(f)}+\underbrace{\sum_{n=1}^{N} \frac{A_{D_{n}}}{1+\omega^{2} \tau_{D_{n}}^{2}}}_{R_{D_{n}}^{+}(f)}+\frac{B}{c_{s}} .
$$

The Debye-terms ${ }^{2}$ can be likewise replaced by Hill-terms as has been shown also in Section (3.3.1.1).

\subsection{The crossover theory in binary mixtures}

\section{General conceptions of crossover theory}

The classical theories by van der Waals, Bertholt, and Dieterici describe pretty well the hydrodynamic and thermodynamic behavior of classical fluids in the mean-field region. Moreover, all their classical equations show the existence of a critical point. Unfortunately, they do not predict the non-analytic behavior in real systems. This fluid domain has been treated by Wilson, Fisher and Wagner within the framework of the renormalizationgroup theory. The conceptions and formalisms of this theory lead to a description in terms of scaling laws near the consolute point. Furthermore, renormalization-group theory has been quite successful in calculations and predictions of critical exponents. However, due to crossover effects this theory has a limited range of validity.

$$
\text { Critical Region } \leftarrow \text { Crossover Region } \leftarrow \text { Mean-Field }
$$

Unfortunately, the theoretical descriptions are valid only in a range extremely close to the critical point when $\varepsilon \rightarrow 0$. There is no conception of extrapolation from the mean-field to the critical region. This so-called crossover-range has been treated 1986 in a paper by Albright at al [68], consistent with the renormalization-group theory. Their crossover descriptions for properties of fluids take into account, that besides the contributions from critical fluctuations to the critical behavior, there are further degrees of freedom, such as changes of molecular conformations and of the extent of hydrogen bonding. These kinds of effects do not couple to the critical fluctuations. Therefore, it is adequate to divide the

\footnotetext{
${ }^{2}$ note: $(+)$ in $R_{c}^{+}(f)$ or $R_{D}^{+}(f)$ refers to the form of presentation of ultrasonic spectra (attenuation-perwavelength $(\alpha \lambda)$ or $\left(\alpha / f^{2}\right): R_{D}(f) \cdot \frac{f}{c_{s}}=R_{D}^{+}(f)$
} 
modes of the considered liquid system into those that show only short range order and high frequency fluctuations and are only weakly coupled and into such which show long range fluctuations and are coupled strongly. These last ones lead to non-analytic behavior near the consolute point. Consequently, the existence of a cut-off $\Lambda$ in the wave numbers of fluctuations, has to be taken into account when fluctuation dominated behavior of the system is studied. This view has been successfully applied to the van der Waals gas.

\section{The Crossover corrections}

In the case of the dynamic light scattering, the shear viscosity as well as ultrasonic attenuation spectroscopy crossover corrections have to be taken into account when $T$ is not sufficiently close to $T_{c}$. It has been shown in [16], [69] and [17], that close to the critical point, in the asymptotic limit, the shear viscosity can be described by the expression:

$$
\eta_{s}(\varepsilon)=\eta_{b g}\left(Q_{0} \xi\right)^{Z_{\eta}}
$$

here $Q_{0}$ denotes the system-dependent critical amplitude and $\xi$ the fluctuation correlation length. The background viscosity $\eta_{b g}$ is given by the relation:

$$
\eta_{b g}(\varepsilon)=A_{\eta} \exp \left[B_{\eta} /\left(T-T_{\eta}\right)\right] \text { or } \eta_{b g}(\varepsilon)=A_{\eta} \exp \left[B_{\eta} / T\right]
$$

with the system specific parameters $A_{\eta}, B_{\eta}$, and $T_{\eta}$ and with the absolute temperature $T$. The inverse critical amplitude of the viscosity $Q_{0}^{-1}$, can be written as:

$$
\frac{1}{Q_{0}}=\frac{e^{4 / 3}}{2}\left(\frac{1}{q_{D}}+\frac{1}{q_{c}}\right),
$$

where $q_{c}$ and $q_{D}$ are the noncritical cut-off wave numbers. Eq.(4.28) is correct only in a region close to the critical point. Therefore, when treating data over a large temperature range, it is essential to consider the crossover corrections as has been presented by Burstyn at al. [16]. In that paper Burstyn et al. introduce a crossover function $H\left(\xi, q_{c}, q_{D}\right)$, which is also dependent on the noncritical cut-off wave numbers $q_{c}, q_{D}$ as well as the correlation length $\xi$ :

$$
\eta_{s}(\varepsilon)=\eta_{b g}(T) \exp \left(Z_{\eta} H\left(\xi(\varepsilon), q_{D}, q_{c}\right)\right)
$$

with

$$
H=\frac{1}{12} \cdot \sin \left(3 \cdot \Psi_{D}\right)-\frac{1}{4 q_{D}} \cdot \xi \cdot \sin \left(2 \cdot \Psi_{D}\right)
$$




$$
\begin{aligned}
& +\frac{1}{\left(q_{c} \xi\right)^{2}} \cdot\left[1-\frac{5}{4}\left(q_{c} \xi\right)^{2} \sin \left(\Psi_{D}\right)\right] \\
& -\frac{1}{\left(q_{c} \xi\right)^{3}} \cdot\left\{\left[1-\frac{3}{2}\left(q_{c} \xi\right)^{2}\right] \cdot \Psi_{D}-\left|\left(q_{c} \xi\right)^{2}-1\right|^{3 / 2} \cdot L(\tilde{\omega})\right\}
\end{aligned}
$$

where

$$
\begin{aligned}
\Psi_{D} & =\arccos \left(1+q_{D}^{2} \cdot \xi^{2}\right)^{-1 / 2} \\
\tilde{\omega} & =\left|\frac{q_{c} \xi-1}{q_{c} \xi+1}\right|^{1 / 2} \tan \left(\Psi_{D} / 2\right) \\
L(\tilde{\omega}) & =\ln \left(\frac{1+\tilde{\omega}}{1-\tilde{\omega}}\right) \text { if } q_{c} \xi>1 \\
& =2 \cdot \arctan |\tilde{\omega}| \text { if } q_{c} \xi \leq 1
\end{aligned}
$$

For large $\varepsilon$, the crossover function defined by Eq.(4.31) behaves as $H\left(\xi(\varepsilon), q_{D}, q_{c}\right) \rightarrow 0$, so that $\eta \rightarrow \eta_{b g}$. In the asymptotic limit the Eq.(4.31) simplifies to the power law in Eq.(4.28). The influence of the crossover function is not only restricted to the shear viscosity. The cut-off wave numbers $q_{c}$ and $q_{D}$ play also an important role in the mutual diffusion coefficient, which is given by:

$$
D=\Delta D+D_{b g}
$$

The value $\Delta D$ represents the singular contribution, which is shaped by the Kawasaki function $\Omega_{K}$ [66]:

$$
\Omega_{K}(x)=\frac{3}{4 x}\left[1+x^{2}+\left(x^{3}-\frac{1}{x}\right) \arctan x\right]
$$

with $x=q \xi$. The mutual diffusion is then represented by:

$$
D=\underbrace{1,03 \cdot \frac{k_{B} \cdot T}{6 \pi \eta_{s} \xi} \cdot \Omega_{K}(x)\left[1+\left(\frac{x}{2}\right)^{2}\right]^{z_{\eta} / 2}}_{\Delta D}+\underbrace{\frac{k_{B} \cdot T}{16 \eta_{b} \cdot \xi} \cdot\left(\frac{1+q^{2} \xi^{2}}{\tilde{q_{c}} \cdot \xi}\right)}_{D_{b g}}
$$

with: 


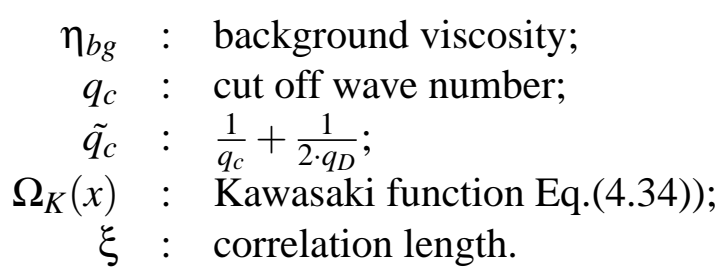

Another important consequence resulting from crossover corrections is the significant influence of the values $\xi_{0}$ and $q_{c}$ on the reduced temperature $\varepsilon$. Bhattacharjee and Ferrell have presented a correction expression that is based on the use of an effective reduced temperature $\widetilde{\varepsilon}$, which is given by:

$$
\widetilde{\varepsilon}=\varepsilon\left[1+\beta \frac{1}{\xi q_{c}}\right]^{1 / 2},
$$

with the parameter $\beta=1.18$. The significance of the cut-off wave number $q_{c}$ has been demonstrated in [70]; due to a large value of $q_{c}$ it was found $|\widetilde{\varepsilon}-\varepsilon| / \varepsilon<5 \cdot 10^{-3}$.

\subsection{The crossover theory in ternary mixtures}

The crossover theory has been developed for binary mixtures. It is not self-evident that this theory is also valid for ternary mixtures. Moreover, the renormalization group $\varepsilon$ expansion predicts a dependence of the critical exponent of viscosity $Z_{\eta}$ upon the dimension of the considered system, [71]:

$$
Z_{\eta}=\frac{1}{19} \varepsilon+0.018 \varepsilon^{2}
$$

with $\varepsilon$ (note, in this case $\varepsilon$ is not the reduced temperature), the critical dimension $d_{c}=4$, and $\varepsilon=d_{c}-d$, where $d$ is the dimension of the system. Consequently, Eq.(4.37) predicts the value $\varepsilon / 19$ for a $3 d$-Ising system, in first order for $Z_{\eta}$. Taking into account the second order corrections, $Z_{\eta}=0.065$ results. This value has been predicted by the mode-coupling theory. In the case of tricritical point the critical dimension is reduced to $d_{c}=3$, as has been given by Pfeuty [72]. As a consequence, $\varepsilon=0$ and therefore $Z_{\eta}=0$. It seems that there is no critical divergence of the viscosity. However, the ternary system, that has been investigated it this work is of type $2 \mathrm{a}$ (see Section (2.4.2)) and belongs to the same universality class for dynamical properties as the binary fluids. Thus, there is no reason to treat the experimental data different from the binary mixtures. Furthermore, it can be expected, that the crossover corrections are likewise valid. 



\section{Experimental Verification of Dynamic Scaling Theories and Discussions}

In the following chapter, the results of measurements of dynamic light scattering, shear viscosity and ultrasonic spectroscopy as well as the data evaluation procedures are presented. The first Section describes the strategy in the fitting procedure of the scaling function within the framework of crossover theories as well as the Bhattacharjee-Ferrell theory. The second section lists the investigated substances and critical systems as well as their preparation. The third, fourth and fifth sections present the final results of the studies, classified by the complexity of the ultrasonic attenuation spectra: systems without or with one additional noncritical relaxation term, systems with complex background contributions, and ternary mixtures. The sixth section deals with correlations, e.g. with relations between quantities of different critical systems.

\subsection{Strategies of verifying the scaling function}

The formalism for the verification of the critical parameters and the scaling function presented in Chapter (4) calls for a specific treatment of experimental data within the framework of crossover formalisms as well as the Bhattacharjee-Ferrell theory of critical sound attenuation, Fig.(5.1). In principle, the scaling function can be derived directly from the ultrasonic measurements, by taking into account the Bhattacharjee-Ferrell theory. However, due to effects of critical slowing near $T_{c}$ as well as the enormously increasing attenuation coefficient toward low frequencies, only the high frequency part of the critical contribution to the ultrasonic spectra is obtainable from attenuation coefficient measurements. Therefore $\Gamma_{U S}$ is only inaccurately known from acoustical spectrometry. For this reason and in order to reduce the number of unknown parameters in ultrasonic spectroscopy, the relaxation rate of order-parameter fluctuations has been additionally determined by shear viscosity and mutual diffusion coefficient measurements. Consequently, no distinction between the relaxation rate of critical fluctuations from ultrasonic measurements, $\Gamma_{U S}$, and that from dynamic light scattering, $\Gamma_{D L S}$, will be made in the following:

$$
\Gamma_{0}^{D L S} \stackrel{!}{=} \Gamma_{0}^{U S}
$$




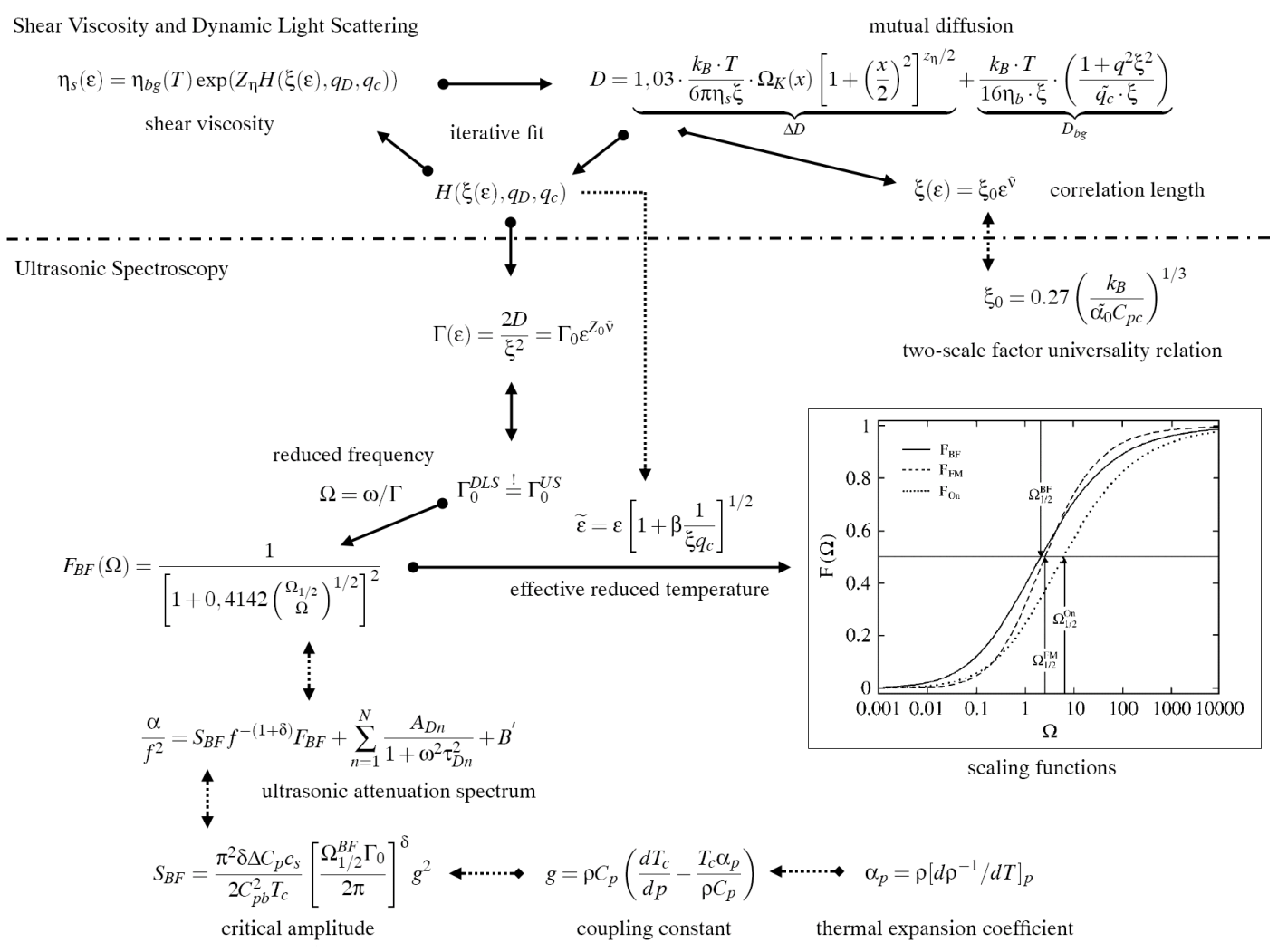

Figure 5.1: Scheme of fitting procedures for the derivation of the scaling function.

This step is justified, because of the assumptions made by Bhattacharjee and Ferrell. According to their theory, local concentration fluctuations detected in the light scattering experiments are controlled by diffusion, Eq.(4.10), of almost spherically shaped areas with concentration different from the mean. These fluctuating areas couple to the ultrasonic wave [19]. Hence, the assumption for the characteristic relaxation rate $\Gamma_{0}^{D L S}=\Gamma_{0}^{U S}=\Gamma_{0}$ can be done. In order to determine the characteristic relaxation rate $\Gamma_{0}$, dynamic light scattering and shear viscosity measurements have to be combined. Using the relation Eq.(4.11) and the Kawasaki-Ferrell relation, Eq.(4.10) can be rewritten to yield the relaxation rate of concentration fluctuations as a function of the diffusion coefficient and the shear viscosity:

$$
\Gamma=\frac{k_{B} T}{72 \pi^{2} \eta_{s}^{2} D^{3}}
$$

The mutual diffusion coefficient as well as the fluctuation correlation length $\xi$ can be derived from dynamic light scattering, according to the Eqs.(4.35). Both quantities are 
related by the dynamic scaling hypothesis [33], [34], [22], [78] presented in Eq.(4.11). However, crossover corrections have also to be taken into account. The crossover function $H\left(\xi(\varepsilon), q_{c}, q_{D}\right)$ contains an explicit dependence upon the fluctuation correlation length $\xi$ and on the cut-off wave numbers $q_{c}$ and $q_{D}$. Parameters $\xi, q_{D}$ and $q_{c}$ also control the mutual diffusion coefficient according to Eq.(4.35). Therefore, with the aid of an iterative fitting procedure the complicated expression of the crossover function $H$ in Eq.(4.31) has to be applied simultaneously to the experimental shear viscosity and dynamic light scattering data. Only this kind of data treatment allows to fulfil Eqs.(4.31) and (4.35) simultaneously and to thus yield consistent results for the parameters $\xi_{0}, q_{c}$ and $q_{D}$. Finally, the value for $\Gamma_{0}$ determined in this way can be used in Eq.(4.16), that represents the critical attenuation contribution and controls the frequency as well as temperature dependence of the scaling function $F(\Omega)$. However, the critical amplitude is still an adjustable parameter. It weakly depends upon frequency due to the small critical exponent $\delta=\tilde{\alpha_{0}} /\left(Z_{0} \tilde{\mathrm{v}}\right)$ in Eq.(4.17). If experimental heat capacity data are available, it is possible to calculate, with the aid of the Eq.(4.17) the amplitude $S_{B F}$ in Eq.(4.16) from thermodynamical quantities of critical mixture. Alternatively it is possible to extract the the amplitude from the ultrasonic spectra and to estimate the adiabatic coupling constant $g$ according to Eq.(4.18). Furthermore, the amplitude of the correlation length $\xi$ can be additionally verified with the help of heat capacity data using the so-called two-scale-factor universality relation [79]:

$$
\xi_{0}=\left(\frac{k_{B} X}{A^{+}}\right)^{1 / 3}
$$

with Boltzmann's constant $k_{B}$, and with $X=(1.966 \pm 0.017) \cdot 10^{-2}$ and $X=(1.88 \pm$ $0.015) \cdot 10^{-2}$ from renormalization-group and series calculations, respectively, [79], [80], with the amplitude factor $A^{+}$of the singular contribution to the heat capacity at constant pressure in the one-phase regime [74], [75],

$$
C_{p}=\frac{A^{+}}{\tilde{\alpha}_{0}} \varepsilon^{-\tilde{\alpha}_{0}}\left(1+D^{+} \varepsilon^{\Delta}\right)+E^{+} \varepsilon+B^{+}
$$

with the heat critical exponent $\tilde{\alpha_{0}}=0.11$ and $\Delta=0.51 \pm 0.03$. However, as was mentioned in Section (4.4) the values for $\xi_{0}$ and $q_{c}$ lead to noticeable changes of the reduced temperatures $\varepsilon$ in the treatment of ultrasonic measurements. Hence, in the determination of the scaling function the reduced temperatures $\varepsilon$ have to be corrected to get the effective reduced temperature $\tilde{\varepsilon}$ according to Eq.(4.36). The fitting procedures applied on the basis of the dynamic scaling hypothesis and the crossover formalism are summarized schematically in Fig.(5.1). 


\subsection{Preparation of critical mixtures}

\begin{tabular}{|c|c|c|c|c|}
\hline substance & formula & supplier & purity & $\begin{array}{l}\text { molar mass } \\
{[\mathrm{g} / \mathrm{mol}]}\end{array}$ \\
\hline$n$-butanol ${ }^{r}$ & $\mathrm{C}_{4} \mathrm{H}_{10} \mathrm{O}$ & Fluka & $\geq 99 \%$ & 74.12 \\
\hline water ${ }^{r}$ & $\mathrm{H}_{2} \mathrm{O}$ & $\begin{array}{l}\text { deionized } \\
\text { and bidistilled }\end{array}$ & & 18.00 \\
\hline nitromethane & $\mathrm{CH}_{3} \mathrm{NO}_{2}$ & Fluka & $\geq 99 \%$ & 61.04 \\
\hline$n$-pentanol & $\mathrm{C}_{5} \mathrm{H}_{12} \mathrm{O}$ & Fluka & $\geq 99 \%$ & 88.15 \\
\hline nitroethane & $\mathrm{C}_{2} \mathrm{H}_{5} \mathrm{NO}_{2}$ & Aldrich & $\geq 99.5 \%$ & 75.08 \\
\hline 3-methylpentane & $\mathrm{C}_{6} \mathrm{H}_{14}$ & Aldrich & $\geq 99 \%$ & 86.20 \\
\hline cyclohexane & $\mathrm{C}_{6} \mathrm{H}_{12}$ & Aldrich & $\geq 99.5 \%$ & 84.16 \\
\hline methanol & $\mathrm{CH}_{4} \mathrm{O}$ & Fluka & $\geq 99.8 \%$ & 32.04 \\
\hline$n$-hexane & $\mathrm{C}_{6} \mathrm{H}_{14}$ & Aldrich & $\geq 99 \%$ & 86.18 \\
\hline ethanol & $\mathrm{C}_{2} \mathrm{H}_{6} \mathrm{O}$ & Fluka & $\geq 99 \%$ & 46.07 \\
\hline n-dodecane & $\mathrm{C}_{12} \mathrm{H}_{26}$ & Aldrich & $\geq 99 \%$ & 170.34 \\
\hline isobutoxyethanol & $\mathrm{C}_{6} \mathrm{H}_{14} \mathrm{O}_{2}$ & Wako & $\geq 97 \%$ & 118.17 \\
\hline 2,6-dimethylpyridine & $\mathrm{C}_{7} \mathrm{H}_{9} \mathrm{~N}$ & Aldrich & $\geq 99 \%$ & 107.15 \\
\hline triethylamine & $\mathrm{C}_{6} \mathrm{H}_{15} \mathrm{~N}$ & Aldrich & $\geq 99 \%$ & 101.19 \\
\hline
\end{tabular}

Table 5.1: Substances used: index ${ }^{r}$ denotes substance also used for reference measurements and calibrations.

All non-aqueous constituents of the mixtures presented in Table (5.1), have been used as delivered, except for the substance isobutoxyethanol which was additionally purified by fractional distillation at $318 \mathrm{~K}$ and at a reduced pressure of $23 \mathrm{mbar}$ in a concentric tube column of 64 real plates. This distillation procedure has been performed several times, to get isobutoxyethanol surfactant as pure as possible. Before preparing mixtures, the constituents were degassed in an ultrasonic bath or vacuum oven. This preparation step was done to avoid gas bubbles that might grow in the cells during measurements. In order to avoid preferential evaporation, the degassing in a vacuum oven has been performed before preparing the mixtures. Finally, mixtures have been prepared under nitrogen gas atmosphere by weighing appropriate amounts of the constituents into suitable flasks. Uptake of water from the air was avoided thereby. The critical point of a mixture was determined visually by taking into account the criterion of critical opalescence as well as the criterion of equal volume (Section 2.3.5). Additionally, the existence of the critical point has been determined by densitometer measurements (Anton Paar 5000) in Section (3.4). In the case of ultrasonic measurements, calibrations for resonator losses were performed afterwards, using reference liquids: butanol for mixtures listed in Table (5.2) and deionized bidistilled water for aqueous mixtures listed in Table (5.3). In addition, water has been 
used to determine the cell length of the resonators. The critical parameters like critical mole fraction $x_{c}$ and the critical temperature $T_{c}$ of investigated systems are given in Table (5.2) for binary systems without or with one additional noncritical relaxation term, and in Table (5.3) for binary systems with complex background contributions.

\begin{tabular}{lcc}
\hline \hline critical mixture & $\begin{array}{c}\text { critical conc. } x_{c} \text { in mole frc. critical Temp. } T_{c} \\
\text { of first component }\end{array}$ & {$[\mathrm{K}]$} \\
\hline$n$-pentanol-nitromethane (PE-NM) & 0.385 & 300.95 \\
nitroethane-3-methylpentane (NE-3MP) & 0.500 & 299.68 \\
nitroethane-cyclohexane (NE-CH) & 0.452 & 296.46 \\
methanol-hexane (ME-HEX) & 0.500 & 307.74 \\
ethanol-dodecane (ET-DOD) & 0.687 & 285.82 \\
\hline
\end{tabular}

Table 5.2: Critical composition and temperature of binary systems without or with one additional noncritical relaxation term.

\begin{tabular}{lcc}
\hline \hline critical mixture & $\begin{array}{c}\text { critical conc. } x_{c} \text { in mole frc. } \\
\text { of fritical Temp. } T_{c}\end{array}$ \\
\hline isobutoxyethanol-water $\left(i-\mathrm{C}_{4} \mathrm{E}_{1} / \mathrm{H}_{2} \mathrm{O}\right)$ & 0.070 & {$[\mathrm{~K}]$} \\
2,6-dimethylpyridine-water $\left(2,6-\mathrm{DMP}-\mathrm{H}_{2} \mathrm{O}\right)$ & 0.065 & 298.10 \\
triethylamine-water $\left(\mathrm{TEA}-\mathrm{H}_{2} \mathrm{O}\right)$ & 0.076 & 306.83 \\
\hline \hline
\end{tabular}

Table 5.3: Critical composition and temperature of binary systems with complex background contributions.

\subsection{Binary systems without and with one additional noncritical relaxation term}

Because of the complexity of the Bhattacharjee-Ferrell formalism, it is useful to first study critical systems for which additional chemical relaxation contributions in the range of frequencies of measurements are not expected in the ultrasonic spectra. It was thus interesting to perform investigations into the critical dynamics of binary mixtures of critical composition, such as $n$-pentanol-nitromethane (n-PE-NM) [76], nitroethane-cyclohexane (NE-CH) [90], methanol-cyclohexane (ME-HEX) [93], ethanol-dodecane (ET-DOD) [99], as well as nitroethane-3-methylpentane (NE-3MP) [86], for which no such contributions from elementary molecular processes are expected. 


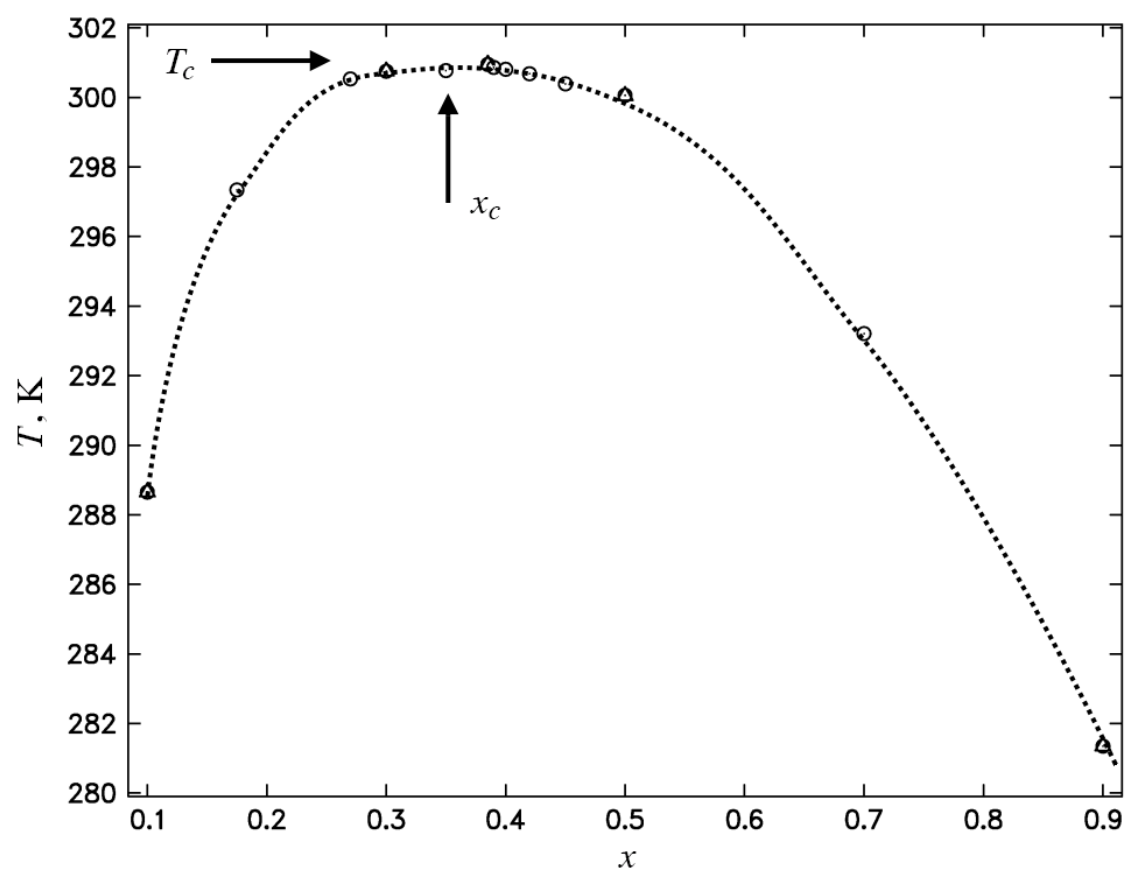

Figure 5.2: Phase transition curve of $n$-pentanol-nitromethane, mole fraction $x$ versus temperature, with the critical concentration of mole fraction $x_{c}=0.385$ and the critical temperature $T_{c}=300.45: \triangle$ represent literature data, [81].

\subsubsection{Phase diagram and critical temperature.}

An example of a binodale curve of the binary system ( $n$-PE-NM) [76] at mole fractions $x$ of alcohol between 0.3 and 0.45 is shown in Fig.(5.2). The critical point can be derived from density measurements shown, as an example, for the (TEA- $\left.\mathrm{H}_{2} \mathrm{O}\right)$ mixture of critical composition in Fig.(5.3), as well as from the half-power bandwidth $\Delta f_{r}$ of a resonance of an ultrasonic resonator filled with the sample. An example of $\Delta f_{r}$ is displayed in Fig.(5.4) for the binary critical mixture (ME-HEX). The nonlinear increase in the half-power bandwidth, when approaching the consolute point, is an indicator of the influence of critical fluctuations. At the critical point, the half-power bandwidth data reach a maximum. In the multiphase regime the values of the half-power bandwidth of a resonance decrease. 


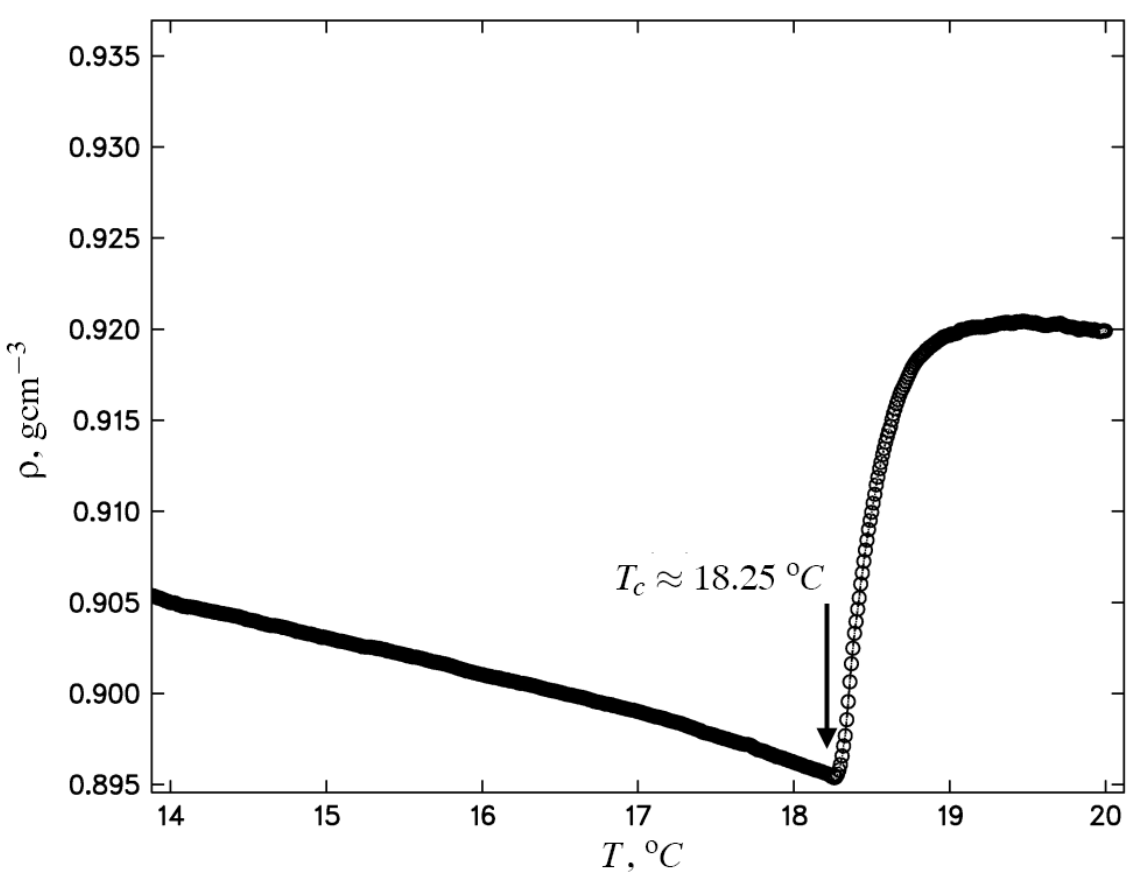

Figure 5.3: Density of the binary system triethylamine-water of critical composition with lower critical temperature.

\subsubsection{Dynamic light scattering, shear viscosity $\eta_{s}$ and characteristic relaxation rate $\Gamma_{0}$}

As mentioned in the previous section, in order to reduce the number of unknown parameters, ultrasonic spectrometry has been complemented by the dynamic light scattering and shear viscosity measurements. The lifetime of critical fluctuations $\tau_{\xi}$ has been determined by dynamic light scattering and the shear viscosity measurements as presented in Chapter (2). The shear viscosity has been measured as a function of temperature using an capillary and a falling ball viscosimeter. These two methods have been used to complement each other as well as to control the shear rate dependence in $\eta_{s}$. In the (NE-CH) system it was found that the shear rate dependence in the viscosity data is within the limits of experimental errors $\Delta \eta_{s} / \eta_{s}=0.02$. Both methods of measurements agree with one other as is shown by the data in Fig.(5.5). However, in order to carefully treat the capillary viscosimeter data, the shear rate corrections have to be taken into account. Those corrections have to be performed, because the critical temperature close to the consolute point changes due to macroscopic non-linear effects. The relation between geometrical parameters of the capillary and the shear rate was given by Kohlrausch: 


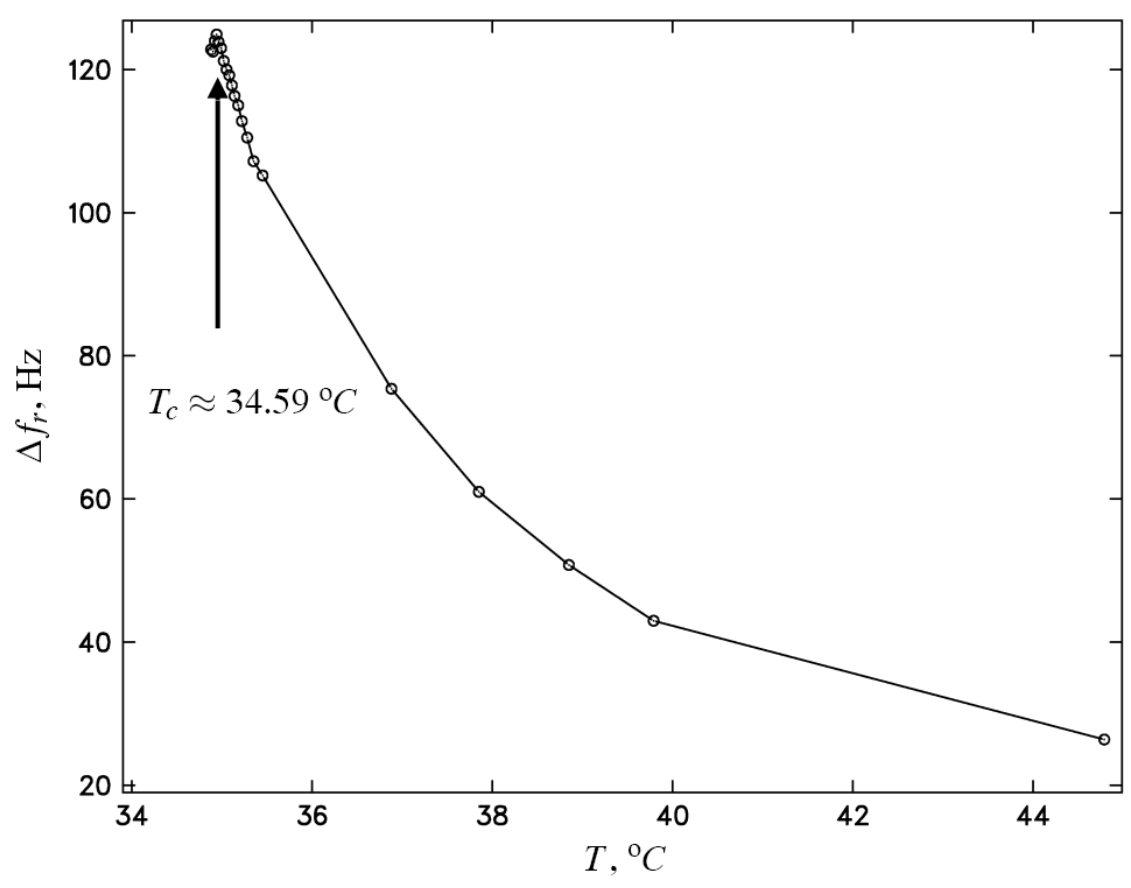

Figure 5.4: Half-power bandwidth $\Delta f_{r}$ in $\mathrm{Hz}$ of an acoustical resonance at $790 \mathrm{kHz}$ of a cylindrical cavity resonator filled with the mixture methanol- $n$-hexane versus temperature $T$ in ${ }^{\circ} \mathrm{C}$. The system exhibits an upper critical temperature.

$$
\dot{\gamma}=\frac{4 \Delta V}{\pi \cdot \Delta t \cdot R^{3}}
$$

where $R$ denotes the radius of the capillary, $\Delta V$ the volume of the fluid running during $\Delta t$, the duration of measurement. The kinematic viscosity is defined by:

$$
\eta_{\text {kin }}=\underbrace{\frac{\pi \cdot R^{4} \cdot g}{8 \cdot l \cdot \Delta V} \cdot h}_{\mathcal{K} \text {-apparative constant }} \cdot \Delta t,
$$

with the length of capillary $l$, the falling height $h$ and $g$ the gravitation constant. Finally, the so-called effective shear rate results:

$$
\dot{\gamma}=\frac{\partial u}{\partial x} \approx \mathcal{K}^{-1} \cdot \frac{R \cdot g \cdot h}{2} \cdot \Delta t^{-1}
$$




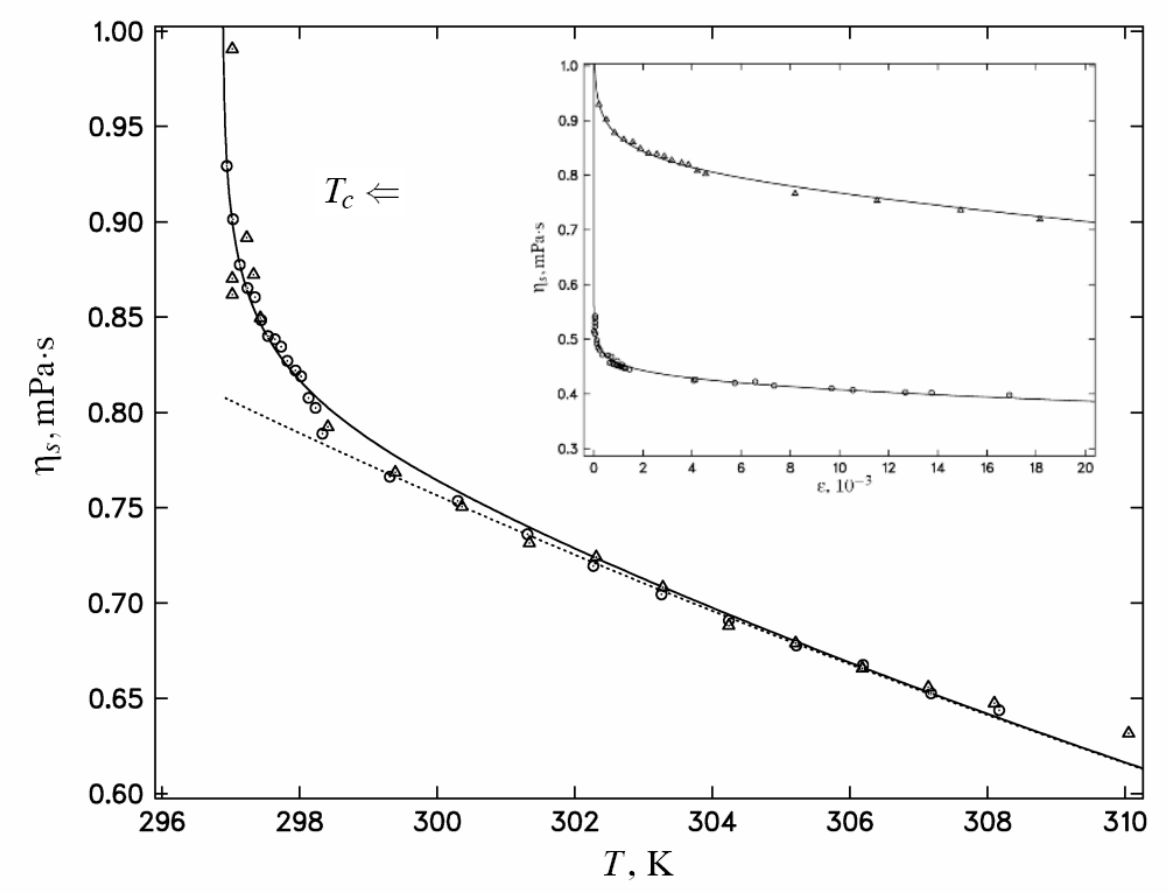

Figure 5.5: Shear viscosity $\eta_{s}$ of the (NE-CH) mixture of critical composition versus absolute temperature: The full curve and the dashed line are graphs of the shear viscosity and the background contribution of the viscosity, respectively. In the inset, the shear viscosity data of the (NE-3MP) system $(\bullet)$ are given along with those of the (NE-CH) mixture of critical composition $(\mathbf{\Lambda})$.

This effective shear rate has an influence on the critical temperature $T_{c}$. With $S$ as a correction parameter, the critical temperature has to be written as:

$$
T_{c}(S)=\frac{T_{c}(S=0)}{1 \pm 0.0832 \cdot\left(\frac{16 \eta \xi^{3} \cdot S}{k_{b} T}\right)}
$$

where in the equation $(+)$ refers to an upper critical point (systems in Table (5.2)) and $(-)$ to a lower critical point (systems in Table (5.3)). Independent on the magnitude of shear rate dependence, the 
corrections were taken into account automatically in every fitting procedure. The shear viscosity data of the system (NE-3MP) [86] are presented in the inset of Fig.(5.5). The viscosity data have been completed by literature data [87]. The experimental data display a background contribution, slowly decreasing with the temperature close to the critical point, and a singular part that distinctly increases when approaching $T_{c}$. The background contribution $\eta_{b g}$ has been fitted by the Eq.(4.29) and the complete viscosity data by the Eq.(4.31), tak-

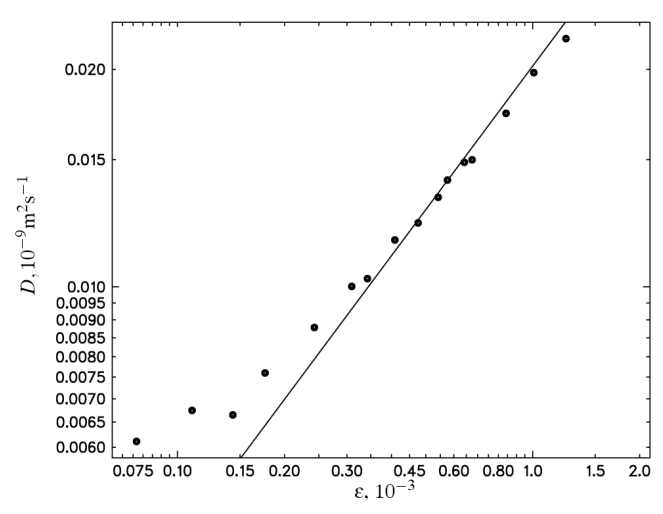

Figure 5.6: Bilogarithmic plot $D-\varepsilon$ of (n-PE-NM), in which the line represents power law behavior, Eq.(5.10). ing into account the crossover function $H\left(\xi(\varepsilon), q_{D}, q_{c}\right)$. By simultaneously fitting the correlation time $\tau_{c}$ and the shear viscosity data, the fluctuation correlation length $\xi$ was determined. In Fig.(5.7) the bilogarithmic

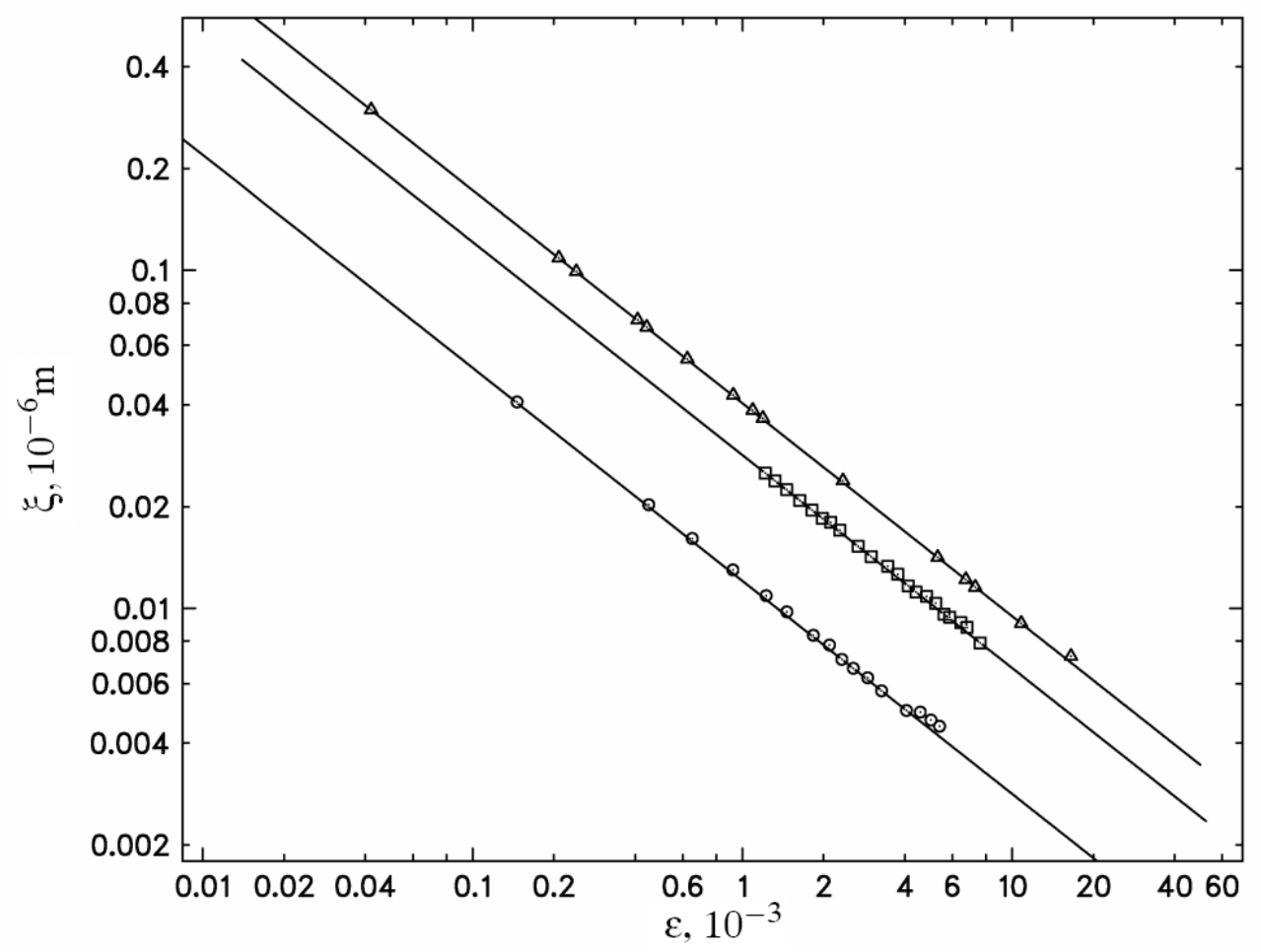

Figure 5.7: Fluctuation correlation length of the systems (NE-CH) $\circ$, (NE-3MP) $\triangle$, (ETDOD) $\square$ versus reduced temperature: lines represent the power law $\xi=\xi_{0} \varepsilon^{\tilde{v}}$. 


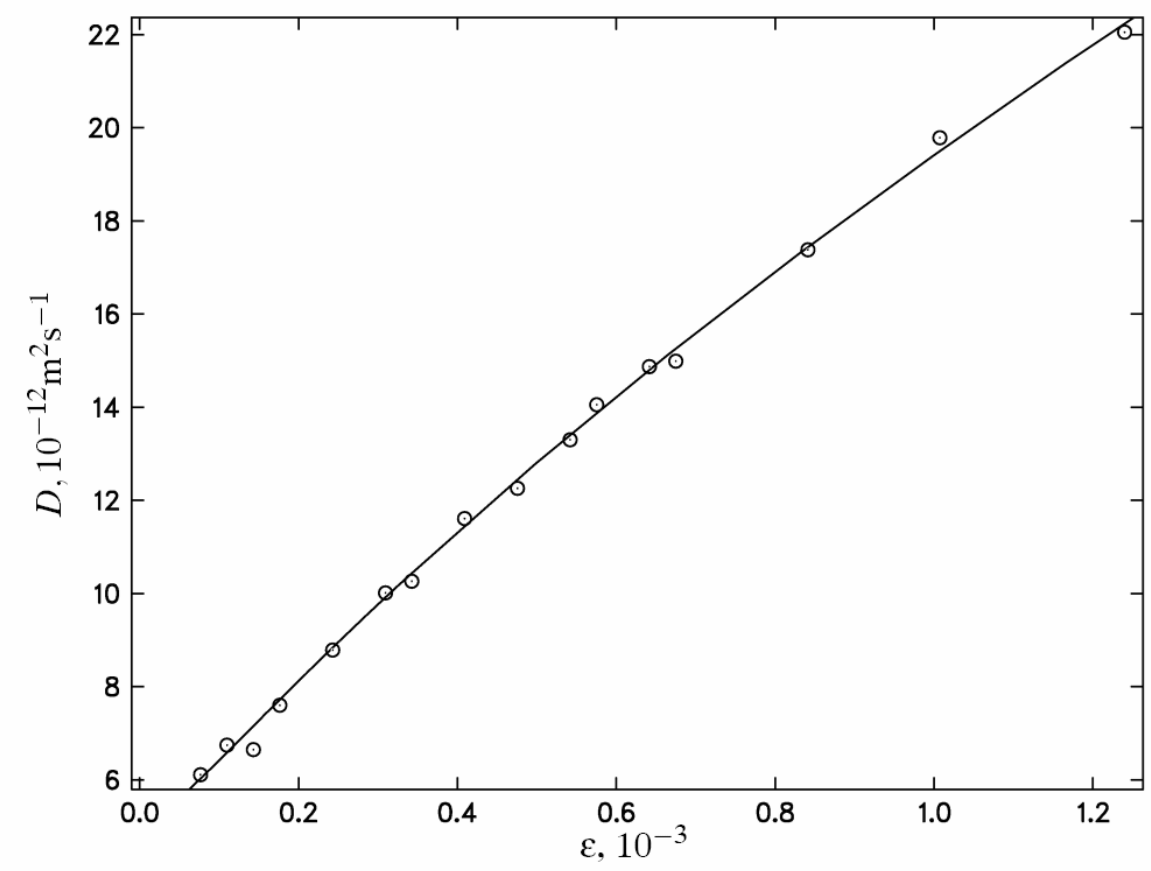

Figure 5.8: Plot of the diffusion coefficient $D$ of the binary system ( $n$-PE-NM) versus reduced temperature $\varepsilon$. The line is the graph of Eq.(4.35).

plots of the correlation length $\xi$ versus $\varepsilon$ of the systems (NE-CH), (NE-3MP), and (ETDOD) are compared with each other. According to, the frequently used power law:

$$
\xi=\xi_{0} \varepsilon^{\tilde{v}}
$$

with the critical exponent $\tilde{v}$, and the amplitudes $\xi_{0}=0.160 \mathrm{~nm}$ for $(\mathrm{NE}-\mathrm{CH}), \xi_{0}=0.230$ $\mathrm{nm}$ for (NE-3MP) and $\xi_{0}=0.370 \mathrm{~nm}$ for (ET-DOD). A plot of the diffusion coefficient versus reduced temperature of the example (n-PE-NM) is given in Fig.(5.8). The mutual diffusion coefficient, is also assumed to consist of a singular part $\Delta D$ and a background part $D_{b g}$, as was presented in Eq.(4.33), and likewise depends on parameters $\xi, q_{D}$, and $q_{c}$. Another bilogarithmic plot of the diffusion coefficient of the same mixture versus reduced temperature $\varepsilon$ is given in Fig.(5.6), where also the power law:

$$
D=D_{0} \varepsilon^{v^{*}}
$$

is shown . Here $v^{*}=0.664$ denotes the critical exponent from mode coupling theory. 
From a least-squares fitting procedure $D_{0}=(11.1 \pm 0.5) \cdot 10^{-10} \mathrm{~m}^{2} \mathrm{~s}^{-1}$ followed. However, the diffusion coefficient data exhibit some deviations from the power law behavior. These deviations may result from the insufficient temperature control $( \pm 0.03)$ in the measurements. Finally, according to Eq.(5.2) taking into account crossover effects, the characteristic relaxation rate $\Gamma_{0}(\varepsilon)$ of order parameter fluctuations can be determined according to the dynamic scaling hypothesis, Eq.(4.11). Over a significant range of reduced temperatures the relaxation rate follows power law Eq.(4.11) with the theoretical critical exponent $Z_{0} \tilde{\mathrm{V}}$. An example of bilogarithmic plot of $\Gamma_{0}(\varepsilon)$ of the system $(\mathrm{NE}-\mathrm{CH})$ is given in Fig.(5.9) The resulting parameters for other systems investigated by dynamic

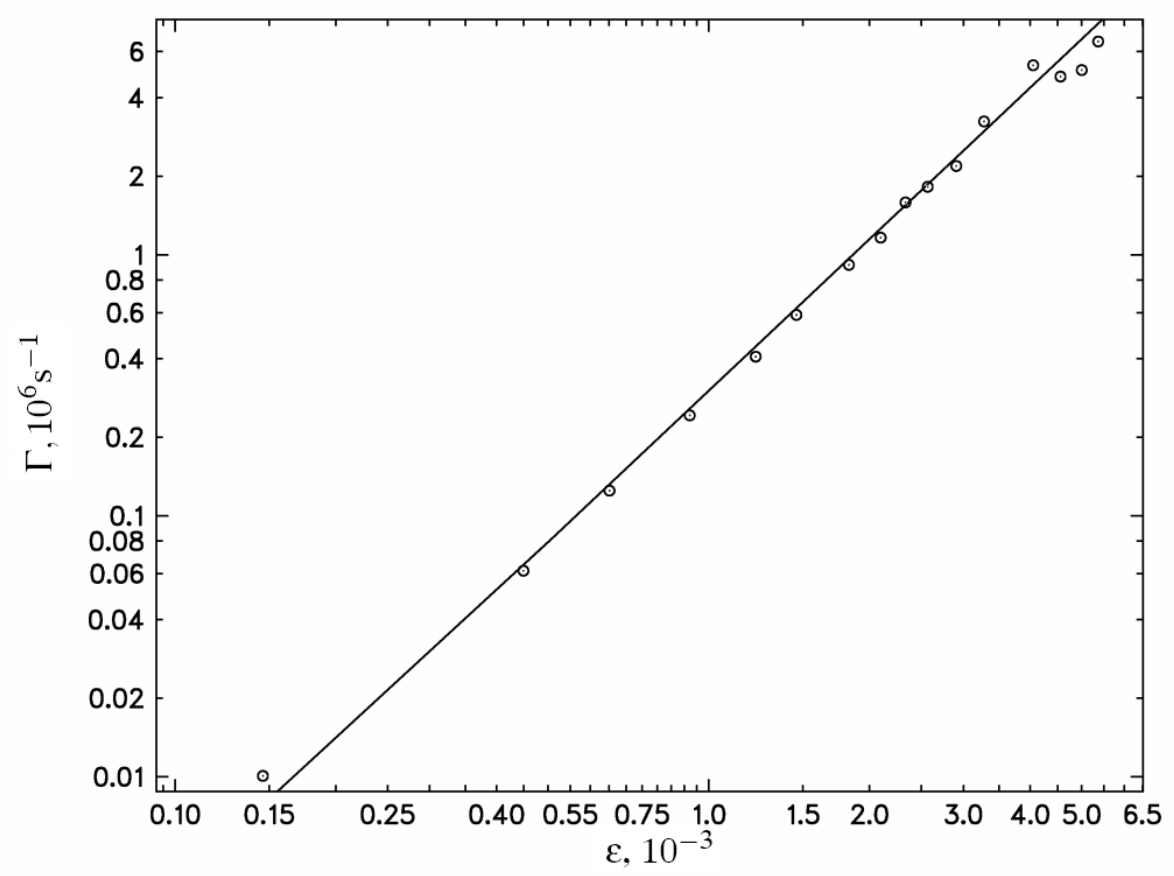

Figure 5.9: Bilogarithmic plot of the relaxation rate $\Gamma$ of critical fluctuations versus reduced temperature $\varepsilon$ of the binary system (NE-CH): The line represents $\Gamma=\Gamma_{0} \varepsilon^{Z_{0} \tilde{v}}$, with $\Gamma_{0}=156 \cdot 10^{9} \mathrm{~s}^{-1}$.

light scattering and shear viscosity measurements are listed in Table (5.9).

\subsection{Fluctuation correlation length}

As presented in Section (5.1), the amplitude $\xi_{0}$ of the fluctuation correlation length, obtained from the shear viscosity and dynamic light scattering measurements, can be ad- 


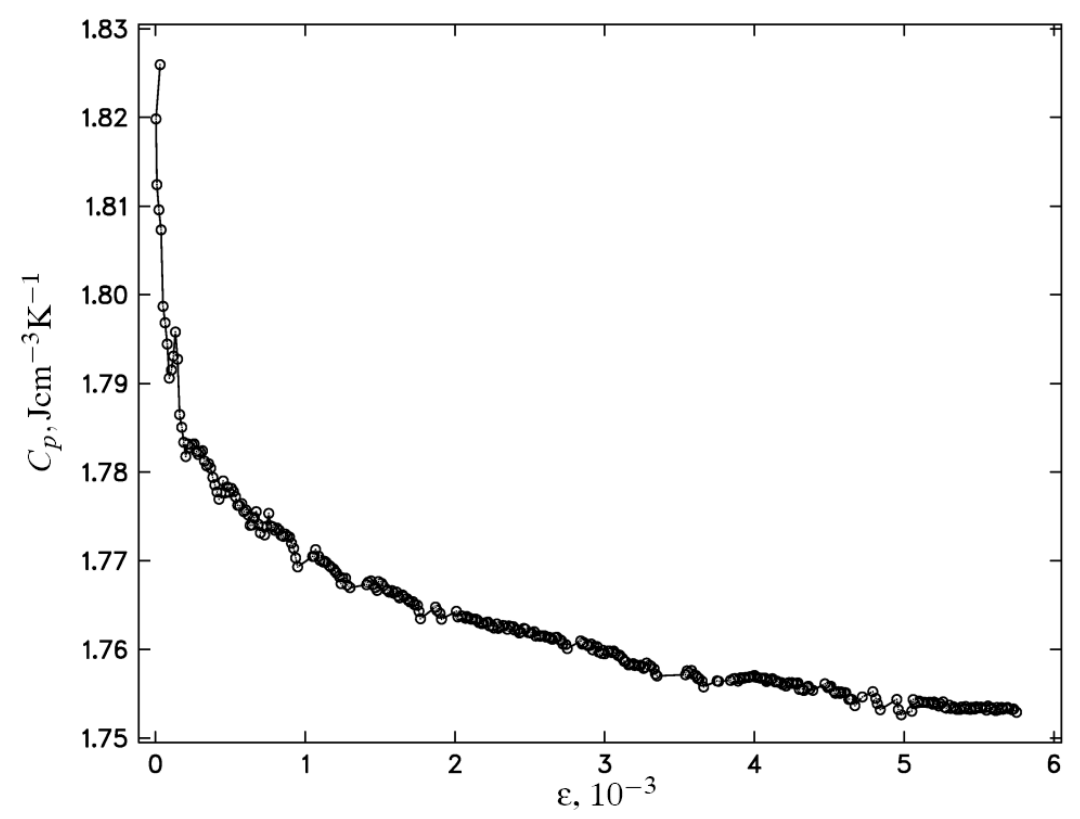

Figure 5.10: DSC heat capacity data as obtained from one run in the downscan mode for the methanol- $n$-hexane mixture of critical composition near $T_{c}$ plotted versus reduced temperature $\varepsilon$ : (scan-rate $1.05 \mathrm{Kh}^{-1}$ ).

ditionally verified or disproved with the aid of the two-scale factor universality relation. This evaluation procedure can be done if heat capacity data are available. An example of heat capacity data from one run of a differential scanning calorimeter for the binary mixture (ME-HEX) is shown in Fig.(5.10). According to Eq.(5.4), a nonlinear least-squares regression analysis of the data in Fig.(5.10), including $C_{p}$ data in an extended temperature range $\left(T_{c} \leq T \leq T_{c}+10 \mathrm{~K}\right)$, yields $A^{+} / \alpha_{0}=(6.99 \pm 0.02) \times 10^{-2} \mathrm{~J}$ $/\left(\mathrm{cm}^{3} \mathrm{~K}\right), D^{+}=5.6 \pm 0.1, E^{+}=-(3.1 \pm 0.1) \mathrm{J} /\left(\mathrm{cm}^{3} \mathrm{~K}\right)$, and $B^{+}=(1.600 \pm 0.002) \mathrm{J}$ $/\left(\mathrm{cm}^{3} \mathrm{~K}\right)$. Reevaluation of heat capacities for the (ME-HEX) system measured with an adiabatic calorimeter [92] yielded the somewhat higher value $A^{+} / \alpha_{0}=(8 \pm 1) \times 10^{-2}$ $\mathrm{J} /\left(\mathrm{cm}^{3} \mathrm{~K}\right)$, in fair agreement with the result from the present measurements. Using $A^{+} / \alpha_{0}=6.99 \times 10^{-2} \mathrm{~J} /\left(\mathrm{cm}^{3} \mathrm{~K}\right)$ the amplitude $\xi_{0}=0.32 \mathrm{~nm}$ follows from Eq.(5.3) which perfectly agrees with $\xi_{0}=(0.33 \pm 0.03) \mathrm{nm}$ as resulting from the combined evaluation of the shear viscosity and dynamic light scattering data. This agreement may be taken as an indication of consistency of the evaluation procedure. If heat capacity data were available from measurements or from literature, such calculations have been performed for every mixture (see results summarized in Table (5.9)). 


\subsection{Ultrasonic spectrometry}

At frequencies $f$ between $180 \mathrm{kHz}$ and $500 \mathrm{MHz}$ broad-band ultrasonic spectra of binary mixtures have been measured to verify non-existence of additional relaxation terms in the measuring range as well as to determine the background contribution $\left(\alpha / f^{2}\right)_{b g}$. At three temperatures the ultrasonic absorption spectrum of the (NE-CH) mixture of critical composition is shown in the homogenous phase in Fig.(5.11). In order to accentuate the low frequency part of the spectra, data are shown in the frequency normalized format

$$
\left(\alpha / f^{2}\right)=(\alpha \lambda) /\left(f c_{s}\right)
$$

In this format the data approach asymptotically the frequency independent value (data extrapolated to frequencies far above the frequency range of measurements):

$$
\lim _{f \rightarrow \infty}\left(\alpha / f^{2}\right)=\left(\alpha / f^{2}\right)_{b g}=B^{\prime}(T),
$$

The constant frequency-independent contribution which is indicated in Fig.(5.11) has to be taken into account in the description of the ultrasonic spectra. Assuming, that the critical fluctuations contribute additively to the ultrasonic spectrum, the spectral description of measured data can be displayed as a sum of the critical contribution $R_{c}(f)=(\alpha \lambda)_{c}$ Eq.(4.16), governed by the dynamic scaling theory, and the background contribution $(\alpha \lambda)_{b g}$ :

$$
(\alpha \lambda)=R_{c}(f)+B f
$$

In Fig.(5.12) the broad band spectra of the critical mixture (n-PE-NM) at three temperatures are shown. The strong increase of $\left(\alpha / f^{2}\right)$ data with decreasing frequency and approaching of the critical temperature, indicates the critical contribution to the spectra. Obviously the critical fluctuations contribute extensively to the sound attenuation spectra in the low frequency range. Therefore, for the purpose of verifying the dynamic scaling model, the low frequency behavior between $180 \mathrm{kHz}$ and $1.8 \mathrm{MHz}$ has been studied with more details. For measurements at those frequencies a plano-concave resonator method has been employed which enables $\alpha$ to be determined as a function of frequency and temperature with a minimum risk of effects from mechanical stress of the cell as $T$ is varied. Examples of cavity resonator measurements are displayed in Fig.(5.13) for the systems (NE-3MP) and ( $n$-PE-NM). The low frequency spectra demonstrate a non-linear increase of the sound attenuation coefficient $\alpha$ with decreasing $f$, caused by critical contributions. Unfortunately, due to this behavior the number of measurable data points decreases as $T_{c}$ is approached, especially in the case of (n-PE-NM), for which the sound attenuation is 


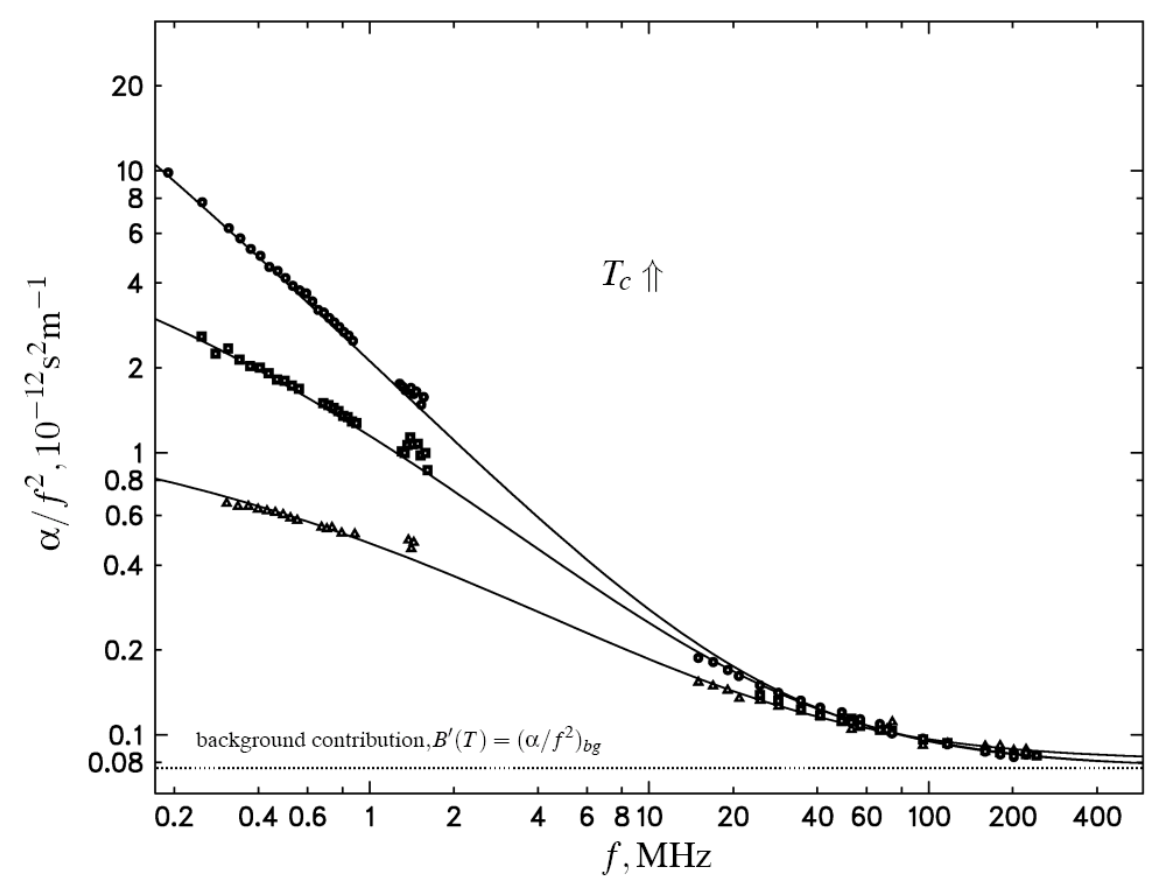

Figure 5.11: Frequency normalized ultrasonic attenuation spectra for the (NE-CH) mixture of critical composition at three temperatures: $(\Delta): 28.3{ }^{\circ} \mathrm{C},(\boldsymbol{\square}): 25.6{ }^{\circ} \mathrm{C},(\bullet): 24.0$ ${ }^{\mathrm{o}} \mathrm{C}$.

exceptionally large. According to the Bhattacharjee-Ferrell theory the scaling function $F_{B F}(\Omega)$ can be derived, rewriting Eq.(4.25) as ratio:

$$
F_{B F}(\Omega)=\underbrace{\frac{c_{s}\left(T_{c}\right) A\left(T_{c}\right)}{c_{s}(T) A(T)}}_{F_{B F}^{*}} \cdot \frac{(\alpha \lambda)_{c}(f, T)}{(\alpha \lambda)_{c}\left(f, T_{c}\right)}=F_{B F}^{*} \cdot \frac{(\alpha \lambda)(f, T)-(\alpha \lambda)_{b g}(f, T)}{(\alpha \lambda)(f, T)-(\alpha \lambda)_{b g}\left(f, T_{c}\right)}
$$

Usually, it is assumed that the temperature dependence in the sound velocity $c_{s}$ and also in the amplitude $A$ of the attenuation coefficient is sufficiently small to be neglected so that $F_{B F}^{*}=1$ can be used in Eq.(5.14), as originally proposed by Bhattacharjee and Ferrell. This precondition, however, is not always fulfilled. A closer examination of the binary critical mixture (NE-CH) points at an unusual dependence of the critical amplitude $S_{B F}$ on temperature [90]. In the next section this amplitude behavior will be discussed with more details. Moreover, for (ME-HEX) as well as (ET-DOD) it has been found that, although both considered constituents of the binary systems do not reveal indications of a chemical relaxation in pure liquids, the mixture indicates a relaxation due to an alco- 


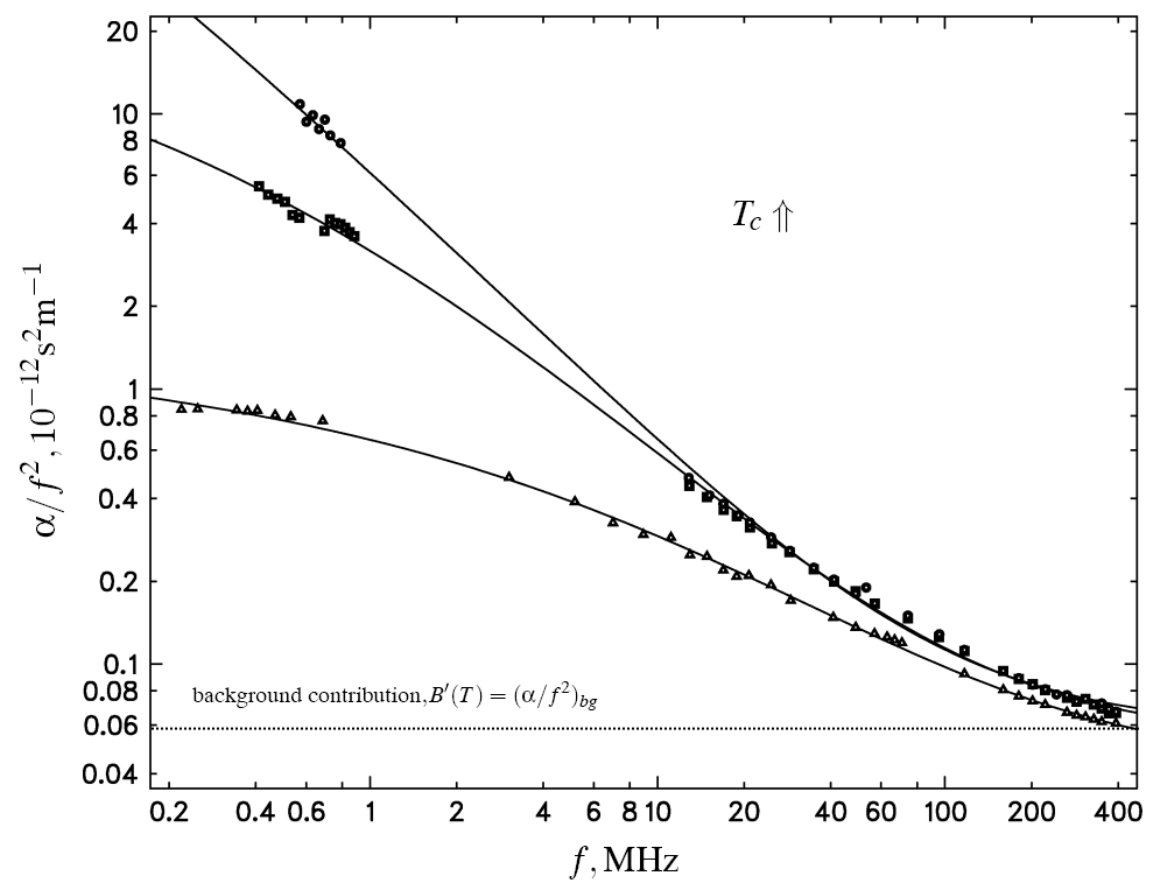

Figure 5.12: Frequency normalized ultrasonic attenuation spectra for the (PE-NM) mixture of critical composition at three temperatures: $(\triangle): 27.82{ }^{\circ} \mathrm{C},(\square): 29.42{ }^{\circ} \mathrm{C}$, $(\bullet): 34.84{ }^{\circ} \mathrm{C}$.

hol association mechanism. This indication is confirmed by literature data [91] as well as by present measurements of mixtures with noncritical composition (weight fraction of methanol 0.0074, with the (ME-HEX) system, and weight fraction of ethanol 0.008, with the system (ET-DOD)). A spectrum for the latter is shown in Fig.(5.14). A relaxation process, with relaxation time in the nanosecond range of has been found at $25{ }^{\circ} \mathrm{C}$ (2 ns (ME-HEX)), (19.1 ns (ET-DOD)). The inset in Fig.(5.14) represents a $(\alpha \lambda)$ plot. The existence of relaxation characteristics in the spectra means, that the background part $(\alpha \lambda)_{b g}$ can not be simply derived from high frequency attenuation coefficient extrapolation but has to be determined from $(\alpha \lambda)$ in a more sophisticated way. The additional relaxation term has to be taken into account in the background contribution, in Eq.(5.14). An alternative treatment of the additional chemical contribution to the sound attenuation spectra can be performed, if the temperature dependence of the relaxation time $\tau_{D}$ as well as of the Debye amplitude $A_{D}$ is known. In such cases the ultrasonic spectrum can be represented by following expression, according to Eq.(4.26), where the description of a ultrasonic spectrum has been extended by a Debye-term. 

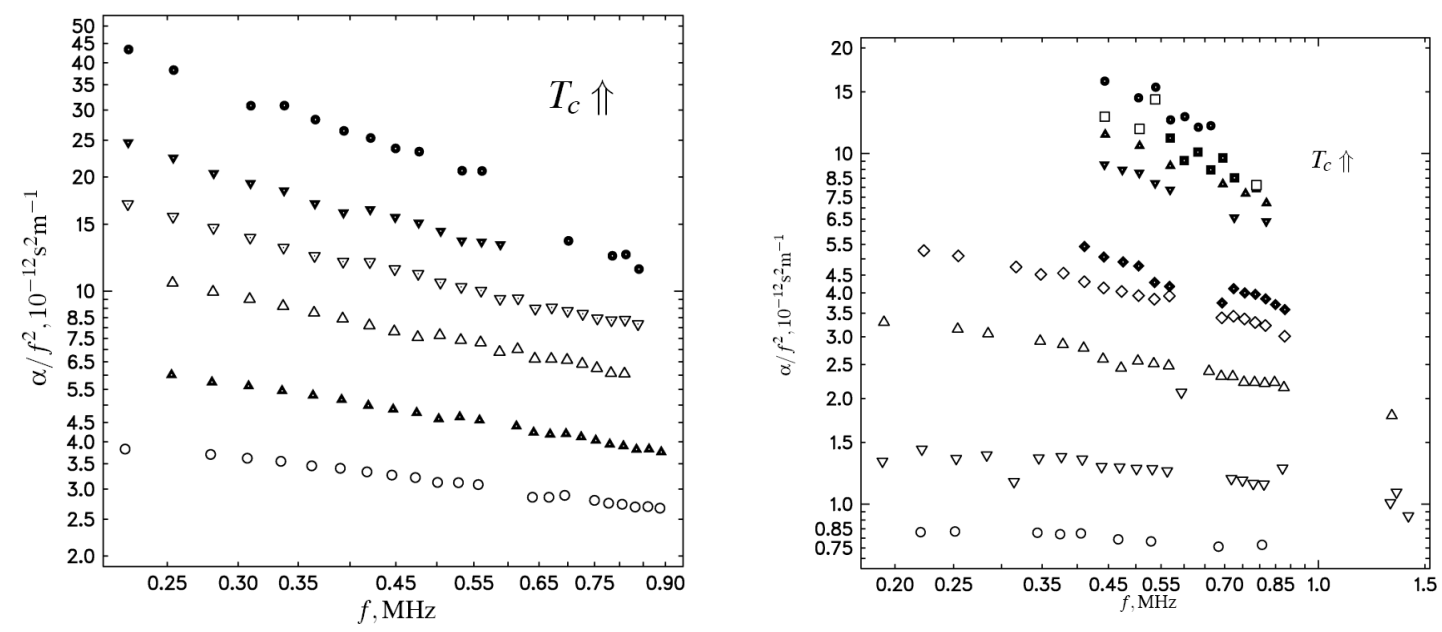

Figure 5.13: Ultrasonic attenuation spectra for mixtures of critical composition. Left Figure (NE-3MP): (o), $T=304.20 \mathrm{~K} ;(\boldsymbol{\Delta}), T=303.34 \mathrm{~K} ;(\triangle), T=302.20 \mathrm{~K} ;(\nabla), T=301.78 \mathrm{~K}$; $(\boldsymbol{\nabla}), T=301.34 \mathrm{~K} ;(\bullet), T \approx 299.68 \mathrm{~K}=T_{c}$; Right Figure $(n$-PE-NM): $(\circ), T=307.86 \mathrm{~K} ;(\nabla)$, $T=305.93 \mathrm{~K} ;(\triangle), T=303.98 \mathrm{~K} ;(\diamond), T=303.03 \mathrm{~K} ;(\diamond), T=302.57 \mathrm{~K} ;(\nabla), T=301.70 \mathrm{~K} ;(\boldsymbol{\Delta})$, $T=301.41 \mathrm{~K} ;(\boldsymbol{\square}), T=301.30 \mathrm{~K} ;(\square), T=301.22 \mathrm{~K} ;(\bullet), T \approx 300.97 \mathrm{~K}=T_{c}$.

Due to effects of critical slowing down near the consolute point $\left(x_{c}, T_{c}\right)$ as well as the dramatically increasing sound attenuation as shown in Fig.(5.13) in the case of the binary systems ( $n$-PE-NM) and (NE-3MP), only the high-frequency part $f>180 \mathrm{kHz}$ of critical contribution to the ultrasonic spectra is obtainable from ultrasonic spectrometry. Hence, the verification of the characteristic relaxation rate $\Gamma$ from the ultrasonic measurement is quite difficult. In the Section "Strategies of verifying scaling function" (Sec. 5.1) it has been argued that there is no difference between the characteristic relaxation rate obtained from the dynamic light scattering and from the ultrasonic spectroscopy Eq.(5.1). Therefore, the determination of the characteristic relaxation rate $\Gamma_{0}(\varepsilon)$ from dynamic light scattering allows the reduced frequency $\Omega=2 \pi / \Gamma_{0} \varepsilon^{Z_{0} \tilde{v}}$ to be calculated and to be used in the calculations of the scaling function data.

\subsubsection{Scaling functions}

In a previous section it was supposed that the Bhattacharjee-Ferrell scaling function $F_{B F}(\Omega)$ fits best to the experimental ultrasonic measurement. In fact, this conclusion can be drawn only, when the other theoretical scaling functions, which have been proposed by Onuki or Folk and Moser (Section 4.3) are also considered. For this reason the experimental data have been evaluated with the aid of the quasi-universal empirical scaling function $F_{x}(\Omega)$, Eq.(4.23). A graphical representation in addition to the experimental data points for the systems ( $n$-PE-NM) and (NE-3MP) of the three scaling functions for 


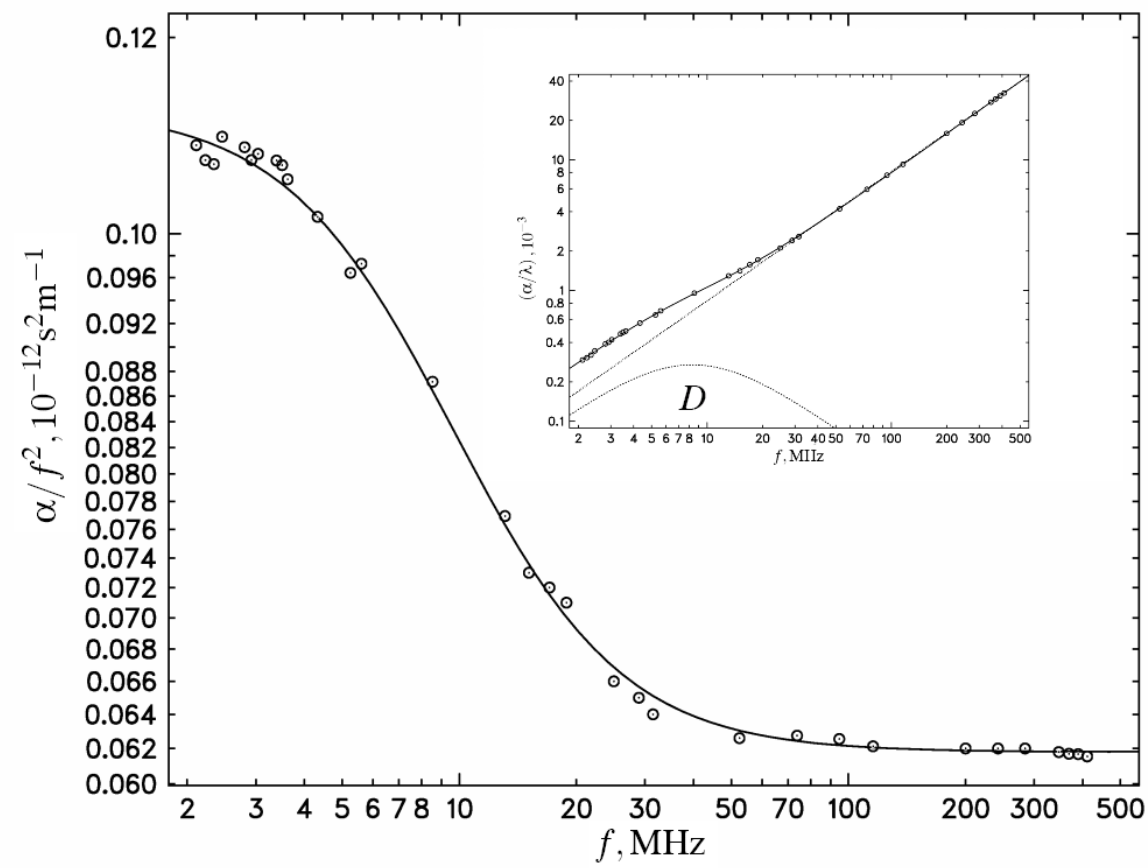

Figure 5.14: Ultrasonic attenuation spectrum in the format $\alpha / f^{2}$ versus $f$ of an (ETDOD) mixture at $25{ }^{\circ} \mathrm{C}$. The mole fraction of alcohol is $x_{e}=0.029$, corresponding with a mass fraction $Y_{e}=0.008$. The line is the graph of a Debye-type relaxation function with the following values of the parameters: $A_{D}=0.54 \cdot 10^{-3}$, $\tau_{D}=19.1 \mathrm{~ns}, B=79.2 \mathrm{ps}\left(B^{\prime}=\right.$ $\left.61.7 \cdot 10^{-15} \mathrm{~s}^{2} \mathrm{~m}^{-1}, c_{s}=1281.9 \mathrm{~ms}^{-1}\right)$.

$(x=B F, F M, O n$ in 4.23) is presented in Fig.(5.15). It is evident that the Onuki function is controlled by a half attenuation frequency that is distinctly larger than that of the empirical Bhattacharjee-Ferrell function. The Folk-Moser theory predicts a slope substantially different from the one of the Bhattacharjee-Ferrell function. In order to inspect the correspondence of the theoretical scaling function with the experimental facts more closely, the $F(\Omega)$ data for ( $n$-PE-NM) system and also for the (NE-3MP) system have been fitted to relation Eq.(4.23), treating $n_{x}$ and the half attenuation frequency $\Omega_{1 / 2}^{x}$ as adjustable parameters. Results of those investigations are presented in [76]. In Fig.(5.15), both series of measurements complement each other, showing that over a reduced frequency range of eight decades the $F_{B F}(\Omega)$ values fairly well fit to the scaling function of Bhattacharjee and Ferrell. The literature data of (NE-3MP) are closer to the value of $F(\Omega)=1$, caused by the higher upper limit of the measurement frequency range $(f \leq 17$ $\mathrm{MHz})$ as well as of the smaller than with $(n$-PE-NM) amplitude in the characteristic relaxation rate, $\Gamma_{0}=123 \cdot 10^{9} \mathrm{~s}^{-1}$. Consequently, the (NE-3MP) data extend to higher values 


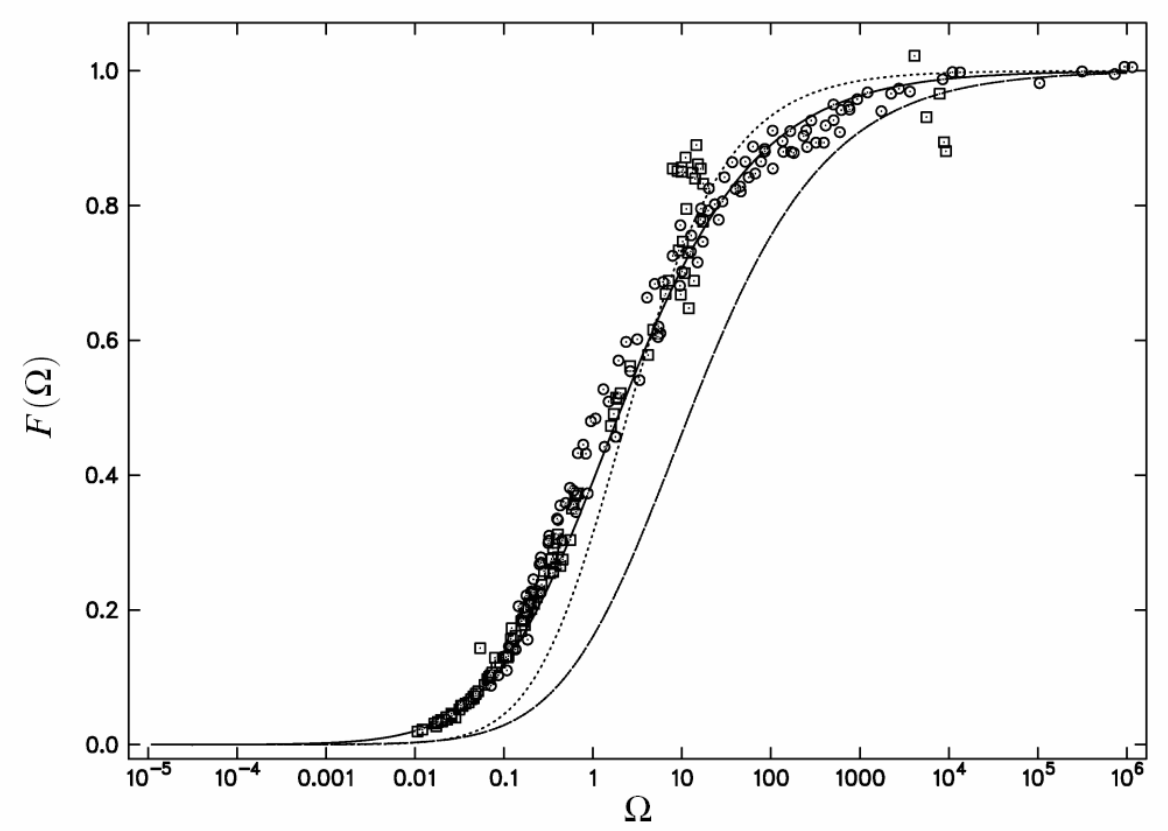

Figure 5.15: Scaling function data for the (n-PE-NM) mixture of critical composition, ( $\square$ ), and for the critical system (NE-3MP), ( $)$ ): The line is the graph of the empirical Bhattacharjee-Ferrell scaling function. The scaling function according to Onuki is shown by the dashed line, and that of the Folk-Moser by the dotted line. Also included are literature data [85].

of the reduced frequency $\Omega=2 \pi / \Gamma(\varepsilon)$. However, the central parameter in the empirical scaling function is the half attenuation frequency $\Omega_{1 / 2}$. This parameter can be derived from a regression analysis of experimental $F(\Omega)$ data in terms of the theoretical scaling functions $F_{x}(\Omega)$. This quantity can be used as an additional prove of the applicability of a scaling function. According to Eq.(4.24), a sensitive inspection of the shape of the three empirical functions $(x=B F, F M, O n)$ can be provided. In Fig.(5.16) the half attenuation frequency $\Omega_{1 / 2}^{B F}$, as following from Eq.(4.24) for the Bhattacharjee-Ferrell empirical scaling function versus scaled frequency $\Omega$ is plotted for two other critical mixtures. In Fig.(5.17) the same plots as in Fig.(5.16) but for the Folk-Moser, and the Onuki functions are shown. The overall increase in the $\Omega_{1 / 2}^{F M}$ values from 1 at $\Omega=0.1$ to about 5 at $\Omega=4000$ may be taken as an indication that both examples of critical mixtures (ET-DOD) as well as (ME-CH) do not fit as well to the shape of Folk-Moser scaling function as to that of the Bhattacharjee-Ferrell and the Onuki function. However, use of the parameter $n_{O n}$ in Eq.(4.24), yields values around 2, at variance with $\Omega_{1 / 2}^{O n}=6.2$. This result again 


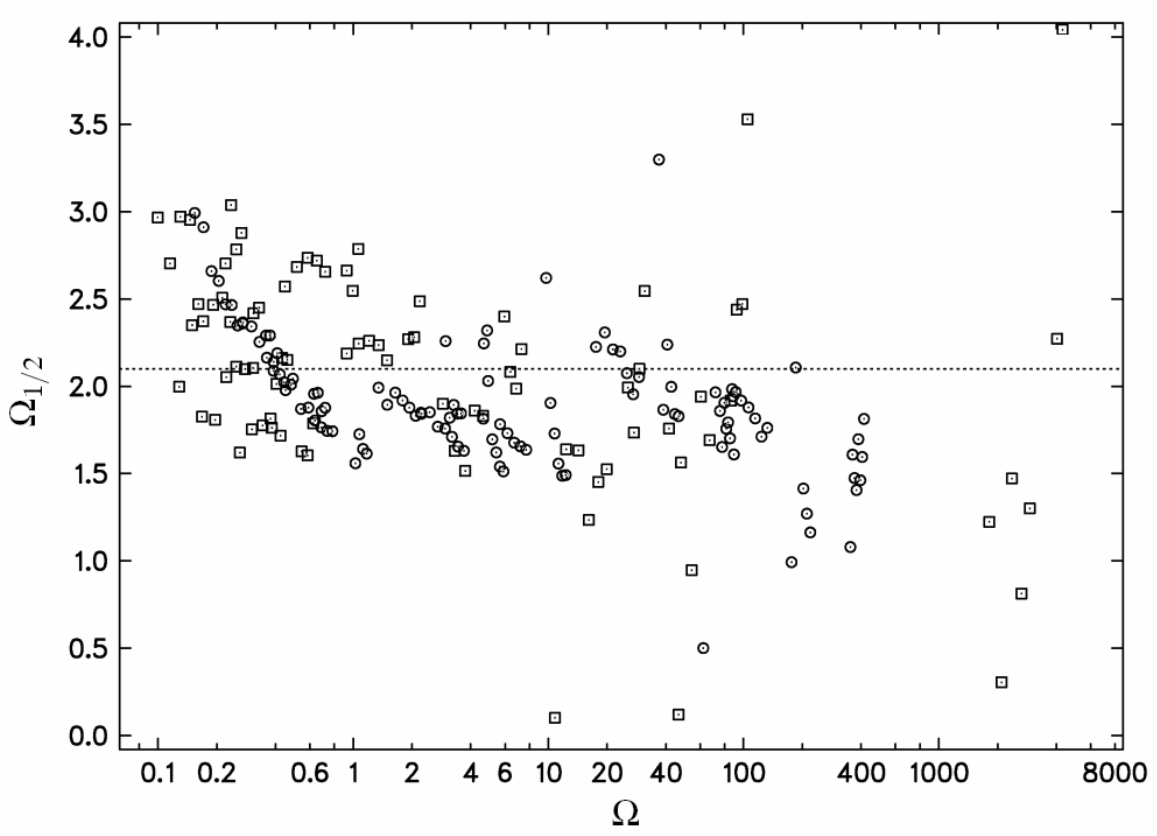

Figure 5.16: Half-attenuation frequency $\Omega_{1 / 2}^{x}$ for the Bhattacharjee-Ferrell empirical scaling function $(x=B F)$ plotted versus scaled frequency: (o) data for the system (ETDOD). ( $\square$ ) refers to (ME-CH) mixture of critical composition.

confirms the conclusion that the Bhattacharjee-Ferrell scaling function applies best to the experimental data. Results for most binary critical systems measured in present thesis indicate $\Omega_{1 / 2}^{B F}=2.1 \pm 0.1$. This value is in nice agreement with the theoretical prediction of Bhattacharjee and Ferrell. For the system ( $n$-PE-NM) the half-attenuation frequencies are somewhat smaller $\left(\Omega_{1 / 2}^{B F}=1.8\right)$. Previous investigations of the system (ET-DOD) had revealed a value $\left(\Omega_{1 / 2}^{B F}=1.2\right)$ [89]. However, if effects of hydrodynamic coupling are taken into account $\Omega_{1 / 2}^{B F}=2.1$ follows [99].

Another binary liquid, which has been studied within the series of mixtures without additional noncritical relaxation, was the system $(\mathrm{NE}-\mathrm{CH})$. This critical mixture, however, reveals an anomaly in the critical amplitude $S_{B F}$ Eq.(4.17) and in the adiabatic coupling constant $g$. As was mentioned previously, the assumption of the independency from temperature not fulfilled with these systems. If the critical amplitude is assumed constant, the data show systematic deviations from the theoretical $F_{B F}$ function in a way that most of them seem to be shifted to lower reduced frequency $\Omega$ (Fig.(5.18)). Since, the more recent 

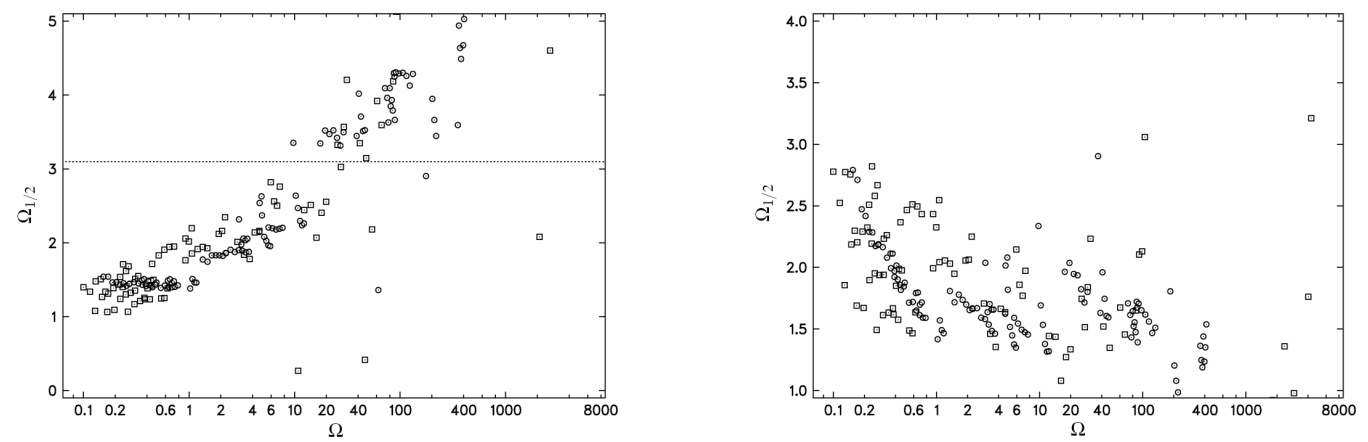

Figure 5.17: Left Figure: Half-attenuation frequency as in Fig.(5.16) but for the Folk-Moser scaling function $(x=F M)$. The dotted line represents the theoretical value $\Omega_{1 / 2}^{F M}=3.1$; Right Figure: Same plot but for the Onuki scaling function $(x=O n)$ with the theoretical value $\Omega_{1 / 2}^{O n}=6.2$.

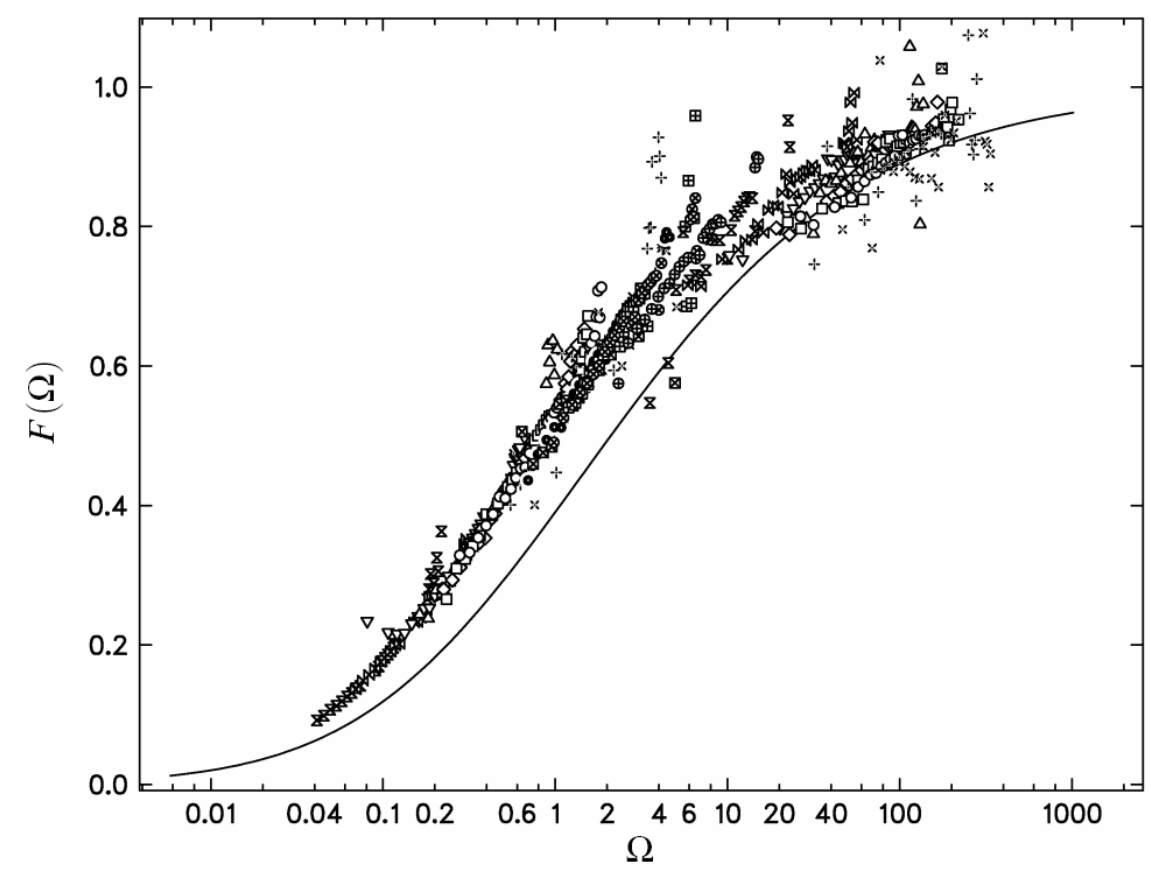

Figure 5.18: Scaling function data for the(NE-CH) system, with amplitude fixed at constant value: Figure symbols indicate different temperatures of measurement.

Folk-Moser and Onuki scaling functions are controlled by even larger half-attenuation fre- 
quencies, the discrepancy between experimental data and those functions would even be greater. When the amplitude is an adjustable parameter the experimental data fit nicely to the Bhattacharjee-Ferrell function with $\Omega_{1 / 2}^{B F}=2.1$ Fig.(5.18). Due to this fitting procedure, the critical amplitude $S_{B F}$ as well as the adiabatic coupling constant $g$ display a temperature dependence. This behavior of the critical amplitude contrasts other investigated systems without and with one additional chemical relaxations. The ultrasonic

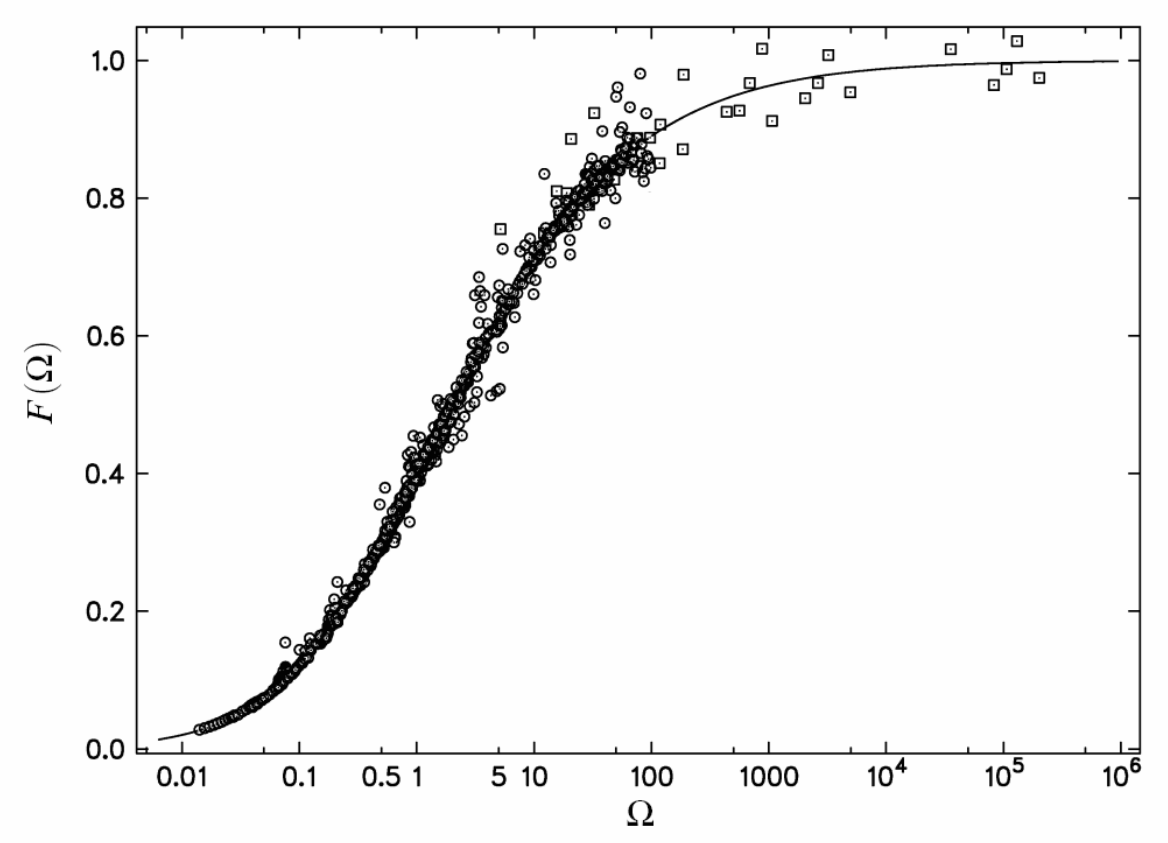

Figure 5.19: The same scaling function data as in Fig.(5.18), but with adjustable critical amplitude $S_{B F}$. All temperatures of Fig.(5.18) are represented by one symbol (o) and that from literature [84] by ( $\square)$.

attenuation data in Fig.(5.18) and Fig.(5.19) have been completed by literature data [84]. Both series of experimental scaling function data nicely complement each other, showing that, over a large reduce frequency range the $F(\Omega)$ values fairly fit to the BhattacharjeeFerrell scaling function, when the critical amplitude is considered an adjustable parameter.

Serious problems in the verification of the Bhattacharjee and Ferrell scaling function have appeared with the systems of (ME-HEX) and (ET-DOD). Firstly, these systems have been assumed to do not reveal a chemical relaxation. Hence, the first evaluation of experimental data have been considered from this point of view. As mentioned, however, in the previous section about ultrasonic measurements, an additional relaxation term has been 


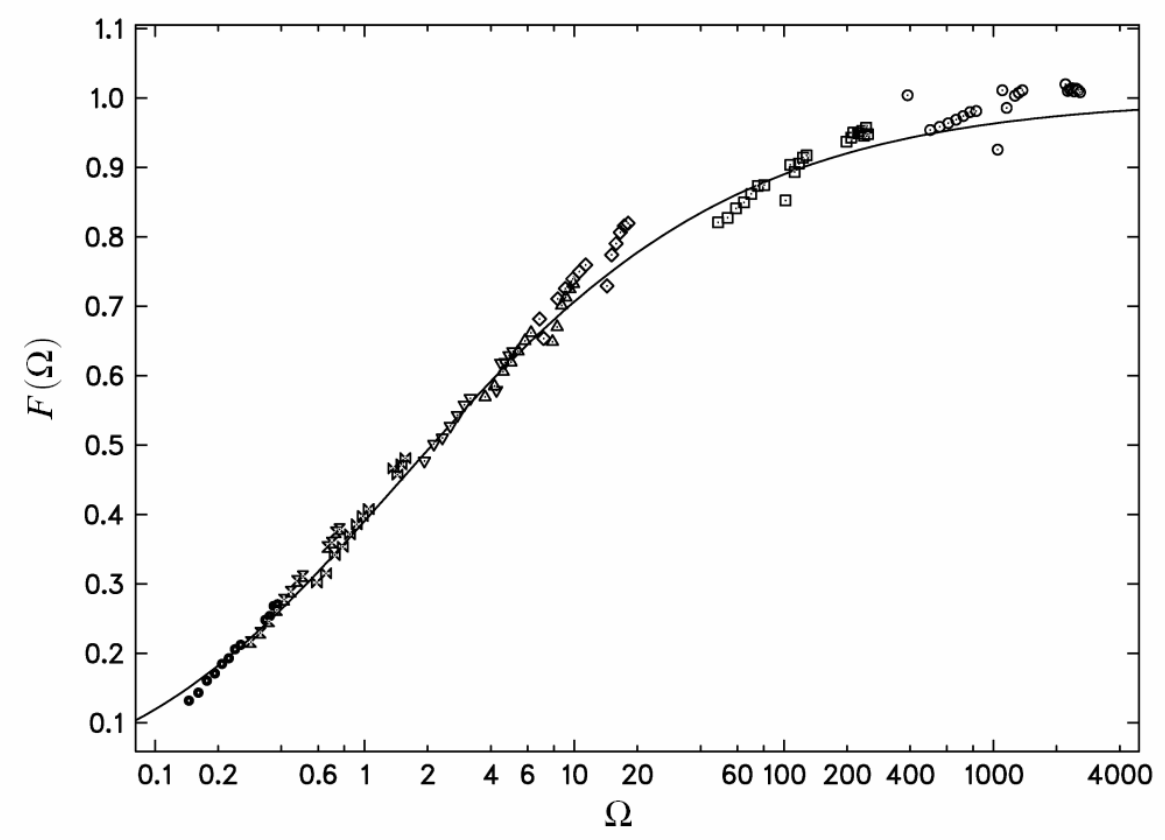

Figure 5.20: Scaling function data $F_{B F}$ of the binary mixture (ET-DOD) of critical contribution: without taken into account of the additional noncritical contribution.

found in both mixtures, with consequences for the data evaluation. In Fig.(5.20) the scaling function (ET-DOD) data, following on the assumption of a system without additional chemical relaxation are displayed. These data, follow the general trends in the scaling function but show systematic deviations and a large scatter. Moreover, the critical amplitude $S_{B F}$ as adjustable parameter similar to (NE-CH) demonstrates a dependence on temperature. However, taking into account the existence of an additional Debye-type relaxation term and considering its contribution to the low frequency wing in the spectra $f<1.7 \mathrm{MHz}$ according to Eq.(4.26), leads to an excellent agreement of the experimental scaling function with the theoretical form of the Bhattacharjee-Ferrell model. It also leads to independency of the critical amplitude on temperature. The same procedure has been successfully performed with the binary mixture (ME-HEX) [93] as well as with the system perfluoromethylcyclohexane-carbon tetrachloride [100]. Unfortunately, although recently performed measurements on a (NE-CH) mixture of noncritical composition point at an additional chemical relaxation. The unusual behavior of the critical amplitude of that system is still not clear. 


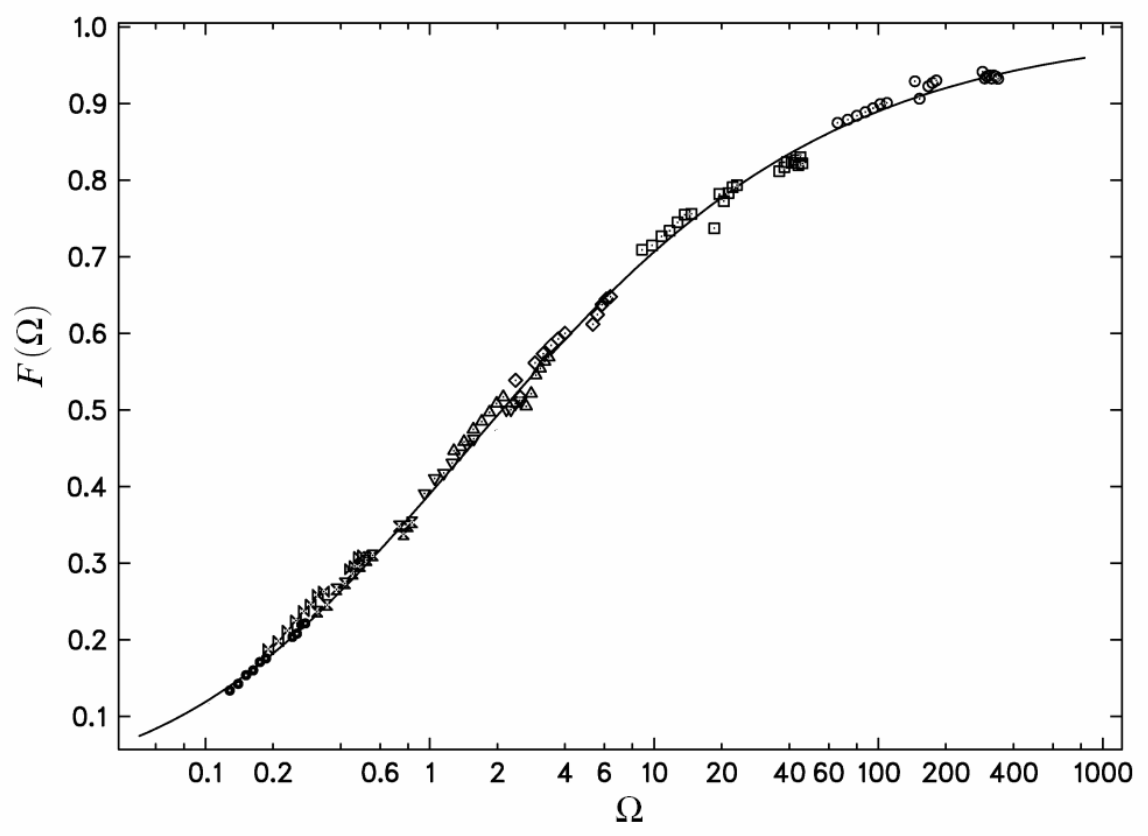

Figure 5.21: Same scaling function plot as in Fig.(5.20): but taking an additional Debye term into account Eq.(4.26).

\subsubsection{Amplitude $S_{B F}$ and coupling constant $g$}

The discussion of the quantities like the critical amplitude $S_{B F}$ as well as the coupling constant is interesting, because of their direct connection to thermodynamics. Furthermore, if heat capacity data as well as the fluctuation correlation length amplitudes $\xi_{0}$ are available, comparison of the amplitude values $S_{B F}$ and of the coupling constant $g$ from ultrasonic measurements with results from thermodynamic calculations is possible. From the amplitude parameter, $A(T)$, of the critical part in the ultrasonic spectra, the amplitude $S_{B F}$ can be derived, which according to Eq.(4.17) is related to the adiabatic coupling constant, $g$. The determination of the coupling constant will be presented using (ME-HEX) as an example.

Eq.(4.17) can be rewritten to yield the coupling constant $g$ as a function of $S_{B F}$ :

$$
g=\frac{C_{p b}}{\pi}\left[\frac{2 T_{c} S_{B F}}{\delta C_{p c} c_{s}\left(T_{c}\right)}\right]^{1 / 2}\left[\frac{2 \pi}{\Omega_{1 / 2} \Gamma_{0}}\right]^{\delta / 2}
$$


In this example the value of the critical amplitude $S_{B F}$ can be derived from the ultrasonic attenuation spectroscopy, using the expression:

$$
\left(\alpha / f^{2}\right)\left(T_{c}\right)=S_{B F} f^{-1.06}+\left(\alpha / f^{2}\right)_{b g}
$$

According to Eq.(5.16), the plot of the frequency normalized attenuation coefficient data versus $f^{-1.06}$ at low frequencies and at the reduced temperature $\varepsilon=1.6 \cdot 10^{-5}$ define a straight line with slope $S_{B F}=(0.88 \pm 0.02) \cdot 10^{-6} \mathrm{~s}^{-0.94} \mathrm{~m}^{-1}$ (see Fig.(4.1)). In Eq.(5.15) $C_{p b}$ as well as $C_{p c}$ are the background part and the amplitude of the singular part, respectively, of the heat capacity at constant pressure, if it is simply represented by the equation Eq.(5.4). Using $D=0$ and $E=0$ near $T_{c}, C_{p c}=A^{+} /\left(\alpha_{0} \rho\left(T_{c}\right)\right)=102.2 \mathrm{~J}$ / $(\mathrm{kgK})$, and $C_{p b}=2.34 \mathrm{~kJ} /(\mathrm{kgK})$ follows from the parameters of Eq.(5.4), where $\rho\left(T_{c}\right)=0.6841 \mathrm{~g} / \mathrm{cm}^{3}$. With $c_{s}\left(T_{c}\right)=1005.57 \mathrm{~m} / \mathrm{s}$ and $\Gamma_{0}=44 \times 10^{9} \mathrm{~s}^{-1}$, as mentioned before, $g=0.11 \pm 0.01$ follows from the amplitude $S_{B F}$ of the Bhattacharjee-Ferrell model. The adiabatic coupling constant can be alternatively derived from thermodynamic quantities, using Eq.(4.18). Therein $d T_{c} / d p$ is the pressure dependence of the critical temperature along the critical line and $\alpha_{p}$ is the thermal expansion coefficient at constant pressure at the critical point. The thermal expansion coefficient can be derived from the density data for the mixture of critical composition, following Eq.(4.19). The density data for the (ME-HEX) mixture, can be represented by the relation

$$
\rho=\rho_{c}+c_{1} \varepsilon+c_{2} \varepsilon^{1-\alpha_{o}}
$$

with $\rho_{c}=(0.68412 \pm 0.00001) \mathrm{g} / \mathrm{cm}^{3}, c_{1}=-(0.321 \pm 0.003) \mathrm{g} / \mathrm{cm}^{3}$, and $c_{2}=0$. With $\alpha_{p}^{c}=(1.35 \pm 0.1) \times 10^{-3} \mathrm{~K}^{-1}$ from this equation and $d T_{c} / d p=(32 \pm 1) \times 10^{-3} \mathrm{~K} / \mathrm{bar}$ [94] or $d T_{c} / d p=(33.8 \pm 1.2) \times 10^{-3} \mathrm{~K} /$ bar [95] $g=0.12 \pm 0.05$ results, in agreement with $g=0.11 \pm 0.01$ from the sonic attenuation coefficient measurements. In most systems which have been investigated in the present work the amplitude $S_{B F}$ shows only a weak dependence on frequency and temperature. Furthermore, from ultrasonic studies on systems without additional chemical relaxations the values obtained for the critical amplitude are in nice agreement with the results from thermodynamic calculations. For the binary critical systems listed in Table (5.9), $|g|$ values between 0.33 ( $n$-PE-NM), and 0.26 (NE-3MP) have been found. These values are small if compared to $|g|=0.7$ [96] and $|g|=0.98$ [97] as reported for the triethylamine/water critical mixture or to $|g|=1.3$ [98] and $|g|=2.1$ [101] as found for the critical systems ethylammonium nitrate/ $n$-octanol and isobutyric acid/water, respectively. The system with unusual amplitude, $S_{B F}$, and coupling constant is the critical mixture (NE-CH), as already mentioned before. The $S_{B F}$ values, obtained from fitting the experimental ultrasonic spectra to Eq.(5.14), display a 

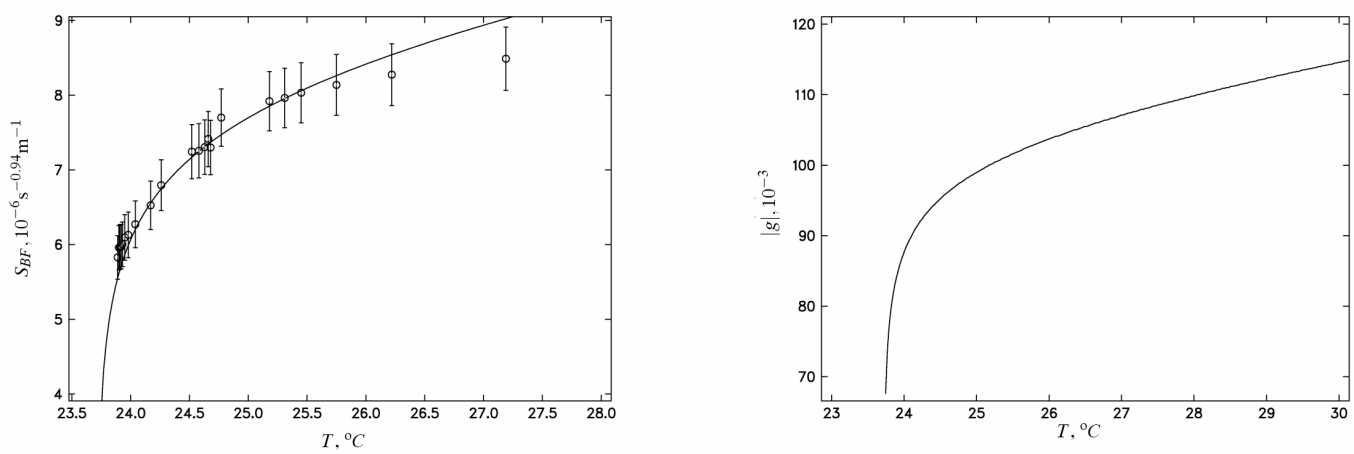

Figure 5.22: Left Figure: Amplitude $S_{B F}$ of the critical term in the ultrasonic attenuation spectra of the (NE-CH) system displayed as a function of temperature. The line is the graph of the theoretical relation Eq.(4.17) with the parameter values given in the text.; Right Figure: Amount $|g|$ of the adiabatic coupling constant of the (NE-CH) mixture of critical composition as a function of temperature $T$.

weak dependence upon temperature as shown by Fig.(5.22). This temperature dependence can be found in theoretical expression Eq.(4.16) if temperature dependencies in Eq.(5.4), where $C_{p b}=E^{+} \varepsilon+B^{+}$, and in the thermal expansion coefficient Eq.(4.19) are considered. With the aid of heat capacity data from the literature [79] and with thermal expansion coefficient as resulted from density measurements the agreement between temperature dependencies from theory and experiment is striking. The calculated coupling constant following from the fitting procedure in Fig.(5.22) shows a strong temperature behavior, too. It increases from $|g|=0.064$ near the critical point $T_{c}$ to $|g|=0.1$ at $\varepsilon=0.01$. Hence, the temperature variation in $S_{B F}$ is obviously due to a temperature dependence of the adiabatic coupling constant which results from a predominance of the temperature dependent thermal expansion coefficient in $g$. If this effect is disregarded the scaling function data from different runs do not fit to the Bhattacharjee-Ferrel scaling function, as was demonstrated in Fig.(5.18).

\subsection{Systems with complex background contributions}

As was mentioned in the Introduction, another aim of the investigations of critical binary systems with the aid of ultrasonic spectroscopy is to study the coupling between chemical relaxations and critical fluctuations in more complex systems. Especially, acoustic fields couple to the spatial Fourier components of the fluctuations, caused by their periodic variations of temperature. Hence, the question is whether the assumption of the additivity of critical contributions and the background contributions including noncritical relaxations, 
is correct. In this context three additional systems has been studied, isobutoxyethanolwater, 2,6-dimethylpyridine-water and triethylamine-water.

\subsubsection{Isobutoxyethanol-water}

Ultrasonic attenuation spectra of the isobutyric acid-water mixture of critical composition, reveal significant effects of slowing down near the critical temperature [101] not just in the monomer/linear dimer equilibrium of the carboxylic acid, but also in the linear dimer/cyclic dimer equilibrium, the latter being essentially an unimolecular process. The isobutoxyethanol-water mixture belongs to the class of micellar systems of non-ionic surfactants. In the past, conflicting results have been reported for this systems, namely, nonuniversal near-critical demixing properties and also universal behavior [102], [103], [104], [105]. Recent broadband ultrasonic studies of a variety of $C_{i} E_{i} /$ water systems [106], [107] suggested the idea of a fluctuation controlled monomer exchange [108], [109]. From the critical micelle mass fractions $Y_{c m c}$ of higher $\mathrm{C}_{i} \mathrm{E}_{i}$ homologues the critical micelle mass fraction $Y_{c m c}=0.07$ has been extrapolated for the system isobutoxyethanol/water $\left(i-\mathrm{C}_{4} \mathrm{E}_{1} / \mathrm{H}_{2} \mathrm{O}\right)$, corresponding with a critical micelle concentration $\mathrm{cmc}=0.6 \mathrm{~mol} / \mathrm{l}$ at room temperature [108], [109]. The $c m c$ of this short- chain amphiphile is, of course, not a sharply defined concentration but characterizes a transition range from predominantly molecularly dispersed solutions to mixtures containing micellar structures. For the system $\left(i-\mathrm{C}_{4} \mathrm{E}_{1} / \mathrm{H}_{2} \mathrm{O}\right)$ this transition regime is located well below the lower critical demixing mass fraction: $Y_{c}=0.318$ [117] and $Y_{c}=0.330$ [110] have been found, according to the equal-volume criterion. The critical temperature was determined visually as well as from measurements of half-power bandwith of an acoustical resonance at 4000 $\mathrm{kHz}$ of a biplanar resonator, Fig.(5.23), and was $T_{c}=298.10 \mathrm{~K}$. This value is smaller than the critical temperature in previous experimental studies, $T_{c}=299.60 \mathrm{~K}$ [117]. Hence micelles and concentration fluctuations exist simultaneously near the critical demixing point. The question whether or not the fluctuations in the local concentration interfere with the micelle formation/decay kinetics has not been answered so far [111]. A broadband ultrasonic spectrometry study of the $\left(i-\mathrm{C}_{4} \mathrm{E}_{1} / \mathrm{H}_{2} \mathrm{O}\right)$ system has been performed recently [110] in order to investigate the aggregation kinetics as well as the critical dynamics at the lower demixing point. 


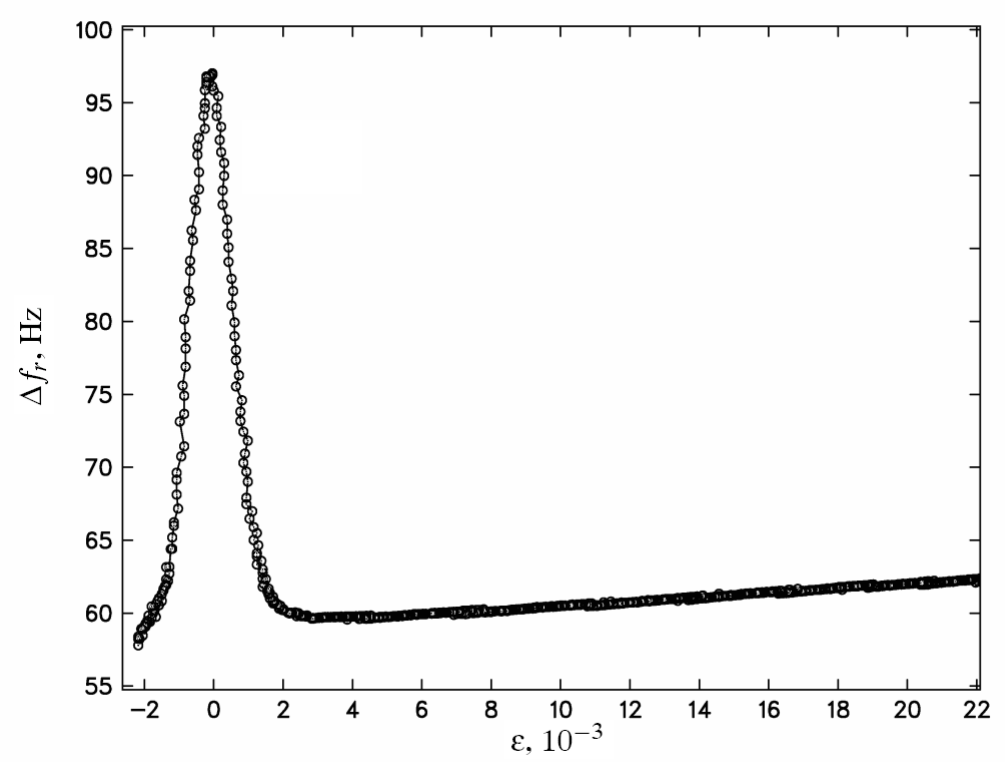

Figure 5.23: Half-power bandwidth $\Delta f_{r}$ of an acoustical resonance at $4000 \mathrm{kHz}$ of a cylindrical biplanar resonator filled with the $\left(i-\mathrm{C}_{4} \mathbf{E}_{1} / \mathrm{H}_{2} \mathrm{O}\right)$ mixture of critical composition, plotted versus reduced temperature $\varepsilon$.

The relaxation rate $\Gamma$ of concentration fluctuations, derived from the critical contribution to the ultrasonic spectra, did not follow power law Fig.(5.24) as did the $\Gamma$ values from static and dynamic light scattering [112]. Therefore, additional measurements of low frequency part of the ultrasonic spectra have been performed between $200 \mathrm{kHz}$ and $3 \mathrm{MHz}$ and have been evaluated on the assumption that the relaxation rate of critical fluctuations in ultrasonic spectra is governed by the relaxation rate $\Gamma_{D L S}$ from the dynamic light scattering. An example of an ultrasonic excess attenua-

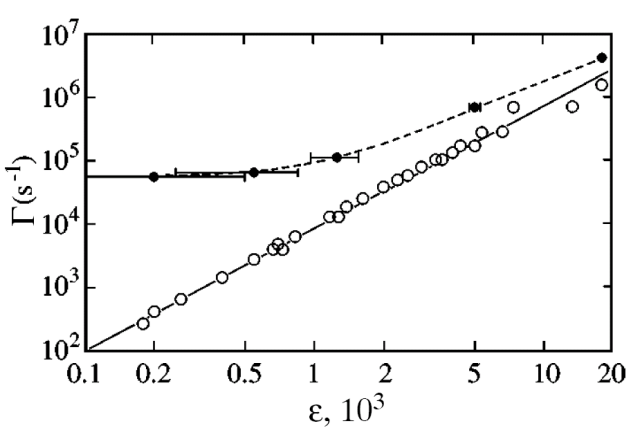

Figure 5.24: Relaxation rate $\Gamma_{D L S},(\circ)$, and non-universal behavior of $\Gamma_{U S},(\bullet)$, near critical point. tion spectrum of the $\left(i-\mathrm{C}_{4} \mathrm{E}_{1} / \mathrm{H}_{2} \mathrm{O}\right)$ mixture of critical composition at $25{ }^{\circ} \mathrm{C}$ is displayed in Fig.(5.25). A careful analysis of the broadband sonic $\left(i-\mathrm{C}_{4} \mathrm{E}_{1} / \mathrm{H}_{2} \mathrm{O}\right)$ spectra at $Y_{c}$ lead to the conclusion that at least three relaxation terms,

$$
(\alpha \lambda)_{e x c}^{*}=R_{c}(f)+R_{H}^{\#}(f)+R_{D}(f)
$$




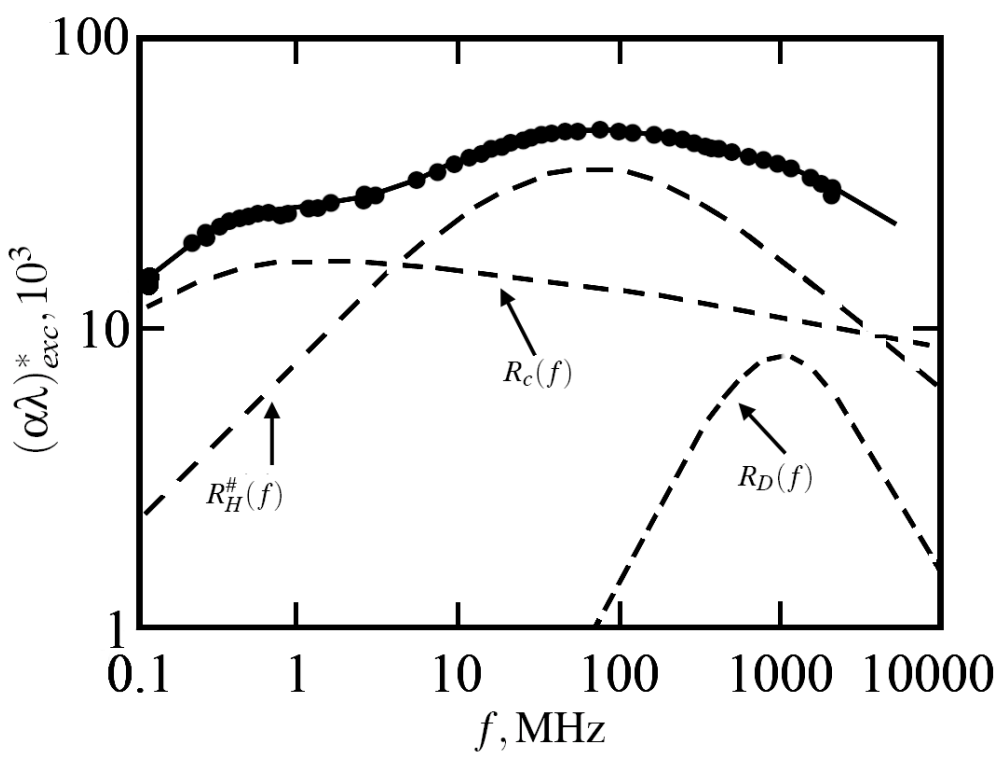

Figure 5.25: Acoustical attenuation-per-wavelength spectrum for $\left(i-\mathrm{C}_{4} \mathbf{E}_{1} / \mathrm{H}_{2} \mathrm{O}\right) \mathrm{mix}$ ture of critical composition at $25{ }^{\circ} \mathbf{C}$. The subdivision follows from nonlinear least-squares regression analysis of the experimental spectrum of terms of Eqs.(5.18) and (3.31).

are required for an adequate analytical representation of the experimental data [110]. In addition to a broad critical contribution $R_{c}(f)$ Eq.(4.16), a restricted Hill-type $R_{H}^{\#}(f)$ Eq.(3.39), and a Debye-type $R_{D}(f)$, Eq.(3.32), relaxations are indicated by relative maxima in the spectrum, Fig.(5.25). Here the restricted Hill term reflects the micelle formation and decay kinetics [113]. The additional contribution represented by the Debye term exists already in $i-\mathrm{C}_{4} \mathrm{E}_{1}$ without water added. It seems to reflect a chemical equilibrium of $i-\mathrm{C}_{4} \mathrm{E}_{1}$ molecules, likely a step in the isobutoxyethanol isomerization scheme. Information about the spectrum has been achieved from broadband measurements at noncritical mixtures with $0.09 \leq Y<1$ in [113]. This complicated spectrum requires a special regression analysis. In order to reduce the number of adjustable parameters, ones more, the critical relaxation rate $\Gamma(\varepsilon)$ from light scattering has been used as known parameter in the regression analysis of the spectra. Furthermore, in the fitting procedure of the low-frequency measurements (inset of Fig.(5.26)) between $200 \mathrm{kHz}$ and $3 \mathrm{MHz}$ the parameters of the Hill and Debye relaxation terms have been obtained by interpolation and extrapolation from previous data [110]. Therefore, in determining the scaling function $F(\Omega)$ (Fig.5.26), the only adjustable parameter in the data evaluation was the critical amplitude $S_{B F}$. As result, the temperature dependence in $S_{B F}$, as follows from the fitting procedure of the attenuation coefficient data in terms of the Bhattacharjee-Ferrell scaling function, is almost as large as for the (NE-CH) system. According to Eq.(5.15), 


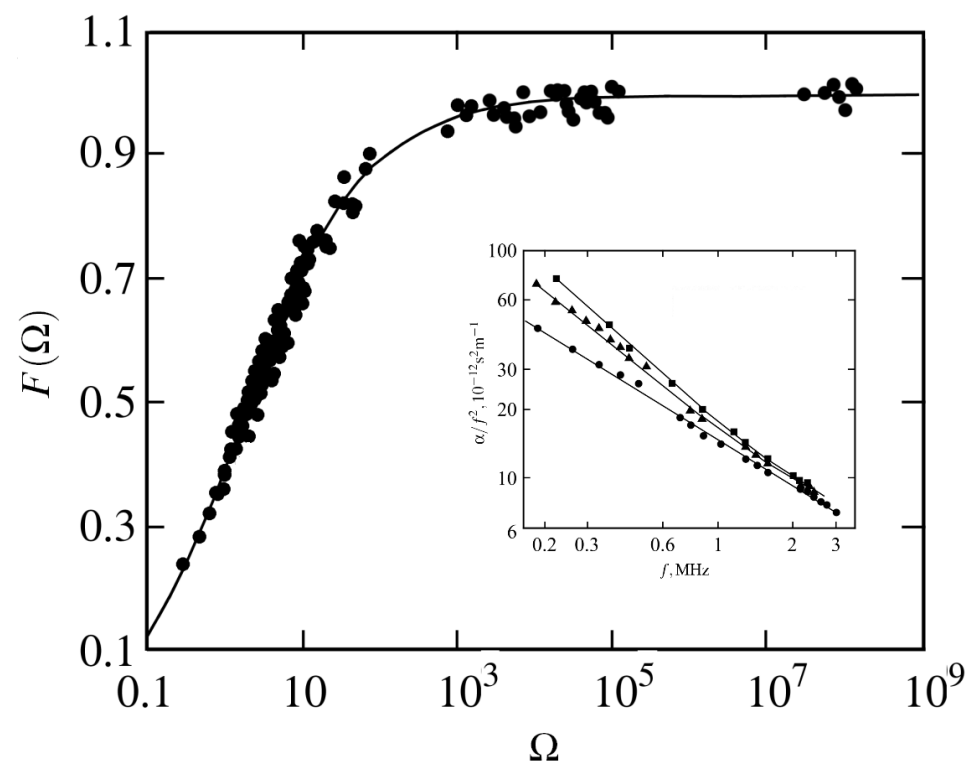

Figure 5.26: Scaling function data for the $\left(i-\mathrm{C}_{4} \mathbf{E}_{1} / \mathbf{H}_{2} \mathrm{O}\right.$ system.) The line is the graph of the of the Bhattacharjee-Ferrell scaling function Eq.(4.22) with $\Omega_{1 / 2}=2.1$. The inset shows the low-frequency part of frequency normalized acoustical attenuation spectra. (•) $20.20^{\circ} \mathrm{C}$, (ム) $23.64{ }^{\circ} \mathrm{C},(\boldsymbol{\square}) 24.95{ }^{\circ} \mathrm{C}$.

using $C_{p c}=55.45 \mathrm{~J} / \mathrm{kgK}$ and $C_{p b}=3740 \mathrm{~J} / \mathrm{kgK}$ [114], the $g$ values from the amplitudes $S_{B F}$ decrease from $g=1.77$ at $\varepsilon=0.0012$ to $g=1.33$ at $\varepsilon=0.019$. In contrast, almost constant $g=1.35$ results from the thermodynamic relation Eq.(4.18). In deriving this value $d T_{c} / d P=39.8 \cdot 10^{-3} \mathrm{~K} / \mathrm{bar}$ [115] has been used and $\rho\left(T_{c}\right)=0.9715 \mathrm{~g} / \mathrm{cm}^{3}$ [110]. However, when $\Gamma(\varepsilon)$ is fixed and using coupling constant $g$ from thermodynamical calculations, according to Eq.(5.15) allows for an excellent representation of the experimental data on one master curve, as presented in Fig.(5.26).

\subsubsection{2,6-dimethylpyridine-water}

Another binary critical mixture which has been investigated in the framework of systems with complex background contributions was 2,6-dimethylpyridine-water. The mixture (2,6-DMP- $\left.\mathrm{H}_{2} \mathrm{O}\right)$ displays a lower critical demixing point at $T_{c}=306.83 \mathrm{~K}$ with critical mass fraction $Y_{c}=0.291$ of 2,6-DMP . Moreover, as 2,6-DMP is a weak base, protolysis may take place in aqueous solutions:

$$
2,6-\mathrm{DMP}+\mathrm{H}_{2} \mathrm{O} \rightleftarrows 2,6-\mathrm{DMPH}^{+}+\mathrm{OH}^{-},
$$

and, by analogy with the stacking of cyclic purine bases, the formation of 2,6-DMP ag- 
gregates may be suggested [121]. A protolysis term also exists in the spectrum of the mixture of noncritical composition $\left(x=0.148, \tau_{1}=2.7 \mathrm{~ns}, A_{1}=2.1 \cdot 10^{-3}, 25^{\circ} \mathrm{C}\right)$, shown in Fig.(5.27).

Dielectric and ultrasonic relaxation measurement as well as depolarized Rayleigh scattering and nmr studies reported in [118], [119], [120], have supported the idea of 2,6-DMP aggregates including water. In the previous treatment of $\left(2,6-\mathrm{DMP}-\mathrm{H}_{2} \mathrm{O}\right)$ spectra [120] two Debye-type relaxation terms have been considered in addition to the critical contribution. The recent evaluation proved one noncritical relaxation term $R_{D}(f)$ to be sufficient for an adequate representation of the experimental data Fig.(5.28), which likely reflects the pro-

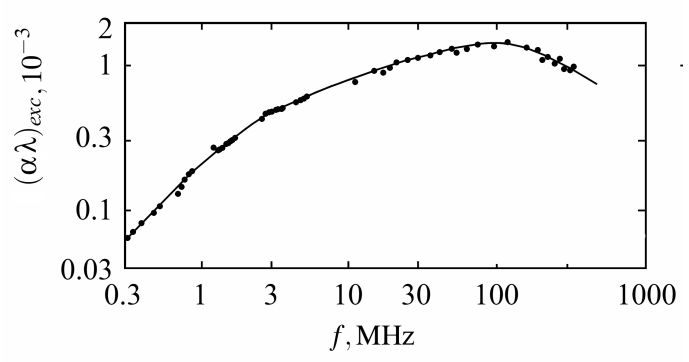

Figure 5.27: Ultrasonic excess attenuation spectrum for 2,6dimethylpyridine-water mixture of noncritical composition $Y=$ 0.148 at $25^{\circ} \mathbf{C}$.

tolysis equilibrium Eq.(5.19). Hence, at least two relaxation terms,

$$
(\alpha \lambda)_{e x c}^{*}=R_{c}(f)+R_{D}(f)
$$

are required for an adequate analytical representation of the experimental data [123]. An-

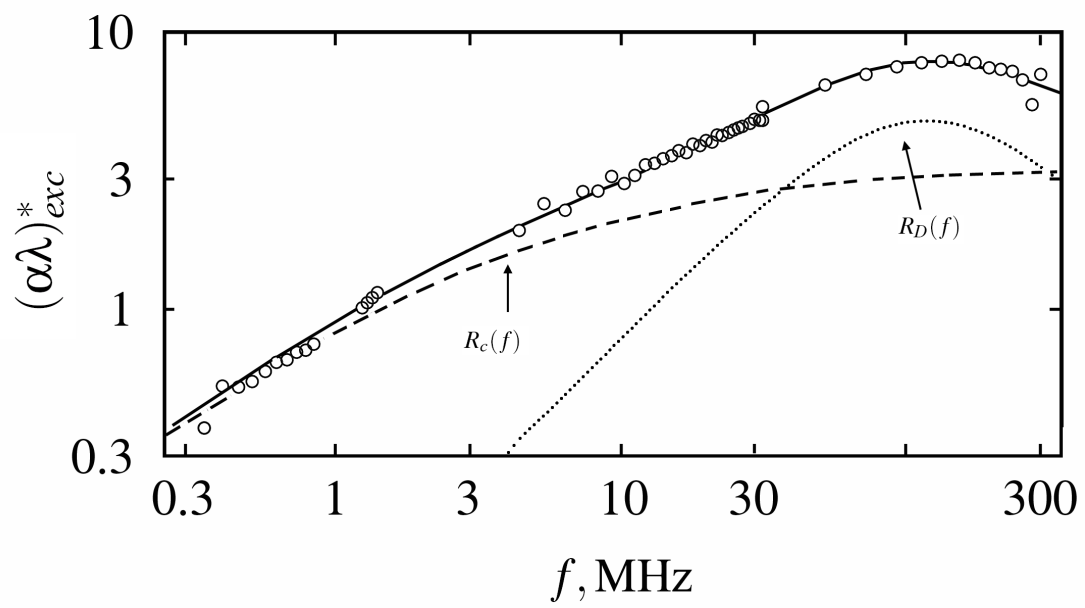

Figure 5.28: Excess-attenuation-per-wavelength spectrum for the 2,6-dimethylpyridinewater mixture of critical composition at $30{ }^{\circ} \mathbf{C}$. Dashed and dotted lines are graphs of the critical $R_{c}(f)$ and noncritical terms $R_{D}(f)$, respectively, in the spectrum Eq.(5.20). The full line shows the sum of these terms. 


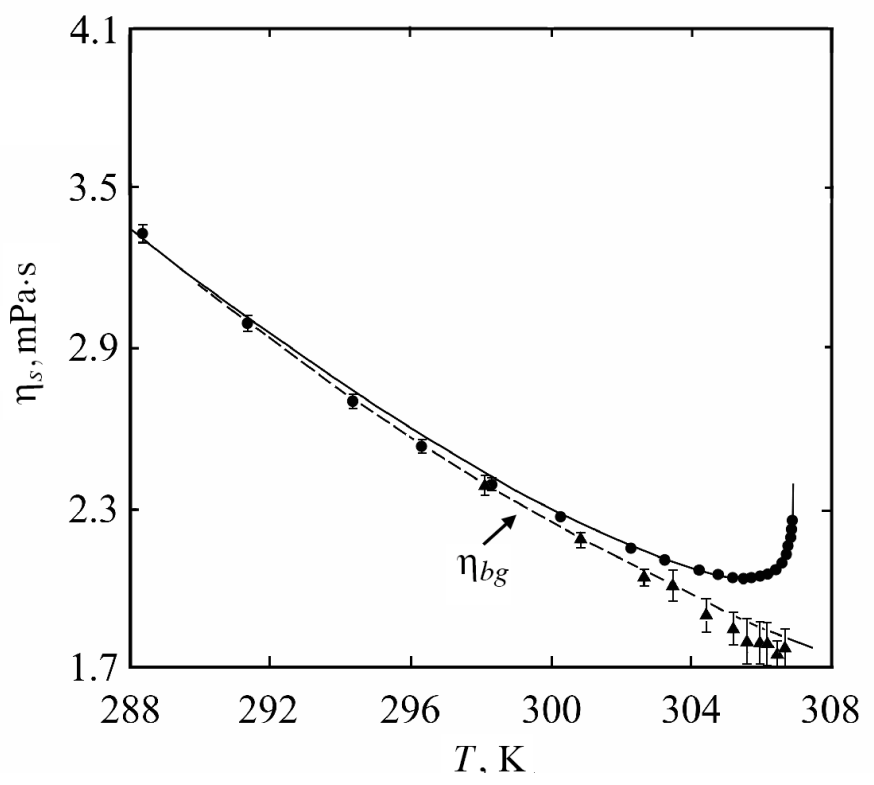

Figure 5.29: Shear viscosity data of 2,6-dimethylpyridine-water from capillary viscosimeter measurements $(\bullet)$ and from the extrapolation of high-frequency viscosity data $(\Delta)$ : Also shown are the graphs of the viscosity function (full line) and of the noncritical background part in the viscosity (dashed line).

other advantage of the (2,6-DMP- $\left.\mathrm{H}_{2} \mathrm{O}\right)$ system is the availability of recent specific heat, shear viscosity, and dynamic light scattering data [116], which facilitate the evaluation of the critical part in the ultrasonic attenuation spectra. In Fig.(5.29) the shear viscosity data are displayed along with the graph of Eq.(4.31) to show that the experimental $\eta_{s}$ values are well represented by theory. Also given by the dashed line is the graph of the background part $\eta_{b g}$. In addition to the shear viscosity data from the capillary viscosimeter measurements, high-frequency viscosity data obtained from the impedance spectrometer (Sec.(3.4) are presented in Fig.(5.29) [118]). The combined evaluation of the high frequency shear viscosity and the dynamic light scattering data yields the background contribution $\eta_{b g}$, in nice agreement with extrapolated high frequency viscosities $\eta_{s}(0)=\lim _{f \rightarrow 0} \eta_{s}(f)$, measured between 5 and $120 \mathrm{MHz}$ [116] where the critical contribution are supposed to be fade out. This is another indication that the crossover formalism describes data correctly.

Knowledge of the noncritical background contributions $R_{D}(f)=A_{D} \omega \tau_{D} /\left(1+\omega^{2} \tau_{D}^{2}\right)$ Eq.(5.20) and $B$ in Eq.(3.31) in the ultrasonic spectra allows the scaling function $F(\Omega)$ to be calculated without any adjustable parameter. Using the characteristic relaxation rate $\Gamma(\varepsilon)$ and the especially measured resonator data the (Eq.(5.14)) has been applied with $A_{D}(T), \tau_{D 1}(T)$, and $B(T)$ data as simply obtained by interpolation of the values given in Table (5.4). The values for characteristic relaxation rate, viscosity parameter, as well 


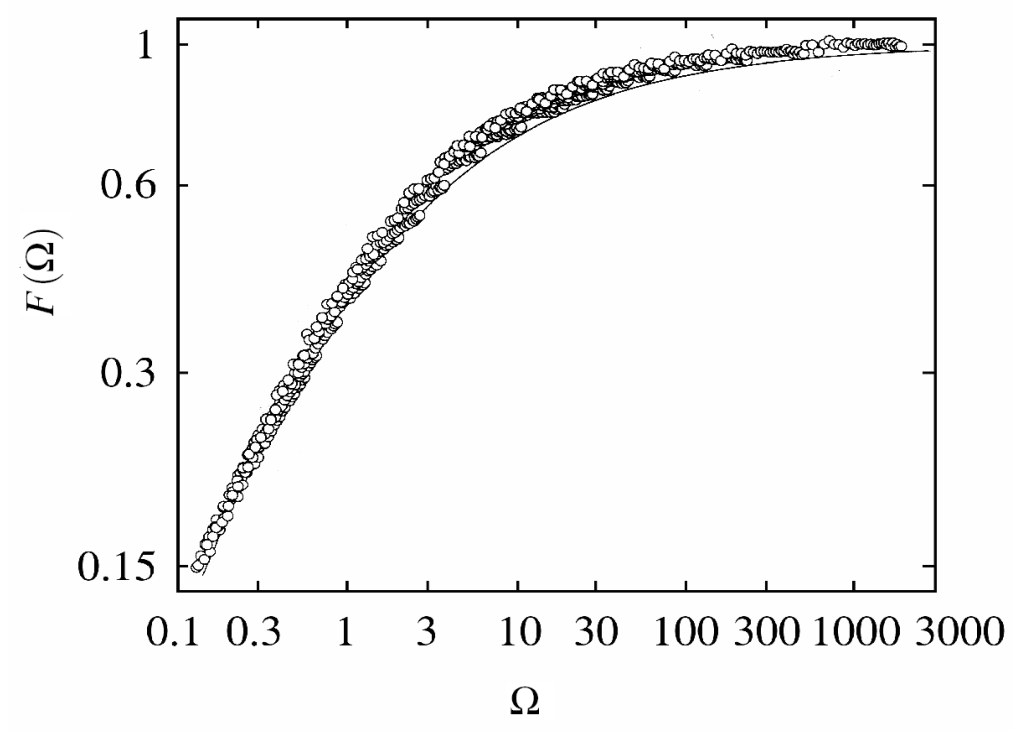

Figure 5.30: Scaling function data for the 2,6-DMP/ $/ \mathrm{H}_{2} \mathrm{O}$ mixture of critical composition as determined using relaxation rates from the shear viscosity and dynamic light scattering measurements with $\left.\Gamma_{0}=25 \cdot 10^{9} \mathbf{s}^{-1}\right)$ : . The line is the graph of the empirical scaling function of the Bhattacharjee-Ferrell theory with $\Omega_{1 / 2}=2.1$ ).

as critical amplitude and the coupling constant can be found in Table (5.9). The scaling function with $\Omega_{1 / 2}=2.1$ is presented in Fig.(5.30). The data, measured at different frequencies and temperatures, clearly define one master curve and almost agree also with the theoretically predicted function. This result, following from an evaluation without any unknown parameter, may be taken to indicate the consistency of the theoretical models.

\begin{tabular}{cccc}
\hline \hline$T_{c}-T \mathrm{~K} A_{D}\left(10^{-3}\right)$ & $\begin{array}{c}\tau_{D}(\mathrm{~ns}) \\
\pm 0.4\end{array}$ & $\begin{array}{c}B(\mathrm{ps}) \\
\pm 0.1\end{array}$ & \pm 1 \\
\hline 8.40 & 9.6 & 1.3 & 70.3 \\
2.40 & 9.6 & 1.3 & 70.4 \\
1.40 & 8.4 & 1.5 & 65.8 \\
0.80 & 9.6 & 1.4 & 63.5 \\
0.20 & 12.1 & 1.3 & 62.0 \\
0.02 & 12.1 & 1.3 & 62.0 \\
\hline \hline
\end{tabular}

Table 5.4: Noncritical parameters in the ultrasonic spectra of the $\left(2,6-\mathrm{DMP} / \mathrm{H}_{2} \mathrm{O}\right) \mathrm{mix}-$ ture of critical composition. 


\subsubsection{Triethylamine-water}

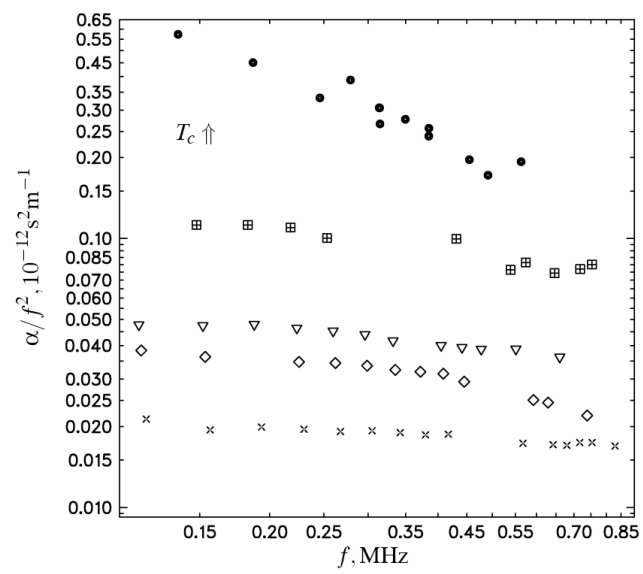

Figure 5.31: Low frequency part of the ultrasonic spectra (TEA$\mathrm{H}_{2} \mathrm{O}$ ) mixture of critical composition, $\left(\mathbf{R U N}_{2}\right)$ 2: $\mathrm{x}, T=283.92 \mathrm{~K}$; $\diamond, T=289.80 \mathrm{~K} ; \nabla, T=287.76 \mathrm{~K}$; 田 $T=289.79 \mathrm{~K} ; \bullet, T \approx 291.36 \mathrm{~K}$ $=T_{c}$.
Another system in the studies of critical mixtures with complex background contributions was triethylamine-water (TEA- $\left.\mathrm{H}_{2} \mathrm{O}\right)$. The critical mixture (TEA- $\mathrm{H}_{2} \mathrm{O}$ ) had been investigated in many previous studies with ultrasonic methods as well as with light scattering methods. Unfortunately, in those studies [124], [125] the scaling function data did not fall on one curve, and additionally, the data deviated substantially from the theoretical form. Furthermore, the amplitude of the characteristic relaxation rate $\left(\Gamma_{0}=45 \cdot 10^{9} \mathrm{~s}^{-1}\right)$ obtained from ultrasonic measurements was distinctly smaller than that obtained from light scattering data and shear viscosity measurements $\left(\Gamma_{0}=96\right.$. $\left.10^{9} \mathrm{~s}^{-1}\right)$. Therefore, low frequency ultrasonic measurements, Fig.(5.31) have been performed at critical composition (the mass fraction of amine $Y_{c}=0.321$, critical temperature $T_{c}=$

$18.21{ }^{\circ} \mathrm{C}$ ) and additionally, the shear viscosity data as well as the light scattering data have been reevaluated within the framework of the crossover formalism. In Fig.(5.32), the resulting plots of the characteristic relaxation rate and the shear viscosity are presented. Furthermore, the measured viscosity as well as the derived mutual diffusion coefficient have been compared with literature data [131] at $15^{\circ} \mathrm{C}$, Fig.(5.33). The diffusion coefficient versus mole fraction plot of $\left(\mathrm{TEA}-\mathrm{H}_{2} \mathrm{O}\right)$ and the viscosity versus mole fraction plot of $\left(\mathrm{TEA}-\mathrm{H}_{2} \mathrm{O}\right)$, both agree nicely with literature data. In previous studies the experimental data, had not been treated in terms of modern crossover theories. Hence, the investigation in the present thesis has been focused on the low frequency part Fig.(5.31) of the ultrasonic spectra of $\left(\mathrm{TEA}-\mathrm{H}_{2} \mathrm{O}\right)$ as well as the treatment of the data within the framework of crossover formalism Eq.(4.31). Additionally, the noncritical contribution to the ultrasonic spectra, and its coupling to the critical fluctuations has been studied extensively. More recent broadband ultrasonic attenuation spectrometry revealed two noncritical background relaxation terms, in addition to the always existing asymptotic high frequency background term [97]. In Fig.(5.34) the ultrasonic attenuation spectrum for the (TEA- $\mathrm{H}_{2} \mathrm{O}$ ) mixture of critical composition is displayed at $17^{\circ} \mathrm{C}$ and $15^{\circ} \mathrm{C}$. The finding of the broadband (TEA- $\mathrm{H}_{2} \mathrm{O}$ ) spectra to be composed of different contributions is indicated by dashed lines. Careful analysis of the experimental data [97] has revealed the existence of two Debye type relaxation terms $\left(R_{D_{1}}^{+}, R_{D_{2}}^{+}\right)$in addition to the critical term 

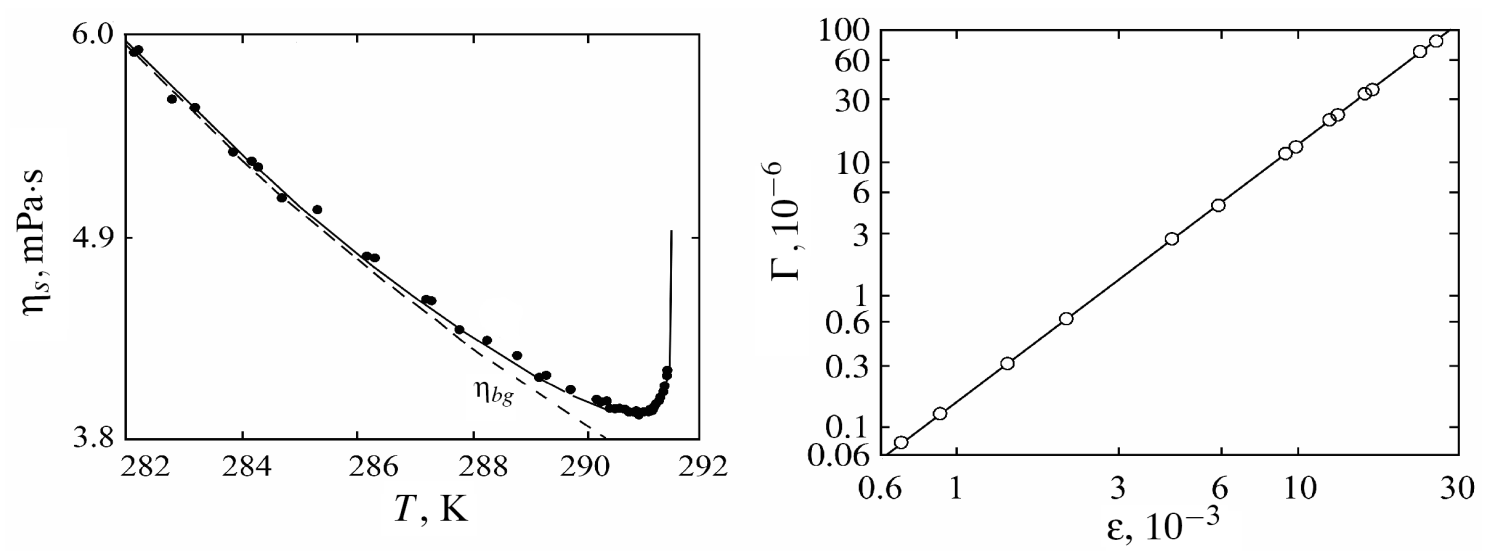

Figure 5.32: Left Figure: Shear viscosity $\eta_{s}$ of the (TEA- $\left.\mathrm{H}_{2} \mathrm{O}\right)$ mixture of critical composition versus temperature $T$. The full line shows the viscosity function, the dashed line represents the background part $\eta_{b g}$; Right Figure: Relaxation rate $\Gamma$ of order parameter fluctuations of the (TEA- $\mathrm{H}_{2} \mathrm{O}$ ) mixture of critical composition as a function of reduced temperature $\varepsilon$. Symbols represent data as obtained from the combined evaluation of shear viscosity and dynamic light scattering results. The line is the graph of the power law $\Gamma=\Gamma_{0} \varepsilon^{Z_{0} \tilde{v}}$ with the theoretical critical exponent $Z_{0} \widetilde{v}=1.903$ and the amplitude $\Gamma_{0}=96 \cdot 10^{9} \mathrm{~s}^{-1}$.
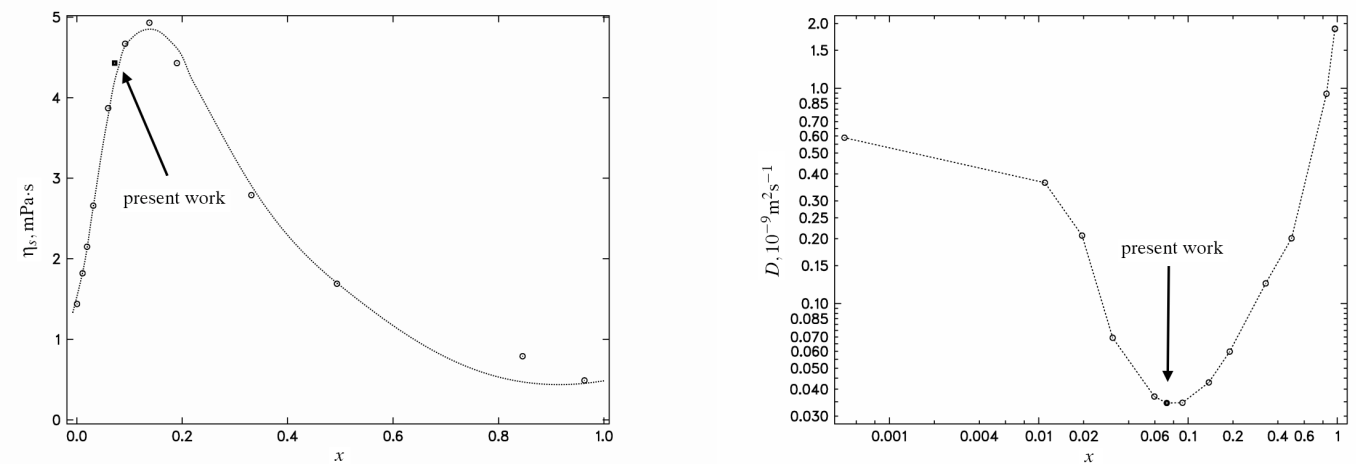

Figure 5.33: Left Figure: Shear viscosity $\eta_{s}$ versus mole fraction of $\left(\mathrm{TEA}-\mathrm{H}_{2} \mathrm{O}\right)$ at $15{ }^{\circ} \mathrm{C}$ Right Figure: Diffusion coefficient $D$ versus mole fraction of $\left(\mathrm{TEA}-\mathrm{H}_{2} \mathrm{O}\right)$ at $15^{\circ} \mathrm{C},[131]$, full symbols denote values from present work.

and the frequency independent contribution $B^{\prime}$. Triethylamine is a strong base. The high frequency Debye relaxation term $R_{D_{1}}^{+}$has been assigned [97] to the protolysis reaction:

$$
\mathrm{TEA}+\mathrm{H}_{2} \mathrm{O} \leftrightharpoons \mathrm{TEAH}^{+}+\mathrm{OH}^{-}
$$

with a relaxation time between 1 and $1.7 \mathrm{~ns}$. The low-frequency Debye term $R_{D_{2}}^{+}$has been 


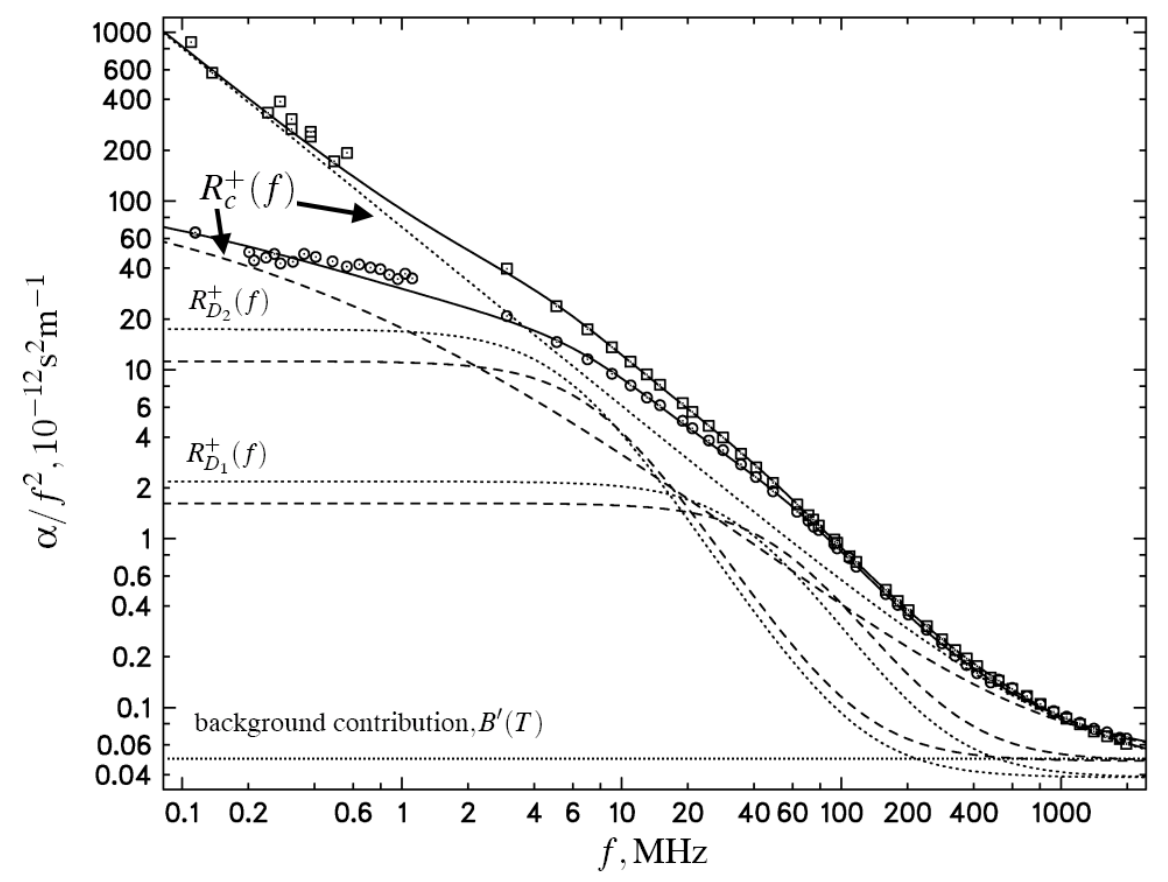

Figure 5.34: Ultrasonic attenuation coefficient per $f^{2}$ versus frequency $f$ for the (TEA$\mathrm{H}_{2} \mathrm{O}$ ) mixture of critical composition at $18.00{ }^{\circ} \square \mathrm{C}$, and $15.00{ }^{\circ} \mathrm{C} \circ$. The subdivision of the spectrum into a critical part (" $R_{c}(f)$ ") and noncritical background contributions $\left(" B^{\prime},, R_{D_{1}}^{+}(f),, " R_{D_{2}}^{+}(f)\right.$ ") is indicated by dashed and dotted lines. The full lines are in the graph the complete spectral functions.

related to rotational isomerization of the ethyl groups:

$$
\mathrm{TEA} \leftrightharpoons \mathrm{TEA}^{*}
$$

with TEA* denoting a structural conformer of TEA. Rotational isomerization has been assumed to be reflected by acoustical relaxation in pure triethylamine, [126], with relaxation time on the order of 2 ns. Both Debye relaxation terms have to been taken into account in the evaluation of the scaling function. Assuming the Bhattacharjee-Ferrell theory to apply to the critical part $R_{c}^{+}(f)$ in the sonic attenuation coefficient the ultrasonic spectra have been analytically represented by function Eq.(4.26) with the relaxation rate $\left(\Gamma_{0}=96 \cdot 10^{9} \mathrm{~s}^{-1}\right)$ from light scattering and viscosity measurements, taking into account the crossover formalism. Assuming in the temperature range between $10.7^{\circ} \mathrm{C}$ and $T_{C}=18.21{ }^{\circ} \mathrm{C}$, the critical amplitude $S_{B F}$ Eq.(4.17) to be independent of temperature, the experimental scaling function data have been fitted to the empirical $F_{B F}(\Omega)$ function. In this fitting procedure parameters $A_{D_{1}}$ and $\tau_{D_{1}}$ of the high frequency Debye term and the 
asymptotic high frequency background parameter $B^{\prime}$ as well as the relaxation rate $\Gamma$ and the critical amplitude $S_{B F}$ have been fixed at values obtained by inter-and extrapolation of data from the broadband spectra and from light scattering measurements [97], respectively. Hence only $\tau_{D_{2}}$ and $A_{D_{2}}$ were adjusted to reach optimum agreement of scaling function data with the analytical form Eq.(4.26). Three runs and previous data were investigated [97]. The result of the regression analysis is shown by Fig.(5.37). Since the critical contribution resulted from subtraction of the noncritical parts from the total attenuation, the scatter in the $F(\Omega)$ data is rather large. Within the limits of this scatter, however, the data from different runs and different temperatures of measurement fall on one curve and agree with the empirical scaling function according to Bhattacharjee-Ferrell formalism, with $\Omega_{1 / 2}=2.1$. The finding of a longer relaxation time (14 ns $\leq \tau_{D_{2}} \leq 26 \mathrm{~ns}$ ) for aqueous solutions in [97] than pure non-associating TEA may reflect the collective redistribution of solvent molecules associated with the structural isomerization of the solute. This effect has been also reported in [129] mixtures water-alcohol. However, from the

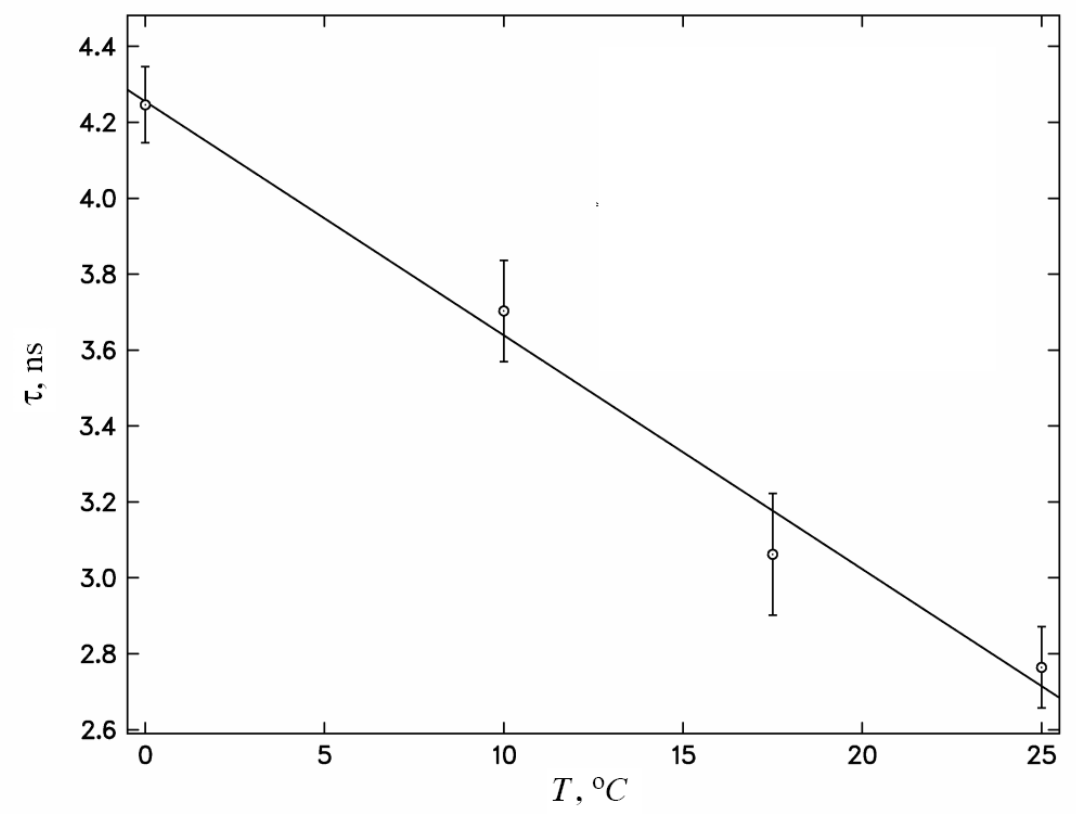

Figure 5.35: Relaxation time of structural isomerization of TEA versus temperature of the noncritical system triethylamine-2- propanol [135].

regression analysis results that both Debye relaxation times increase with $T$, at variance with Arrhenius or Eyring characteristics. An evaluation of the Eyring-Plot would produce a negative activation enthalpy. This behavior, which has been reported in [127] and [128] obviously is an indication of slowing down of chemical relaxations. This is an contro- 
versially discussed effect, which has been questioned by Milner and Martin [130] on the one side and confirmed by Procaccia [127], [27], Gitterman [132] as well as Krichevskii [134] and Wheeler [133] on the other side. Especially the low-frequency Debye term in frequency range of critical fluctuation contributions, assumed to be due to a uni-molecular reaction has to be considered with more details. According to the predictions by Proccacia et al. such uni-molecular reactions should be unaffected by the critical fluctuations. An example taken from literature [135] of a correct Arrhenius behavior of the non-aqueous and noncritical system triethylamine-2-propanol is presented in Fig.(5.35). Investigations

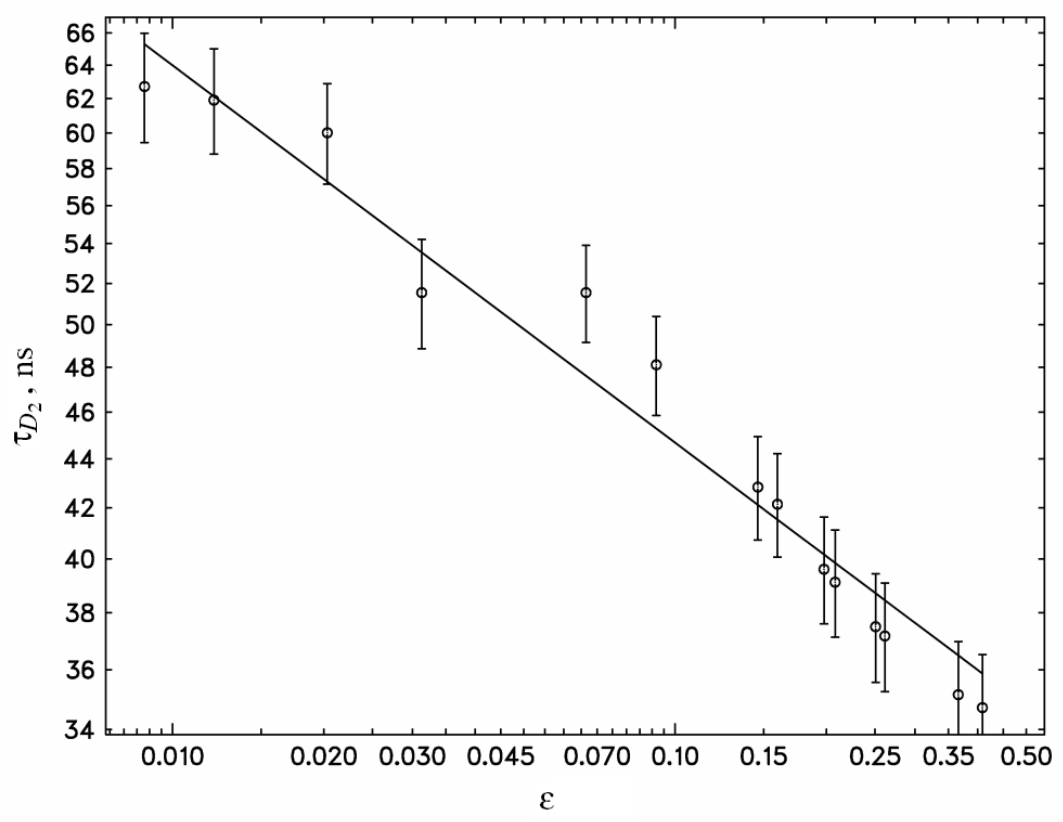

Figure 5.36: Bilogarithmic plot of the relaxation time $\tau_{D_{2}}$ of the (TEA-H $\left.-\mathrm{H}_{2} \mathrm{O}\right)$ system versus reduced temperature $\varepsilon$ : The line indicates the power law behavior, Eq.(5.23).

on that non-aqueous systems give once more an indication that collective redistribution of solvent molecules associated with structural isomerization of TEA in aqueous solution (TEA- $\mathrm{H}_{2} \mathrm{O}$ ) is the reason for the classic ${ }^{1}$ enlargement of relaxation times. Nevertheless, the (TEA- $\mathrm{H}_{2} \mathrm{O}$ ) critical ultrasonic data condense on a master curve if slowing down of this reaction is allowed Fig.(5.37). Moreover, according to the expression [128]:

$$
\tau_{D_{n}}=\tau_{D_{0, n}} \varepsilon^{-\psi}
$$

${ }^{1}$ classic, means not critical effects 
the temperature dependence of the low-frequency term ( $n=2$ in Eq.(5.23)), within the limits of experimental error, can be represent by a power law. Applying the relation defined by Eq.(5.23) yields $\tau_{D_{0,2}}=31.19 \mathrm{~ns}$ and $\psi=0.17 \pm 0.04$. This value of the critical exponent is in fair agreement with that from the system isobutyric acid-water [128], where Kaatze at al. obtained the exponents $\psi=0.2 \pm 0.05$ and $\psi=0.3 \pm 0.2$. In Fig.(5.36) a bilogarithmic plot of $\tau_{D_{2}}$ as a function of reduced temperature $\varepsilon$ is given. The unusual

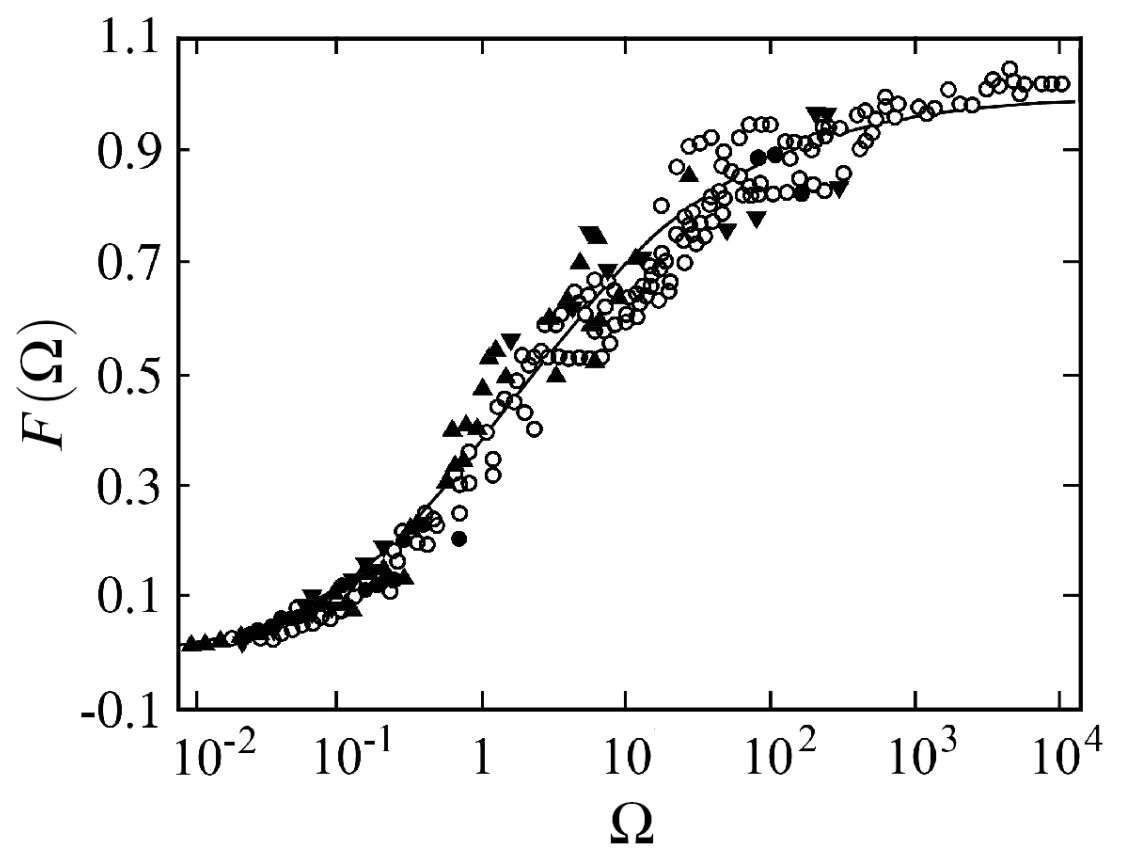

Figure 5.37: Scaling function data for the (TEA-H $\left.\mathrm{H}_{2} \mathrm{O}\right)$ system as calculated from runs special low frequency measurements and from previous broadband spectrometry [97] are indicated by figure symbols. (run $1(\bullet)$, run $2(\boldsymbol{\Delta})$, run $3(\boldsymbol{\nabla})$, previous $(0)$ ) The line is the graph of the empirical form of the Bhattacharjee-Ferrell scaling function.

coupling of the rotational isomerization to the critical fluctuations reflected thereby may be also be explained within the framework of the afore mentioned redistribution of solvent molecules associated with structural isomerization of TEA with water. More detailed, the ethylene groups parts of TEA are surrounded by cages of water molecules. The H-bond network of water molecules will fluctuated slower in these cages ("hydrophobic hydration"), which results in a higher viscosity. The Debye-Stokes-Einstein model, which predicts a linear relation between the rotational diffusion time of a solute molecule, $\tau_{\text {rot }}$ and viscosity $\eta_{s}$, may give an explanation for such effect:

$$
\tau_{r o t}=\frac{V \eta_{s}}{k_{B} T}
$$

where $V$ denotes the effective molecular volume. Unfortunately, the shear viscosity data 
in Fig.(5.32) do not exactly confirmed this hypothesis. The shear viscosity data increase close to the consolute point in the range of absolute temperature $T-T_{c}=0.5 \mathrm{~K}$, but the relaxation time data of the low-frequency term in the range of $7 \mathrm{~K}$. Furthermore, the viscosity of the (TEA- $\mathrm{H}_{2} \mathrm{O}$ ) mixture of critical composition varies near $T_{c}$ as $\eta_{s}=\eta_{0} \varepsilon^{-0.04}$ only. Hence, the power law behavior in the relaxation time of the $R_{D_{2}}^{+}(f)$ process cannot be solely due to effects of viscosity. There must exist an intrinsic mechanism that slows down near the critical demixing point. On the contrary, due to the exponent $v^{*}=0.664$ in the power law behavior of the mutual diffusion coefficient, it appears to be likely that the rotational isomerization is largely governed by the critical slowing of the mutual diffusion, $D(\varepsilon) \rightarrow 0$.

The non-Arrhenius behavior of the ultrasonic relaxation times associated with the protolysis (Eq.(5.19)) may also be taken to indicate slowing down of the chemical reactions near the critical point. Proccacia et al. have predicted effects of slowing of such reactions in which both constituents of a binary fluid participate. In Fig.(5.38) the relaxation time $\tau_{D_{1}}$ of reaction (5.19) is represented by Eq.(5.23) with $\tau_{D_{0,1}}=3.77$ and with the critical exponent $\psi=0.07 \pm 0.01$. In a binary mixture, there are two kinds of diffusion coeffi-

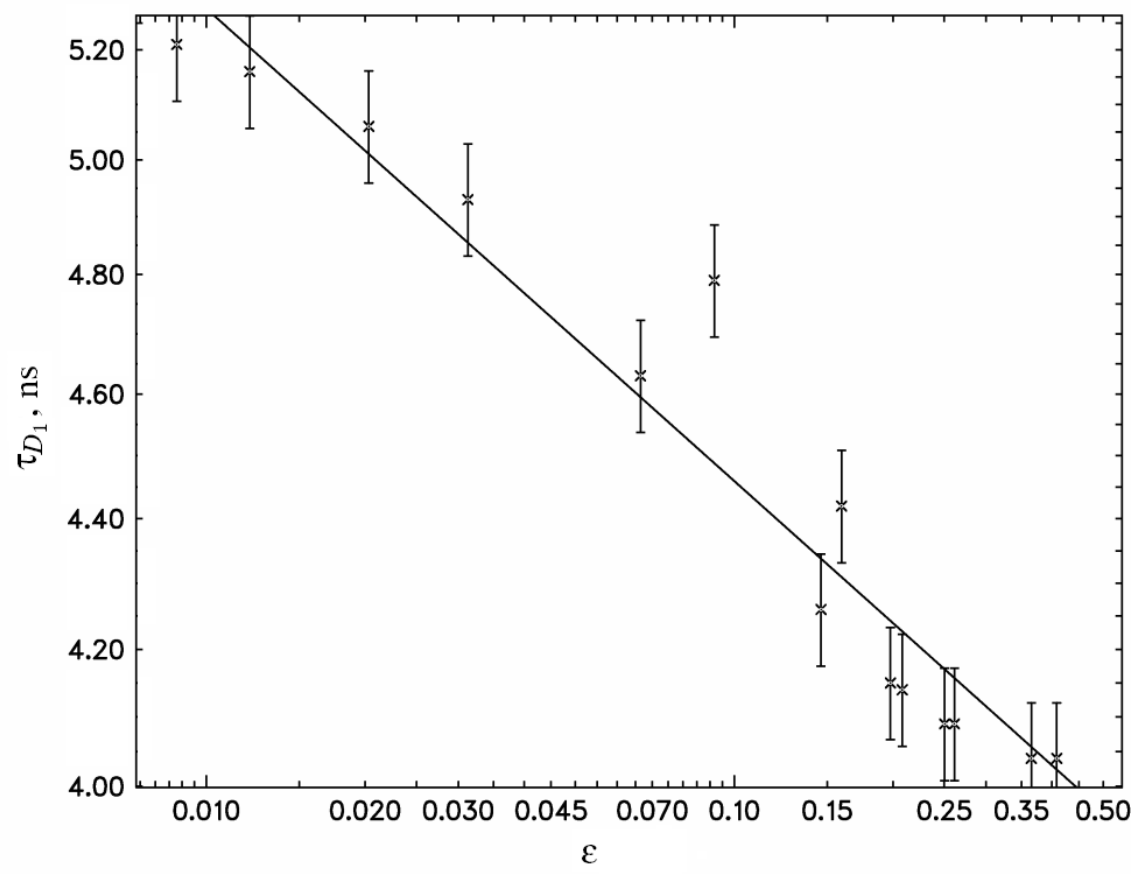

Figure 5.38: Bilogarithmic plot of the relaxation time $\tau_{D_{1}}$ of the (TEA-H $\left.\mathrm{H}_{2} \mathrm{O}\right)$ system versus reduced temperature $\varepsilon$ : The line indicates the power law behavior, Eq.(5.23). 


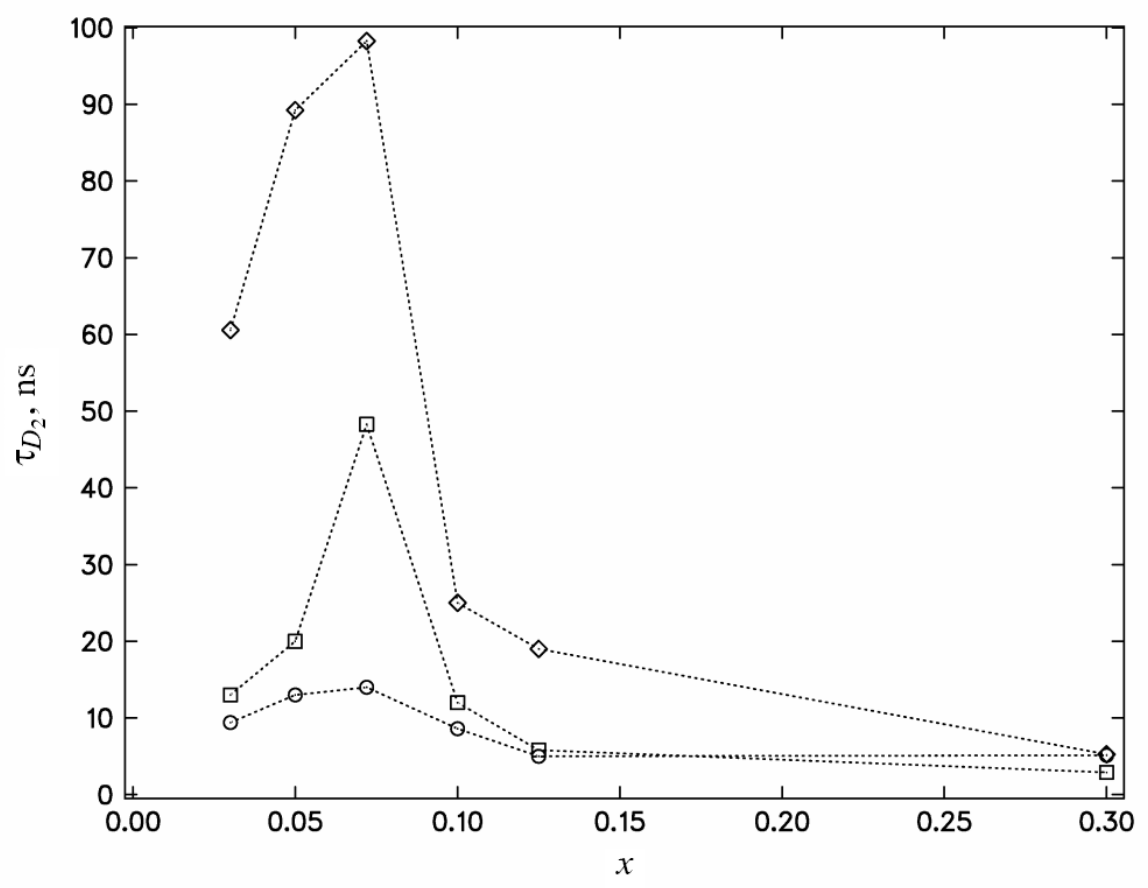

Figure 5.39: Relaxation time of the low frequency relaxation term of $\left(\mathrm{TEA}-\mathrm{H}_{2} \mathrm{O}\right)$ versus mole fraction of TEA. $\left((\circ) 10^{\circ} \mathrm{C},(\square) 15^{\circ} \mathrm{C},(\diamond) 17^{\circ} \mathrm{C}\right)$; Results from reevaluation of literature data [138].

cients: self-and mutual diffusion coefficients. The self-diffusion coefficient describes the mobility of individual molecule in the mixtures and it is defined for each component in a mixture. In [137], it was reported that the mutual diffusion coefficient is a collective property, which controls the mixing of the two components of a binary liquid. The protolysis reaction belongs to the types of reactions that are governed by the self diffusion coefficient. Smoluchowski has presented a relation between the forward (or backward) rate constant $k_{\text {diff }}$, controlled by the self diffusion coefficient $D_{A}$ of components $A$ and $D_{B}$ of component $B$ :

$$
k_{\text {diff }}=\text { const. } r_{A B}\left(D_{A}+D_{B}\right)
$$

where $r_{A B}=r_{A}+r_{B}$ denotes the minimal distance of interaction. The coupling between the self diffusion and mutual diffusion has been discussed in the literature [137] in the case of the system ethanol-water. According to that coupling, the mutual diffusion coefficient can be expressed as: 


$$
D=Q[\underbrace{\left(x_{A} D_{B}+x_{B} D_{A}\right)}_{L_{0}}-x_{A} x_{B}\left(\frac{f_{A A}}{x_{A}^{2}}+\frac{f_{B B}}{x_{B}^{2}}-2 \frac{f_{A B}}{x_{A} x_{B}}\right)],
$$

where $L_{0}$ denotes the self diffusion coefficient, with the mole fraction of components $A, B$, $x_{A}, x_{B}$ and the cross correlation functions and $f_{A B}$ and the auto correlation functions $f_{A A}$, $f_{B B}$. $Q$ is a thermodynamic factor. However, an analytical calculations of the self diffusion coefficients of the system (TEA- $\left.\mathrm{H}_{2} \mathrm{O}\right)$ are not possible, owning to the nonavailability of the velocity cross correlation functions of the solution.

In order to verify the presented results, additional literature data [138] of concentrationdependent broad band ultrasonic have been reevaluated. The results of the low frequency term, relaxation time $\tau_{D_{2}}$ versus mole fraction of TEA, are displayed in Fig.(5.39). The values are somewhat lager than previous data. Unfortunately, no information on the purity of TEA used in [138] is given. Furthermore, the critical temperature with $\left(T_{c}=17.6^{\circ} \mathrm{C}\right)$ is somewhat smaller than then one of this thesis $\left(T_{c}=18.21{ }^{\circ} \mathrm{C}\right)$. Nevertheless, qualitatively this result shows that critical slowing down of chemical relaxation occurs also in that process.

\subsection{Ternary system nitroethane-3-methylpentane-cyclohexane}

The investigations on binary systems without complex contributions presented in Section (5.3), have motivated to study of ternary mixtures within the framework of BhattacharjeeFerrell theory as well as the crossover formalism. Especially, due to the availability of experimental data for the binary systems nitroethane-cyclohexane (NE-CH) and nitroethane3-methylpentane (NE-3MP), it is interesting to examine the nature of a critical ternary mixture nitroethane-3-methylpentane-cyclohexane (NE-3MP-CH). The investigated ternary mixture belongs to the phase diagrams of type $2 \mathrm{a}$ (Sec.(2.4.2)). The aim of this study, in addition to the verification of the dynamic scaling hypothesis, is to compare the behavior of critical values in dependence of the concentration of the additional third component. Therefore, a careful investigation of the ternary phase diagram was performed, to verify the location of the plait line as well as of the col point. In present thesis the solvent (3MP) plays the role of the additional third component. The significant plait points of investigations are shown in Fig.(5.40) and the corresponding values are listed in Table (5.6) or in Table (5.5), respectively. The procedure of determination of the plait point line is based on the same procedure as for binary mixtures, namely the equal volume criterion. The studies in this thesis focused on the binary mixtures (NE-CH), (NE-3MP) as presented in Section (5.3), as well as on the ternary mixtures with the composition of non-col points $\alpha^{\prime}, \alpha^{\prime \prime}$, 


\begin{tabular}{lcccc}
\hline \hline mixtures & 3-methylpentane & $\begin{array}{c}\text { cyclohexane } \\
\text { compositions (weight fraction) }\end{array}$ & nitroethane & $T_{c}{ }^{\circ} \mathrm{C}$ \\
\hline (NE-CH) & 0.000 & 0.576 & 0.424 & 23.66 \\
n.col(NE-3M-CYC) $\alpha^{\prime}$ & 0.082 & 0.488 & 0.430 & 22.43 \\
col (NE-3M-CYC) & 0.200 & 0.360 & 0.440 & 21.33 \\
n.col (NE-3M-CYC) $\alpha^{\prime \prime}$ & 0.300 & 0.260 & 0.440 & 22.45 \\
(NE-3MP) & 0.534 & 0.000 & 0.466 & 26.44 \\
\hline \hline
\end{tabular}

Table 5.5: Critical parameters of the mixtures investigated.

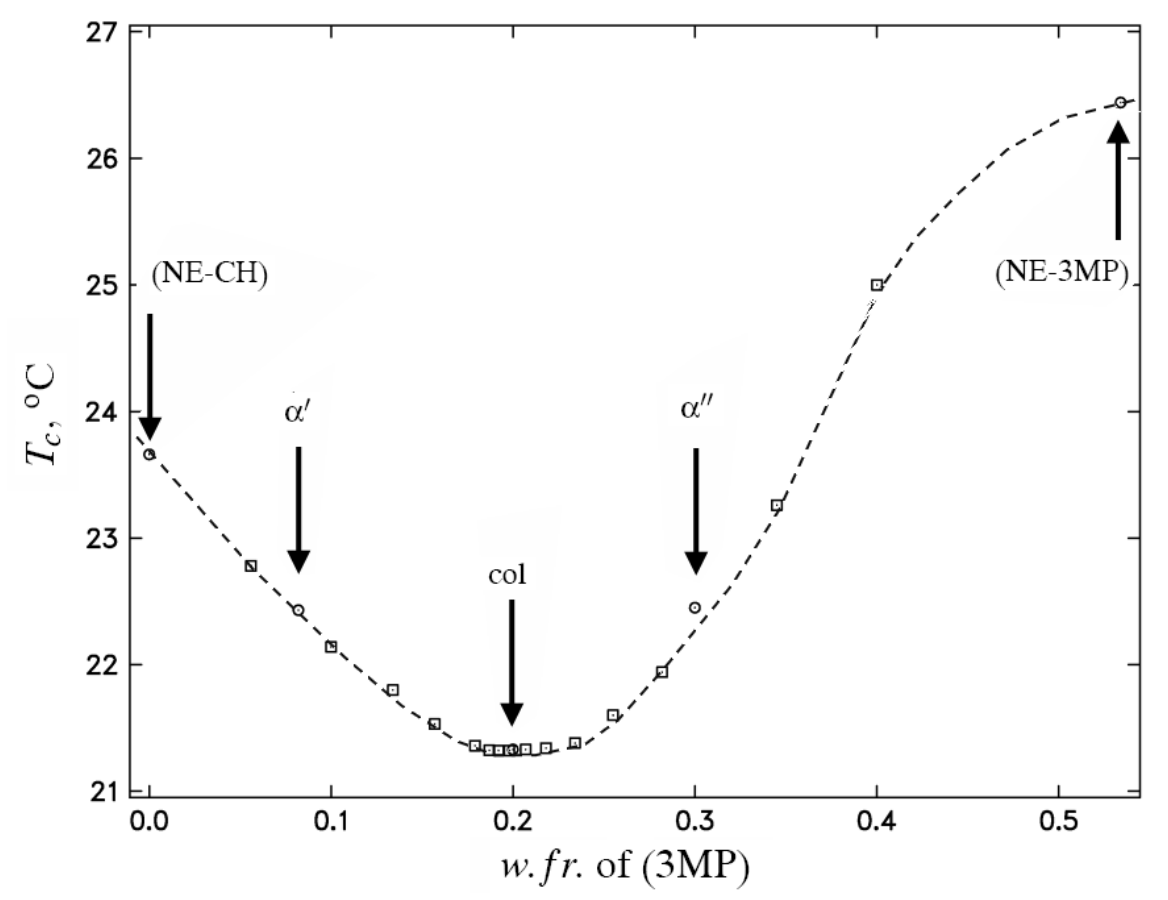

Figure 5.40: The plait point line as a function of critical (3MP) content, from the binary system (NE-CH) to (NE-3MP): $\circ$ denote plait points, including the col point (see Tables (5.5) and (5.6)).

and with the col(or saddle)-point composition. The critical compositions and critical temperatures are presented in Table (5.5). The ternary mixtures had a constant weight fraction relationship of $(\mathrm{CH})$ and $(\mathrm{NE}),\left(w \cdot f r \cdot C H / w \cdot f r_{\cdot N E}=\right.$ const.). In Fig.(5.41) three plots of density versus reduced temperature are presented. However, as was mentioned before in Section (4.5), although the crossover theory has been developed for binary mixtures, the ternary system (NE-3MP-CH) belongs to the same universality class for dynamical properties as the investigated binary fluids. Nevertheless, within the scope of investigation on 


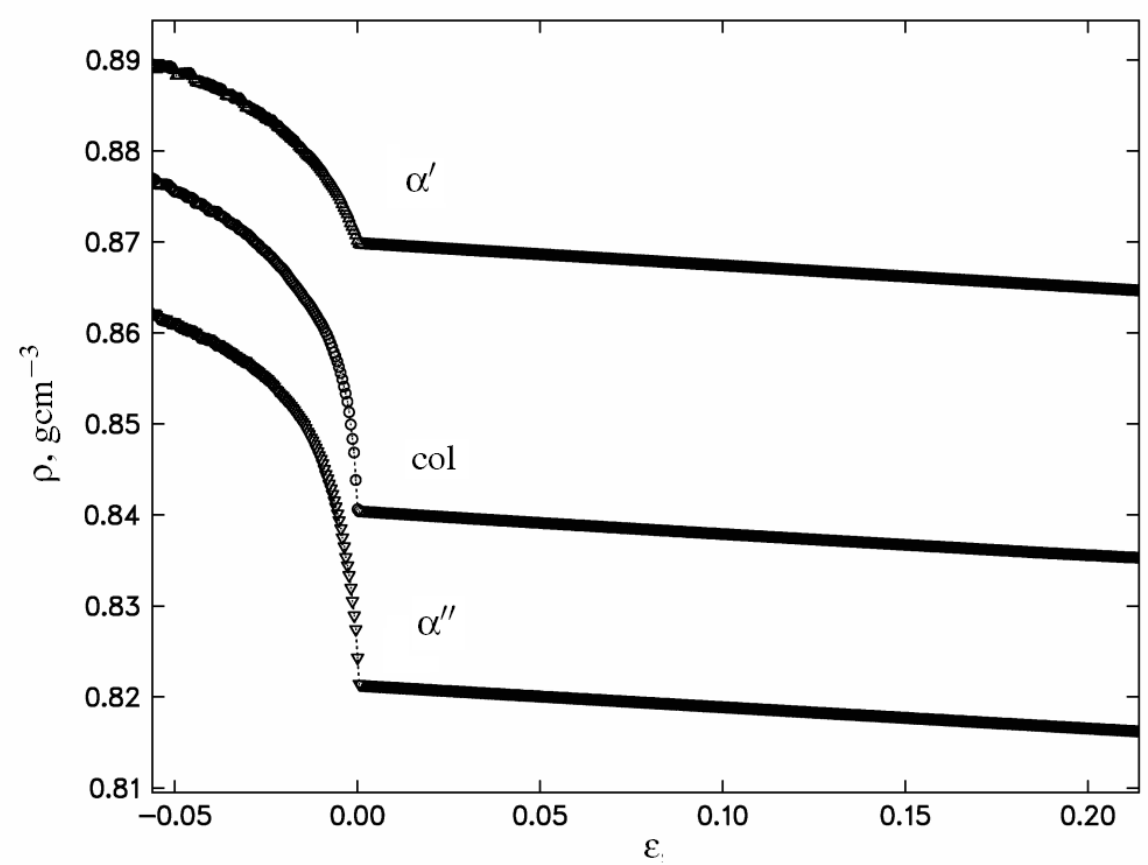

Figure 5.41: Density plots of ternary mixtures: $\Delta$, denotes the $\alpha^{\prime}$ mixture, o the col mixture and $\nabla$ the $\alpha^{\prime \prime}$ mixture.

\begin{tabular}{cc|cc}
\hline \hline W.fr. of 3-MP & $T_{c}{ }^{\circ} \mathrm{C}$ & W.fr. of 3-MP & $T_{c}{ }^{\circ} \mathrm{C}$ \\
\hline 0.000 & $23.66^{(N E-C H)}$ & 0.200 & $21.33^{\text {col }}$ \\
0.056 & 22.78 & 0.207 & 21.33 \\
0.082 & $22.43^{\text {n.col } \alpha^{\prime}}$ & 0.218 & 21.34 \\
0.100 & 22.14 & 0.234 & 21.38 \\
0.134 & 21.80 & 0.255 & 21.60 \\
0.157 & 21.53 & 0.282 & 21.94 \\
0.179 & 21.36 & 0.300 & $22.45^{\text {n.cold }}{ }^{\prime \prime}$ \\
0.187 & 21.32 & 0.345 & 23.26 \\
0.192 & 21.32 & 0.400 & 25.00 \\
0.198 & 21.32 & 0.490 & $26.44^{(3 M P-C H)}$ \\
\hline \hline
\end{tabular}

Table 5.6: Parameters of critical points in dependence of weight fraction $(w f)$ of (3MP) from Fig.(5.40).

ternary mixture, much attention has been directed on the behavior of the crossover effects in the data evaluation of ternary mixtures. Especially, the dependence of cut-off wave 
numbers $q_{c}$ and $q_{D}$ on the background viscosity $\eta_{b g}$ has been studied with more details as in the case of binary fluids. The measurements of viscosity, light scattering as well as ultrasonic attenuation have been performed with the same instrumental setup as for the other critical mixtures with the critical parameters of the mixtures in Table (5.5).

\subsubsection{Crossover studies of viscosity and light scattering}

In order to determine the dependence of the cut-off wave numbers $q_{c}$ and $q_{D}$ from the background viscosity $\eta_{b g}$, parameters $A_{\eta}, B_{\eta}$ and $T_{\eta}$ the shear viscosity measurement have been evaluated for each ternary mixture. In Table (5.7) the parameters from a simultaneous treatment of $\eta_{s}$ and $D$ data, taking crossover as well as background effects in account, according to Eqs.(4.29), are presented. The plots of parameter $A_{\eta}, B_{\eta}$ and $T_{\eta}$

\begin{tabular}{|c|c|c|c|c|c|c|c|}
\hline $\begin{array}{c}\text { weight fraction } \\
\text { of (3MP) }\end{array}$ & $\begin{array}{r}A_{\eta} \pm 0.02 \\
\cdot 10^{-6} \mathrm{Pas} \\
\end{array}$ & $\begin{array}{c}B_{\eta} \pm 200 \\
\mathrm{~K}\end{array}$ & $\begin{array}{c}T_{\eta} \pm 20 \\
\mathrm{~K}\end{array}$ & $\begin{array}{c}\xi_{0} \pm 0.02 \\
\mathrm{~nm}\end{array}$ & $\begin{array}{c}q_{c} \\
\mathrm{~nm}^{-1}\end{array}$ & $\begin{array}{c}q_{D} \pm 0.06 \\
\mathrm{~nm}^{-1}\end{array}$ & $\begin{array}{l}\Gamma_{0} \pm 2.5 \\
\cdot 10^{9} \mathrm{~s}^{-1} \\
\end{array}$ \\
\hline 0.000 & 0.55 & 2537 & -51 & 0.160 & $>1000$ & 0.55 & 156 \\
\hline 0.082 & 0.59 & 2958 & -134 & 0.195 & $4.40(10)$ & 0.80 & 124 \\
\hline 0.200 & 0.61 & 3174 & -174 & 0.213 & $14.40(77)$ & 0.60 & 102 \\
\hline 0.300 & 0.50 & 2818 & -111 & 0.209 & $12.89(68)$ & 0.52 & 117 \\
\hline 0.534 & 0.23 & 2877 & -84 & 0.230 & $1.30(12)$ & 0.32 & 125 \\
\hline
\end{tabular}

Table 5.7: Parameters from shear viscosity and light scattering measurements in dependence on the weight fraction of (3MP): the background viscosity was determined according to Eqs.(4.29).

versus the weight fraction of (3-MP) are presented in Fig.(5.42) and Fig.(5.43) according to Table (5.7). It is interesting to see that quantity $A_{\eta}$ follows the inverse shape of the in Fig.(5.40) shown plait point temperature in dependence on (3MP) concentration. On the one hand similar situation can be observed in case of the parameter $B_{\eta}$ in the limits of error. On the other hand, the quantity $T_{\eta}$ relates to the plait point plot in Fig.(5.40). The central parameter the fluctuation correlation length $\xi_{0}$ within the framework of analysis of shear viscosity and light scattering measurements is presented in Fig.(5.44). Within the limit of errors, it demonstrates an increase, controlled by the weight fraction of (3MP). Along with the graph of Eq.(5.9) the dependence of the correlation length amplitude upon the scaled temperature is shown as bilogarithmic plot in Fig.(5.44) for each ternary as well as binary mixture (Table (5.7)). The fluctuation correlation length plots follow power law over significant range of reduced temperature. The amplitude $\xi_{0}$, of the (NE-3MP) system is significantly larger than that of the (NE-CH) system Table (5.7). This difference in the fluctuation correlation length of similar binary mixtures is obviously a reflection of their different shear viscosities, Fig.(5.45). This assumption is supported by the behavior of the $\xi_{0}$ values of the ternary mixtures $\alpha^{\prime}$, col and $\alpha^{\prime \prime}$, which are following of order of weight fraction likewise the shear viscosity $\eta_{s}$. In Fig.(5.47), the characteristic relaxation 

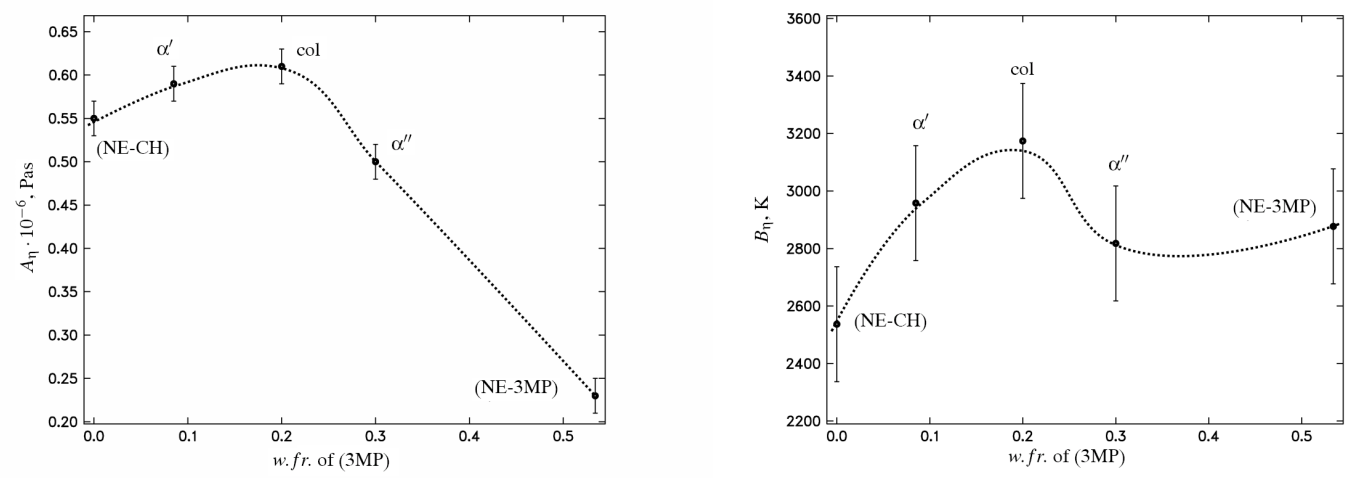

Figure 5.42: Plots of Table (5.7) resulted from the viscosity background determination with the aid of Eqs.(4.29) and the crossover formalism versus weight fraction of (3MP), lines are drawn to guide the eyes; Left Figure: plot of parameter $A_{\eta}$; Right figure: plot of parameter $B_{\eta}$.
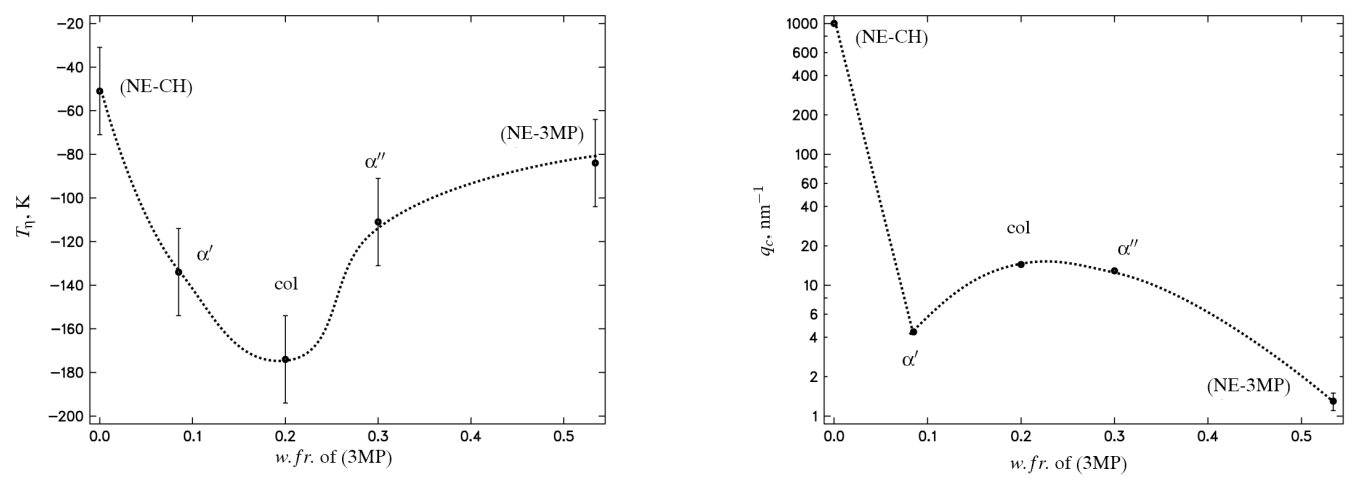

Figure 5.43: Plots of Table (5.7) resulted from the viscosity background determination with the aid of Eqs.(4.29) and the crossover formalism versus weight fraction of (3MP), lines are drawn to guide the eyes; Left Figure: plot of parameter $T_{\eta}$; Right figure: semilogarithmic plot of the cut-off wave number $q_{c}$.

rate amplitudes $\Gamma_{0}$ are displayed. The presented data correspond once more with the plait point line in Fig.(5.40). Finally, the conclusion could be done, that in ternary mixtures of type $2 \mathrm{a}$, the col point corresponds with the smallest value of the characteristic relaxation rate $\Gamma_{0}$ between two corresponding binary mixtures. Figure (5.48) shows three relaxation rates $\Gamma(\varepsilon)$, of order of fluctuations which according to Eq.(4.11) have been calculated from the mutual diffusion coefficient Eq.(4.35) and the fluctuation correlation length of the critical ternary systems $\alpha^{\prime}$, col and $\alpha^{\prime \prime}$. The diffusion coefficient data exhibit systematic deviations from function Eq.(4.35). These deviations may result from the insufficient temperature control $( \pm 0.03 \mathrm{~K})$. Especially in the case of the ternary mixture $\alpha^{\prime \prime}$ the relaxation rate data exhibit small deviations from the power-law behavior. However, the cut-off 

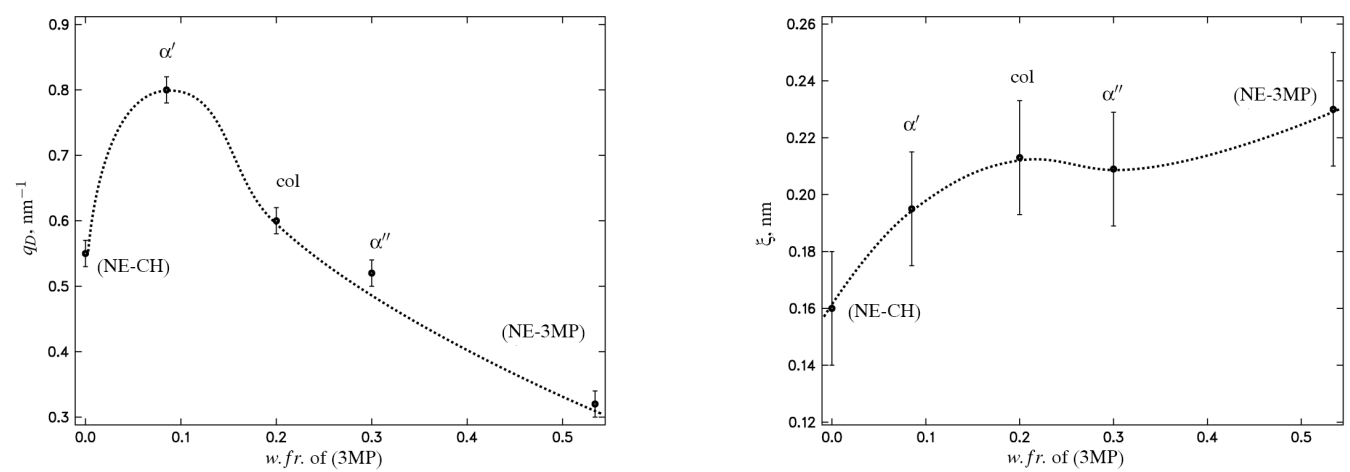

Figure 5.44: Plots of Table (5.7) resulted from the viscosity background determination, according to Eqs.(4.29), lines are drawn to guide the eyes; Left Figure: plot of the cut-off wave number $q_{D}$; Right figure: plot of the correlation length amplitude $\xi_{0}$.

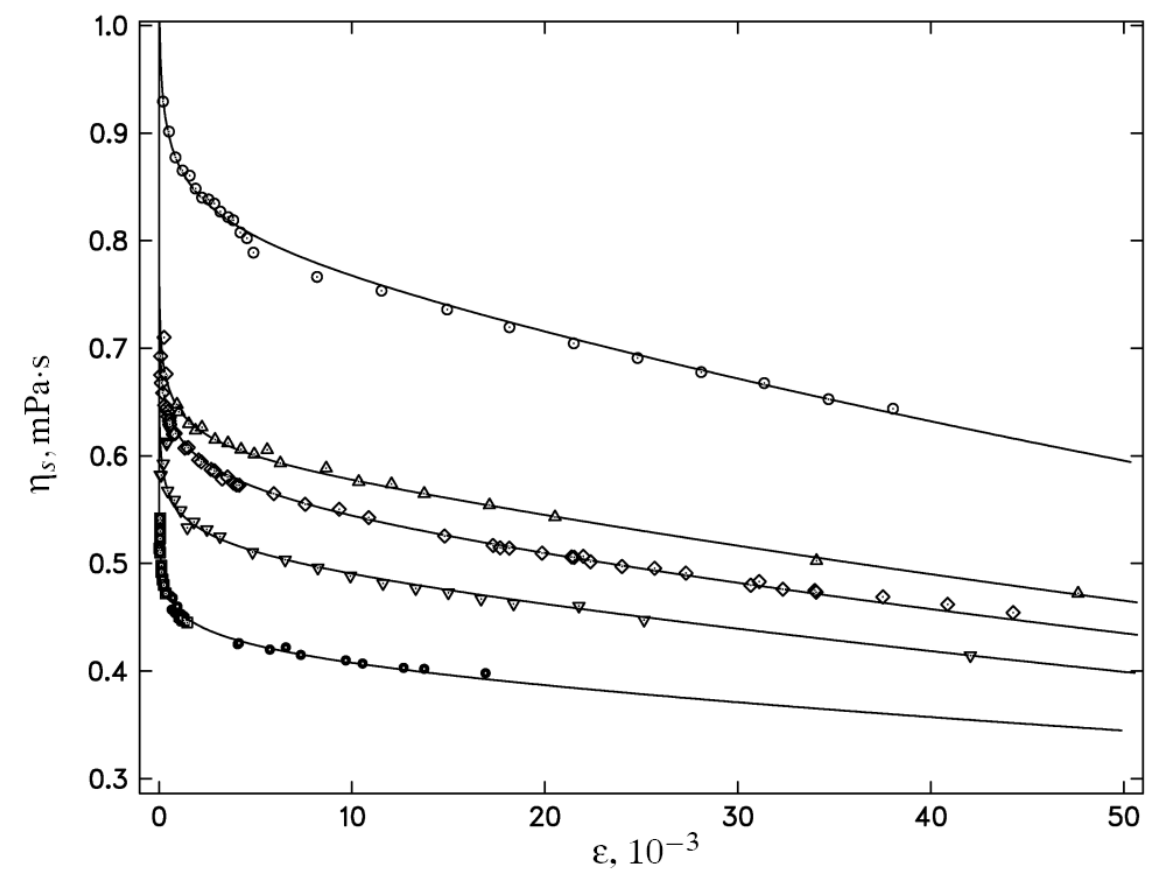

Figure 5.45: Shear viscosity plots versus reduced temperature $\varepsilon$ : of (NE-CH), $\circ ; \alpha^{\prime}, \Delta$; $\mathrm{col}, \diamond ; \alpha^{\prime \prime}, \nabla ;(\mathrm{NE}-3 \mathrm{MP}), \bullet$.

wave numbers in Fig.(5.43) and Fig.(5.44) display an unexpected shape. Especially the $q_{c}$ parameter with value $>1000$ of the crossover function $H\left(\xi(\varepsilon), q_{c}, q_{D}\right)$ in the case of $(\mathrm{NE}-\mathrm{CH})$ does not fit to the qualitative shape of the other values. Nevertheless, taken 

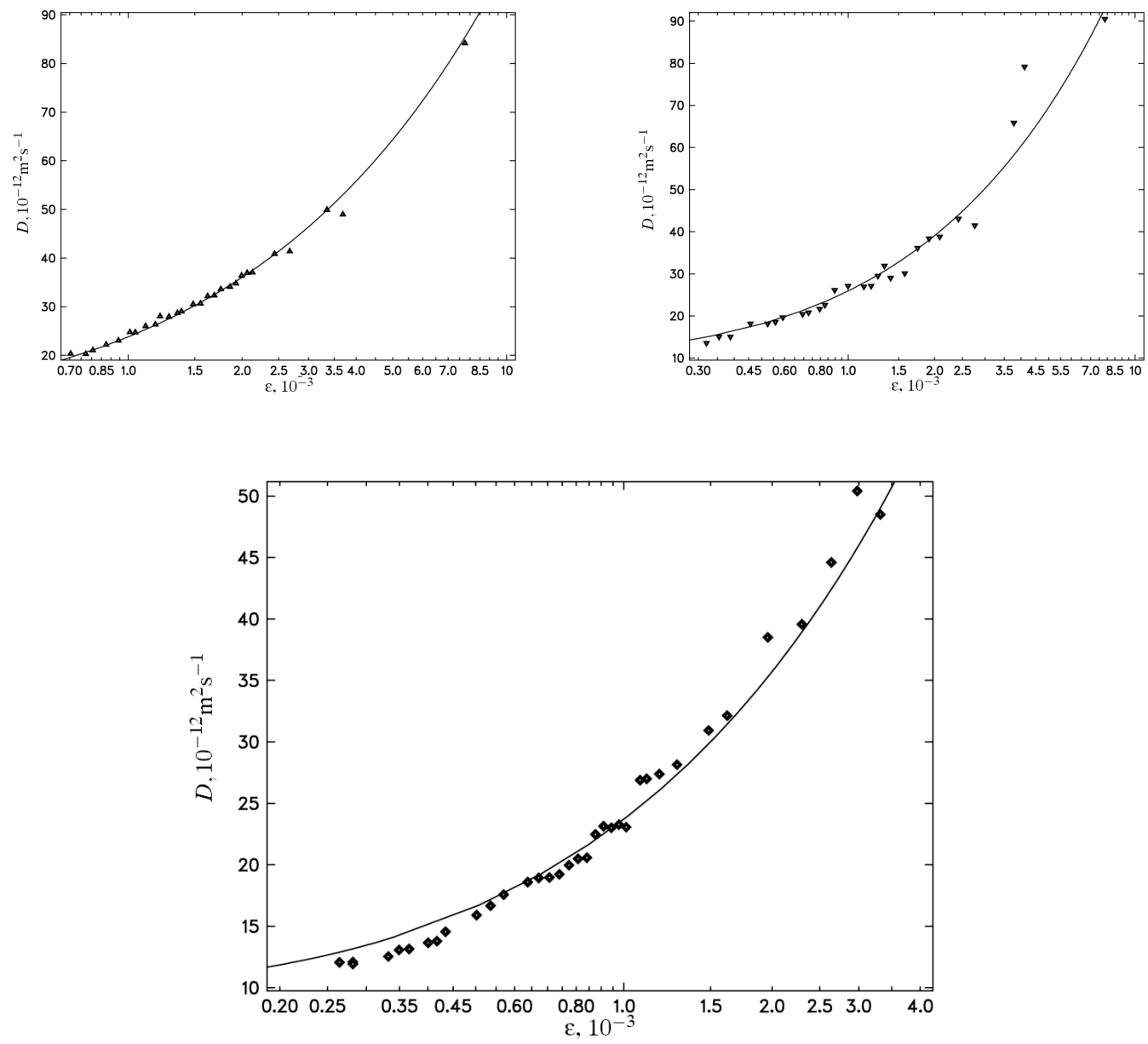

Figure 5.46: Semilogarithmic plots of mutual diffusion coefficient, according to Eq.(4.35);Left Figure: ternary mixture $\alpha^{\prime}$; Right figure: ternary mixture $\alpha^{\prime \prime}$; Lower figure: ternary mixture at col point.

into account that $q_{c}$ is one of the parameters which are controlling the overlap between mean field and critical range, the unusual behavior corresponds with the anomaly of critical amplitude (see Sec.(5.5.2)) $S_{B F}$ in (NE-CH). However, this correspondence is only an assumption. Another question which should be treated within framework of dynamic light scattering is the behavior of critical exponents. The assumption has been made that adding a third component to a binary critical mixture does not only alter the critical exponents but also change or influence the correction to scaling. In fact, in literature this kind of behavior has been reported [41] in the case of the ternary mixture aniline-cyclohexanep-xylene. In that work it was observed that, due to the existence of the third component, an enlargement of the critical static and dynamic exponents results. In the present thesis, 

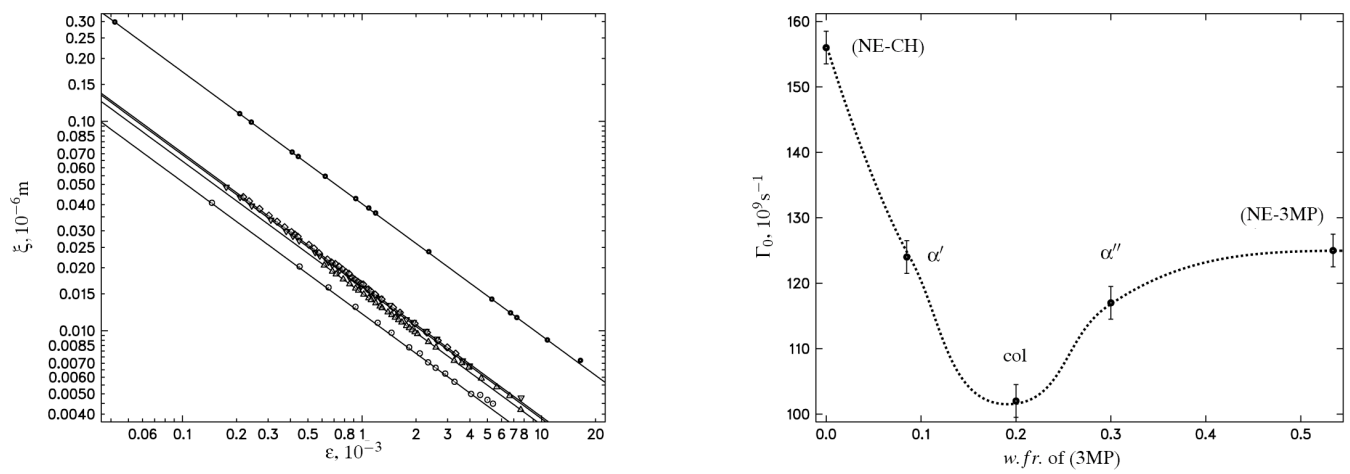

Figure 5.47: Plots of Table (5.7) resulted from the viscosity background determination, according to Eqs.(4.29), of investigated binary and ternary mixtures; Left Figure: bilogarithmic plot of the fluctuations correlation length $\xi_{0}$ versus reduced temperature $\varepsilon$, (NE-CH), ०; $\alpha^{\prime}$, $\triangle$; col, $\diamond ; \alpha^{\prime \prime}, \nabla ;(\mathrm{NE}-3 \mathrm{MP}), \bullet$; Right figure: the characteristic relaxation rate $\Gamma_{0}$ versus weight fraction of (3MP), lines are drawn to guide the eyes.

as mentioned before, owing to insufficient temperature control $( \pm 0.03 \mathrm{~K})$ in comparison with $( \pm 0.001 \mathrm{~K})$ in [41], the determination of critical exponents was not possible. Hence, critical exponents from theory, listed in Chapter 2, have been used. However, the results in this section, support the critical exponents for binary mixtures.

\subsubsection{Dynamic scaling function of a ternary mixture}

Conflicting results have been reported for ternary systems with critical demixing point with regard to decreasing of the magnitude of critical sound absorption as the number of components is increased, [140]. On the one side, one of Mistura's conclusions was that in fact the experimental critical absorption decrease along the plait line between corresponding binary systems, on the other side D'Arrigo's et al. reported a opposite case in the ternary system methanol-nitrobenzene-iso-octane [139]. At frequencies $f$ between $200 \mathrm{kHz}$ and $400 \mathrm{MHz}$ the ultrasonic attenuation coefficient $\alpha$ of the mixture of the ternary mixtures $\alpha^{\prime}$, col as well as $\alpha^{\prime \prime}$ has been measured using two different methods and four specimen cells.

In the frequency range from $200 \mathrm{kHz}$ to $400 \mathrm{MHz}$ the ultrasonic spectra of the two temperatures of the ternary system with the col point composition are displayed in frequency normalized format in Fig.(5.49). At high frequency the $\left(\alpha / f^{2}\right)$ data tend towards a frequency independent value, representing the background contribution. However, the broad band ultrasonic spectrum cannot be represented only by the critical contribution according to Bhattacharjee-Ferrell theory and a frequency independent $B^{\prime}$. The error in the data from the regression analysis, exhibits some systematic deviation. Therefore, additional 

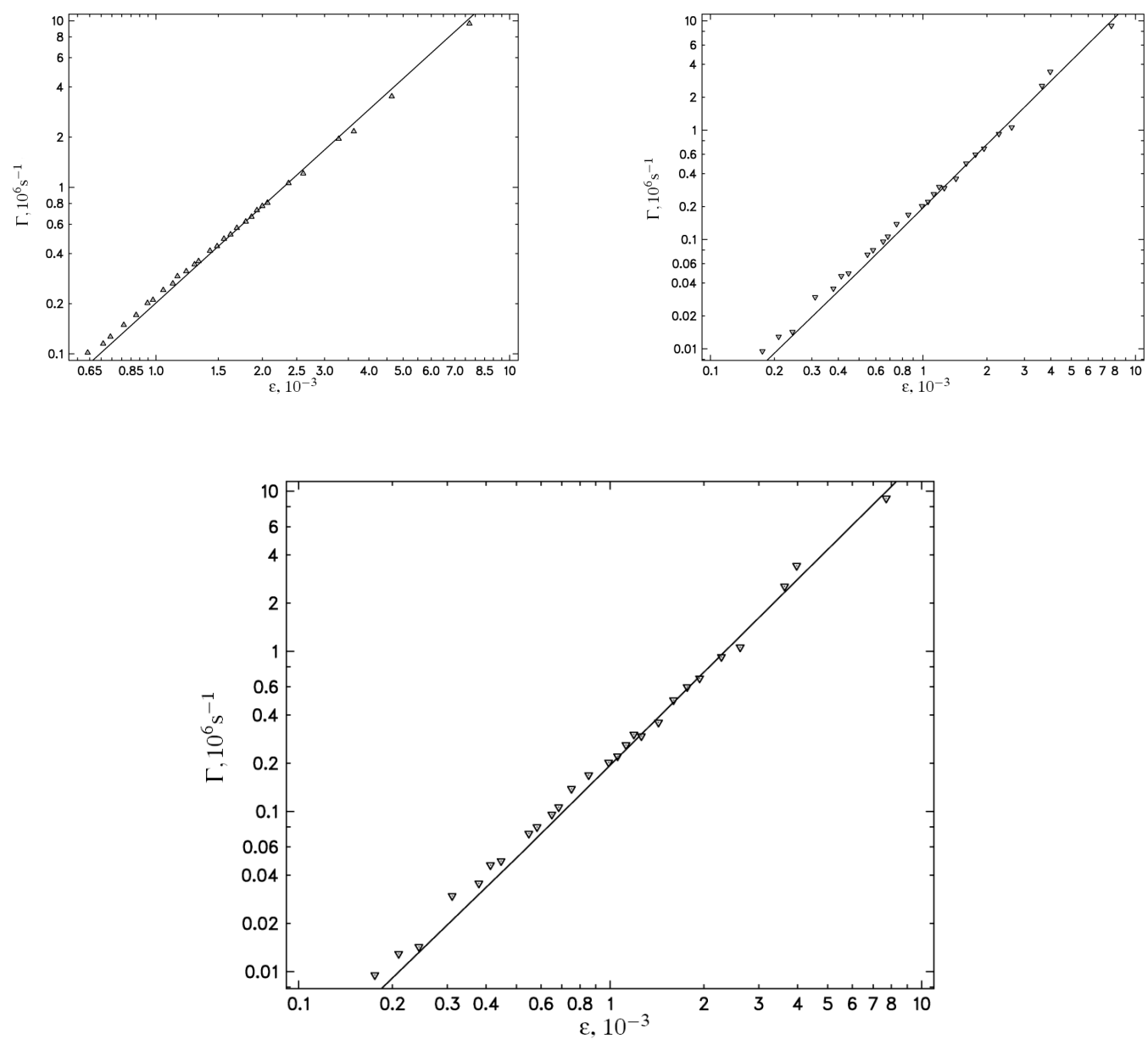

Figure 5.48: Plots of relaxation rate of fluctuations $\Gamma(\varepsilon)$; Left Figure: ternary mixture $\alpha^{\prime}$; Right figure: ternary mixture $\alpha^{\prime \prime}$; Lower figure: ternary mixture at col point.

ultrasonic measurement of non critical composition of mixtures (NE-CH) and (NE-3MP), with $2.5 \%$ of (NE) at the three temperatures $20^{\circ} \mathrm{C}, 25^{\circ} \mathrm{C}$ and $30^{\circ} \mathrm{C}$ have been performed, in order to identify an additional relaxation process. Debye relaxation process $R_{D}^{+}(f)$ in the nanosecond range has been found. Unfortunately, concerning this Debye relaxation term, no information could be found in the literature. An assumption is, that the relaxation term reflects an association process of (NE) similar to that of methanol in hexane, [93]. The investigated ultrasonic attenuation spectra of the non-col point mixtures $\alpha^{\prime}$ and $\alpha^{\prime \prime}$ display the same characteristic, Fig.(5.50). In order to determine the lower part of ultrasonic spectra, where the critical contributions predominates at temperatures between $30{ }^{\circ} \mathrm{C}$ and the $T_{c}$ of $\alpha^{\prime}$, col, $\alpha^{\prime \prime}$, two runs for every mixture have been performed in frequency range between $100 \mathrm{kHz}$ and $7 \mathrm{MHz}$. In Fig.(5.51), the excess attenuation spectra 


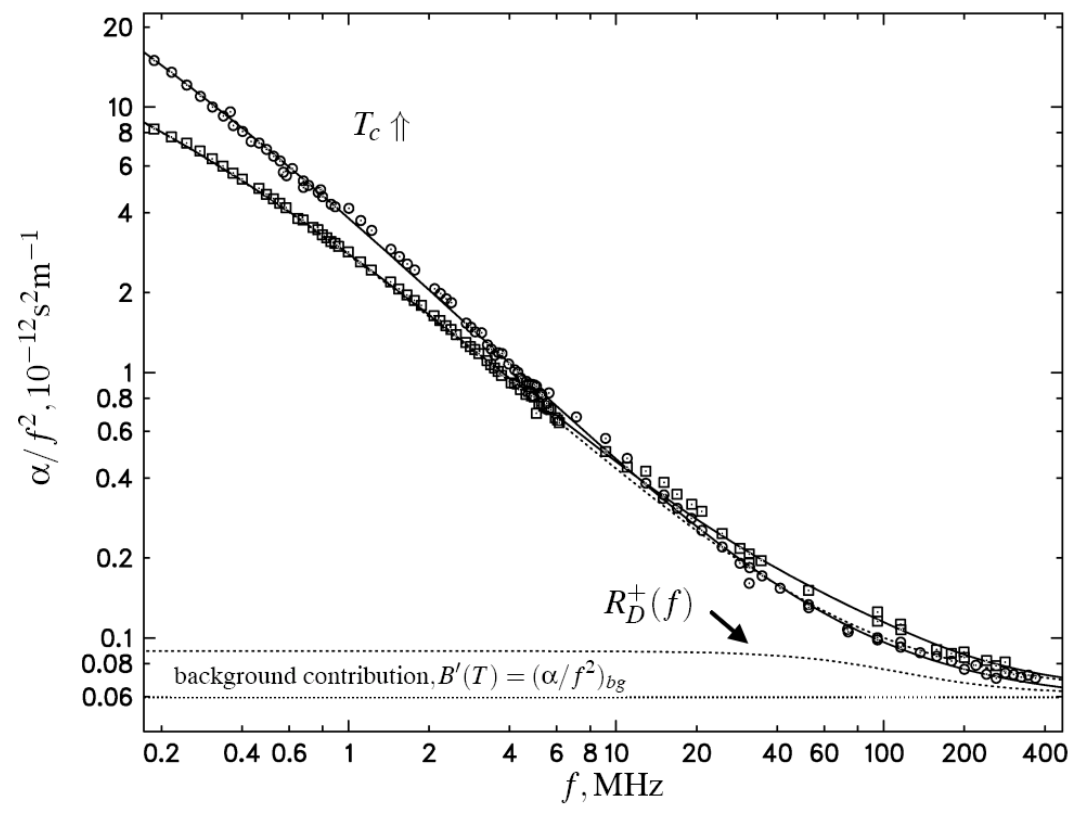

Figure 5.49: Frequency normalized ultrasonic attenuation spectra of the ternary mixture of col point composition: $\circ$ correspond to data close to consolute point $\simeq T_{c}$, and $\square$ represent ultrasonic data at temperature $23{ }^{\circ} \mathrm{C}$.

of the binary as well as ternary mixtures of critical composition are displayed close to the consolute point. With respect to the main results of Mistura and D'Arrigo [139], [140], another conclusions has to be drawn in the case of the ternary mixture (NE-3MP-CH). The sound attenuation does not change in dependence on the number of components. The low frequency ultrasonic data displayed in Fig.(5.51) rather follow the behavior of the fluctuation correlation length $\xi_{0}$ amplitude (small right plot in Fig.(5.51)). According to the Bhattacharjee-Ferrell dynamic scaling model the low frequency ultrasonic measurements, with the relaxation rate from dynamic light scattering have been evaluated for the $\alpha^{\prime}$, the col point and the $\alpha^{\prime \prime}$ ternary mixtures, with the critical amplitude $S_{B F}$ as the only adjustable parameter, according to Eq.(4.26), with respect to the Debye relaxation term. The data nicely fit to the empirical scaling function $F_{B F}$, Fig.(5.52). The scaling function data in the case of the ternary mixture $\alpha^{\prime \prime}$ exhibits a remarkable scatter, due measurement problems, caused by rather high sound attenuation. Within the limits of this scatter, however, the data from different runs and different temperatures of measurement fall on one curve and agree with the empirical scaling function. Unfortunately, the critical amplitude in all three mixtures demonstrates a dependence on temperature, with smaller magnitude but similar to that of the binary system (NE-CH). However, the additional relaxation con- 


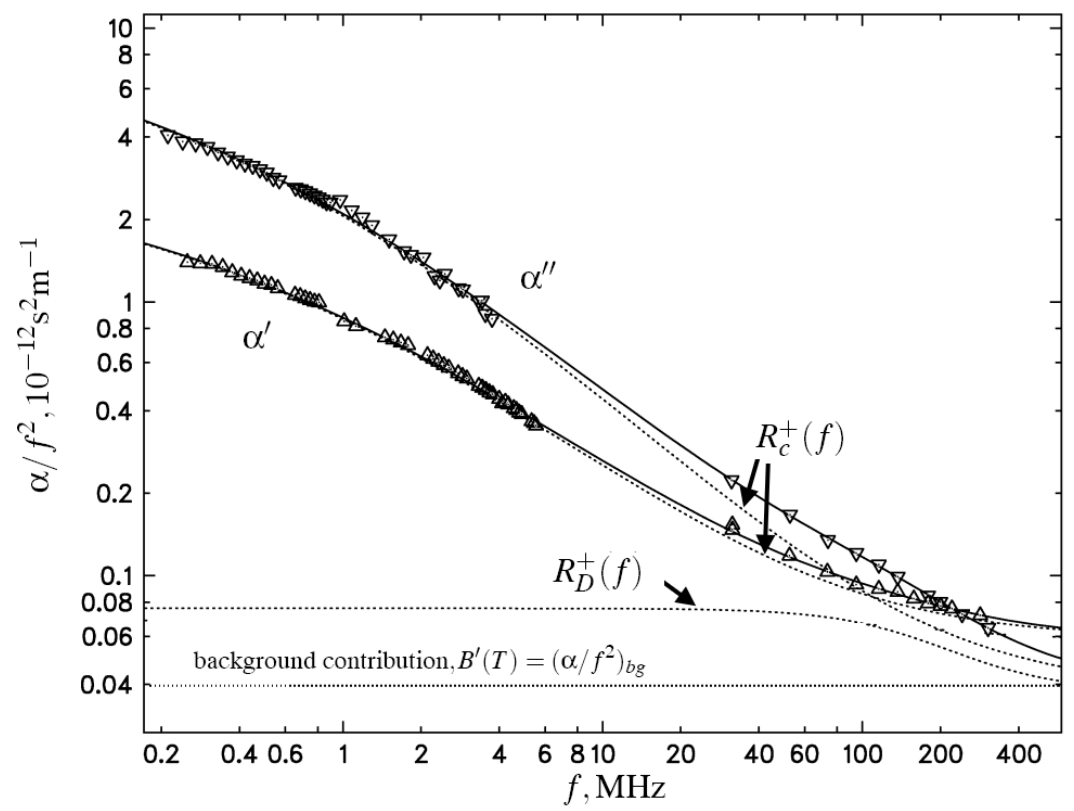

Figure 5.50: Frequency normalized ultrasonic attenuation spectra of the ternary $\alpha^{\prime}, \Delta$, and $\alpha^{\prime \prime}, \nabla$ mixtures at $25^{\circ} \mathrm{C}$.
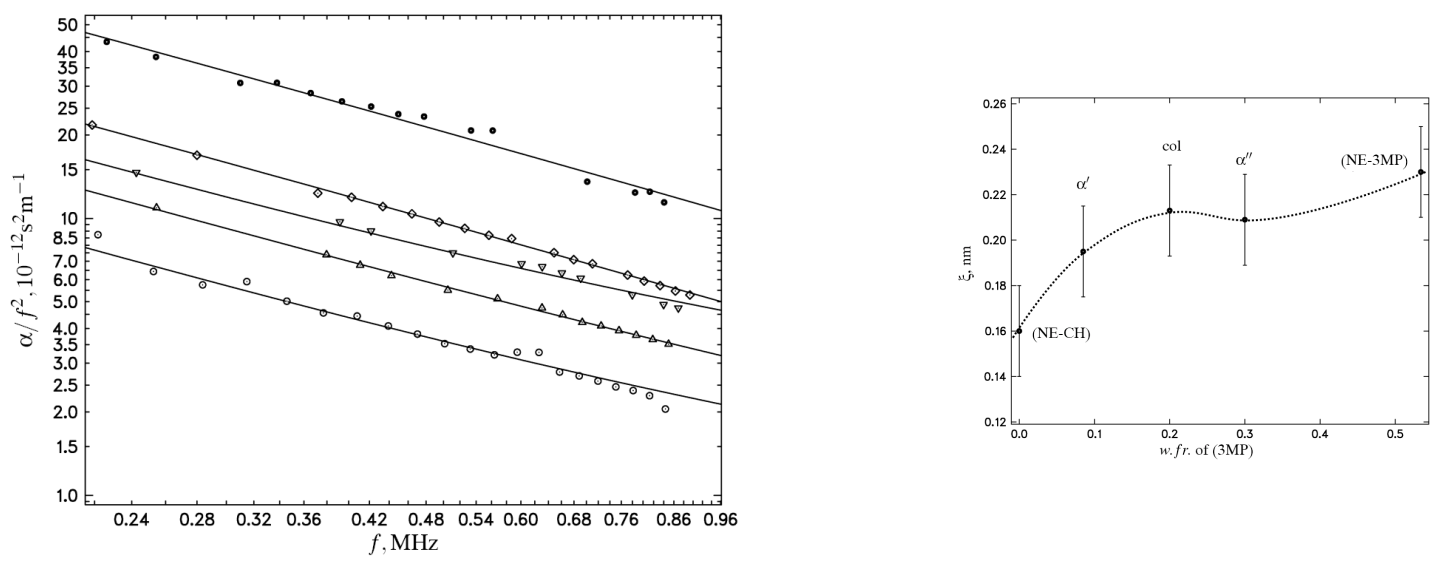

Figure 5.51: Left Figure: plots of low frequency part of the frequency normalized ultrasonic spectra of the binary mixtures (NE-CH), $\circ$ and (NE-3MP), $\bullet$ as well as of the ternary mixtures $\alpha, \Delta$, col point $\diamond$ and $\alpha^{\prime \prime}, \nabla$ at critical temperature $T_{c}$; Right figure: re-plot of Fig.(5.44) of the fluctuation correlation function $\xi_{0}$ versus the concentration in weight fraction of (3MP). 

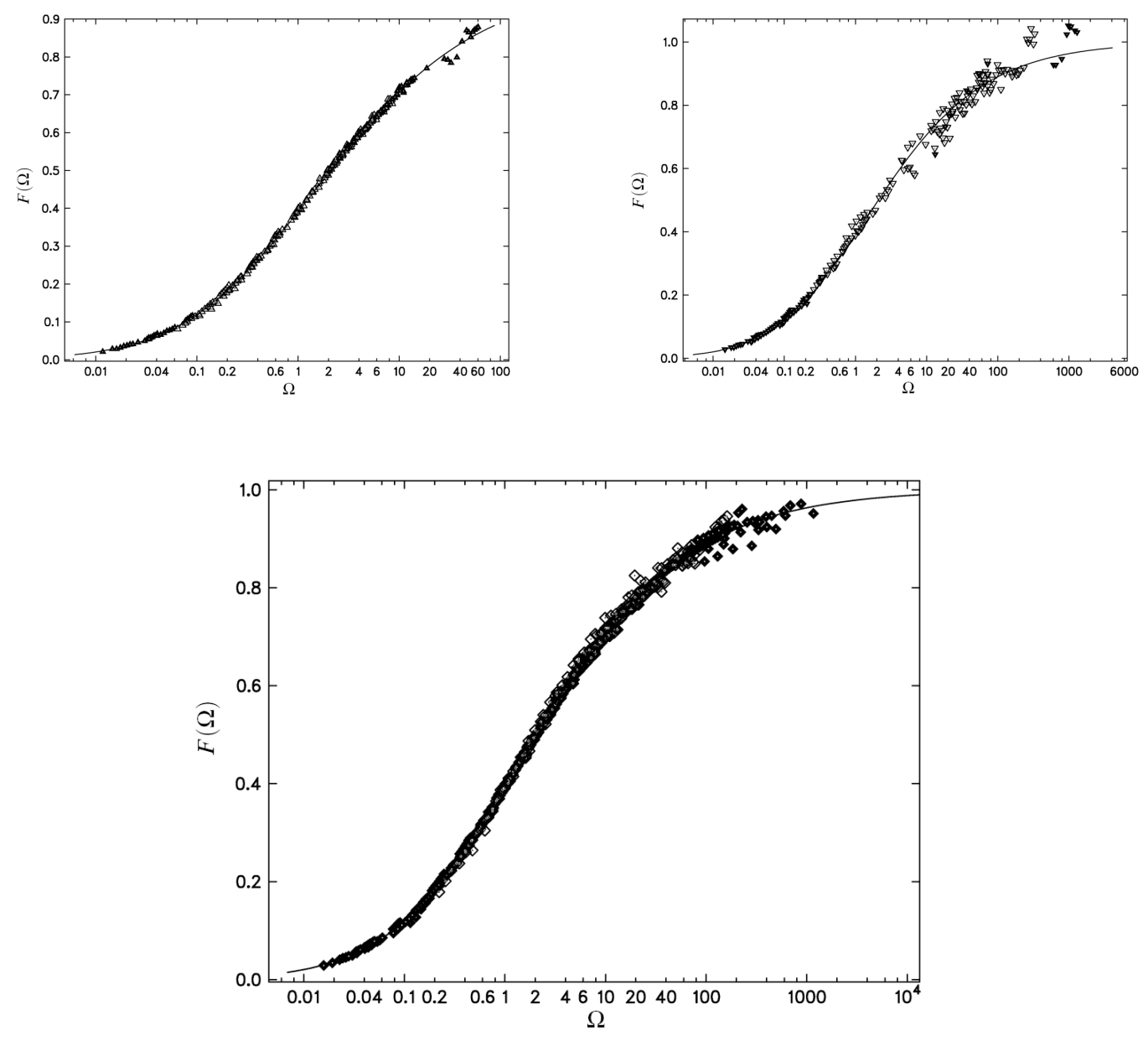

Figure 5.52: Scaling function plots according to the Bhattacharjee-Ferrell theory, with $\Omega_{1 / 2}=2.1$; Left Figure: ternary mixture $\alpha^{\prime}$; Right figure: ternary mixture $\alpha^{\prime \prime}$; Lower figure: ternary mixture at the col point. Open symbols denote run 1 and full symbols run 2.

tribution, represented by the Debye-term, did not impose a significant influence on this behavior. In the range of $\varepsilon \geq 0.04$ the values of $S_{B F}$ are constant in a large reduced temperature range and displayed some kind of "background behavior", $S_{B F}^{b g}$, Fig.(5.53). In the critical mixture $\alpha^{\prime} S_{B F}^{b g} \simeq 0.85 \cdot 10^{-5} \mathrm{~s}^{0.94} \mathrm{~m}^{-1}$, at the col point $S_{B F}^{b g} \simeq 1.39 \cdot 10^{-5} \mathrm{~s}^{0.94} \mathrm{~m}^{-1}$ and for $\alpha^{\prime \prime} S_{B F}^{b g} \simeq 1.20 \cdot 10^{-5} \mathrm{~s}^{0.94} \mathrm{~m}^{-1}$. In comparison, with the binary critical mixture (NE-CH), $S_{B F}^{b g} \simeq 0.8 \cdot 10^{-5} \mathrm{~s}^{0.94} \mathrm{~m}^{-1}$ and with the system (NE-3MP) the largest critical amplitude, $S_{B F}^{b g} \simeq 3.02 \cdot 10^{-5} \mathrm{~s}^{0.94} \mathrm{~m}^{-1}$ emerges. As mentioned before, the behavior of the sound attenuation corresponds with the shape of the fluctuation correlation length versus the weight fraction of (3MP). A question is whether there is some corresponding relation 

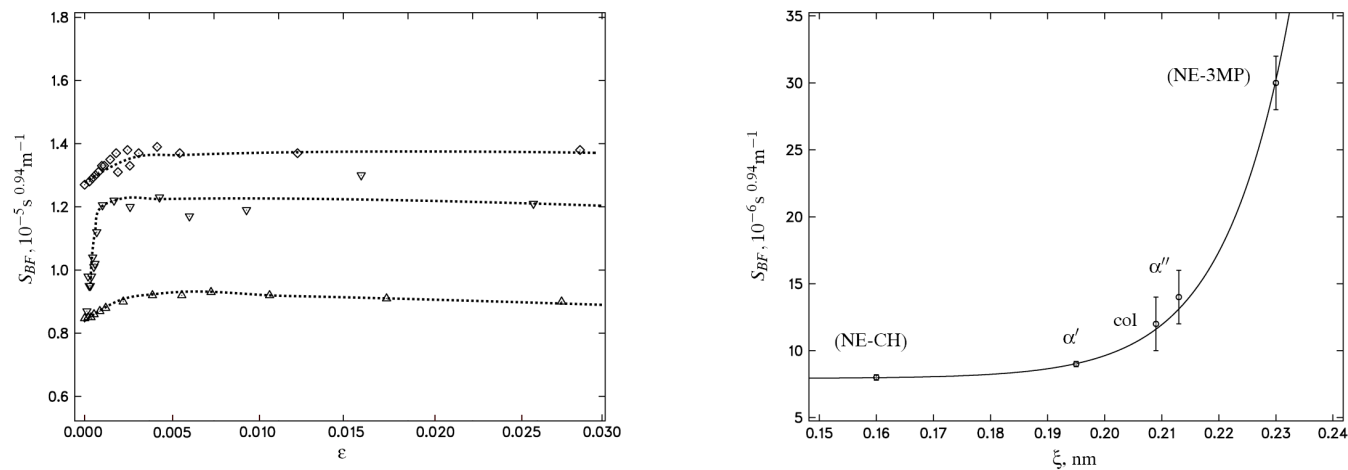

Figure 5.53: Left Figure: Plot of critical amplitudes $S_{B F}$ of three ternary mixtures $\alpha^{\prime}, \Delta$ col point $\diamond$ and $\alpha^{\prime \prime}, \nabla$ versus reduced temperature $\varepsilon$; Right figure: Plot of the critical amplitude $S_{B F}$ versus the fluctuation correlation length, the exponential function line is drawn to guide the eyes.

\begin{tabular}{ccccccccc}
\hline \hline $\begin{array}{c}\text { weight fraction } \\
\text { of (3MP) }\end{array}$ & $\begin{array}{c}\rho, \\
\mathrm{kg} \cdot \mathrm{m}^{-3}\end{array}$ & $\begin{array}{c}c_{s}, \\
\mathrm{~m} \cdot \mathrm{s}^{-1}\end{array}$ & $\begin{array}{c}C_{p b}, \mathrm{~g}^{-1} \cdot \mathrm{K}^{-1} \\
\mathrm{~J} \cdot \mathrm{g}^{-1} \cdot \mathrm{K}^{-1}\end{array}$ & $\begin{array}{c}S_{B F}^{c} \cdot 10^{-5}, \\
\mathrm{~s}^{0.94} \mathrm{~m}^{-1}\end{array}$ & $S_{B F}^{b g} \cdot 10^{-5},|g|_{b g}$ & $|g|_{c}$ \\
\hline 0.000 & 864.5 & 1238 & 1.77 & 6.24 & 0.57 & 0.80 & 0.10 & 0.07 \\
0.082 & 869.8 & 1240 & 1.81 & 3.33 & 0.82 & 0.85 & 0.12 & 0.11 \\
0.200 & 840.4 & 1215 & 1.82 & 2.55 & 1.25 & 1.39 & 0.17 & 0.16 \\
0.300 & 821.2 & 1187 & 1.90 & 2.70 & 0.85 & 1.20 & 0.16 & 0.13 \\
0.534 & 791.2 & 1098 & 1.99 & 2.03 & 2.90 & 2.90 & 0.26 & 0.26 \\
\hline \hline
\end{tabular}

Table 5.8: Densities $\rho$, sound velocities $c_{s}$, background $C_{p b}$ and critical $C_{p c}$ heat capacities at constant pressure, as well as background $S_{B F}^{b g}$ and $S_{B F}^{c}$ critical amplitudes and background $g_{b g}$ and critical $g_{c}$ coupling constants in dependence on the weight fraction of (3MP): background part $C_{p b}$ has been calculated with the aid of Eq.(5.27).

between the critical amplitude $S_{B F}^{b g}$ and the fluctuation correlation length, too. In fact, the plot in Fig.(5.53) demonstrates an interesting dependence of the critical amplitude and the fluctuation correlation length in the case of both binary systems and the corresponding ternary mixtures. This is a usual behavior, when considering the two-scale factor universality relation, which relates the fluctuation correlation length $\xi_{0}$ to the critical heat capacity $C_{p c}$ amplitude. The critical heat capacity amplitude is a substantial quantity in the expression for the critical amplitude Eq.(4.17). Unfortunately, no heat-capacity data are available for the critical ternary mixtures. However, the amplitude of the singular part of heat capacity can be derived from the amplitude of the fluctuation correlation length $\xi_{0}$ using the two-scale factor universality relation. Moreover, the background part $C_{p b}$ of the heat capacity of the ternary mixture, within an error less then $5 \%$, can be approximated assuming an ideal mixing behavior: 

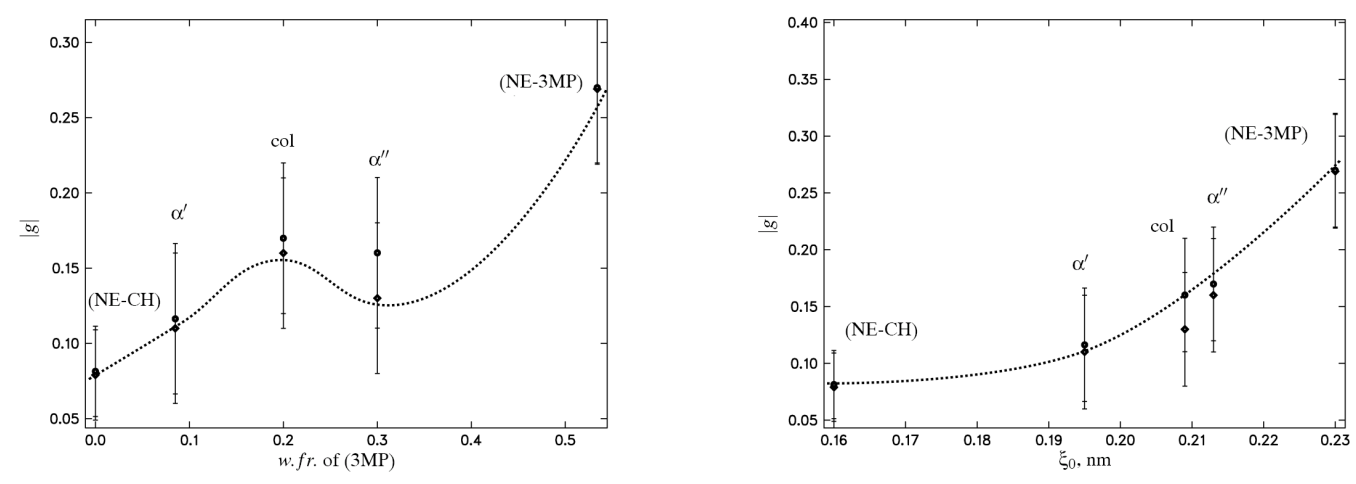

Figure 5.54: Left Figure: Plot of coupling constant $|g|$ versus weight fraction of (3MP); Right figure: Plot of coupling constant $|g|$ versus fluctuation correlation length $\xi_{0} ; \bullet,|g|$ derived from $S_{B F}^{b g} ;,|g|$ derived from $S_{B F}^{c}$.

$$
C_{p b}^{N E-3 M P-C H}=C_{p b}^{N E} x_{c}^{N E}+C_{p b}^{C H} x_{c}^{C H}+C_{p b}^{3 M P} x_{c}^{3 M P}
$$

where $x_{c}^{n}(n=N E, 3 M P, C H)$ is the mole fraction of each component and $C_{p b}$ denotes the background part of the heat capacity. Values for the pure components have been taken from literature [141]. Because of the temperature dependence in the critical amplitude in the ultrasonic spectra, two different critical amplitudes have been used in order to verify the coupling constant $g$. The critical amplitude at the critical point $S_{B F}^{c}$ and the critical amplitude from the background $S_{B F}^{b g}$ which are nearly identical. The evaluated parameters are presented in Table (5.8). The behavior of the coupling constant follows again the shape of the fluctuation correlation length, Fig.(5.54). In the right plot of Fig.(5.54), the coupling constant is displayed versus $\xi_{0}$. 


\subsection{Summarized parameters and surface tension in critical mixtures}

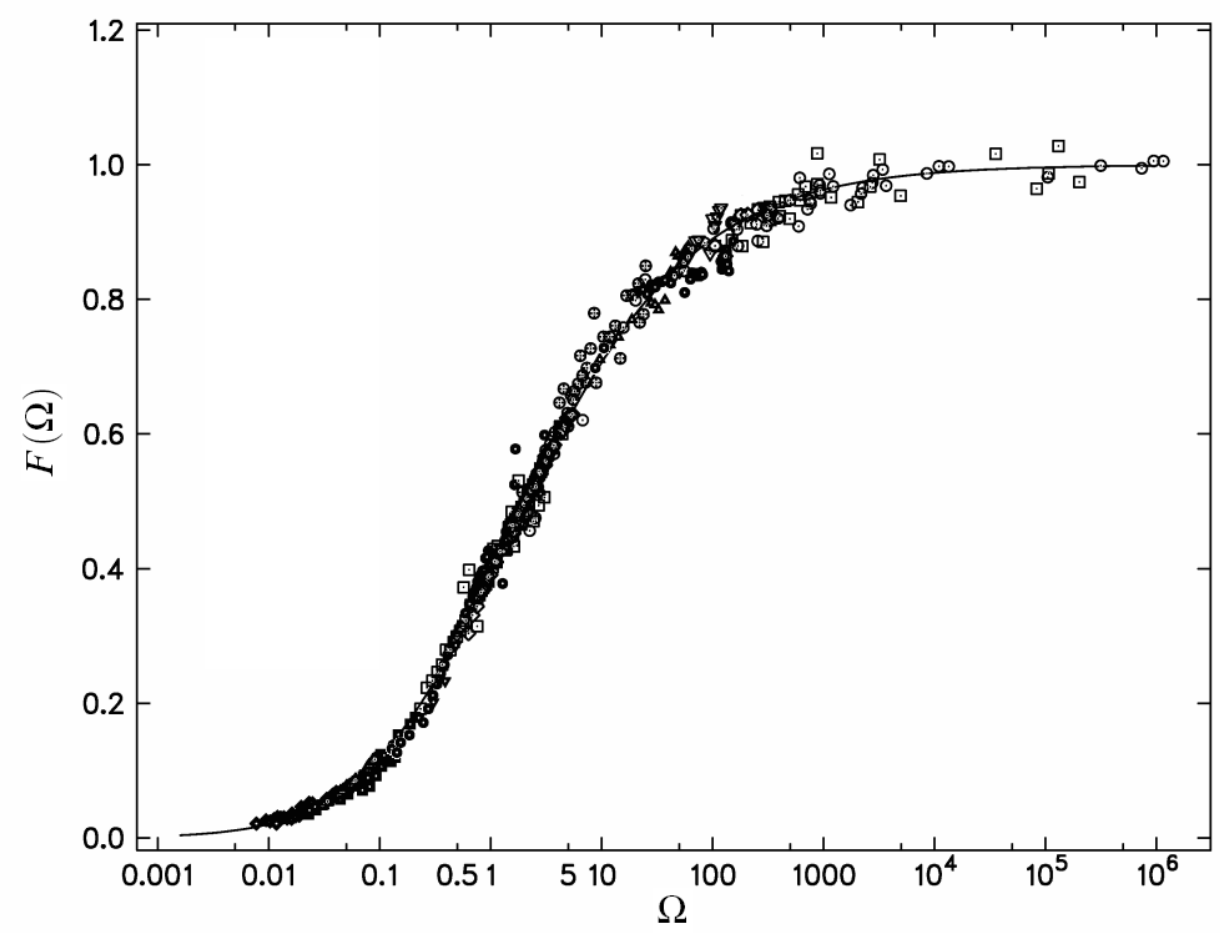

Figure 5.55: Scaling function data of investigated binary and ternary mixtures: $\nabla$ ethanol-dodecane, • methanol-n-hexane, o nitroethane-3-methylpentane, $\square$ nitroethanecyclohexane, $\boldsymbol{\nabla}$ n-pentanol-nitromethane, $\circledast$ isobutoxyethanol-water, $\boldsymbol{\square}$ triethylalmine-water; ternary mixtures $\Delta \alpha^{\prime}$ non-col, $\square$ col, $\diamond \alpha^{\prime \prime}$ non-col.

Parameters which have been obtained from ultrasonic spectrometry, dynamic light scattering and shear viscosity are presented in Table (5.9). It is a fascinating aspect of the dynamic scaling theory of ultrasonic attenuation that, due to scaling of frequency data of different critical mixtures fall on one scaling function, Fig.(5.55). However, the most curious specific system parameter is the characteristic relaxation rate amplitude $\Gamma_{0}$, which according to Bhattacharjee-Ferrell theory, corresponds with the mutual diffusion coefficient $D$ and the fluctuation correlation length $\xi$. In Table (5.9) parameters $\Gamma_{0}$ and $\xi_{0}$ are listed for various binary mixtures with critical demixing point. The isobutoxyethanolwater system exhibits by far the smallest amplitude $\Gamma_{0}$ in the relaxation rate of order parameter fluctuations. In comparison, with the system $n$-pentanol-nitromethane, $\Gamma_{0}$ is 35 


\begin{tabular}{cccccccccc}
\hline \hline critical & $\xi_{0}$ & $A_{\eta}$ & $B_{\eta}$ & $T_{\eta}$ & $q_{c}$ & $q_{D}$ & $\Gamma_{0}$ & $S_{B F}$ & $|g|$ \\
mixture & $\mathrm{nm}$ & $10^{-6}, \mathrm{~Pa} \cdot \mathrm{s}$ & $\mathrm{K}$ & $\mathrm{K}$ & $10^{9} \mathrm{~m}^{-1}$ & $10^{9} \mathrm{~m}^{-1}$ & $10^{9} \mathrm{~s}^{-1}$ & $10^{-5}, \mathrm{~s}^{0.94} \mathrm{~m}^{-1}$ & \\
& \pm 0.02 & \pm 0.02 & \pm 200 & \pm 20 & \pm 0.04 & \pm 0.04 & \pm 2 & $\pm 15 \%$ & \pm 0.02 \\
\hline$(n$-PE-NM) & 0.145 & 0.21 & 2558 & 0 & 187 & 0.39 & 187 & 1.96 & 0.106 \\
$(\mathrm{NE}-\mathrm{CH})$ & 0.160 & 0.55 & 2537 & -57 & $\geq 1000$ & 0.55 & 156 & $7.0^{*}$ & $0.09^{*}$ \\
$(\mathrm{NE}-3 \mathrm{MP})$ & 0.230 & 0.23 & 2877 & -84 & 1.3 & 0.32 & 125 & 2.9 & 0.29 \\
$(\mathrm{ME}-\mathrm{HEX})^{D}$ & 0.330 & 0.29 & 1500 & 0.19 & 1000 & 0.21 & 44 & 0.09 & 0.11 \\
$(\mathrm{EH}-\mathrm{DOD})^{D}$ & 0.370 & 3.80 & 1728 & 0 & 2.4 & 0.86 & 6.4 & 0.07 & 0.10 \\
\hline$\left(\mathrm{TEA}-\mathrm{H}_{2} \mathrm{O}\right)$ & 0.107 & 0.10 & 4394 & 0 & 60 & 0.9 & 96 & 160 & 0.7 \\
$\left(2,6 \mathrm{DMP}-\mathrm{H}_{2} \mathrm{O}\right)$ & 0.198 & 0.14 & 2916 & 0.20 & 1000 & 10 & 25 & 3.97 & 0.17 \\
$\left(i-\mathrm{C}_{4} \mathrm{E}_{1} / \mathrm{H}_{2} \mathrm{O}\right)$ & 0.32 & 0.16 & $255[117]$ & 0 & & & 5.3 & $0.6^{*}$ & 1.77 \\
\hline$\left(\alpha^{\prime}\right)$ & 0.195 & 0.59 & 2958 & -134 & 4.40 & 0.80 & 124 & $0.85^{*}$ & 0.12 \\
$\mathrm{col}^{\prime \prime}$ & 0.213 & 0.61 & 3174 & -174 & 14.40 & 0.60 & 102 & $1.39^{*}$ & 0.17 \\
$\left(\alpha^{\prime \prime}\right)$ & 0.209 & 0.50 & 2818 & -111 & 12.89 & 0.52 & 117 & $1.20^{*}$ & 0.16 \\
\hline \hline
\end{tabular}

Table 5.9: Parameters of the shear viscosity, diffusion coefficient and ultrasonic spectra of critical mixtures without and with one additional noncritical contribution and ternary mixtures: exponent $D$ denotes systems with one Debye process; $*$ stands for dependence on temperature (in such cases $S_{B F}^{b g}$ is presented).

times larger. Assuming, that the life time of fluctuations $\tau_{\xi}=\Gamma_{0}^{-1}$, as inverse characteristic relaxation rate reflects intermolecular properties as well the geometry of considered components the strong variation of $\Gamma_{0}$ of various liquids can be understood. In addition, due to the Coulombic interactions, relaxation from a local nonequilibrium distribution of electrical charges into thermal equilibrium will involve extensive redistribution of ions in ionic solutions and may, therefore, proceed with a smaller relaxation rate than a molecular liquid mixture at the same reduced temperature. A quantity, which may be taken to summarize the above mentioned molecular properties, is the surface tension $\sigma$. If considering critical fluctuations, reflected by the fluctuation relaxation rate $\Gamma_{0}$, to depend on the surface tension, a correlation between both quantities should exist. Based on this idea, Khabibullaev and Mirzaev found a correlation between sound attenuation and surface tension [148], [147]. One of the most important parameters in the determination of the characteristic relaxation rate is the viscosity. The first relation between surface tension and viscosity, has been presented by Pelofsky [149] as a relation between these two thermophysical properties:

$$
\ln \sigma=\ln \mathcal{A}+\frac{\mathcal{B}}{\eta_{s}}
$$

where $\mathcal{A}$ and $\mathcal{B}$ are constants, $\sigma$ is the surface tension, and $\eta_{s}$ the viscosity. According to Eq.(5.28), this empirical expression can be applied for pure and mixed components. Several fluids were shown to follow these relations: $n$-alkanes, benzene, toluene, xylenes, phenol and other aromatics, $n$-alcohols in the range, water and some aqueous solutions. 


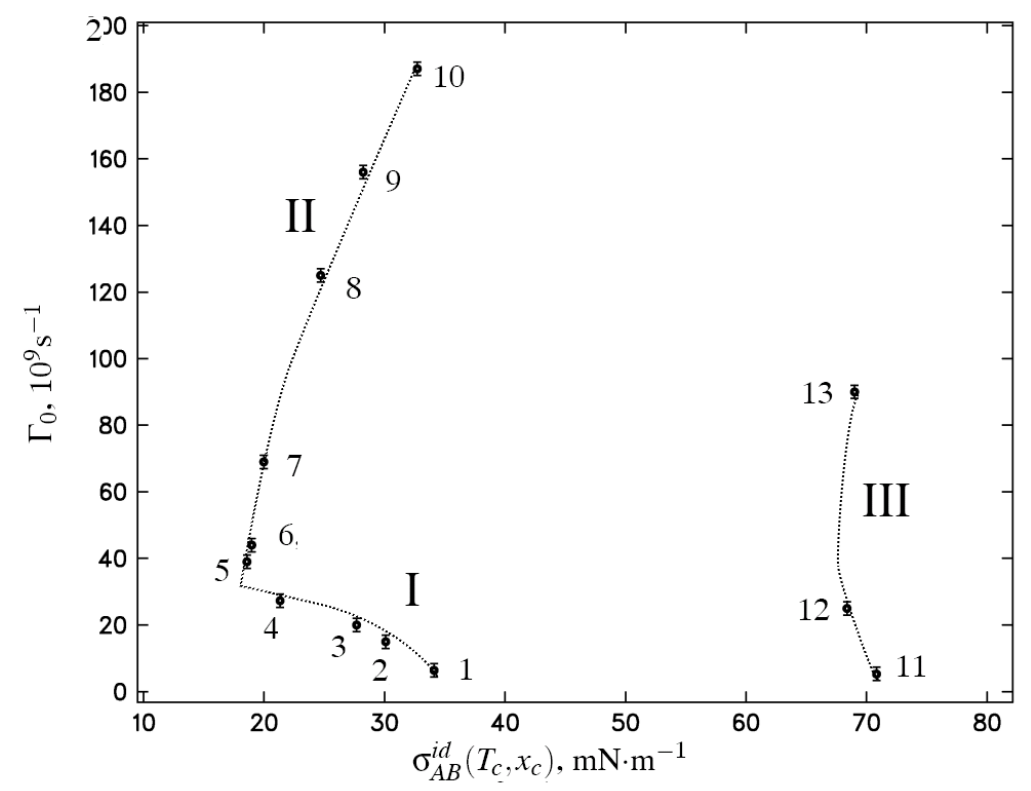

Figure 5.56: Characteristic relaxation rate $\Gamma_{0}$ versus ideal surface tension according to Eq.(5.31): (1) ethanol-dodecane, (2) nitrobenzene-isooctane, (3) nitrobenzene-nhexane, (4) methanol-cyclohexane, (5) benzonitrile-isooctane, (6) methanol-n-hexane, (7) methanol-n-heptane, (8) nitroethane-3-methylpentane, (9) nitroethane-cyclohexane, (10) npentanol-nitromethane, (11) isobutoxyethanol-water, (12) 2,6-dimethylpyridine-water, (13) triethylalmine-water.

Finally, the Eq.(5.28), gives an additional indication for correlations between surface tension and mutual diffusion coefficient, and consequently between mutual diffusion and the fluctuations correlation time. However, it has long been known from experiment that $\partial \sigma / \partial x=0$ ( $x$ denotes composition) at the critical point [150] in binary mixtures. Theory predicts more specifically that, as the critical point is approached, the surface tension $\sigma$ as function of reduced temperature $\varepsilon$, vanishes according to power law with an critical exponent $\mu$ :

$$
\sigma(\varepsilon)=\sigma_{0} \varepsilon^{\mu}
$$

The critical exponent $\mu$, is related to the correlation length exponent $\tilde{v}$, by the Widom [12] scaling law:

$$
\mu=(d-1) \tilde{v}
$$


in which $d$ is the spacial dimension of the system. Unfortunately, it is not possible to find for all considered systems experimental literature data of $\sigma_{0}$, probably, due to experimental problems in the determination of this quantity. Nevertheless, in [151], an expression for an ideal surface tension for a mixture $\sigma_{A B}^{i d}$ has been presented as mole fraction weighted quantity:

$$
\sigma_{A B}^{i d}=x \sigma_{A}+(1-x) \sigma_{B}
$$

here $A, B$ denote the components and $x$ the mole fraction $\left(x=x_{c}\right)$ at critical composition. Surface tension data for pure liquids of all components of binary mixtures presented in Table (5.10) have been found in the literature [151], where for every component the surface tension of four temperatures is listed. Values at temperatures between the tabulated ones could be obtained by linear interpolation with an error of less than $\pm 0.02 \mathrm{mN} / \mathrm{m}$. Taken into account the critical temperature of a mixtures as well as using the Eq.(5.31) the surface tension $\sigma_{A B}^{i d}$ has been calculated, Table (5.10). In order to perform qualitative

\begin{tabular}{|c|c|c|c|c|c|}
\hline $\begin{array}{l}\text { critical } \\
\text { mixture }\end{array}$ & $\begin{array}{c}\Gamma_{0} \\
10^{9} \mathrm{~s}^{-1} \\
\pm 2\end{array}$ & $\begin{array}{c}\xi_{0} \\
\mathrm{~nm} \\
\pm 0.02\end{array}$ & $\begin{array}{c}\sigma_{A}\left(T_{c}\right) \\
\mathrm{mN} \cdot \mathrm{m}^{-1} \\
\pm 0.1\end{array}$ & $\begin{array}{c}\sigma_{B}\left(T_{c}\right) \\
\mathrm{mN} \cdot \mathrm{m}^{-1} \\
\pm 0.1\end{array}$ & $\begin{array}{c}\sigma_{A B}^{i d}\left(T_{c}, x_{c}\right) \\
\mathrm{mN} \cdot \mathrm{m}^{-1} \\
\quad \pm 0.1\end{array}$ \\
\hline isobutoxyethanol-water [77] & 5.3 & 0.320 & 27.69 & 71.66 & 70.82 \\
\hline ethanol-dodecane [99] & 6.4 & 0.370 & 38.62 & 24.52 & 34.11 \\
\hline nitrobenzene-isooctane [142] & 16 & 0.300 & 42.86 & 20.66 & 30.09 \\
\hline nitrobenzene- $n$-hexane [143], [144], [145] & 20 & 0.350 & 42.28 & 16.68 & 27.69 \\
\hline 2,6-dimethylpyridine-water [123] & 25 & 0.198 & 35.44 & 70.47 & 68.37 \\
\hline methanol-cyclohexane [70] & 27 & 0.330 & 20.47 & 22.20 & 21.34 \\
\hline benzonitrile-isooctane [146] & 39 & 0.270 & 21.61 & 21.82 & 18.58 \\
\hline methanol- $n$-heptane [147] & 44 & 0.260 & 21.30 & 16.68 & 18.99 \\
\hline methanol- $n$-hexane [93] & 69 & 0.350 & 21.30 & 18.67 & 19.98 \\
\hline triethylamine-water [136] & 90 & 0.107 & 20.89 & 73.01 & 69.00 \\
\hline nitroethane-3-methylpentane [86] & 125 & 0.230 & 31.94 & 17.45 & 24.70 \\
\hline nitroethane-cyclohexane [90] & 156 & 0.162 & 32.34 & 24.85 & 28.23 \\
\hline n-pentanol-nitromethane [76] & 187 & 0.145 & 25.12 & 37.48 & 32.72 \\
\hline
\end{tabular}

Table 5.10: Amplitude of the characteristic relaxation rate $\Gamma_{0}$, and of the fluctuation correlation length $\xi_{0}$, surface tension $\sigma_{A}\left(T_{c}\right)$, and $\sigma_{B}\left(T_{c}\right)$ of components $\mathbf{A}, \mathbf{B}$ and ideal surface tension $\sigma_{A B}^{i d}\left(T_{c}, x_{c}\right)$ of the mixture, according to Eq.(5.31).

studies of a correlation between characteristic relaxation rate and the surface tension, both quantities have been plotted against another in Fig.(5.56). Unfortunately, the data points do not represent any analytical behavior. Nevertheless, the plot displays three groups of binary critical systems. Group (I) represents binary mixtures, where the characteristic 


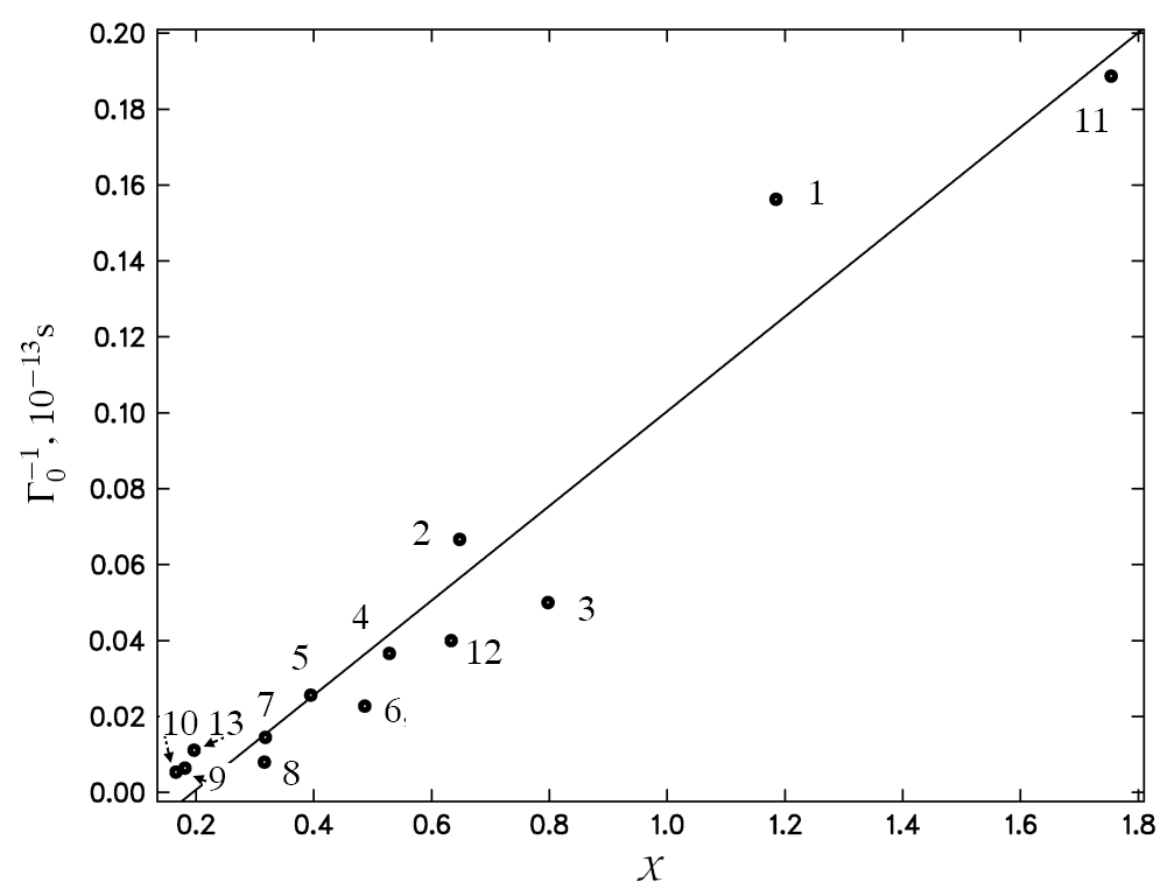

Figure 5.57: Amplitude of the life time of concentration fluctuations in dependence of $x$, according to Eq.(5.32): (1) ethanol-dodecane, (2) nitrobenzene-isooctane, (3) nitrobenzenen-hexane, (4) methanol-cyclohexane, (5) benzonitrile-isooctane, (6) methanol-n-hexane, (7) methanol-n-heptane, (8) nitroethane-3-methylpentane, (9) nitroethane-cyclohexane, (10) npentanol-nitromethane, (11) isobutoxyethanol-water, (12) 2,6-dimethylpyridine-water, (13) triethylamine-water.

relaxation rate decreases with increasing surface tension, while group (II) displays an opposite trend. The third group (III) shows the behavior of binary aqueous solutions, with by far the largest surface tension. However, this kind of qualitative studies give an indication that there must exists an additional parameter which interacts additionally with the surface tension. In fact it has been argued by Fisk and Widom and recognized later as an aspect of the tow-scale factor universality that there exists a universal combination of critical amplitudes involving the surface tension amplitude $\sigma_{0}$, [153]. According to Brézin [154], the simplest way to define the universal combination is to notice that the free interfacial energy per unit area (divided by $k_{B} T$ ), multiplied by an area as defined by the correlation length, is both temperature independent and universal in the vicinity of $T_{c}$. Owing to this argument, the following expression can be written, [30]:

$$
\chi=\frac{\sigma(\varepsilon) \xi(\varepsilon)^{2}}{k_{B} T_{c}},
$$


where $X=1 / 4 \cdot \pi k$ and $k$ is a dimensionless quantity between 1.39 and 1.57 from theory, $k_{B}$, is Boltzmann's constant and $\varepsilon$ is the reduced temperature. However, it was also reported in [154], that $k$ lies in the interesting range between 0.5 and 2.00. The Eq.(5.32) plays a key role in understanding the nature of critical wetting and it is likewise a powerful expression, which connects the surface tension and fluctuation correlation length. As mentioned before, no data of surface tension according to Eq.(5.29) are available in the literature the quantitatively analyze Eq.(5.32). However, using the same surface tension values, calculated with the aid of Eq.(5.31), the dependence of the characteristic relaxation rate $\Gamma_{0}$, and accordingly the life time of fluctuations could be studied as a function of the correlation length as well as surface tension. In fact the qualitative plot of life time of fluctuations $\Gamma_{0}^{-1}$ demonstrates a linear dependence on quantity $X$ Fig.(5.57). Once more it should be underlined, that this are only qualitative studies. 



\section{Conclusions and Outlook}

In this thesis dynamic light scattering and shear viscosity measurements have been performed and broad band ultrasonic spectrometry was carried out in the frequency range between $180 \mathrm{kHz}$ and $500 \mathrm{MHz}$. Evaluating the experimental data for liquid mixtures of critical composition particular attention has been given to the applicability of BhattacharjeeFerrell dynamic scaling model and to corrections for the effects from the crossover from Ising to mean-field behavior. Three types of critical liquids have been considered: binary mixtures without complex background contributions in their ultrasonic spectra $(n-$ pentanol-nitromethane, nitroethane-cyclohexane, nitroethane-3-methylpentane, methanol-hexane, and ethanol-dodecane), binary mixtures with additional relaxations in the time domain of critical fluctuations (2,6-dimethylpyridin-water, isobutoxyethanol-water, triethylamine-water), and ternary mixtures with concentrations selected along the plait-point line (nitroethane-3-methylpentane-cyclohexane). With the latter system interest has been particulary directed to the dependence of critical parameters up on the concentration of a constituent.

In the homogenous region near a consolute point the critical dynamics of binary liquid mixtures without additional sonic relaxations can be consistently represented by the Bhattacharjee-Ferrell theory. The scaling function of the more recent Onuki and FolkMoser theories exhibit systematic deviations from the experimental data. Using the former theory the scaled half-attenuation frequency $\Omega_{1 / 2}$ nicely agrees with the value 2.1 as predicted by Bhattacharjee and Ferrell. An exception is the system $n$-pentanol-nitromethane for which the slightly smaller $\Omega_{1 / 2}=1.86$ is found. A noteworthy result is the finding that relaxation rate data from dynamic light scattering and shear viscosity measurements can be used in the theoretical description of the ultrasonic spectra.

In the case of the critical binary mixture nitroethane-cyclohexane an anomalous variation in the amplitude of the critical contribution $S_{B F}$ has been found. This temperature dependence is obviously due to a temperature dependence in the adiabatic coupling constant $g$ which results from the predominance of the $T$ dependent thermal expansion coefficient. If this effect is disregarded scaling function data from different temperature runs do not condense onto one curve. In the critical binary liquids ethanol-dodecane as well as methanol-hexane a relaxation process, with relaxation time in the nanosecond range $\tau_{D}=19.1 \mathrm{~ns}$, and $\tau_{D}=2 \mathrm{~ns}$, respectively, has been found. It was assigned to a reaction in 
which $n$ alcohol molecules associate to $n$-mers. Assuming this relaxation to contribute its low frequency wing to the spectra, where special temperature dependent measurements for the ethanol-dodecane and methanol-hexane mixture of critical composition were performed, leads to an excellent agreement of the experimental scaling function with the theoretical form of the Bhattacharjee-Ferrell model. This is a noteworthy result because the evaluation of experimental data needs only one adjustable parameter.

Use of the Bhattacharjee-Ferrell model for the analytical representation of the critical part in the ultrasonic attenuation spectra of more complicated systems allows for a favorable description of further relaxation terms. With the assumption that the contributions from chemical relaxations contribute additively to the critical contributions the ultrasonic spectra as well as the scaling function of the critical systems can be well represented in terms of the Bhattacharjee-Ferrell model. This was shown for the binary mixtures isobutoxyethanol-water and 2,6-dimethylpyridin-water. The system triethylamine-water, which includes two Debye-type relaxation terms, reflecting noncritical chemical reactions in addition to the high-frequency part, indicates a coupling between the critical dynamics and the noncritical relaxation terms. The experimental scaling function agrees with the Bhattacharjee-Ferrell function only, when both Debye relaxation terms are allowed slow down near the critical point. This is also true for the term that is assigned to a monomolecular structural isomerization of TEA which is expected to be independent from fluctuations. This finding may be taken to indicate water to play a noticeable role in the essentially uni-molecular isomerization. Water probably participates in the relaxation due to an extensive rearrangement of hydration shells associated with the isomerization of TEA. Unfortunately, it is difficult to study the behavior of the Debye-type relaxations without interferences by the critical term in the attenuation spectra. However, the slowing of critical relaxations near the critical temperature, has been also reported by Procaccia, Krichevskii and Wheeler.

The results for ternary mixtures demonstrate, that the ternary $\alpha^{\prime}$, col-point and $\alpha^{\prime \prime}$ systems (nitroethane-3-methylpentane-cyclohexane ) can be also well represented by the empirical Bhattacharjee-Ferrell scaling function with scaled half attenuation frequency $\Omega_{1 / 2}=$ 2.1. For most quantities, such as the characteristic relaxation rate amplitude $\Gamma_{0}$, the amplitude of the fluctuation correlation length $\xi_{0}$, as well as the background parameters $A_{\eta}$, $B_{\eta}$, and $T_{\eta}$ of the shear viscosity, an evident dependence on the weight fraction of the third component (3-methylpentane) results. Especially, studies of the crossover behavior in dependence of the distance of the reduced temperature from the critical temperature as well from the used model of background viscosity indicate that only by viscosity measurements in a wide temperature range the correct determination of the fluctuation correlation length amplitude $\xi_{0}$ and the cut-off wave numbers $q_{c}, q_{D}$ (and consequently $\Gamma_{0}$ ) is guaranteed. Furthermore, the results of the low frequency sound attenuation measurements 
do not confirm the conclusion by Mistura that close to the col point the critical absorption should decrease with increasing number of components. In the case of the ternary systems of the present thesis this quantities corresponds rather with the fluctuation correlation length from dynamic light scattering. The same correspondence can be found with the critical amplitude $S_{B F}$, which seems to demonstrate the same anomaly as the well known binary system nitroethane-cyclohexane.

Additionally, in order to more closely consider the large variation of characteristic relaxation rate amplitude $\Gamma_{0}$ of various binary systems, its dependence on surface tension of that quantity has been considered. Unfortunately, there are only insufficient surface tension data at the critical point. Therefore only a qualitative analysis of such correlation could be done. This analysis revealed a dependence of $\Gamma_{0}$ on the surface tension.

In summary, the results clearly demonstrate that the Bhattacharjee-Ferrell theory and the crossover theory nicely represent the experimental ultrasonic attenuation data as well as the shear viscosity and dynamic light scattering data. This is true for binary and also for ternary mixtures. Experimental critical exponents agree with those from theory. There is, however, a necessity for the development of new theories treating the coupling between chemical relaxations and critical fluctuations. Furthermore, more investigations on ternary mixtures with the aid of broad-band ultrasonic are necessary to show that the dynamic scaling theory of Bhattacharjee-Ferrell applies also to those systems. 


\section{Bibliography}

[1] Brady P. R., Choptuik P. W., Gundlach C., Neilsen D. W.; Class. Quant. Grav. 19 (2002) 6359

[2] Gundlach C.; Living Reviews in Relativity (1999)

[3] Scherfeld D., Kahya N., Schwilley P.; Biophys. J. 85 (2003) 3758

[4] Veatch S. L., Keller S. L. ; Biophys. J. 85 (2003) 3074

[5] Veatch S. L., Keller S. L. ; Phys. Rev. Lett. (2005) 148101

[6] Ising E. Z.; Phys. 31 (1925) 253

[7] Landau L. D., Lifschitz J. M.; Lehrbuch der theoretischen Physik (Band 5) Statistische Physik Teil 1, Auflage: 8. Akademie Verlag (1991)

[8] Onsager L.; Phys. Rev. 65 (1944) 117

[9] Fisher M. E.; Phys. Rev. 176 (1968) 257

[10] Fisher M. E., Scesney P. E.; Phys. Rev. A 2 (1970) 825

[11] Fisher M. E.; Rev. Mod. Phys. 46 (1974) 597

[12] Widom B.; J. Chem. Phys. 43 (1965) 3898

[13] Patashinskii A. Z., Pokrovskii V. L.; Sov. Phys. JETP 23 (1966) 293

[14] Kadanoff L. P.; Physics (Long Island City, N.Y.) 2 (1966) 263

[15] Wilson K. G.; Phys. Rev. B 4 (1971) 3174

[16] Burstyn H. C., Sengers J. V., Bhattacharjee J. K., Ferrell R. A.; Phys. Rev. A 28 (1983) 1567

[17] Bhattacharjee J. K., Ferrell R. A., Basu R. S., Sengers J. V.; Phys. Rev. A 24 (1981) 1469 
[18] Ferrell R. A., Bhattacharjee J. K.; Phys. Lett. A 86 (1981) 109

[19] Bhattacharjee J. K., Ferrell R. A.; Phys. Lett. A 88 (1982) 77

[20] Ferrell R. A., Bhattacharjee J. K.; Phys. Rev. A 31 (1985) 1788

[21] Bhattacharjee J. K., Ferrell R. A.; Physica A 250 (1998) 83

[22] Ferrell R. A.; Phys. Lett. 24 (1970) 1169

[23] Folk R., Moser G.; Phys. Rev. E 57 (1998) 705

[24] Folk R., Moser G.; Phys. Rev. E 58 (1998) 6246

[25] Onuki A.; J. Phys. Soc. Jpn. 66 (1997) 511

[26] Onuki A.; Phys. Rev. E 55 (1997) 403

[27] Procaccia I., Gitterman M.; Phys. Rev. A 27 (1983) 555

[28] Griffiths R. B., Wheeler J. C.; Phys. Rev. A 2 (1970) 1047

[29] Ornstein L. S., Zernike F.; Proc. Acad. Sci. (Amsterdam) 17 (1914) 1452

[30] Anisimov M. A.; Critical Phenomena in Liquids and Liquid Crystals Gordon and Breach London (1987)

[31] Cerdeiriña C. A., Anisimov M. A., Sengers J. V.; Chem. Phys. Lett. 424 (2006) 414

[32] Ferrell R. A., Menyhárd N., Schmidt H., Schwabl F., Szépfalusy P.; Phys. Rev. Lett. 18 (1967) 891

[33] Halperin B. I., Hohenberg P. C.; Phys. Rev. 177 (1969) 952

[34] Hohenberg P. C., Halperin B. I.; Rev. Mod. Phys. 49 (1977) 435

[35] Gillou J. C., Zinn-Justin J.; Phys. Rev. Lett. 39 (1977) 95

[36] Burstyn H. C., Sengers J. V.; Phys. Rev. A 25 (1998) 448

[37] Bak C., Goldburg W.; Phys. Rev. Lett. 2 (1969) 1218

[38] Goldburg W., Bak C., Pusey P.; Phys. Rev. Lett. 25 (1970) 1430

[39] Hao H., Ferrell R. A., Bhattacharjee J. K.; Phys. Rev. E 71 (2005) 021201

[40] Müller O.; Dissertation, Mat.-Nat.-Tech. Univ. Halle-Wittenberg (2001) 
[41] Müller O., Winkellmann J.; Phys. Rev. E 60 (1999) 4453

[42] Smoluchowski M.; Ann. Phys. (Leipzig) 25 (1908) 205

[43] Einstein A.; Ann. Phys. (Leipzig) 33 (1910) 1275

[44] Sadus R. J.; High Pressure Phase Behaviour of Multicomponent Fluid Mixtures, Elsevier, Amsterdam (1992)

[45] Francis A. W.; J. Am. Chem. Soc. 76 (1954) 383

[46] Chu B.; Laser Light Scattering - Basic Principles and Practice - Second Edition,; Academic Press, Inc. (1991)

[47] Berne B. J., Pecora R.; Dynamic Light Scattering with Applications to Chemistry, Biology and Physics, Malabar, (1990)

[48] Langevin D.; Light Scattering by Liquid Surfaces and Complementary Techniques, New York, (1992)

[49] Stanley H. E.; Introduction to Phase Transitions and Critical Phenomena, Oxford University Press, (1987)

[50] Stokes G.; Trans. Cambridge Philos. Soc. 8 (1845) 287

[51] Einstein A.; Ann. Phys. (Leipzig) 17 (1905) 549

[52] Bhatia A. B.; Ultrasonic Absorption, Oxford University Press (1967)

[53] Kirchhoff G.; Pogg. Ann. Physik 134 (1868) 177

[54] Behrends R., Kaatze U.; J. Mol. Liq. (2003) 107

[55] Strehlow H.; Rapid Reactions in Solutions, VCH Verlagsgesellschaft, Weinheim (1992)

[56] Hill R. M.; Nature 275 (1978) 96

[57] Hill R. M.; Phys. Stat. Sol. 103 (1981) 319

[58] Menzel K., Rupprecht A., Kaatze U.; J. Acoust. Soc. Am. 104 (1998) 2741

[59] Lamb J.; Physical Acoustics - Vol.II, Part A, New York (1965)

[60] Eggers F. and Kaatze U.; Meas. Sci. Technol. 7 (1996) 1

[61] Labhardt A.; Dissertation, Phil.-Nat.-Fak. Univ. Basel (1975) 
[62] Labhardt A., Schwarz G.; Ber. Bunsges. Phys. Chem. 80 (1976) 83

[63] Lautscham K.; Dissertation, Mat.-Nat.-Fak. Univ. Göttingen (1986)

[64] Behrends R.; Kaatze U.; Meas. Sci. Technol. 12 (2001) 519

[65] Herzfeld K. E., Rice F. O.; Phys. Rev. 31 (1928) 77

[66] Kawasaki K.; Ann. Phys. (N.Y) 61 (1970) 1

[67] Behrends R., Kaatze U.; Europhys. Lett 65 (2004) 221

[68] Albright P. C., Sengers J. V., Nicoll J. F., Ley-Koo M.; Int. J. Thermophys. 7 (1986) 75

[69] Matos Lopes M. L. S., Nieto de Castro C. A., Sengers J. V.; Int. J. Thermophys. 13 (1992) 283

[70] Behrends R., Kaatze U., Schach M.; J. Chem. Phys. (2003) 1199192

[71] Wagner M., Stanga O., Schröer W.; Phys. Chem. Chem. Phys. 4 (2002) 5300

[72] Pfeuty P., Tolous G.; Introduction to the Renormalization Group and to Critical Phenomena, Wiley, London, (1977)

[73] Kaatze U., Wehrmann B., Pottel R.; J. Phys. E: Sci. Instrum. 20 (1987) 1015

[74] Cerdeiriña C. A., Troncoso J., Carballo E., Romaní R.; Phys. Rev. E 66 (2002) 031507

[75] Flewelling A. C., DeFonseka R. J., Khaleeli N., Partee J., Jacobs D. T.; J. Chem. Phys. 140 (1996) 8048

[76] Iwanowski I., Behrends R., Kaatze U.; J. Chem. Phys. 120 (2004) 9192

[77] Iwanowski I., Mirzaev S. Z., Kaatze U.; Phys. Rev. E 73 (2006) 061508

[78] Kadanoff L. P., Swift J.; Phys. Rev. 166 (1968) 89

[79] Rebillot P. F., Jacobs D. T.; J. Chem. Phys. 109 (1998) 4009

[80] Bervillier C., Godrèche C.; Phys. Rev. B 21 (1980) 5427

[81] Łabowski M., Hornowski T.; J. Acoust. Soc. Am. 81 (1987) 1421

[82] Hornowski T., Łabowski M.; Acta. Phys. Pol 79 (1991) 671 
[83] Mirzaev S. Z., Kaatze U.; Phys. Rev. E 57 (2002) 021509

[84] Sanchez G., Garland C. W.; J. Chem. Phys. 79 (1983) 3100

[85] Garland C. W., Sanchez G.; J. Chem. Phys. 79 (1983) 3090

[86] Iwanowski I., Leluk K., Rudowski K., Kaatze U.; J. Phys. Chem. A 110 (2006) 4313

[87] Stein A., Allegra J. C., Allen G. F.; J. Chem. Phys. 55 (1971) 4265

[88] Greer S. C., Hocken R.; J. Chem. Phys. 63 (1975) 5067

[89] Mirzaev S. Z., Telgmann T., Kaatze U.; Phys. Rev. E 61 (2000) 542

[90] Behrends R., Iwanowski I., Kosmowska M., Szala A., Kaatze U.; J. Phys. Chem. 121 (2004) 5929

[91] Djavanbakht A., Lang J., Zana R.; J. Phys. Chem. 81 (1977) 2620

[92] Golubovskii N. Yu.; Fiz. Znidk. Sostoyania. Resp. Mezhved. Nauch. Sb. 6 (1978) 34

[93] Iwanowski I., Sattarow A., Behrends R., Mirzaev S.Z., Kaatze U.; J. Phys. Chem. 121 (2006) 144505

[94] Timmermans J., Kohnstamm P.; Proc. Acad. Sci. (Amsterdam) 12 (1909) 234

[95] Mayers D. B., Smith R. A., Katz J., Scott R. L.; J. Phys. Chem. 70 (1966) 3341

[96] Garland C. W., Lai C. N.; J. Chem. Phys. 69 (1978) 1342

[97] Behrends R., Telgmann T., Kaatze U.; J. Chem. Phys. 117 (2002) 9828

[98] Mirzaev S. Z., Kaatze U.; Phys. Rev. E 65 (2002) 021509

[99] Mirzaev S. Z., Iwanowski I., Kaatze U.; Chem. Phys. Lett. 435 (2007) 263

[100] Mirzaev S. Z., Iwanowski I., Kaatze U.; J. Phys. D: Appl. Phys. 40 (2007) 3248

[101] Kaatze U., Mirzaev S. Z.; J. Phys. Chem. 104 (2000) 5430

[102] Degiorgio V., Piazza R., Corti M., Minero C.; J. Chem. Phys. 82 (1985) 1025

[103] Corti M., Degiorgio V.; Phys. Rev. Lett. 55 (1985) 2005

[104] Dietler G., Cannell D. S.; Phys. Rev. Lett. 60 (1988) 1852

[105] Hamano K., Kuwahara N., Mitsushima I., Kubota K., Kamura T.; J. Chem. Phys. 94 (1991) 2172 
[106] Telgmann T., Kaatze U.; J. Phys. Chem. A 104 (2000) 1085

[107] Telgmann T., Kaatze U.; J. Phys. Chem. A 104 (2000) 4846

[108] Telgmann T., Kaatze U.; Langmuir 18 (2002) 3068

[109] Hanke E., Telgmann T., Kaatze U.; Tenside, Surf. Det. 42 (2005) 23

[110] Menzel K., Mirzaev S. Z., Kaatze U.; Phys. Rev. E 68 (2003) 011501

[111] Lesemann M., Martin A., Belkoura L., Woermann D.; Ber. Bunsenges. Phys. Chem. 101 (1997) 228

[112] Höhn F.; Dissertation, Universität zu Köln, Köln (1992)

[113] Telgmann T, Kaatze U.; J. Phys. Chem. B 101 (1997) 7766

[114] Würz W., Grubic M., Woermann D.; Ber. Bunsenges. Phys. Chem. 96 (1992) 1460

[115] Zimmer H. J., Woermann D.; Ber. Bunsenges. Phys. Chem. 95 (1991) 533

[116] Mirzaev S. Z., Behrends R., Heimburg T., Haller J., Kaatze U.; J. Chem. Phys. 124 (2006) 144517

[117] Limberg S., Belkoura L., Woermann D.; J. Mol. Liq. 73-74 (1997) 223

[118] Kaatze U., Woermann D.; J. Phys. Chem 88 (1984) 284

[119] Ito N., Kato T.; J. Phys. Chem. 88 (1984) 801

[120] Kaatze U., Schreiber U.; Chem. Phys. Lett. 148 (1988) 241

[121] Sacconi L., Paoletti P., Ciampolini M.; J. Am. Chem. Soc. 82 (1960) 3831

[122] Behrends R., Kaatze U.; Phys. Rev. E 68 (2003) 011205

[123] Mirzaev S. Z., Iwanowski I., Zaitdinov M., Kaatze U.; Chem. Phys. Lett. 431 (2006) 308

[124] Yun S. S. J. Chem. Phys. 52 (1970) 5200

[125] Garland, C. W.; Lai, C.- N. J. Chem. Phys. 69 (1978) 1342

[126] Haesell, E. L. Proc. R. Soc. London, Ser. A 237 (1957) 1209

[127] Hentschel, H. G. E.; Procaccia, I. J. Chem. Phys. 76 (1982) 666

[128] Kaatze, U.; Mirzaev, S. Z. J. Phys. Chem. A 104 (2000) 5430 
[129] Nagasawa Y., Nakagawa Y., Nagafuji A., Okada T., Miyaska H.; J. Mol. Sruc. 735-736 (2005) 217

[130] Milner S. T., Martin P. C; Phys. Rev. A 33 (1986) 1996

[131] Kenneth R. H., Hanh N. L.; J. Chem. Soc. Faraday Trans. 91 (1995) 4071

[132] Gitterman M.; J. Stat. Phys. (1990) 58707

[133] Wheeler J. C.; Petschek R. G.; Phys. Rev. A 28 (1983) 2442

[134] Krichevskii I.R., Tsekhanskaja Yu. V, Rozhnovskaja L. N.; Russ. J. Phys. Chem. 43 (1969) 1393

[135] Verrall R. E., Nomura H.; J. Sol. Chem 6 (1976) 217

[136] Iwanowski I., Kaatze U.; J. Phys. Chem B 111 (2007) 1438

[137] Zhang L., Wang Q., Liu Y. C., Zhang L. Z.; J. Chem. Phys. 125 (2006) 104502

[138] Davidovich L. A., Izbasarov B., Khabibullaev P. K., Khaliulin M. G.; Akust. Zh. 19 (1971) 453

[139] D’Arrigo G., Tartaglia P.; J. Chem. Phys. 69 (1978) 429

[140] Mistura L.; J. Phys. Chem. 57 (1972) 2311

[141] Speight J. G.; Lange's Handbook of Chemistry 16th edition, The McGraw-Hill Companies (2005)

[142] Jaschull G., Dunker H., Woermann D.; Ber. Bunsenges. Phys. Chem. 88 (1984) 630

[143] Tanaka H., Wada Y., Nakajima H.; Chem. Phys. 75 (1983) 37

[144] Chen S. H., Polonsky N.; Opt. Commun. 1 (1969) 64

[145] Chen S. H., Lai C. C., Rouch J., Tartaglia P.; Phys. Rev. A 27 (1983) 1086

[146] Hornowski T., Łabowski M.; Arch. Acoust. 21 (1996) 53

[147] Mirzaev S. Z.; Dissertation, Heat Physics Department, Uzbekistan Academy of Sciences, Tashkent (1996)

[148] Khabibullaev, Mirzaev S. Z., Saidov A. A., Shinder I. I.; Doklady Akademii Nauk 345 (1995) 756

[149] Pelofsky A. H.; J. Chem. Eng. Data 11 (1966) 394 
[150] Wellm J.; Z. Phys. Chem. Abt. B28 (1935) 119

[151] Dewan R. K., Mehta S. K.; Chemical Monthly Springer (1990) B28 (1935) 119

[152] Jasper J. J.; J. Phys. Chem. Ref. Data 1 (1972) 841

[153] Fisk S., Widom B.; J. Chem. Phys. 50 (1969) 3219

[154] Brézin E., Shechao F.; Phys. Rev. B 29 (1984) 472 


\section{Acknowledgements}

Herrn Prof. Dr. Werner Lauterborn danke ich für die Betreuung dieser Arbeit. Herrn Prof. Dr. Christoph Schmidt danke ich für die Übernahme des Koreferates.

Mein besonderer Dank gilt Herrn Dr. Udo Kaatze, der mich in jeder Situation tatkräftig unterstützt hat und mir zu jedem Zeitpunkt für Fragen offenstand. Seine motivierende Art hat mir bei der Fertigstellung dieser Arbeit sehr geholfen.

Mein Dank gilt auch Herrn Prof. Dr. Sirojiddin Mirzeav, der mich durch zahlreiche konstruktive Diskussionen bei der Verwirklichung dieser Dissertation sehr unterstützt hat.

Bei Herrn Dipl. Phys. Julian Haller möchte ich mich für die unzähligen heiteren Momente und konstruktiven Unterhaltungen, sowohl auf wissenschaftlicher wie auch auf privater Ebene, bedanken.

Dr. Vitaliy Oliynyk danke ich für die wissenschaftliche Unterstützung bei den AFMMessungen und für seine einzigartige und humorvolle Art mit Problemen umzugehen. Bei Dr. Ralph Behrends, Dr. Ralf Hagen und Dr. Rüdiger Polacek bedanke ich mich für ihre offene Art und für das kreieren einer wunderbaren Arbeitsatmosphäre.

Den Labormitarbeitern Kerstin von Roden, Ulrike Schulz, Karola Fritz und David Gottschalk danke ich für die Durchführung ergänzender Messungen.

Allen Mitarbeiterinnen und Mitarbeitern unseres Institutes danke ich für die hilfreiche Zusammenarbeit, sowie die wundervolle Arbeitsatmosphäre. Bei Herrn Dieter Hille und allen Mitarbeitern der Mechanikwerkstatt, sowie bei Herrn Dr. Karl Lautscham und der gesamten Elektronikwerkstatt möchte ich mich für die professionellen und schnellen Reparaturen an den Messgeräten bedanken. Nicht zuletzt danke ich dem Reinigungspersonal, das zu der schönen Arbeitsatmosphäre einen grossen Beitrag geleistet hat.

Der Deutschen Forschungsgemeinschaft danke ich herzlich für die Finanzierung dieser Arbeit. 
Nicht zuletzt, möchte ich mich bei all den Menschen bedanken, die nicht in direkter Verbindung zu der Arbeit stehen, die jedoch eine wichtige Rolle in meinem persönlichen Leben spielen.

Meinem ersten Mathematiklehrer in Deutschland, Herrn Edmund Wilhelm, danke ich für die Unterstützung meiner naturwissenschaftlichen Interessen. Seine Art die Welt der Wissenschaft zu sehen, hatte einen enormen Einfluss auf meine Person.

Ein besonderer Dank gilt meiner Freundin Antje Lücke für ihre Geduld, für ihr Verständnis und für die zuverlässige Versorgung mit Proviant während der Nachtmessungen.

Moim rodzicom Annie i Kazimierzowi Iwanowskim, dziękuję z całego serca za wszystko co dla mnie zrobili. Byli, i są, dla mnie zawsze wielkạ podporą w każdym rodzaju. To wszystko co im zawdziȩczam, nie da się opisać słowami. Dziȩkuję!!!

Ireneusz Iwanowski 


\section{Curriculum vitae}

Ireneusz Iwanowski

Görlitzer Str. 10a

D-37085 Göttingen

\section{Biographische Daten}

Geburtsdatum: $\quad$ 8. September 1977

Geburtsort: $\quad$ Sorau (Żary, Polen)

Staatsangehörigkeit: deutsch

\section{Bildungsgang}

1984-1989 Grundschule in Sorau (Polen), (09/1989 Umzug Deutschland)

09/1989-06/1994 Kooperative Gesamtschule in Witzenhausen

09/1994-06/1997 Otto-Hahn-Gymnasium in Göttingen, Abitur (06/1997)

10/1997 Beginn des Studiums der Physik und Mathematik an der Georg-August-Universität Göttingen

06/1999 Vordiplom in Physik an der Universität Göttingen

04/2002-09/2003 Diplomarbeit, Fakultät für Physik an der Georg-August-Universität in Göttingen betreut durch Dr. Udo Kaatze und Prof. Dr. Dirk Ronneberger Thema: "Verifizierung der dynamischen Skalierungshypothese mittels Ultraschallspektroskopie und quasielastischer Lichtstreuung"

seit 04/2004 Wissenschaftlicher Mitarbeiter am Dritten Physikalischen Institut der Universität Göttingen

seit 04/2004 Promotionsstudium, Fakultät für Physik an der Georg-August-Universität zu Göttingen Referent: Prof. Dr. Werner Lauterborn Thema: "Critical Behavior and Crossover Effects in the Properties of Binary and Ternary Mixtures and Verification of the Dynamic Scaling Conception"

Göttingen, 31.Oktober 2007 